Prepared for the U.S. Department of Energy

under Contract DE-AC05-76RL01830

\title{
Second Line of Defense Master Spares Catalog
}

\author{
DL Henderson \\ G Muller \\ TM Mercier

\begin{abstract}
RT Brigantic
CJ Perkins

SK Cooley
\end{abstract}

November 2012

\section{Pacific Northwest}

NATIONAL LABORATORY

Proudly Operated by Battelle Since 1965 


\title{
DISCLAIMER
}

This report was prepared as an account of work sponsored by an agency of the United States Government. Neither the United States Government nor any agency thereof, nor Battelle Memorial Institute, nor any of their employees, makes any warranty, express or implied, or assumes any legal liability or responsibility for the accuracy, completeness, or usefulness of any information, apparatus, product, or process disclosed, or represents that its use would not infringe privately owned rights. Reference herein to any specific commercial product, process, or service by trade name, trademark, manufacturer, or otherwise does not necessarily constitute or imply its endorsement, recommendation, or favoring by the United States Government or any agency thereof, or Battelle Memorial Institute. The views and opinions of authors expressed herein do not necessarily state or reflect those of the United States Government or any agency thereof.

\author{
PACIFIC NORTHWEST NATIONAL LABORATORY \\ operated by \\ BATTELLE \\ for the \\ UNITED STATES DEPARTMENT OF ENERGY \\ under Contract DE-AC05-76RL01830 \\ Printed in the United States of America \\ Available to DOE and DOE contractors from the \\ Office of Scientific and Technical Information, \\ P.O. Box 62, Oak Ridge, TN 37831-0062; \\ ph: (865) 576-8401 \\ fax: $(865) 576-5728$ \\ email: reports@adonis.osti.gov
}

Available to the public from the National Technical Information Service

5301 Shawnee Rd., Alexandria, VA 22312

ph: (800) 553-NTIS (6847)

email: orders $a$ ntis.gov <http://www.ntis.gov/about/form.aspx>

Online ordering: http://www.ntis.gov

This document was printed on recycled paper.

(8/2010) 


\title{
Second Line of Defense Master Spares Catalog
}

\author{
DL Henderson RT Brigantic \\ G Muller CJ Perkins \\ TM Mercier SK Cooley
}

November 2012

Prepared for the U.S. Department of Energy

under Contract DE-AC05-76RL01830

Pacific Northwest National Laboratory

Richland, Washington 99352 


\section{Master Parts Catalog}

This catalog is intended to be a comprehensive listing of repair parts, components, kits, and consumable items used on the equipment deployed at Second Line of Defense (SLD) sites worldwide. The catalog covers detection, central alarm station, network, ancillary equipment, and tools and is backed by a Master Parts Database that is used to generate the standard report views. This relational database contains a record for every part in the catalog along with supporting tables for normalizing fields in the records. The database also includes supporting queries, database maintenance forms, and reports.

This catalog consists of the following brief description section followed by a set of separate views of the Master Parts Database. The primary views are:

Nomenclature view. A list of every part in the catalog sorted alphabetically by standard name or nomenclature.

Manufacturer view. A similar list grouped by the part manufacturer.

End item view. Grouped by the major piece of equipment to which the part applies.

Additional views may be appended to the catalog as required.

\section{Definitions}

End item. A final combination of end products, component parts, and/or materials that is ready for its intended use, e.g., an assembled detection system.

Part. A unique, individual item, set, or kit that is a replaceable component of an end item of SLD equipment.

Kit. An assemblage of parts, typically including those needed to install or affix a component to a larger system.

Assembly. An assembled collection of parts installed as single unit.

Board. A circuit board or similar replaceable item installed as a single unit.

Tool. Any general purpose or special item that is required to perform a maintenance action but is not itself a component of an assembly or end item.

\section{Usage and Intent}

The current catalog is a draft aggregation of spares data from various sources. This version should be used only for staffing and limited planning purposes until superseded by an initial operational release, TBD.

Upon an operational release, this manual is intended for use in SLD maintenance and program management activities that address or are impacted by spares ordering, contracting, costing, stocking, sourcing, or analysis. The intent is to provide a comprehensive list that unambiguously identifies all relevant items of program-wide interest. This catalog will facilitate consistent and accurate inventory management, analysis, and decisions. 


\section{Responsibilities}

In the near term, the spares analysis team at the Pacific Northwest National Laboratory (PNNL) will continue to ingest, normalize, and clean the spares database with the aim of releasing an initial operationally useful version of the spares catalog. Ultimately, the spares database will be managed as a single database instance, tentatively as a subcomponent of the SLD Portal.

All SLD Program operators should note discrepancies and alternatives for improving structure, completeness, and data quality with the goal of rapidly maturing the database so that it can be of use throughout the program.

\section{Data Sources}

The existing spares data records were derived from various SLD Sustainability program records including manufacturers data, site master equipment lists, LMP inventories, and purchasing records. Discovery and ingestion of data sources is ongoing. As of this draft catalog version, these sources include:

\section{Table 1. Provenance Elements in Database}

\begin{tabular}{|c|c|}
\hline Provenance & Worksheet Source \\
\hline Aspect Spare Parts Calculator v2.xlsx & Calculation \\
\hline Aspect Spare Parts Calculator v2.xlsx & Telecommunications \\
\hline Aspect Spare Parts Calculator v2.xlsx & Video Monitoring \& Server \\
\hline $\begin{array}{l}\text { Data Acquisition, processing and displaying system. Composition Catalog } \\
\text { Rev.1 }\end{array}$ & Table B.6 \\
\hline Initial Sihanoukville Spare Parts Inventory & Traffic Arms \\
\hline Malaysia, Port Klang Spare Parts List Siemens & OCR \\
\hline Multiple sources & Table B.4 \\
\hline $\begin{array}{l}\text { Spare Parts Recommendations based on Aug-2011 v.2, Make Model } \\
\text { Recommended Spare Purchase file }\end{array}$ & APS OCR \\
\hline $\begin{array}{l}\text { Spare Parts Recommendations based on Aug-2011 v.2, Make Model } \\
\text { Recommended Spare Purchase file }\end{array}$ & HTS OCR \\
\hline $\begin{array}{l}\text { Spare Parts Recommendations based on Aug-2011 v.2, Make Model } \\
\text { Recommended Spare Purchase file }\end{array}$ & SAFEPASS CAS \\
\hline $\begin{array}{l}\text { Spare Parts Recommendations based on Aug-2011 v.2, Make Model } \\
\text { Recommended Spare Purchase file }\end{array}$ & Schneider CAS \\
\hline $\begin{array}{l}\text { Spare Parts Recommendations based on Aug-2011 v.2, Make Model } \\
\text { Recommended Spare Purchase file }\end{array}$ & Serco CAS - 1 Generation Old \\
\hline $\begin{array}{l}\text { Spare Parts Recommendations based on Aug-2011 v.2, Make Model } \\
\text { Recommended Spare Purchase file }\end{array}$ & Serco CAS - 2 Generations Old \\
\hline $\begin{array}{l}\text { Spare Parts Recommendations based on Aug-2011 v.2, Make Model } \\
\text { Recommended Spare Purchase file }\end{array}$ & Serco CAS - 3 Generations Old \\
\hline $\begin{array}{l}\text { Spare Parts Recommendations based on Aug-2011 v.2, Make Model } \\
\text { Recommended Spare Purchase file }\end{array}$ & Serco CAS - Current \\
\hline $\begin{array}{l}\text { Spare Parts Recommendations based on Aug-2011 v.2, Make Model } \\
\text { Recommended Spare Purchase file, Port Klang, Malaysia }\end{array}$ & Siemens Wireless Network \\
\hline $\begin{array}{l}\text { Spare Parts Recommendations based on Aug-2011 v.2, Make Model } \\
\text { Recommended Spare Purchase file, Port Klang, Malaysia. }\end{array}$ & Serco Wireless Network \\
\hline
\end{tabular}




\begin{tabular}{ll}
\hline \multicolumn{1}{c}{ Provenance } & \multicolumn{1}{c}{ Worksheet Source } \\
\hline $\begin{array}{l}\text { Spare Parts Recommendations based on Honduras Spares Inventory 4/16/07 \& } \\
\text { Port of Manzanillo (MX), 12/19/08 }\end{array}$ & ICIS OCR \\
$\begin{array}{l}\text { Spare Parts Recommendations based on Latvia example \& Aug-2011 v.2, Make } \\
\text { Model Recommended Spare Purchase file, Raven Spare Price List }\end{array}$ & RAVEN CAS \\
$\begin{array}{l}\text { Spare Parts Recommendations based on Latvia example \& Draft Typical CORE } \\
\text { Site Spares List (4/28/10); Port of Kaohsiung, Taiwan, Spares Inventory; Port } \\
\text { Klang, Malaysia }\end{array}$ & Utility Panels \\
$\begin{array}{l}\text { Spare Parts Recommendations based on manufacturer spare parts list 2008, SLD } \\
\text { Help Desk/Spare Parts/Thermo Spares List 2012, with PNNL preferred pricing } \\
\text { good through 12-31-12 }\end{array}$ & ThermoFisher \\
$\begin{array}{l}\text { Spare Parts Recommendations based on TSA 2011 recommendations } \\
\text { TSA SparePartsPictorial11-10.pdf }\end{array}$ & TSA \\
Video monitoring system. Composition Catalog Rev.1 & TSA \\
\hline
\end{tabular}

\section{Change Management}

This is a pre-decisional, draft version of the catalog. It nominates several data standards. Every attempt has been made to collect and organize as much relevant spare parts data as possible. This document is for staffing purposes only and will be superseded by an initial operational release. Until the database is placed under formal configuration management, the catalog should not be considered version controlled.

No version marks or consistency between variants will be enforced until an initial operational release, TBD.

\section{Master Parts Catalog Data Definitions}

The data elements defined in this chapter are an initial nominated specification for fields within catalog records.

\section{Nomenclature}

The nomenclature field is a regularized basic name that follows a general-to-specific, comma-separated pattern of at least one and up to three levels:

Basic name. The fundamental function or most general useful name for an item, e.g., CABLE

Basic name modifier. A modifier to the basic name that makes the function or description more complete, a type, e.g., USB

Basic name extended modifier. An extension that distinguishes the item from similar items or makes its purpose clear, e.g., 6 FT

The nomenclature field is backed by three tables in the spares database. These tables are used to construct the nomenclature from regularized components, e.g., CABLE, USB, 6FT. Nomenclature is spelled out entirely in capital letters.

\section{Unit of Issue}

Unit of issue is the count of the item that is provided by the catalog entry. Most items have basis of issue EA meaning each, which denotes a single item. The current unit of issue codes are defined in Table 2. 
Table 2. Unit of Issue Codes

\begin{tabular}{ll}
\hline \multicolumn{1}{c}{ Code } & \multicolumn{1}{c}{ Explanation } \\
\hline EA & each \\
KIT & kit \\
SET & set \\
HND & hundred count \\
GRS & gross \\
DOZ & twelve count \\
GAL & US gallon \\
4 PCS & 4 pieces \\
12 PCS & 12 pieces \\
16 PCS & 16 pieces \\
24 PCS & 24 pieces \\
\hline
\end{tabular}

\section{Cost}

Cost data are maintained in current fiscal year U.S. dollars. The quality of the existing cost data vary widely depending on the source for the catalog item. Cost data are presented in this version for staffing and rough estimates only. Ultimately, cost data should reflect an authoritative planning figure, but the contract cost to deliver a particular part to a particular point of need may vary substantially from the figure in the database. Depending on the situation, it may be useful to store site- or LMP-specific costs for spare parts in a separate database linked to inventory at that site or LMP.

\section{Basis of Stockage Level}

The basis of stockage level is the basis against which a stockage level for the part should be calculated. The basis of stockage level is paired with a quantity against which to apply the basis. For example, a part that has a basis of per 10 systems and a quantity of two should be stocked in a quantity of two per 10 systems supported by the stocking maintenance activity. The basis of stockage level and quantity data are intended for use in a business logic that accounts for rounding, minimums, and restrictions on stockage. The existing values for basis of issue are defined in Table 3.

Table 3. Basis of Stockage Level Codes

\begin{tabular}{ll}
\hline \multicolumn{1}{c}{ Code } & \multicolumn{1}{c}{ Explanation } \\
\hline 10 Systems & The basis of issue is quantity per 10 supported end item systems \\
10 CAS & The basis of issue is quantity per 10 supported CAS systems \\
Site & The basis of issue is quantity per supported site \\
None & No basis of issue, do not stock this as a spare against a basis of issue \\
10 OCR & The basis of issue is quantity per 10 supported OCR Systems \\
10 Traffic Arms & The basis of issue is quantity per 10 supported traffic arms \\
Each & The basis of issue is quantity per end item supported \\
1 System & The basis of issue is quantity per 1 supported end item system \\
\hline
\end{tabular}

\subsubsection{Recoverability Code}

The recoverability code provides guidance on the disposition of a failed part and the level at which parts may be disposed, determined to be economically repairable, and repaired for return to inventory. The recoverability codes are defined in Table 4. 
Table 4. Recoverability Codes

\begin{tabular}{cll}
\hline Code & \multicolumn{1}{c}{ Explanation } \\
\hline Z & $\begin{array}{l}\text { Non-recoverable; dispose of at LMP/Operator level when } \\
\text { unserviceable }\end{array}$ \\
D & Recoverable; at manufacturer level \\
I & Recoverable; at intermediate level \\
O & Recoverable; at LMP level \\
X & No recoverability guidance for this part \\
\hline
\end{tabular}

\subsubsection{Special Handling Code}

Special handing codes provide guidance for parts that require special handling or are currently being recovered under special circumstances, for example for failure analysis. The special handling codes are defined in Error! Reference source not found.

Table 5. Special Handling Codes

\begin{tabular}{cl}
\hline Code & \multicolumn{1}{c}{ Explanation } \\
\hline M & Item is high value or contains high value components \\
H & Item contains hazardous material \\
F & Item should be recovered under special instruction for failure analysis \\
MH & High value and hazardous material \\
MF & High value and failure analysis required \\
HF & Hazardous material and failure analysis required \\
MHF & High value, hazardous material, and failure analysis required \\
X & No special handling required \\
\hline
\end{tabular}

\subsubsection{Obsolescence Code}

The obsolescence code is intended as a way to call out parts that are nearing end of life and will become difficult to source. It can also be used for parts that may remain in service, but that have become obsolete and will be replaced by a different part or through a system upgrade. A special case of obsolescence coding is the distinction between potted and non-potted versions of high-voltage detector components. Both versions exist in inventory and the version can be distinguished by manufacturer's part number. As the retrofit to potted parts is completed, these codes may no longer be needed. The obsolescence codes are defined in Error! Reference source not found..

Table 6. Obsolescence Codes

\begin{tabular}{c|l}
\hline Code & \multicolumn{1}{c}{ Explanation } \\
\hline X & No obsolescence information available \\
A & Active \\
O & Obsolete \\
E & Nearing end of life \\
P & Potted version \\
NP & Non potted version \\
\hline
\end{tabular}




\section{Master Parts Catalog by Nomenclature}

\begin{tabular}{|c|c|c|c|c|c|c|c|c|c|}
\hline Nomenclature & Manufacturer & Part Number & UI & Basis per & $\mathrm{BQ}$ & Cost & $\mathrm{SH}$ & $\mathrm{R}$ & $\mathrm{O}$ \\
\hline & Aspect & PS-3 & EA & & & & $x$ & $x$ & $x$ \\
\hline & Aspect & SVP-17 & EA & & & & $x$ & $x$ & $x$ \\
\hline & Aspect & 426429.004 & EA & & & & $x$ & $x$ & $x$ \\
\hline & Aspect & BP12-12 & EA & & & & $x$ & $x$ & $x$ \\
\hline & Aspect & UPS-03 & EA & & & & $x$ & $x$ & $x$ \\
\hline & Aspect & Moscow M1-30 & EA & & & & $x$ & $x$ & $x$ \\
\hline & TSA & 6692 & EA & & & & $x$ & $x$ & 0 \\
\hline & TSA & 8453 & EA & & & & $x$ & $x$ & $x$ \\
\hline & TSA & $8870 \mathrm{~A}$ & EA & & & & $x$ & $x$ & $x$ \\
\hline ACCESSORY, CABLE & $A D C$ & ADCCMVIB-3CB20-4 & EA & 10 Systems & 4 & $\$ 666.68$ & $x$ & $x$ & $x$ \\
\hline ACCESSORY, TRAFFIC ARM & Magnetic Autocontrol & $3004-0001$ & EA & 10 Traffic Arms & 40 & $\$ 5.00$ & $x$ & $x$ & $x$ \\
\hline ADAPTER & ThermoFisher & GOVC000493 & KIT & & 1 & $\$ 77.21$ & $x$ & $x$ & $x$ \\
\hline ADAPTER, INTERFACE, IP CONSOLE & Hewlett Packard & & EA & Site & $1-2$ & $\$ 99.00$ & $x$ & $x$ & $x$ \\
\hline ADAPTER, POLE & Pelco & PA101 & EA & 10 Systems & 1 & $\$ 190.00$ & $x$ & $x$ & $x$ \\
\hline ADAPTER, POLE & Pelco & 0461B & EA & 10 CAS & 1 & $\$ 70.00$ & $x$ & $x$ & $x$ \\
\hline ADAPTER, PROTECTIVE & ThermoFisher & MEVE339 & KIT & & 1 & $\$ 134.57$ & $x$ & $x$ & $x$ \\
\hline AIR CONDITIONER & ISC Sales & NE03012604BX0 & EA & 10 Systems & 1 & $\$ 2,000.00$ & M & $x$ & $x$ \\
\hline AIR CONDITIONER, XMHC & ThermoFisher & VEIN93 & KIT & & 1 & $\$ 3,137.80$ & M & $x$ & $x$ \\
\hline ALARM, SOUND & Aspect & 000002.004 & $\mathrm{EA}$ & 1 system & & $\$ 60.00$ & $x$ & $x$ & $x$ \\
\hline ALARM, VISUAL & Aspect & 000002.008 & EA & 1 system & & $\$ 60.00$ & $x$ & $x$ & $x$ \\
\hline ALARM, VISUAL & Aspect & 000002.007 & EA & 1 system & & $\$ 60.00$ & $x$ & $x$ & $x$ \\
\hline ALARM, VISUAL & Aspect & 000002.006 & EA & 1 system & & & $x$ & $x$ & $x$ \\
\hline ALARM, VISUAL & Aspect & 000002.005 & EA & 1 system & & & $x$ & $x$ & $x$ \\
\hline
\end{tabular}




\begin{tabular}{|c|c|c|c|c|c|c|c|c|c|}
\hline Nomenclature & Manufacturer & Part Number & UI & Basis per & $B Q$ & Cost & $\mathrm{SH}$ & $\mathrm{R}$ & 0 \\
\hline AMPLIFIER, NEUTRON CHANNEL & Aspect & $418249.006-01$ & EA & 1 system & & $\$ 1,477.00$ & $x$ & $x$ & $x$ \\
\hline AMPLIFIER, NEUTRON CHANNEL & Aspect & 418249.006 & EA & 1 system & & $\$ 1,477.00$ & $x$ & $x$ & $x$ \\
\hline AMPLIFIER, NEUTRON CHANNEL & Aspect & & EA & 1 system & & $\$ 1,477.00$ & $x$ & $x$ & $x$ \\
\hline ANTENNA TERMINATOR, $50 \mathrm{OHM}$ & Siemens & TI 795-1R & EA & 10 Systems & 2 & $\$ 398.00$ & $x$ & $x$ & $x$ \\
\hline ANTENNA, 26GHZ, 0.3M & Dragonwave & VHLP1-26-DW1 & EA & 10 Systems & 1 & $\$ 450.00$ & $x$ & $x$ & $x$ \\
\hline ANTENNA, 28GHZ, 0.3M & Dragonwave & VHLP1-28-DW1 & EA & 10 Systems & 1 & $\$ 450.00$ & $x$ & $x$ & $x$ \\
\hline ANTENNA, 28GHZ, 0.6M & Dragonwave & VHLP2-28-DW1 & EA & 10 Systems & 1 & $\$ 459.00$ & $x$ & $x$ & $x$ \\
\hline ANTENNA, EXTERNAL & D-Link & $0465 \mathrm{~A}$ & EA & $10 \mathrm{CAS}$ & 1 & $\$ 150.00$ & $x$ & $x$ & $x$ \\
\hline ASSEMBLY, CABLE HARNESS, EXT TX & ThermoFisher & YP11702228B & KIT & & 1 & $\$ 182.12$ & $x$ & $x$ & $x$ \\
\hline ASSEMBLY, CABLE HARNESS, EXT TX & ThermoFisher & YP11702230B & KIT & & 1 & $\$ 182.12$ & $x$ & $x$ & $x$ \\
\hline ASSEMBLY, CABLE HARNESS, EXT TX & ThermoFisher & YP11702230A & KIT & & 1 & $\$ 182.12$ & $x$ & $x$ & $x$ \\
\hline ASSEMBLY, CABLE HARNESS, EXT TX & ThermoFisher & YP11702228A & KIT & & 1 & $\$ 182.12$ & $x$ & $x$ & $x$ \\
\hline ASSEMBLY, CABLE, 12 VDC FAN & ThermoFisher & YP11702479 & KIT & & 3 & $\$ 62.73$ & $x$ & $x$ & $x$ \\
\hline ASSEMBLY, JUNCTION BOX & ThermoFisher & GOVA000102 & KIT & & 1 & $\$ 2,288.10$ & M & $x$ & $x$ \\
\hline ASSEMBLY, LH, RX(B) SENSOR & ThermoFisher & YP11702251-1 & KIT & & 1 & $\$ 540.14$ & $x$ & $x$ & $x$ \\
\hline ASSEMBLY, LH, TX(A) SENSOR & ThermoFisher & YP11702251 & KIT & & 1 & $\$ 540.14$ & $x$ & $x$ & $x$ \\
\hline ASSEMBLY, MEDIA CONVERTER & TSA & 8590 & EA & 10 Systems & 1 & $\$ 552.00$ & $x$ & $x$ & $x$ \\
\hline ASSEMBLY, NAI, COMPLETE & ThermoFisher & YP11702006 & KIT & & 2 & $\$ 18,322.53$ & M & $x$ & $x$ \\
\hline ASSEMBLY, RH, TX(B) SENSOR & ThermoFisher & YP11702250 & KIT & & 1 & $\$ 540.14$ & $x$ & $x$ & $x$ \\
\hline ASSEMBLY, SURGE ARRESTOR, 40A 120V & ThermoFisher & MEVE367 & KIT & & 1 & $\$ 273.85$ & $x$ & $x$ & $x$ \\
\hline BATTERY & Aspect & 000003.001 & EA & 1 system & & $\$ 30.00$ & $x$ & $x$ & $x$ \\
\hline BATTERY & SONNENSCHEIN & A512/10.0S & EA & & & & $\mathrm{H}$ & $x$ & $x$ \\
\hline
\end{tabular}




\begin{tabular}{|c|c|c|c|c|c|c|c|c|c|}
\hline Nomenclature & Manufacturer & Part Number & UI & Basis per & $B Q$ & Cost & $\mathrm{SH}$ & $\mathrm{R}$ & $\mathrm{O}$ \\
\hline BATTERY PACK & APC & JB0829023294 & EA & 10 Systems & 1 & $\$ 400.00$ & $\mathrm{H}$ & $x$ & $x$ \\
\hline BATTERY PACK, 192V, RM & APC & SURT192MXLBP & EA & 10 Systems & 1 & $\$ 718.95$ & $\mathrm{H}$ & $x$ & $x$ \\
\hline BATTERY PACK, 24V, RS XS & APC & BR24BP & EA & 10 Systems & 1 & $\$ 131.58$ & $\mathrm{H}$ & $x$ & $x$ \\
\hline BATTERY, 18AH 12V & TSA & $6456 \mathrm{~A}$ & EA & 10 Systems & 0 & $\$ 90.00$ & $\mathrm{H}$ & $x$ & $x$ \\
\hline BATTERY, 26AH 12V & TSA & $6456 B$ & EA & 10 Systems & 1 & $\$ 90.00$ & $\mathrm{H}$ & $x$ & $x$ \\
\hline BATTERY, BACKUP UNIT & Weidmuller & 9916280012 & EA & 10 Systems & 2 & $\$ 600.00$ & $\mathrm{H}$ & $x$ & 0 \\
\hline BOARD & Aspect & & EA & & & & $x$ & $x$ & $x$ \\
\hline BOARD & TSA & 8753 & EA & & & & $x$ & $x$ & $x$ \\
\hline BOARD & TSA & 8306 & EA & & & & $x$ & $x$ & $x$ \\
\hline BOARD, 8403 & TSA & 8403AP & EA & 10 Systems & V & $\$ 147.50$ & $x$ & $x$ & $x$ \\
\hline BOARD, 8453 & TSA & $8453 G$ & EA & 10 Systems & 2 & $\$ 235.50$ & $x$ & $x$ & $x$ \\
\hline BOARD, CONTROLLER, BATTERY & ThermoFisher & $5681 \mathrm{~A}$ & KIT & & 2 & $\$ 431.02$ & $x$ & $x$ & $x$ \\
\hline BOARD, COVERSION, 24V DC DIGITAL IO & ThermoFisher & CONV1 & KIT & & 1 & $\$ 138.84$ & $x$ & $x$ & $x$ \\
\hline BOARD, DISPLAY & ThermoFisher & GOVC000167 & KIT & & 1 & $\$ 89.32$ & $x$ & $x$ & $x$ \\
\hline BOARD, MAIN CONTROL & ThermoFisher & GoVC000172 & KIT & & 1 & $\$ 1,471.75$ & M & $x$ & $x$ \\
\hline BOARD, SC-771 & TSA & $8751 \mathrm{AA}$ & EA & 10 Systems & 1 & $\$ 675.00$ & $x$ & $x$ & $x$ \\
\hline BOARD, SCA-774 & TSA & 8754 & EA & 10 Systems & V & $\$ 650.00$ & $x$ & $x$ & $x$ \\
\hline BOARD, SINGLE, IO TO ETHERNET & APS Technology & 24-016-015 & EA & 10 OCR & 1 & $\$ 644.00$ & $x$ & $x$ & $x$ \\
\hline BOARD, SWITCH & Aspect & 685179.011 & EA & 1 system & & $\$ 699.00$ & $x$ & $x$ & $x$ \\
\hline BOARD, SWITCH & Aspect & 685179.009 & EA & 1 system & & $\$ 699.00$ & $x$ & $x$ & $x$ \\
\hline BOARD, SWITCH & Aspect & 685179.004-01 & EA & 1 system & & $\$ 340.00$ & $x$ & $x$ & $x$ \\
\hline BOARD, SWITCH & Aspect & & EA & 1 system & & $\$ 352.00$ & $x$ & $x$ & $x$ \\
\hline
\end{tabular}




\begin{tabular}{|c|c|c|c|c|c|c|c|c|c|}
\hline Nomenclature & Manufacturer & Part Number & UI & Basis per & $B Q$ & Cost & $\mathrm{SH}$ & $\mathrm{R}$ & $\mathrm{O}$ \\
\hline BOARD, SWITCH & Aspect & 685179.004 & EA & 1 system & & $\$ 699.00$ & $x$ & $x$ & $x$ \\
\hline BOARD, SWITCH & Aspect & 685169.003 & EA & 1 system & & $\$ 699.00$ & $x$ & $x$ & $x$ \\
\hline BOARD, SWITCH & Aspect & 685179.011-01 & EA & 1 system & & $\$ 699.00$ & $x$ & $x$ & $x$ \\
\hline BOOM , 12 FT, SWING AWAY & Magnetic Autocontrol & MSB-035 & EA & 10 Traffic Arms & 2 & $\$ 200.00$ & $x$ & $x$ & $x$ \\
\hline BOOM , 15 FT & Magnetic Autocontrol & MSB 5N-045 & EA & 10 Traffic Arms & 2 & $\$ 174.00$ & $x$ & $x$ & $x$ \\
\hline BRACKET & TSA & 3450 & EA & & & & $x$ & $x$ & $x$ \\
\hline BRACKET, CAMERA, POLE MOUNT & Pelco & EM2000 & EA & 10 Systems & 1 & $\$ 49.50$ & $x$ & $x$ & $x$ \\
\hline CABINET & Aspect & $301445.032-03$ & EA & 1 system & & $\$ 5,795.00$ & $x$ & $x$ & $x$ \\
\hline CABINET & Aspect & 301445.018 & EA & 1 system & & $\$ 6,417.00$ & $x$ & $x$ & $x$ \\
\hline CABINET & Aspect & & EA & 1 system & & $\$ 2,000.00$ & $x$ & $x$ & $x$ \\
\hline CABINET & Aspect & $301445.032-02$ & EA & 1 system & & $\$ 5,795.00$ & $x$ & $x$ & $x$ \\
\hline CABINET & Aspect & 301445.028 & EA & 1 system & & $\$ 6,969.00$ & $x$ & $x$ & $x$ \\
\hline CABINET & Aspect & 418252.002 & EA & 1 system & & $\$ 6,969.00$ & $x$ & $x$ & $x$ \\
\hline CABINET & Aspect & $418252.002-01$ & EA & 1 system & & $\$ 6,969.00$ & $x$ & $x$ & $x$ \\
\hline CABINET, SERVER & Advantech & ADAM-4572 & EA & & & & $x$ & $x$ & $x$ \\
\hline CABINET, SERVER & Aspect & 426441.001 & EA & & & & $x$ & $x$ & $x$ \\
\hline CABINET, SERVER & Aspect & I-7513 & EA & & & & $x$ & $x$ & $x$ \\
\hline CABINET, SERVER & Aspect & 425543.004 & EA & & & & $x$ & $x$ & $x$ \\
\hline CABINET, TS NEMA 4 & Rittal & TS 8886641 & EA & 10 Systems & 1 & $\$ 5,700.00$ & $M$ & $x$ & $x$ \\
\hline CABLE & Tripp Lite & & EA & Site & 1 & $\$ 40.00$ & $x$ & $x$ & $x$ \\
\hline CABLE & TSA & 0360 & EA & & & & $x$ & $x$ & $x$ \\
\hline CABLE & TSA & 0360A & EA & & & & $x$ & $x$ & $x$ \\
\hline
\end{tabular}




\begin{tabular}{|c|c|c|c|c|c|c|c|c|c|}
\hline Nomenclature & Manufacturer & Part Number & UI & Basis per & $\mathrm{BQ}$ & Cost & $\mathrm{SH}$ & $\mathrm{R}$ & $\mathrm{O}$ \\
\hline CABLE & TSA & 0344D & EA & & & & $x$ & $x$ & $x$ \\
\hline CABLE & TSA & $0301 C$ & EA & & & & $x$ & $x$ & $x$ \\
\hline CABLE & TSA & 0360B & EA & & & & $x$ & $x$ & $x$ \\
\hline CABLE & TSA & $2802 B$ & EA & & & & $x$ & $x$ & $x$ \\
\hline CABLE & TSA & 0301E & EA & & & & $x$ & $x$ & $x$ \\
\hline CABLE, 12 PAIR CROSSOVER, 1000 FT & Quabbin Wire \& Cable & 8525 & EA & 10 CAS & 1 & $\$ 2,000.00$ & $\mathrm{M}$ & $x$ & $x$ \\
\hline CABLE, 2 CONDUCTOR 18AWG, 100 FT & TSA & & EA & 10 CAS & 1 & $\$ 50.00$ & $x$ & $x$ & $x$ \\
\hline CABLE, CAT5E & Belkin & UTP 4 pairs Cat5e & EA & 10 Systems & 1 & $\$ 30.00$ & $x$ & $x$ & $x$ \\
\hline CABLE, CAT5E, RJ-45 & Belkin & & EA & 10 Systems & 1 & $\$ 30.00$ & $x$ & $x$ & $x$ \\
\hline CABLE, CAT6 PLUS, 7 FT & Leviton & 6246007L & EA & 10 Systems & 4 & $\$ 24.36$ & $x$ & $x$ & $x$ \\
\hline CABLE, CAT6, 2.13M & Prolink & R5032XGN & EA & 10 Systems & 4 & $\$ 16.96$ & $x$ & $x$ & $x$ \\
\hline CABLE, CL, BFR/SPL TO SURGE PROTECTOR & ThermoFisher & YP11702499 & KIT & & 1 & $\$ 30.71$ & $x$ & $x$ & $x$ \\
\hline CABLE, COAX & TSA & 0352 & 12 PCS & 10 Systems & 1 & $\$ 405.00$ & $x$ & $x$ & $x$ \\
\hline CABLE, COAX & TSA & 0349 & 4 PCS & 10 Systems & 1 & $\$ 255.00$ & $x$ & $x$ & $\mathrm{x}$ \\
\hline CABLE, COAX & TSA & 0350 & 16 PCS & 10 Systems & 1 & $\$ 543.00$ & $x$ & $x$ & $x$ \\
\hline CABLE, COAX & TSA & 0353 & 24 PCS & 10 Systems & 1 & $\$ 780.00$ & $x$ & $x$ & $x$ \\
\hline CABLE, DC POWER, BOTTOM FAN & ThermoFisher & YP11702488 & KIT & & 1 & $\$ 47.85$ & $x$ & $x$ & $x$ \\
\hline CABLE, DC POWER, TOP FAN & ThermoFisher & YP11702487 & KIT & & 1 & $\$ 46.40$ & $x$ & $x$ & $x$ \\
\hline CABLE, DUPLEX, LC SC 2M & FIS & D3LYS2FISC & EA & 10 Systems & 2 & $\$ 41.28$ & $x$ & $x$ & $x$ \\
\hline CABLE, EXTENDER, KVM & Tripp Lite & & EA & Site & 1 & $\$ 230.00$ & $x$ & $x$ & $x$ \\
\hline CABLE, FC SC SM, 3M & ICIS & FCSC-SMD-02 & EA & $10 \mathrm{OCR}$ & 2 & $\$ 17.39$ & $x$ & $x$ & $x$ \\
\hline CABLE, FIBER OPTIC, 1M & Leviton & Cat\#62DSC-M01 & EA & 10 Systems & 1 & $\$ 20.00$ & $x$ & $x$ & $x$ \\
\hline
\end{tabular}




\begin{tabular}{|c|c|c|c|c|c|c|c|c|c|}
\hline Nomenclature & Manufacturer & Part Number & UI & Basis per & $B Q$ & Cost & $\mathrm{SH}$ & $\mathrm{R}$ & $\mathrm{O}$ \\
\hline CABLE, FIBER OPTIC, 3M & Leviton & Cat\#62DSC-M03 & EA & 10 Systems & 1 & $\$ 30.00$ & $x$ & $x$ & $\mathrm{x}$ \\
\hline CABLE, FIBER, 30 FT SC & ThermoFisher & YP11702523 & KIT & & 1 & $\$ 86.65$ & $x$ & $x$ & $x$ \\
\hline CABLE, FIBER, CONV TO COUPLER & ThermoFisher & YP11702500 & KIT & & 1 & $\$ 54.01$ & $x$ & $x$ & $x$ \\
\hline CABLE, LAN, POE SW RS485 LAN CON & ThermoFisher & YP11702497 & KIT & & 1 & $\$ 29.12$ & $x$ & $x$ & $x$ \\
\hline CABLE, LAN, POE SW TO TEMP BS & ThermoFisher & YP11702496 & KIT & & 1 & $\$ 29.12$ & $x$ & $x$ & $x$ \\
\hline CABLE, LC SC SM, 2M & ICIS & FCSC-SMD-02 & EA & 10 OCR & 5 & $\$ 11.97$ & $x$ & $x$ & $x$ \\
\hline CABLE, LC SC SM, 3M & ICIS & 13-HPS108-3M & EA & 10 OCR & 4 & $\$ 9.26$ & $x$ & $x$ & $x$ \\
\hline CABLE, MOLEX, CAT5E & Generic Manufacturer & PCD00174BK & EA & 10 Systems & 4 & $\$ 8.00$ & $x$ & $x$ & $x$ \\
\hline CABLE, MOLEX, CAT5E & Generic Manufacturer & PCD00177BK & EA & 10 Systems & 4 & $\$ 7.92$ & $x$ & $x$ & $x$ \\
\hline $\begin{array}{l}\text { CABLE, PC/PLC PROGRAM, 9PIN SERIAL TO } \\
\text { RJ45 }\end{array}$ & Automation Direct & D2-DSCBL & EA & 10 Systems & 1 & $\$ 25.00$ & $x$ & $x$ & $x$ \\
\hline CABLE, POWER, 16A 230V & APC & AP9876 & EA & 10 Systems & 4 & $\$ 151.56$ & $x$ & $x$ & $x$ \\
\hline CABLE, POWER, 3 FT & Generic Manufacturer & NA & EA & 10 Systems & 1 & $\$ 30.00$ & $x$ & $x$ & $x$ \\
\hline CABLE, POWER, 48 VDC TO MON PCB & ThermoFisher & YP11702492 & KIT & & 1 & $\$ 33.86$ & $x$ & $x$ & $x$ \\
\hline CABLE, POWER, AC & APC & AP9870 & EA & 10 Systems & 2 & $\$ 29.48$ & $x$ & $x$ & $x$ \\
\hline CABLE, POWER, IEC MALE TO FEMALE & PI & $7303 B$ & EA & 10 CAS & 1 & $\$ 6.00$ & $x$ & $x$ & $x$ \\
\hline CABLE, POWER, IN TO TB & ThermoFisher & YP11702489 & KIT & & 1 & $\$ 34.50$ & $x$ & $x$ & $x$ \\
\hline CABLE, POWER, MON PCB TO 48 VDC TB & ThermoFisher & YP11702494 & KIT & & 1 & $\$ 37.22$ & $x$ & $x$ & $x$ \\
\hline CABLE, POWER, TB TO 48 VDC PS & ThermoFisher & YP11702490 & KIT & & 1 & $\$ 50.75$ & $x$ & $x$ & $x$ \\
\hline CABLE, SC SC SM, 5M & ICIS & SCSSTG16 & EA & $10 \mathrm{OCR}$ & 5 & $\$ 22.00$ & $x$ & $x$ & $x$ \\
\hline CABLE, SMB, MALE & HTS & 550054 & EA & $10 \mathrm{OCR}$ & 6 & $\$ 30.00$ & $x$ & $x$ & $x$ \\
\hline CAMERA & Sony & 0460 & EA & 10 CAS & 1 & $\$ 1,594.05$ & M & $x$ & $\mathrm{O}$ \\
\hline CAMERA, DIGITAL, SURVEILLANCE & Sony & SNC-Z2ON & EA & 10 Systems & 1 & $\$ 915.00$ & $x$ & $x$ & 0 \\
\hline CAMERA, NETWORK & Axis & Axis P1343 & EA & & & & $x$ & $x$ & $x$ \\
\hline
\end{tabular}




\begin{tabular}{|c|c|c|c|c|c|c|c|c|c|}
\hline Nomenclature & Manufacturer & Part Number & UI & Basis per & $B Q$ & Cost & $\mathrm{SH}$ & $\mathrm{R}$ & $\mathrm{O}$ \\
\hline CAMERA, NETWORK & Sarix & AAM-CROO & EA & 10 Systems & 1 & $\$ 1,200.00$ & M & $x$ & $\mathrm{x}$ \\
\hline CAMERA, NETWORK & TSA & 0460C & EA & 10 CAS & 1 & $\$ 1,594.05$ & M & $x$ & $x$ \\
\hline $\begin{array}{l}\text { CAMERA, NETWORK, COLOR 1.4MP MEGA } \\
\text { RESOLUTION }\end{array}$ & Lumenera & & EA & $10 \mathrm{OCR}$ & 3 & $\$ 2,200.00$ & M & $x$ & $x$ \\
\hline CAMERA, REAR & Sony & 17COLREAR06ASSY & EA & 10 OCR & 1 & $\$ 930.00$ & $x$ & $x$ & $x$ \\
\hline CAMERA, SCH-2, YELLOW & Sony & 19BWOR3712500EL & EA & 10 OCR & 2 & $\$ 1,040.00$ & M & $x$ & $x$ \\
\hline CAMERA, SECURITY & Nuvico & Nuvico CV-STD21P & EA & & & & $x$ & $x$ & $x$ \\
\hline CAMERA, SIDE & Sony & 17COL000440ASSY & EA & 10 OCR & 2 & $\$ 900.00$ & $x$ & $x$ & $x$ \\
\hline CAMERA, TOP & Sony & 17COLTOP044ASSY & EA & $10 \mathrm{OCR}$ & 3 & $\$ 930.00$ & $x$ & $x$ & $x$ \\
\hline CAMERA, TOP LENS MOUNTED, 3.0MP & APS Technology & 29-0019-001 & EA & 10 OCR & 1 & $\$ 1,548.00$ & M & $x$ & $x$ \\
\hline CAMERA, VIDEO & Honeywell & Honeywell HZC-755PX & EA & & & & $x$ & $x$ & $x$ \\
\hline CAMERA, VIDEO & Honeywell & $\begin{array}{l}\text { Honeywell HCC- } \\
\text { 745PTW }\end{array}$ & EA & & & & $x$ & $x$ & $x$ \\
\hline CAMERA, VIDEO & Honeywell & Honeywell GC-655P & EA & & & & $x$ & $x$ & $x$ \\
\hline CARD, UPS SNMP & AEG & & EA & $10 \mathrm{OCR}$ & 2 & $\$ 157.87$ & $x$ & $x$ & $x$ \\
\hline CHARGER, 4A 15V & TSA & $6463 B$ & EA & 10 Systems & 1 & $\$ 52.50$ & $x$ & $x$ & $\mathrm{x}$ \\
\hline CHARGER, 5A 15V & TSA & $6463 C$ & EA & 10 Systems & 1 & $\$ 87.50$ & $x$ & $x$ & $\mathrm{x}$ \\
\hline CHARGER, BATTERY, 24V DC & ThermoFisher & GOVC000166 & KIT & & 1 & $\$ 1,139.63$ & M & $\mathrm{x}$ & $\mathrm{x}$ \\
\hline CHASSIS, 650W 80 PLUS POWER SUPPLY & Hewlett Packard & GM505AV & EA & Site & $1-2$ & $\$ 158.00$ & $\mathrm{x}$ & $x$ & $\mathrm{x}$ \\
\hline CHASSIS, 650W 80 PLUS POWER SUPPLY & Hewlett Packard & GM505AV & EA & Site & 1 & $\$ 158.00$ & $\mathrm{x}$ & $x$ & $\mathrm{x}$ \\
\hline $\begin{array}{l}\text { CHASSIS, 650W 85\% EFFICIENCY POWER } \\
\text { SUPPLY }\end{array}$ & Hewlett Packard & & EA & Site & 1 & $\$ 3,079.00$ & M & $x$ & $\mathrm{x}$ \\
\hline $\begin{array}{l}\text { CHASSIS, 650W 85\% EFFICIENCY POWER } \\
\text { SUPPLY }\end{array}$ & Hewlett Packard & & EA & Site & $1-2$ & $\$ 3,079.00$ & $M$ & $x$ & $x$ \\
\hline
\end{tabular}




\begin{tabular}{|c|c|c|c|c|c|c|c|c|c|}
\hline Nomenclature & Manufacturer & Part Number & UI & Basis per & $B Q$ & Cost & $\mathrm{SH}$ & $\mathrm{R}$ & $\mathrm{O}$ \\
\hline $\begin{array}{l}\text { CHASSIS, 650W 85\% EFFICIENCY POWER } \\
\text { SUPPLY }\end{array}$ & Hewlett Packard & & EA & Site & 1 & $\$ 3,079.00$ & M & $x$ & $x$ \\
\hline CHASSIS, SERVER, PROLIANT & Hewlett Packard & 391835-B21 & EA & Site & 1 & $\$ 441.57$ & $x$ & $x$ & $x$ \\
\hline CHASSIS, SERVER, PROLIANT & Hewlett Packard & $371293-405$ & EA & Site & 1 & $\$ 338.00$ & $x$ & $x$ & $x$ \\
\hline CHASSIS, SERVER, PROLIANT & Hewlett Packard & DL380G6 & EA & Site & 1 & $\$ 1,032.00$ & M & $x$ & $x$ \\
\hline CHASSIS, SERVER, PROLIANT & Hewlett Packard & $371293-405$ & EA & Site & $1-2$ & $\$ 338.10$ & $x$ & $x$ & $x$ \\
\hline CHASSIS, SERVER, PROLIANT & Hewlett Packard & $371293-405$ & EA & Site & $1-2$ & $\$ 338.10$ & $x$ & $x$ & $x$ \\
\hline CHASSIS, SERVER, PROLIANT & Hewlett Packard & $371293-405$ & EA & Site & 1 & $\$ 338.00$ & $x$ & $x$ & $x$ \\
\hline CHASSIS, SERVER, PROLIANT & Hewlett Packard & DL380G6 & EA & Site & $1-2$ & $\$ 1,032.00$ & M & $x$ & $x$ \\
\hline CIRCUIT BREAKER & TSA & $7802 B$ & EA & 10 CAS & 1 & $\$ 34.00$ & $x$ & $x$ & $x$ \\
\hline CIRCUIT BREAKER, 1P 120 VAC, 10A & ThermoFisher & FUCB24 & KIT & & 1 & $\$ 122.55$ & $x$ & $x$ & $x$ \\
\hline CIRCUIT BREAKER, 1P 120 VAC, 15A & ThermoFisher & FUCB25 & KIT & & 1 & $\$ 122.55$ & $x$ & $x$ & $\mathrm{x}$ \\
\hline CIRCUIT BREAKER, 1P 120 VAC, 20A & ThermoFisher & FUCB26 & KIT & & 1 & $\$ 122.55$ & $x$ & $x$ & $x$ \\
\hline CIRCUIT BREAKER, 1P 120 VAC, 2A & ThermoFisher & FUCB22 & KIT & & 1 & $\$ 122.55$ & $x$ & $x$ & $x$ \\
\hline CIRCUIT BREAKER, 1P 120 VAC, 5A & ThermoFisher & FUCB23 & KIT & & 1 & $\$ 122.55$ & $x$ & $x$ & $x$ \\
\hline CIRCUIT BREAKER, 1P 230V AC, 20 & Allen Bradley & S201U-K20 & EA & 10 Systems & 1 & $\$ 25.00$ & $x$ & $x$ & $\mathrm{x}$ \\
\hline CIRCUIT BREAKER, 1P 230V AC, 40A & Allen Bradley & S201U-K40 & EA & 10 Systems & 1 & $\$ 50.00$ & $x$ & $x$ & $x$ \\
\hline CIRCUIT BREAKER, 1P 230V AC, 50A & Allen Bradley & S201U-K50 & EA & 10 Systems & 1 & $\$ 60.00$ & $x$ & $x$ & $x$ \\
\hline CIRCUIT BREAKER, 1P DIN RAIL MOUNT, 6A & Allen Bradley & 1492-SP1C060 & EA & 10 Systems & 1 & $\$ 40.00$ & $x$ & $x$ & $x$ \\
\hline CIRCUIT BREAKER, 1P PLUS NEUTRAL, 20A & Allen Bradley & 1492-SP1B200-N & EA & 10 Systems & 1 & $\$ 40.00$ & $x$ & $x$ & $x$ \\
\hline CIRCUIT BREAKER, 1P, 10A & Allen Bradley & 1492-SP1C100 & EA & 10 Systems & 1 & $\$ 40.00$ & $x$ & $x$ & $x$ \\
\hline CIRCUIT BREAKER, 2P, 40A & $A B B$ & F2020AC-40/0.03 & EA & 10 Systems & 1 & & $x$ & $x$ & $x$ \\
\hline CIRCUIT BREAKER, 2P, 50A & Allen Bradley & $1492-S P 2 C 500$ & EA & 10 Systems & 1 & $\$ 120.00$ & $x$ & $x$ & $x$ \\
\hline
\end{tabular}




\begin{tabular}{|c|c|c|c|c|c|c|c|c|c|}
\hline Nomenclature & Manufacturer & Part Number & UI & Basis per & $B Q$ & Cost & $\mathrm{SH}$ & $\mathrm{R}$ & $\mathrm{O}$ \\
\hline CIRCUIT BREAKER, 5A & Allen Bradley & 1492-SP1C050 & EA & 10 Systems & 1 & $\$ 40.00$ & $x$ & $x$ & $x$ \\
\hline CIRCUIT BREAKER, 6KA1 N POL, C.5 & Siemens & & EA & $10 \mathrm{OCR}$ & 4 & $\$ 20.48$ & $x$ & $x$ & $x$ \\
\hline CIRCUIT BREAKER, 6KA1 N POL, C1 & Siemens & & EA & 10 OCR & 4 & $\$ 20.95$ & $x$ & $x$ & $x$ \\
\hline CIRCUIT BREAKER, 6KA1 N POL, C10 & Siemens & & EA & 10 OCR & 4 & $\$ 24.88$ & $x$ & $x$ & $x$ \\
\hline CIRCUIT BREAKER, 6KA1 N POL, C3 & Siemens & & EA & 10 OCR & 4 & $\$ 20.95$ & $x$ & $x$ & $x$ \\
\hline CIRCUIT BREAKER, 6KA1 N POL, C6 & Siemens & & EA & 10 OCR & 4 & $\$ 18.34$ & $x$ & $x$ & $x$ \\
\hline COMPACT FLASH, 8 GB & ThermoFisher & GovC000119 & KIT & & 1 & $\$ 335.31$ & $x$ & $x$ & $x$ \\
\hline COMPACT FLASH, PEN DRIVE, WITH CABLE & TSA & 0463B & EA & 10 CAS & 1 & $\$ 35.00$ & $x$ & $x$ & $x$ \\
\hline CONCENTRATOR & IBase Technology & 0463A & EA & 10 CAS & 1 & $\$ 600.00$ & $x$ & $x$ & $x$ \\
\hline CONFIGURATION PLUG & Siemens & 6GK1900-0АB00 & EA & 10 Systems & 4 & $\$ 200.00$ & $x$ & $x$ & $x$ \\
\hline CONNECTOR & TSA & $2725 \mathrm{~A}$ & EA & 10 Systems & 1 & $\$ 130.00$ & $x$ & $x$ & $x$ \\
\hline CONNECTOR, BCN, MALE TO DOUBLE & HTS & 509325 & EA & 10 OCR & 3 & $\$ 15.00$ & $x$ & $x$ & $x$ \\
\hline CONNECTOR, MHV, FEMALE TO FEMALE & TSA & 2515 & EA & 10 Systems & 2 & $\$ 18.15$ & $x$ & $x$ & $x$ \\
\hline CONTROL PANEL & Aspect & 425681.005 & EA & 1 system & & $\$ 1,800.00$ & $x$ & $x$ & $x$ \\
\hline CONTROL PLATE, MASTER & TSA & 8774 & EA & & & & $x$ & $x$ & $x$ \\
\hline CONTROL PLATE, SLAVE & TSA & $8774 \mathrm{~A}$ & EA & & & & $x$ & $x$ & $x$ \\
\hline CONTROL UNIT & Aspect & SINT-GP.130 & EA & & & & $x$ & $x$ & $x$ \\
\hline CONTROLLER & Aspect & 426449.011 & EA & 1 system & & $\$ 413.00$ & $x$ & $x$ & $x$ \\
\hline CONTROLLER, MLC DROP BAR & Magnetic Autocontrol & MLC & EA & 10 Traffic Arms & 1 & $\$ 770.00$ & $x$ & $x$ & $x$ \\
\hline CONTROLLER, PROGRAMMABLE LOGIC & Automation Direct & D0-060DR-D & EA & 10 Systems & 1 & $\$ 400.00$ & $x$ & $x$ & $x$ \\
\hline CONTROLLER, SC-770 & TSA & 9411 & EA & 10 Systems & 1 & $\$ 1,312.50$ & M & $x$ & $x$ \\
\hline CONTROLLER, SCA-775, ANALOG & TSA & 9413 & EA & 10 Systems & V & $\$ 1,387.50$ & $M$ & $x$ & $x$ \\
\hline
\end{tabular}




\begin{tabular}{|c|c|c|c|c|c|c|c|c|c|}
\hline Nomenclature & Manufacturer & Part Number & UI & Basis per & $B Q$ & Cost & $\mathrm{SH}$ & $\mathrm{R}$ & $\mathrm{O}$ \\
\hline CONVERTER & Moxa & CP-132U-I & EA & & & & $x$ & $x$ & $x$ \\
\hline CONVERTER & Moxa & MOXA 132U-I & EA & & & $\$ 263.00$ & $x$ & $x$ & $x$ \\
\hline CONVERTER & TSA & 8590 & EA & & & & $x$ & $x$ & $x$ \\
\hline CONVERTER, 12 VDC TO 5 VDC & Mean Well & $7499 B$ & EA & 10 CAS & 1 & $\$ 81.00$ & $x$ & $x$ & $x$ \\
\hline CONVERTER, 48 VDC & MW & $C D(S D)-25 C-24$ & EA & 10 Systems & 1 & $\$ 920.00$ & $x$ & $x$ & $x$ \\
\hline CONVERTER, IP & Advantech & ADAM-6060-BE & EA & 10 Systems & 2 & $\$ 336.00$ & $x$ & $x$ & $x$ \\
\hline CONVERTER, MEDIA CONVERTER & Allied Telesis & AT-MC 103XL & EA & & & & $x$ & $x$ & $x$ \\
\hline CONVERTER, MEDIA CONVERTER & Moxa & IMC-101-S-SC & EA & & & & $x$ & $x$ & $x$ \\
\hline $\begin{array}{l}\text { CONVERTER, MULTI MODE, WITH SC } \\
\text { CONNECTOR }\end{array}$ & GarrettCom & & EA & Site & 1 & $\$ 750.00$ & $x$ & $x$ & $\mathrm{x}$ \\
\hline $\begin{array}{l}\text { CONVERTER, SINGLE MODE, WITH SC } \\
\text { CONNECTOR }\end{array}$ & GarrettCom & & EA & Site & 1 & $\$ 350.00$ & $x$ & $x$ & $x$ \\
\hline COUNTER, NEUTRON, SN-01 & Aspect & 412114.003 & EA & 1 system & & $\$ 1,363.00$ & $x$ & $x$ & $x$ \\
\hline COUNTER, NEUTRON, SN-02 & Aspect & 418242.002 & EA & 1 system & & $\$ 632.00$ & $x$ & $x$ & $\mathrm{x}$ \\
\hline COUPLER, BNC, FEMALE TO FEMALE & Generic Manufacturer & & EA & $10 \mathrm{OCR}$ & 5 & $\$ 3.41$ & $x$ & $x$ & $x$ \\
\hline COUPLER, SC DUPLEX, MALE TO MALE & ThermoFisher & COHD282 & KIT & & 2 & $\$ 26.14$ & $x$ & $x$ & $x$ \\
\hline CPU, X2.8 & Hewlett Packard & 397645-L22 & EA & Site & 1 & $\$ 275.85$ & $x$ & $x$ & $x$ \\
\hline $\mathrm{CPU}, \mathrm{X} 2.8$ & Hewlett Packard & 397645-B21 & EA & Site & $1-2$ & $\$ 165.12$ & $x$ & $x$ & $x$ \\
\hline CPU, X2.8 & Hewlett Packard & 397645-L22 & EA & Site & $1-2$ & $\$ 165.12$ & $x$ & $x$ & $x$ \\
\hline $\mathrm{CPU}, \mathrm{X} 2.8$ & Hewlett Packard & 397645-B21 & EA & Site & 1 & $\$ 350.00$ & $x$ & $x$ & $x$ \\
\hline CPU, X2.8 & Hewlett Packard & 397645-B21 & EA & Site & $1-2$ & $\$ 165.12$ & $x$ & $x$ & $x$ \\
\hline $\mathrm{CPU}, \mathrm{X} 2.8$ & Hewlett Packard & 397645-B21 & EA & Site & 1 & $\$ 350.00$ & $x$ & $x$ & $x$ \\
\hline $\mathrm{CPU}, \mathrm{X} 2.8$ & Hewlett Packard & 397645-L22 & EA & Site & $1-2$ & $\$ 165.12$ & $x$ & $x$ & $x$ \\
\hline CPU, XEON 5110, CPU 1 & Hewlett Packard & EX617AV & EA & Site & 1 & $\$ 104.85$ & $x$ & $x$ & $x$ \\
\hline
\end{tabular}




\begin{tabular}{|c|c|c|c|c|c|c|c|c|c|}
\hline Nomenclature & Manufacturer & Part Number & UI & Basis per & $B Q$ & Cost & $\mathrm{SH}$ & $\mathrm{R}$ & $\mathrm{O}$ \\
\hline CPU, XEON 5110, CPU 1 & Hewlett Packard & EX617AV & EA & Site & $1-2$ & $\$ 104.85$ & $x$ & $x$ & $x$ \\
\hline CPU, XEON 5110, CPU 2 & Hewlett Packard & EX618AV & EA & Site & $1-2$ & $\$ 104.85$ & $x$ & $x$ & $x$ \\
\hline CPU, XEON 5110, CPU 2 & Hewlett Packard & EX618AV & EA & Site & 1 & $\$ 104.85$ & $x$ & $x$ & $x$ \\
\hline CPU, XEON E 5504, CPU 1 & Hewlett Packard & E5504 & EA & Site & 1 & $\$ 224.00$ & $x$ & $x$ & $x$ \\
\hline CPU, XEON E 5504, CPU 2 & Hewlett Packard & E5504 & EA & Site & 1 & $\$ 235.00$ & $x$ & $x$ & $x$ \\
\hline CPU, XEON E5420, CPU 1 & Hewlett Packard & 458577-L21 & EA & Site & $1-2$ & $\$ 120.00$ & $x$ & $x$ & $x$ \\
\hline CPU, XEON E5420, CPU 1 & Hewlett Packard & GW497AV & EA & Site & 1 & $\$ 400.46$ & $x$ & $x$ & $x$ \\
\hline CPU, XEON E5420, CPU 1 & Hewlett Packard & GW497AV & EA & Site & 1 & $\$ 400.46$ & $x$ & $x$ & $x$ \\
\hline CPU, XEON E5420, CPU 1 & Hewlett Packard & GW497AV & EA & Site & $1-2$ & $\$ 400.46$ & $x$ & $x$ & $x$ \\
\hline CPU, XEON E5420, CPU 1 & Hewlett Packard & 458577-L21 & EA & Site & 1 & $\$ 120.00$ & $x$ & $x$ & $x$ \\
\hline CPU, XEON E5420, CPU 2 & Hewlett Packard & 458577-B21 & EA & Site & $1-2$ & $\$ 1,000.79$ & M & $x$ & $x$ \\
\hline CPU, XEON E5420, CPU 2 & Hewlett Packard & GW498AV & EA & Site & $1-2$ & $\$ 109.99$ & $x$ & $x$ & $x$ \\
\hline CPU, XEON E5420, CPU 2 & Hewlett Packard & GW498AV & EA & Site & 1 & $\$ 109.99$ & $x$ & $x$ & $x$ \\
\hline CPU, XEON E5420, CPU 2 & Hewlett Packard & 458577-B21 & EA & Site & 1 & $\$ 1,000.79$ & M & $x$ & $x$ \\
\hline CPU, XEON E5420, CPU 2 & Hewlett Packard & GW498AV & EA & Site & 1 & $\$ 109.99$ & $x$ & $x$ & $x$ \\
\hline CPU, XEON E5504, CPU 1 & Hewlett Packard & E5504 & EA & Site & $1-2$ & $\$ 224.00$ & $x$ & $x$ & $x$ \\
\hline CPU, XEON E5504, CPU 1 & Hewlett Packard & E5504 & EA & Site & 1 & $\$ 224.00$ & $x$ & $x$ & $x$ \\
\hline CPU, XEON E5504, CPU 2 & Hewlett Packard & E5504 & EA & Site & 1 & $\$ 235.00$ & $x$ & $x$ & $x$ \\
\hline CPU, XEON E5504, CPU 2 & Hewlett Packard & E5504 & EA & Site & $1-2$ & $\$ 235.00$ & $x$ & $x$ & $x$ \\
\hline CPU, XEON E5530, CPU 1 & Hewlett Packard & DL380G6 & EA & Site & $1-2$ & $\$ 442.00$ & $x$ & $x$ & $x$ \\
\hline CPU, XEON E5530, CPU 2 & Hewlett Packard & DL380G6 & EA & Site & 1 & $\$ 442.00$ & $x$ & $x$ & $x$ \\
\hline CPU, XEON E5530, CPU 2 & Hewlett Packard & DL380G6 & EA & Site & $1-2$ & $\$ 442.00$ & $x$ & $x$ & $x$ \\
\hline CPU, XEON, 3.6GHZ & Hewlett Packard & DY255AV & EA & Site & 1 & $\$ 833.31$ & $x$ & $x$ & $x$ \\
\hline
\end{tabular}




\begin{tabular}{|c|c|c|c|c|c|c|c|c|c|}
\hline Nomenclature & Manufacturer & Part Number & UI & Basis per & $\mathrm{BQ}$ & Cost & $\mathrm{SH}$ & $\mathrm{R}$ & $\mathrm{O}$ \\
\hline CPU, XEON, 3.6GHZ & Hewlett Packard & DY255AV & EA & Site & $1-2$ & $\$ 833.31$ & $x$ & $x$ & $x$ \\
\hline CRATE & Axis & $2911 U$ & EA & & & & $x$ & $x$ & $x$ \\
\hline CRATE & Axis & $2913 U$ & EA & & & & $x$ & $x$ & $x$ \\
\hline CRATE & ThermoFisher & & EA & & & $\$ 145.00$ & $x$ & $x$ & $x$ \\
\hline DEFROSTER, 230 VAC & Pelco & $0461 G$ & EA & 10 CAS & 1 & $\$ 72.00$ & $x$ & $x$ & $x$ \\
\hline DESICCANT & Sud Chemie & 0095 & EA & 10 Systems & V & $\$ 4.95$ & $x$ & $x$ & $x$ \\
\hline DETECTOR, GAMMA & TSA & $8200 A$ & EA & 10 Systems & V & $\$ 1,712.50$ & $M$ & $x$ & $x$ \\
\hline DETECTOR, GAMMA, 12 INCH BY 48 INCH & TSA & 8244 & EA & 10 Systems & 2 & $\$ 2,838.00$ & M & $x$ & $x$ \\
\hline DETECTOR, LOOP & APS Technology & 24-0016-014 & EA & $10 \mathrm{OCR}$ & 1 & $\$ 136.00$ & $x$ & $x$ & $x$ \\
\hline DETECTOR, LOOP, EXTENDED PRESENCE & APS Technology & $42-0002-001$ & EA & $10 \mathrm{OCR}$ & 1 & $\$ 200.00$ & $x$ & $x$ & $x$ \\
\hline DETECTOR, LOOP, MATRIX & HTS & 545010 & EA & 10 OCR & 3 & $\$ 132.00$ & $x$ & $x$ & $x$ \\
\hline DETECTOR, LOOP, SOCKET MATRIX & HTS & 545011 & EA & $10 \mathrm{OCR}$ & 3 & $\$ 36.00$ & $x$ & $x$ & $x$ \\
\hline DETECTOR, NEUTRON & Aspect & 418229.004 & EA & 1 system & & & $x$ & $x$ & $x$ \\
\hline DETECTOR, NEUTRON, BDN & Aspect & & EA & 1 system & & $\$ 4,429.00$ & $x$ & $x$ & $x$ \\
\hline DETECTOR, NEUTRON, BDN-03 & Aspect & 418229.006 & EA & 1 system & & $\$ 7,710.00$ & $x$ & $x$ & $x$ \\
\hline DETECTOR, NEUTRON, BDN-03-01 & Aspect & $418229.006-01$ & EA & 1 system & & $\$ 5,881.00$ & $x$ & $x$ & $x$ \\
\hline DETECTOR, NEUTRON, BDN-04 & Aspect & 418229.007 & EA & 1 system & & $\$ 8,321.00$ & $x$ & $x$ & $x$ \\
\hline DETECTOR, NEUTRON, BDN-16 & Aspect & 418229.024 & EA & 1 system & & $\$ 8,321.00$ & $x$ & $x$ & $x$ \\
\hline $\begin{array}{l}\text { DETECTOR, RADAR, FRONT AND REAR } \\
\text { SPEED }\end{array}$ & APS Technology & $80-0001-001$ & EA & 10 OCR & 1 & $\$ 644.00$ & $x$ & $x$ & $x$ \\
\hline DETECTOR, SCINTILLATION, BDS-G3M & Aspect & 418223.007 & EA & 1 system & & $\$ 3,121.00$ & $x$ & $x$ & $x$ \\
\hline DETECTOR, SCINTILLATION, BDS-G6-1 & Aspect & 418223.010 & EA & 1 system & & $\$ 3,931.00$ & $x$ & $x$ & $x$ \\
\hline DETECTOR, SCINTILLATION, BDS-G6-3 & Aspect & 418223.017 & EA & 1 system & & $\$ 7,311.00$ & $x$ & $x$ & $\mathrm{x}$ \\
\hline DIGITAL INPUT, IO ETHERNET & Wago & $750-432$ & EA & $10 \mathrm{OCR}$ & 4 & $\$ 8.54$ & $x$ & $x$ & $x$ \\
\hline DIGITAL INPUT, IO ETHERNET & Wago & $750-504$ & EA & 10 OCR & 2 & $\$ 34.00$ & $x$ & $x$ & $x$ \\
\hline
\end{tabular}




\begin{tabular}{|c|c|c|c|c|c|c|c|c|c|}
\hline Nomenclature & Manufacturer & Part Number & UI & Basis per & $B Q$ & Cost & $\mathrm{SH}$ & $\mathrm{R}$ & $\mathrm{O}$ \\
\hline DRIVER, SIREN & TSA & $7346 B$ & EA & 10 Systems & 1 & $\$ 27.50$ & $x$ & $x$ & $x$ \\
\hline ENCLOSURE & TSA & $6985 A$ & EA & & & & $x$ & $x$ & $x$ \\
\hline ENCLOSURE & TSA & 6985 & EA & & & & $x$ & $x$ & $x$ \\
\hline ENCLOSURE & TSA & 6987A & EA & & & & $x$ & $x$ & $x$ \\
\hline ENCLOSURE & TSA & 6987 & EA & & & & $x$ & $x$ & $x$ \\
\hline ENCLOSURE, DRIVE & Hewlett Packard & AD542B & EA & None & 0 & $\$ 130.00$ & $x$ & $x$ & $x$ \\
\hline ENCLOSURE, ENVIRONMENTAL & Pelco & & EA & 10 Systems & 1 & $\$ 100.00$ & $x$ & $x$ & $x$ \\
\hline $\begin{array}{l}\text { ENCLOSURE, ENVIRONMENTAL, HEATER } \\
\text { AND BLOWER }\end{array}$ & Pelco & $\mathrm{EH} 4718-2$ & EA & 10 Systems & 1 & $\$ 200.00$ & $x$ & $x$ & $x$ \\
\hline ENCLOSURE, MSA, 60 SINGLE & Hewlett Packard & 418408-B21 & EA & Site & $0-1$ & $\$ 4,658.00$ & M & $x$ & $x$ \\
\hline ENCLOSURE, MSA, 60 SINGLE & Hewlett Packard & 418408-B21 & EA & Site & $0-1$ & $\$ 1,450.00$ & M & $x$ & $x$ \\
\hline ENCLOSURE, MSA, 60 SINGLE & Hewlett Packard & MSA-60 & EA & Site & $0-1$ & $\$ 5,247.00$ & M & $x$ & $x$ \\
\hline ENCLOSURE, REPEATER, MACHINED & Hoffman & $7049 R$ & EA & 10 CAS & 1 & $\$ 85.00$ & $x$ & $x$ & $x$ \\
\hline END MODULE, IO ETHERNET & Wago & $750-600$ & EA & 10 OCR & 2 & $\$ 15.00$ & $x$ & $x$ & $x$ \\
\hline EXTENDER MODULE & Siemens & EM495-8 & EA & 10 Systems & 1 & $\$ 847.00$ & $x$ & $x$ & $x$ \\
\hline FAN, CIRCULATION, 115 VAC 4 INCH AXIAL & ThermoFisher & FNAC10 & KIT & & 1 & $\$ 176.52$ & $x$ & $x$ & $x$ \\
\hline FAN, RACK MOUNT, FAN ROOF & Tripp Lite & 0459F & EA & 10 CAS & 1 & $\$ 450.00$ & $x$ & $x$ & $x$ \\
\hline FIBER PONE & *** Unknown & $60-000381-02$ & EA & 10 Systems & 1 & $\$ 300.00$ & $x$ & $x$ & $x$ \\
\hline FILTER, SINGLE PHASE, EMI/RFI & ThermoFisher & LFTR001 & KIT & & 1 & $\$ 182.66$ & $x$ & $x$ & $x$ \\
\hline FIREWALL & Cisco & varies & EA & Site & 1 & $\$ 1,450.00$ & M & $x$ & $x$ \\
\hline FIREWALL & Cisco & ASA5505-50-BUN-K8 & EA & 10 Systems & 1 & $\$ 505.26$ & $x$ & $x$ & $x$ \\
\hline FIREWALL & Cisco & varies & EA & Site & 1 & $\$ 1,450.00$ & M & $x$ & $x$ \\
\hline FIREWALL, ASA 5505 & Cisco & ASA 5505 & EA & Site & 1 & $\$ 303.00$ & $x$ & $x$ & $x$ \\
\hline
\end{tabular}




\begin{tabular}{|c|c|c|c|c|c|c|c|c|c|}
\hline Nomenclature & Manufacturer & Part Number & UI & Basis per & $B Q$ & Cost & $\mathrm{SH}$ & $\mathrm{R}$ & $\mathrm{O}$ \\
\hline FLOPPY DRIVE & Hewlett Packard & ER242AV & EA & Site & $1-2$ & $\$ 28.00$ & $x$ & $x$ & $\mathrm{x}$ \\
\hline FLOPPY DRIVE & Hewlett Packard & ER242AV & EA & Site & 1 & $\$ 28.00$ & $x$ & $x$ & $x$ \\
\hline FLOPPY DRIVE & Hewlett Packard & DY290AV & EA & Site & 1 & $\$ 35.00$ & $x$ & $x$ & $x$ \\
\hline FLOPPY DRIVE & Hewlett Packard & DY290AV & EA & Site & $1-2$ & $\$ 30.00$ & $x$ & $x$ & $x$ \\
\hline FRAME GRABBER & HTS & 560217 & EA & 10 OCR & 2 & $\$ 1,120.00$ & M & $x$ & $x$ \\
\hline FUSE, $1.5 \mathrm{~A}$ & Generic Manufacturer & GGC-1/2 & EA & 10 Systems & 4 & $\$ 1.64$ & $x$ & $x$ & $x$ \\
\hline FUSE, 10A & ThermoFisher & GOVC000295 & KIT & & 2 & $\$ 21.52$ & $x$ & $x$ & $x$ \\
\hline FUSE, 12V DC 5A & Bussman & AGC-5 & EA & 10 Systems & 2 & $\$ 2.00$ & $x$ & $x$ & $x$ \\
\hline FUSE, 15A & Bussman & LP-CC-15 & EA & 10 Systems & 10 & $\$ 3.00$ & $x$ & $x$ & $x$ \\
\hline FUSE, 15A, CLASS CC & Generic Manufacturer & KTK-CC-15 & EA & 10 Systems & 1 & $\$ 20.00$ & $x$ & $x$ & $x$ \\
\hline FUSE, 5 AMP & TSA & $7170 B$ & EA & 10 Systems & & $\$ 0.90$ & $x$ & $x$ & $x$ \\
\hline FUSE, 5A & Generic Manufacturer & AGC-4 & EA & 10 Systems & 5 & $\$ 2.00$ & $x$ & $x$ & $x$ \\
\hline FUSE, 7A & Generic Manufacturer & GGC-7 & EA & 10 Systems & 5 & $\$ 3.37$ & $x$ & $x$ & $x$ \\
\hline FUSE, 8A & ThermoFisher & GOVC000296 & KIT & & 2 & $\$ 21.11$ & $x$ & $x$ & $x$ \\
\hline GRIP, CORD, $1 / 2$ & Hummel & 7305D & EA & 10 CAS & 1 & $\$ 6.50$ & $x$ & $x$ & $x$ \\
\hline GRIP, CORD, $1 / 4$ & Hummel & $7305 \mathrm{G}$ & EA & 10 CAS & 1 & $\$ 5.00$ & $x$ & $x$ & $\mathrm{x}$ \\
\hline GRIP, CORD, $3 / 4$ & Hummel & $7305 \mathrm{~F}$ & EA & 10 CAS & 1 & $\$ 10.00$ & $x$ & $x$ & $x$ \\
\hline HARNESS, WIRE, COMM POWER & ThermoFisher & ER6087C & KIT & & 1 & $\$ 528.28$ & $x$ & $x$ & $x$ \\
\hline HARNESS, WIRE, NAI DET EXT 2 & ThermoFisher & YP11702484 & KIT & & 1 & $\$ 169.51$ & $x$ & $x$ & $x$ \\
\hline HARNESS, WIRE, NAI DET EXT 3 & ThermoFisher & YP11702485 & KIT & & 1 & $\$ 169.51$ & $x$ & $x$ & $x$ \\
\hline HARNESS, WIRE, NAI DET EXT 4 & ThermoFisher & YP11702486 & KIT & & 1 & $\$ 169.51$ & $x$ & $x$ & $x$ \\
\hline HDD ARRAY & Hewlett Packard & AD554B & EA & None & 0 & $\$ 7,000.00$ & M & $x$ & $x$ \\
\hline
\end{tabular}




\begin{tabular}{|c|c|c|c|c|c|c|c|c|c|}
\hline Nomenclature & Manufacturer & Part Number & UI & Basis per & $B Q$ & Cost & $\mathrm{SH}$ & $\mathrm{R}$ & $\mathrm{O}$ \\
\hline HDD CONTROLLER CARD & Hewlett Packard & 411508-B21 & EA & Site & $1-2$ & $\$ 74.00$ & $x$ & $x$ & $x$ \\
\hline HDD CONTROLLER CARD & Hewlett Packard & 381513-B21 & EA & Site & $1-2$ & $\$ 601.19$ & $x$ & $x$ & $x$ \\
\hline HDD CONTROLLER CARD & Hewlett Packard & 337972-B21 & EA & Site & $1-2$ & $\$ 378.18$ & $x$ & $x$ & $x$ \\
\hline HDD CONTROLLER CARD & Hewlett Packard & DZ540AV & EA & Site & $1-2$ & $\$ 199.99$ & $x$ & $x$ & $x$ \\
\hline HDD CONTROLLER CARD & Hewlett Packard & 291967-B21 & EA & Site & $1-2$ & $\$ 63.00$ & $x$ & $x$ & $x$ \\
\hline HDD CONTROLLER CARD & Hewlett Packard & 291967-B21 & EA & Site & 1 & $\$ 63.00$ & $x$ & $x$ & $x$ \\
\hline HDD CONTROLLER CARD & Hewlett Packard & 291967-B21 & EA & Site & 1 & $\$ 63.00$ & $x$ & $x$ & $x$ \\
\hline HDD CONTROLLER CARD & Hewlett Packard & DZ540AV & EA & Site & 1 & $\$ 199.99$ & $x$ & $x$ & $\mathrm{x}$ \\
\hline HDD CONTROLLER CARD & Hewlett Packard & 291967-B21 & EA & Site & $1-2$ & $\$ 63.00$ & $x$ & $x$ & $\mathrm{x}$ \\
\hline $\begin{array}{l}\text { HDD CONTROLLER CARD, } 4 \text { PORT RAID, } \\
\text { SAS SATA }\end{array}$ & Hewlett Packard & & EA & Site & $1-2$ & $\$ 261.56$ & $x$ & $x$ & $x$ \\
\hline $\begin{array}{l}\text { HDD CONTROLLER CARD, } 4 \text { PORT RAID, } \\
\text { SAS SATA }\end{array}$ & Hewlett Packard & $3041 \mathrm{E}$ & EA & Site & 1 & $\$ 261.56$ & $x$ & $x$ & $x$ \\
\hline $\begin{array}{l}\text { HDD CONTROLLER CARD, } 4 \text { PORT RAID, } \\
\text { SAS SATA }\end{array}$ & Hewlett Packard & $3041 \mathrm{E}$ & EA & Site & 1 & $\$ 261.56$ & $x$ & $x$ & $x$ \\
\hline HDD CONTROLLER CARD, BBWC, E200/128 & Hewlett Packard & 411508-B21 & EA & Site & 1 & $\$ 74.00$ & $x$ & $x$ & $x$ \\
\hline HDD CONTROLLER CARD, P600/256 & Hewlett Packard & 337972-B21 & EA & Site & 1 & $\$ 378.18$ & $x$ & $x$ & $x$ \\
\hline HDD CONTROLLER CARD, P800/512 & Hewlett Packard & 381513-B21 & EA & Site & 1 & $\$ 601.19$ & $x$ & $x$ & $\mathrm{x}$ \\
\hline $\begin{array}{l}\text { HDD CONTROLLER CARD, SAS } 2 \text { PORT INT } \\
\text { AND EXT, P800 } 512 \text { BBWC }\end{array}$ & Hewlett Packard & DL380G6 & EA & Site & 1 & $\$ 999.00$ & $x$ & $x$ & $x$ \\
\hline $\begin{array}{l}\text { HDD CONTROLLER CARD, SAS } 2 \text { PORT INT } \\
\text { AND EXT, P800 } 512 \text { BBWC }\end{array}$ & Hewlett Packard & DL380G6 & EA & Site & $1-2$ & $\$ 999.00$ & $x$ & $x$ & $x$ \\
\hline HDD, DISK ARRAY, 450GB & Generic Manufacturer & & EA & 10 OCR & 2 & $\$ 285.00$ & $x$ & $x$ & $\mathrm{x}$ \\
\hline HDD, FC 10K, 300GB & Hewlett Packard & $364622-B 23$ & EA & Site & $4-6$ & $\$ 46.00$ & $x$ & $x$ & $x$ \\
\hline HDD, REMOVABLE 15K, 146GB & Generic Manufacturer & 419814-001 & EA & $10 \mathrm{OCR}$ & 2 & $\$ 256.57$ & $\mathrm{x}$ & $x$ & $\mathrm{x}$ \\
\hline HDD, SAS 10K, 146GB & Hewlett Packard & DL380G6 & EA & Site & 6 & $\$ 282.00$ & $x$ & $x$ & $x$ \\
\hline
\end{tabular}




\begin{tabular}{|c|c|c|c|c|c|c|c|c|c|}
\hline Nomenclature & Manufacturer & Part Number & UI & Basis per & $B Q$ & Cost & $\mathrm{SH}$ & $\mathrm{R}$ & $\mathrm{O}$ \\
\hline HDD, SAS 10K, 146GB & Hewlett Packard & 286716-B22 & EA & Site & 6 & $\$ 49.30$ & $x$ & $x$ & $\mathrm{x}$ \\
\hline HDD, SAS 10K, 146GB & Hewlett Packard & 286716-B22 & EA & Site & $6-12$ & $\$ 49.30$ & $x$ & $x$ & $x$ \\
\hline HDD, SAS 10K, 146GB & Hewlett Packard & 286716-B22 & EA & Site & 6 & $\$ 49.30$ & $x$ & $x$ & $x$ \\
\hline HDD, SAS 10K, 146GB & Hewlett Packard & 507125-B21 & EA & Site & 6 & $\$ 259.98$ & $x$ & $x$ & $x$ \\
\hline HDD, SAS 10K, 146GB & Hewlett Packard & DL380G6 & EA & Site & $6-12$ & $\$ 282.00$ & $x$ & $x$ & $x$ \\
\hline HDD, SAS 10K, 146GB & Hewlett Packard & 286716-B22 & EA & Site & $6-12$ & $\$ 49.30$ & $x$ & $x$ & $x$ \\
\hline HDD, SAS 10K, 146GB & Hewlett Packard & $507125-B 21$ & EA & Site & $6-12$ & $\$ 259.98$ & $x$ & $x$ & $x$ \\
\hline HDD, SAS 15K, 146GB & Hewlett Packard & & EA & Site & 1 & $\$ 200.00$ & $x$ & $x$ & $x$ \\
\hline HDD, SAS 15K, 146GB & Hewlett Packard & & EA & Site & 1 & $\$ 200.00$ & $x$ & $x$ & $x$ \\
\hline HDD, SAS 15K, 146GB & Hewlett Packard & & EA & Site & $1-2$ & $\$ 200.00$ & $x$ & $x$ & $x$ \\
\hline HDD, SAS 15K, 146GB & Hewlett Packard & & EA & Site & 1 & $\$ 200.00$ & $x$ & $x$ & $x$ \\
\hline HDD, SAS 15K, 146GB & Hewlett Packard & & EA & Site & 1 & $\$ 200.00$ & $x$ & $x$ & $x$ \\
\hline HDD, SAS 15K, 146GB & Hewlett Packard & & EA & Site & $1-2$ & $\$ 200.00$ & $x$ & $x$ & $x$ \\
\hline HDD, SAS 7.2K, 1TB & Hewlett Packard & MSA-60 & EA & Site & $1-2$ & $\$ 246.69$ & $x$ & $x$ & $x$ \\
\hline HDD, SAS 7.2K, 1TB & Hewlett Packard & 461137-B21 & EA & Site & $1-2$ & $\$ 246.69$ & $x$ & $x$ & $x$ \\
\hline HDD, SAS 7.2K, 1TB & Hewlett Packard & 461137-B21 & EA & Site & $1-2$ & $\$ 246.69$ & $x$ & $x$ & $x$ \\
\hline HDD, SAS 7.2K, 1TB & Hewlett Packard & 461137-B21 & EA & Site & $1-2$ & $\$ 246.69$ & $x$ & $x$ & $x$ \\
\hline HDD, SATA 7200, 250GB & Hewlett Packard & GM539AV & EA & Site & 1 & $\$ 317.50$ & $x$ & $x$ & $x$ \\
\hline HDD, SATA 7200, 250GB & Hewlett Packard & GM539AV & EA & Site & 1 & $\$ 317.50$ & $x$ & $x$ & $x$ \\
\hline HDD, SATA 7200, 250GB & Hewlett Packard & GM539AV & EA & Site & $1-2$ & $\$ 317.50$ & $x$ & $x$ & $\mathrm{x}$ \\
\hline HDD, SATA 7200, 250GB & Hewlett Packard & GM539AV & EA & Site & 1 & $\$ 102.75$ & $x$ & $x$ & $x$ \\
\hline HDD, SATA $7200,80 G B$ & Hewlett Packard & ER249AV & EA & Site & 1 & $\$ 159.25$ & $x$ & $x$ & $x$ \\
\hline
\end{tabular}




\begin{tabular}{|c|c|c|c|c|c|c|c|c|c|}
\hline Nomenclature & Manufacturer & Part Number & UI & Basis per & $B Q$ & Cost & $\mathrm{SH}$ & $\mathrm{R}$ & 0 \\
\hline HDD, SATA $7200,80 G B$ & Hewlett Packard & ER259AV & EA & Site & 1 & $\$ 89.00$ & $x$ & $x$ & $x$ \\
\hline HDD, SATA $7200,80 G B$ & Hewlett Packard & ER249AV & EA & Site & $1-2$ & $\$ 159.25$ & $x$ & $x$ & $x$ \\
\hline HDD, SATA 7200, 80GB & Hewlett Packard & ER259AV & EA & Site & $1-2$ & $\$ 89.00$ & $x$ & $x$ & $x$ \\
\hline HDD, SCSI 15K, 36GB & Hewlett Packard & DY277AV & EA & Site & 1 & $\$ 175.74$ & $x$ & $x$ & $x$ \\
\hline HDD, SCSI 15K, 36GB & Hewlett Packard & DY277AV & EA & Site & 1 & $\$ 175.74$ & $x$ & $x$ & $x$ \\
\hline HDD, SCSI 15K, 36GB & Hewlett Packard & DY277AV & EA & Site & $1-2$ & $\$ 175.74$ & $x$ & $x$ & $x$ \\
\hline HDD, SCSI 15K, 36GB & Hewlett Packard & DY277AV & EA & Site & $1-2$ & $\$ 175.74$ & $x$ & $x$ & $x$ \\
\hline HE3 TUBE & TSA & $6720 C$ & EA & & & & $x$ & $x$ & $x$ \\
\hline HEATER & Aspect & SINT-KYH.26.01.210 & EA & & & & $x$ & $x$ & $x$ \\
\hline HEATER STRIP & TSA & 7230 & EA & & & & $x$ & $x$ & $x$ \\
\hline HEATER, CONVECTION, 30W & B-Line & $\mathrm{EH}-30$ & EA & 10 Systems & 1 & $\$ 150.00$ & $x$ & $x$ & $x$ \\
\hline HEATER, CONVECTION, 60W & B-Line & CAW EH-60 & EA & 10 Systems & 1 & $\$ 200.00$ & $x$ & $x$ & $x$ \\
\hline HOUSING, CAMERA, 230 VAC & Pelco & $0461 \mathrm{~F}$ & EA & 10 CAS & 1 & $\$ 404.00$ & $x$ & $x$ & $x$ \\
\hline HOUSING, CAMERA, 24 VAC & Pelco & $0461 \mathrm{E}$ & EA & 10 CAS & 1 & $\$ 380.00$ & $x$ & $x$ & $x$ \\
\hline HOUSING, CAMERA, OUTDOOR & Pelco & EH4718-3 & EA & 10 Systems & 1 & $\$ 182.50$ & $x$ & $x$ & $x$ \\
\hline HUB & Aspect & Advancetech I-7513 & EA & & & $\$ 152.00$ & $x$ & $x$ & $x$ \\
\hline HUB, TEMPERATURE, E4 & ThermoFisher & MEVE325 & KIT & & 1 & $\$ 409.41$ & $x$ & $x$ & $x$ \\
\hline HUB, USB 2.0, 4 PORT & Generic Manufacturer & A1122455 & EA & 10 Systems & 1 & $\$ 50.00$ & $x$ & $x$ & $x$ \\
\hline ILLUMINATOR, IR & Extreme CCTV & 0460A & EA & 10 CAS & 1 & $\$ 732.00$ & $x$ & $x$ & $x$ \\
\hline ILLUMINATOR, IR & Raytec & RM25-30 & EA & 10 Systems & 1 & $\$ 400.00$ & $x$ & $x$ & $x$ \\
\hline ILLUMINATOR, IR & Raytec & RM100-A1-50 & EA & 10 Systems & 1 & $\$ 400.00$ & $x$ & $x$ & $x$ \\
\hline ILLUMINATOR, IR, 850NM & Bosch & EX26-LED-850M & EA & 10 Systems & 6 & $\$ 2,556.00$ & M & $x$ & $x$ \\
\hline
\end{tabular}




\begin{tabular}{|c|c|c|c|c|c|c|c|c|c|}
\hline Nomenclature & Manufacturer & Part Number & UI & Basis per & $B Q$ & Cost & $\mathrm{SH}$ & $\mathrm{R}$ & $\mathrm{O}$ \\
\hline ILLUMINATOR, LED, 50 & Raytec & & EA & 10 OCR & 3 & $\$ 452.00$ & $x$ & $x$ & $x$ \\
\hline ILLUMINATOR, SIDE FLAT ARRAY & APS Technology & $66-0003002$ & EA & $10 \mathrm{OCR}$ & 2 & $\$ 765.00$ & $x$ & $x$ & $x$ \\
\hline ILLUMINATOR, SIDE FLAT ARRAY & APS Technology & $66-0003001$ & EA & 10 OCR & 1 & $\$ 800.00$ & $x$ & $x$ & $x$ \\
\hline INDICATOR, LED & Aspect & 000002.001 & EA & 1 system & & $\$ 15.00$ & $x$ & $x$ & $x$ \\
\hline INDICATOR, LED & Aspect & 000002.002 & EA & 1 system & & $\$ 15.00$ & $x$ & $x$ & $x$ \\
\hline INDICATOR, LED & Aspect & 000002.003 & EA & 1 system & & $\$ 55.00$ & $x$ & $x$ & $x$ \\
\hline INJECTOR, POWER OVER ETHERNET & Titan Wireless & 0466B & EA & 10 CAS & 1 & $\$ 5.00$ & $x$ & $x$ & $x$ \\
\hline INTERFACE ADAPTER & Hewlett Packard & 262588-B21 & EA & Site & $1-2$ & $\$ 30.00$ & $x$ & $x$ & $x$ \\
\hline INVERTER, POWER & Mean Well & TS200-124A & EA & 10 Systems & 1 & $\$ 200.00$ & $x$ & $x$ & $x$ \\
\hline INVERTER, SINEWAVE & Exceltech & $X P-125-12$ & EA & 10 Systems & 1 & $\$ 300.00$ & $x$ & $x$ & $x$ \\
\hline IO CARD & HTS & 550117 & EA & 10 OCR & 1 & $\$ 165.00$ & $x$ & $x$ & $x$ \\
\hline IO UNIT, ETHERNET & ThermoFisher & MEVE361 & KIT & & 1 & $\$ 1,339.60$ & $M$ & $x$ & $x$ \\
\hline JUNCTION BOX & Aspect & 685624.003 & EA & 1 system & & & $x$ & $x$ & $x$ \\
\hline JUNCTION BOX & Aspect & 685624.003-02 & EA & 1 system & & & $x$ & $x$ & $x$ \\
\hline KEYBOARD, PS2 & Hewlett Packard & DZ204AV\#ABA & EA & Site & $1-2$ & $\$ 16.44$ & $x$ & $x$ & $x$ \\
\hline KEYBOARD, PS2 & Hewlett Packard & & EA & Site & $1-2$ & $\$ 30.00$ & $x$ & $x$ & $x$ \\
\hline KEYBOARD, PS2 & Hewlett Packard & GM604AV\#ABA & EA & Site & $1-2$ & $\$ 30.00$ & $x$ & $x$ & $x$ \\
\hline KEYBOARD, PS2 & Hewlett Packard & DZ204AV\#ABA & EA & Site & 1 & $\$ 16.44$ & $x$ & $x$ & $x$ \\
\hline KEYBOARD, PS2 & Hewlett Packard & & EA & Site & 1 & $\$ 30.00$ & $x$ & $x$ & $x$ \\
\hline KEYBOARD, PS2 & Hewlett Packard & DZ204AV\#ABA & EA & Site & 1 & $\$ 16.44$ & $x$ & $x$ & $x$ \\
\hline KEYBOARD, PS2 & Hewlett Packard & & EA & Site & 1 & $\$ 30.00$ & $x$ & $x$ & $x$ \\
\hline KEYBOARD, PS2 & Hewlett Packard & DZ204AV\#ABA & EA & Site & $1-2$ & $\$ 16.44$ & $x$ & $x$ & $x$ \\
\hline KEYBOARD, PS2 & Hewlett Packard & GM604AV\#ABA & EA & Site & 1 & $\$ 30.00$ & $x$ & $x$ & $x$ \\
\hline
\end{tabular}




\begin{tabular}{|c|c|c|c|c|c|c|c|c|c|}
\hline Nomenclature & Manufacturer & Part Number & UI & Basis per & $B Q$ & Cost & $\mathrm{SH}$ & $\mathrm{R}$ & $\mathrm{O}$ \\
\hline KEYBOARD, USB & Dell & $310-7949$ & EA & 10 Systems & 1 & $\$ 30.00$ & $x$ & $x$ & $\mathrm{x}$ \\
\hline KIT & SuperLogics & & KIT & Site & 1 & $\$ 3,000.00$ & M & $x$ & $x$ \\
\hline KIT, 6 FAN RACK TRAY, 220V & Hewlett Packard & 257414-B21 & KIT & 10 Systems & 1 & $\$ 786.00$ & $x$ & $x$ & $x$ \\
\hline KIT, BARRIER ARM ATTACHMENT & Magnetic Autocontrol & $1031-0180$ & KIT & 10 Traffic Arms & 4 & $\$ 40.00$ & $x$ & $x$ & $x$ \\
\hline KIT, CABLE, B020-008-17 CONSOLE & Tripp Lite & & KIT & Site & 1 & $\$ 30.00$ & $x$ & $x$ & $x$ \\
\hline KIT, CABLE, KVM & Tripp Lite & 0459K1 & KIT & 10 CAS & 1 & $\$ 22.75$ & $x$ & $x$ & $x$ \\
\hline KIT, CABLE, PLC PROGRAMMING & Automation Direct & DA-DSCBL & KIT & 10 Systems & 1 & $\$ 148.50$ & $x$ & $x$ & $x$ \\
\hline KIT, HE3 & ThermoFisher & GOVA000219 & KIT & & 1 & $\$ 129.23$ & $x$ & $x$ & $x$ \\
\hline KIT, KEYBOARD MOUSE & Microsoft & & KIT & Site & 1 & $\$ 40.00$ & $x$ & $x$ & $x$ \\
\hline KIT, LOCALIZATION & Hewlett Packard & GM506AV\#ABA & KIT & Site & 1 & $\$ 400.00$ & $x$ & $x$ & $x$ \\
\hline KIT, LOCALIZATION & Hewlett Packard & GM506AV\#ABA & KIT & Site & 1 & $\$ 400.00$ & $x$ & $x$ & $x$ \\
\hline KIT, LOCALIZATION & Hewlett Packard & GM506AV\#ABA & KIT & Site & $1-2$ & $\$ 400.00$ & $x$ & $x$ & $x$ \\
\hline KIT, LOCALIZATION & Hewlett Packard & DY250AV\#ABA & KIT & Site & 1 & $\$ 125.00$ & $x$ & $x$ & $x$ \\
\hline KIT, LOCALIZATION & Hewlett Packard & & KIT & Site & 1 & $\$ 77.00$ & $x$ & $x$ & $\mathrm{x}$ \\
\hline KIT, LOCALIZATION & Hewlett Packard & ER296AV\#ABA & KIT & Site & 1 & $\$ 76.80$ & $x$ & $x$ & $x$ \\
\hline KIT, LOCALIZATION & Hewlett Packard & & KIT & Site & 1 & $\$ 77.00$ & $x$ & $x$ & $x$ \\
\hline KIT, LOCALIZATION & Hewlett Packard & & KIT & Site & $1-2$ & $\$ 77.00$ & $x$ & $x$ & $x$ \\
\hline KIT, LOCALIZATION & Hewlett Packard & DY250AV\#ABA & KIT & Site & $1-2$ & $\$ 125.00$ & $x$ & $x$ & $x$ \\
\hline KIT, RSP & ThermoFisher & GOVA000216 & KIT & & 1 & $\$ 383.06$ & $x$ & $x$ & $x$ \\
\hline KIT, SPEED SENSOR & TSA & 6661 & KIT & 10 Systems & 1 & $\$ 1,172.00$ & M & $x$ & $x$ \\
\hline KIT, UPS & ThermoFisher & GOVA000217 & KIT & & 1 & $\$ 78.16$ & $\mathrm{H}$ & $x$ & $x$ \\
\hline $\begin{array}{l}\text { KIT, US RACKMOUNT, KEYBOARD MOUSE } \\
\text { AND MONITOR }\end{array}$ & Hewlett Packard & TFT7600 & KIT & Site & $0-1$ & $\$ 1,699.00$ & $M$ & $x$ & $x$ \\
\hline
\end{tabular}




\begin{tabular}{|c|c|c|c|c|c|c|c|c|c|}
\hline Nomenclature & Manufacturer & Part Number & UI & Basis per & $B Q$ & Cost & $\mathrm{SH}$ & $\mathrm{R}$ & $\mathrm{O}$ \\
\hline $\begin{array}{l}\text { KIT, US RACKMOUNT, KEYBOARD MOUSE } \\
\text { AND MONITOR }\end{array}$ & Hewlett Packard & AG052A & KIT & Site & $0-1$ & $\$ 799.00$ & $x$ & $x$ & $x$ \\
\hline KIT, VIS & ThermoFisher & GOVA000220 & KIT & & 1 & $\$ 47.71$ & $x$ & $x$ & $x$ \\
\hline KIT, VOIP PHONE AND LICENSE & Cisco & CP-7941G-A-CCME & KIT & 10 Systems & 1 & $\$ 325.88$ & $x$ & $x$ & $x$ \\
\hline LAMP & Phillips & F15T8/CW/ALTO & EA & 10 Systems & 1 & $\$ 20.00$ & $x$ & $x$ & $x$ \\
\hline LATCH, 3 POINT WITH KEY, ALTERED & ThermoFisher & ZP11702077 & KIT & & 1 & $\$ 352.13$ & $x$ & $x$ & $x$ \\
\hline LEAD SHIELDS & TSA & 4804 & EA & & & & $x$ & $x$ & $x$ \\
\hline LEAD TROUGH & TSA & $4804 \times 1$ & EA & & & & $x$ & $x$ & $x$ \\
\hline LENS, CAMERA, OBJECTIVE 1.6MM & Pentax & C1614A & EA & $10 \mathrm{OCR}$ & 1 & $\$ 139.00$ & $x$ & $x$ & $x$ \\
\hline LENS, CAMERA, OBJECTIVE 4.8MM & Pentax & C418DX & EA & 10 OCR & 1 & $\$ 248.07$ & $x$ & $x$ & $x$ \\
\hline LENS, HOFFMAN FIXTURE & Hoffman & 87576853 & EA & 10 Systems & 1 & $\$ 20.00$ & $x$ & $x$ & $x$ \\
\hline $\begin{array}{l}\text { LENS, IR CORRECTED AI DIRECT DRIVE, } 1 / 3 \\
\text { INCH } 2.8 \text { TO } 11 \mathrm{MM}\end{array}$ & Pelco & 13VDIR2.8-1 1 & EA & 10 Systems & 1 & $\$ 300.00$ & $x$ & $x$ & $x$ \\
\hline $\begin{array}{l}\text { LENS, IR CORRECTED AI DIRECT DRIVE, 1/3 } \\
\text { INCH } 3 \text { TO 8.5MM }\end{array}$ & Pelco & 13VDIR3-8.5 & EA & 10 Systems & 1 & $\$ 100.00$ & $x$ & $x$ & $x$ \\
\hline LENS, VIDEO & Computar & TG2Z1816AFCS & EA & & & & $x$ & $x$ & $x$ \\
\hline LICENSE, USB PLUG & HTS & 580044 & EA & $10 \mathrm{OCR}$ & 1 & $\$ 25.00$ & $x$ & $x$ & $x$ \\
\hline LIGHT POLE, WITH BUZZER & TSA & $7335 B$ & EA & & & & $x$ & $x$ & $x$ \\
\hline LIGHT, FAULT, AMBER & TSA & 7354 & EA & 10 Systems & 1 & $\$ 12.00$ & $x$ & $x$ & $x$ \\
\hline LIGHT, SEARCH, INFRARED & Aspect & PIK-42FS & EA & & & & $x$ & $x$ & $x$ \\
\hline LIGHT, STROBE, BLUE & TSA & $7346 \mathrm{~A}$ & EA & 10 Systems & 1 & $\$ 37.50$ & $x$ & $x$ & $x$ \\
\hline LIGHT, STROBE, RED & TSA & 7346 & EA & 10 Systems & 1 & $\$ 37.50$ & $x$ & $x$ & $x$ \\
\hline LIGHTNING ARRESTER & Cisco & $60-000381-01$ & EA & 10 Systems & 1 & $\$ 230.00$ & $x$ & $x$ & $x$ \\
\hline $\begin{array}{l}\text { LIMIT SWITCH, PIN HEAD WITH ROLLER } \\
\text { LEVER SPDT }\end{array}$ & Honeywell Microswitch & $\mathrm{BZ}-2 \mathrm{RL} 2-\mathrm{A} 2$ & EA & 10 Systems & 5 & $\$ 13.30$ & $x$ & $x$ & $x$ \\
\hline
\end{tabular}




\begin{tabular}{|c|c|c|c|c|c|c|c|c|c|}
\hline Nomenclature & Manufacturer & Part Number & UI & Basis per & $B Q$ & Cost & $\mathrm{SH}$ & $\mathrm{R}$ & $\mathrm{O}$ \\
\hline MACH ALUM PLATE, MASTER & TSA & $3204 C$ & EA & & & & $x$ & $x$ & $\mathrm{x}$ \\
\hline MACH ALUM PLATE, SLAVE & TSA & $3204 B$ & EA & & & & $x$ & $x$ & $\mathrm{x}$ \\
\hline MEDIA CONVERTER & *** Unknown & $59190634-7$ & EA & 10 Systems & 1 & $\$ 500.00$ & $x$ & $x$ & $x$ \\
\hline MEDIA CONVERTER & GarrettCom & CS14-1SC-i & EA & 10 Systems & 1 & $\$ 180.00$ & $x$ & $x$ & $x$ \\
\hline MEDIA CONVERTER & TSA & 8590 & EA & 10 CAS & 1 & $\$ 552.00$ & $x$ & $x$ & $x$ \\
\hline $\begin{array}{l}\text { MEDIA CONVERTER, TRANSCEIVER } \\
\text { MODULE, MULTIMODE }\end{array}$ & APS Technology & & EA & $10 \mathrm{OCR}$ & 1 & $\$ 2,000.00$ & M & $x$ & $x$ \\
\hline MEDIA MODULE & Siemens & MM491-2 & EA & 10 Systems & 2 & $\$ 649.00$ & $x$ & $x$ & $x$ \\
\hline MEDIA MODULE & Siemens & MM492-2LD & EA & 10 Systems & 2 & $\$ 1,407.00$ & M & $x$ & $x$ \\
\hline MEMORY KIT, DDR2 PC2300, 4GB 2X2 & Hewlett Packard & 343057-B21 & KIT & Site & 1 & $\$ 160.00$ & $x$ & $x$ & $x$ \\
\hline MEMORY KIT, DDR2 PC2300, 4GB 2X2 & Hewlett Packard & 343057-B21 & KIT & Site & 1 & $\$ 160.00$ & $x$ & $x$ & $x$ \\
\hline MEMORY KIT, DDR2 PC2300, 4GB 2X2 & Hewlett Packard & 343057-B21 & KIT & Site & $1-2$ & $\$ 427.06$ & $x$ & $x$ & $x$ \\
\hline MEMORY KIT, DDR2-400, 2GB 2X1 & Hewlett Packard & DY288AV & KIT & Site & 1 & $\$ 36.81$ & $x$ & $x$ & $x$ \\
\hline MEMORY KIT, DDR2-400, 2GB 2X1 & Hewlett Packard & DY288AV & KIT & Site & $1-2$ & $\$ 36.81$ & $x$ & $x$ & $x$ \\
\hline MEMORY KIT, DDR2-667, 4GB 4X1 & Hewlett Packard & GM568AV & KIT & Site & 1 & $\$ 566.31$ & $x$ & $x$ & $\mathrm{x}$ \\
\hline MEMORY KIT, DDR2-667, 4GB 4X1 & Hewlett Packard & GM568AV & KIT & Site & 1 & $\$ 566.31$ & $x$ & $x$ & $x$ \\
\hline MEMORY KIT, DDR2-667, 4GB 4X1 & Hewlett Packard & GM568AV & KIT & Site & $1-2$ & $\$ 566.31$ & $x$ & $x$ & $x$ \\
\hline MEMORY KIT, DDR3-1333, 4GB 4X1 & Hewlett Packard & & KIT & Site & 1 & $\$ 1,829.00$ & M & $x$ & $x$ \\
\hline MEMORY KIT, DDR3-1333, 4GB 4X1 & Hewlett Packard & & KIT & Site & 1 & $\$ 1,829.00$ & M & $x$ & $x$ \\
\hline MEMORY KIT, DDR3-1333, 8GB 1X8 & Hewlett Packard & DL380G6 & KIT & Site & 1 & $\$ 103.98$ & $x$ & $x$ & $x$ \\
\hline MEMORY KIT, DDR3-1333, 8GB 1X8 & Hewlett Packard & DL380G6 & KIT & Site & $1-2$ & $\$ 103.98$ & $x$ & $x$ & $x$ \\
\hline MEMORY KIT, PC2-5300, 4GB 2X2 & Hewlett Packard & 397413-B21 & KIT & Site & 1 & $\$ 65.00$ & $x$ & $x$ & $x$ \\
\hline MEMORY KIT, PC2-5300, 4GB 2X2 & Hewlett Packard & 397413-B21 & KIT & Site & $1-2$ & $\$ 65.00$ & $x$ & $x$ & $\mathrm{x}$ \\
\hline
\end{tabular}




\begin{tabular}{|c|c|c|c|c|c|c|c|c|c|}
\hline Nomenclature & Manufacturer & Part Number & UI & Basis per & $B Q$ & Cost & $\mathrm{SH}$ & $\mathrm{R}$ & $\mathrm{O}$ \\
\hline MODEM, DSL & Aspect & M30A & EA & & & & $x$ & $x$ & $x$ \\
\hline MODEM, DSL & Aspect & M1D & EA & & & & $x$ & $x$ & $x$ \\
\hline MODULE & Aspect & 426414.001 & EA & 1 system & & $\$ 494.00$ & $x$ & $x$ & $x$ \\
\hline MODULE, 4X4 & ThermoFisher & MEVE356 & KIT & & 1 & $\$ 1,528.08$ & M & $x$ & $x$ \\
\hline MODULE, COMMUNICATION, ETHERNET & Automation Direct & H0-ECOM100 & EA & 10 Systems & 1 & $\$ 200.00$ & $x$ & $x$ & $x$ \\
\hline MODULE, FIBER INSERT, SFF & ThermoFisher & MEVE355 & KIT & & 1 & $\$ 1,902.18$ & M & $x$ & $x$ \\
\hline MODULE, LANTRONIX & ThermoFisher & GovC000171 & KIT & & 1 & $\$ 1,007.91$ & $M$ & $x$ & $x$ \\
\hline MODULE, TIMING & Allen Bradley & 700-НТ3 & EA & 10 Systems & 1 & $\$ 51.00$ & $x$ & $x$ & $x$ \\
\hline MODULE, TIMING DISTRIBUTION & ThermoFisher & $5665 \mathrm{~A}$ & KIT & & 1 & $\$ 363.52$ & $x$ & $x$ & $x$ \\
\hline MONITOR, FLAT PANEL & Dell & 1908FP-BLK & EA & 10 Systems & 1 & $\$ 300.00$ & $x$ & $x$ & $x$ \\
\hline MONITOR, FLAT PANEL & Dell & 2209WA & EA & 10 Systems & 1 & $\$ 250.00$ & $x$ & $x$ & $x$ \\
\hline MONITOR, FLAT PANEL, 17 INCH & Hewlett Packard & PX849A8\#АВA & EA & Site & $1-2$ & $\$ 109.90$ & $x$ & $x$ & $x$ \\
\hline MONITOR, FLAT PANEL, 17 INCH & Hewlett Packard & L1706 & EA & Site & 1 & $\$ 109.90$ & $x$ & $x$ & $x$ \\
\hline MONITOR, FLAT PANEL, 17 INCH & Hewlett Packard & L1706 & EA & Site & 1 & $\$ 103.13$ & $x$ & $x$ & $x$ \\
\hline MONITOR, FLAT PANEL, 17 INCH & Hewlett Packard & L1706 & EA & Site & 1 & $\$ 103.13$ & $x$ & $x$ & $x$ \\
\hline MONITOR, FLAT PANEL, 17 INCH & Hewlett Packard & L1706 & EA & Site & 1 & $\$ 109.90$ & $x$ & $x$ & $x$ \\
\hline MONITOR, FLAT PANEL, 17 INCH & NEC & & EA & Site & 1 & $\$ 200.00$ & $x$ & $x$ & $x$ \\
\hline MONITOR, FLAT PANEL, 19 INCH & CTL & 0455 & EA & 10 CAS & 1 & $\$ 358.00$ & $x$ & $x$ & $x$ \\
\hline MONITOR, FLAT PANEL, 19 INCH & Hewlett Packard & L1906 & EA & Site & 1 & $\$ 109.89$ & $x$ & $x$ & $x$ \\
\hline MONITOR, FLAT PANEL, 19 INCH & Hewlett Packard & L1906 & EA & Site & 1 & $\$ 289.55$ & $x$ & $x$ & $x$ \\
\hline MONITOR, FLAT PANEL, 19 INCH & Hewlett Packard & HPL 1910 & EA & Site & 1 & $\$ 122.00$ & $x$ & $x$ & $x$ \\
\hline MONITOR, FLAT PANEL, 19 INCH & Hewlett Packard & HPL 1910 & EA & Site & 1 & $\$ 188.00$ & $x$ & $x$ & $x$ \\
\hline
\end{tabular}




\begin{tabular}{|c|c|c|c|c|c|c|c|c|c|}
\hline Nomenclature & Manufacturer & Part Number & UI & Basis per & $B Q$ & Cost & $\mathrm{SH}$ & $\mathrm{R}$ & $\mathrm{O}$ \\
\hline MONITOR, FLAT PANEL, 20 INCH & Hewlett Packard & LP2065 & EA & Site & 1 & $\$ 670.03$ & $x$ & $x$ & $x$ \\
\hline MONITOR, FLAT PANEL, 20 INCH & Hewlett Packard & EF227A4\#ABA & EA & Site & $1-2$ & $\$ 249.97$ & $x$ & $x$ & $x$ \\
\hline MONITOR, FLAT PANEL, 20 INCH & Hewlett Packard & EF227A4\#ABA & EA & Site & $1-2$ & $\$ 249.97$ & $x$ & $x$ & $x$ \\
\hline MONITOR, FLAT PANEL, 20 INCH & Hewlett Packard & EF227A4\#ABA & EA & Site & 1 & $\$ 249.97$ & $x$ & $x$ & $x$ \\
\hline MONITOR, FLAT PANEL, 20 INCH & Hewlett Packard & LP2065 & EA & Site & $1-2$ & $\$ 249.97$ & $x$ & $x$ & $x$ \\
\hline MONITOR, FLAT PANEL, 20 INCH & Hewlett Packard & LP2065 & EA & Site & 1 & $\$ 100.00$ & $x$ & $x$ & $x$ \\
\hline MONITOR, FLAT PANEL, 20 INCH & Hewlett Packard & EF227A4\#ABA & EA & Site & 1 & $\$ 249.97$ & $x$ & $x$ & $x$ \\
\hline MONITOR, TOUCH & ELO & ELO 1727 & EA & Site & $1-2$ & $\$ 446.24$ & $x$ & $x$ & $x$ \\
\hline MONITOR, TOUCH & ELO & ELO 1727 & EA & Site & $1-2$ & $\$ 446.24$ & $x$ & $x$ & $x$ \\
\hline MONITOR, VIDEO & Aspect & ВН-02-АЦ-22 & SET & & & $\$ 5,338.00$ & $x$ & $x$ & $x$ \\
\hline MONITOR, VIDEO & Aspect & 424929.010 & SET & & & & $x$ & $x$ & $x$ \\
\hline MONITOR, VIDEO & Aspect & $424929.008-02$ & SET & & & & $x$ & $x$ & $x$ \\
\hline MONITOR, VIDEO & Aspect & 424929.006-01 & SET & & & & $x$ & $x$ & $x$ \\
\hline MONITOR, VIDEO & Aspect & ВН-02-ПЦ & SET & & & $\$ 2,365.00$ & $x$ & $x$ & $x$ \\
\hline MONITOR, VIDEO & Aspect & 424929.016 & SET & & & & $x$ & $x$ & $x$ \\
\hline MONITOR, VIDEO & Aspect & 424929.020 & SET & & & & $x$ & $x$ & $x$ \\
\hline MOTOR, DRIVE, MIB-30 230V & Magnetic Autocontrol & MIB 30-C920 & EA & 10 Traffic Arms & 1 & $\$ 1,755.00$ & M & $x$ & $x$ \\
\hline MOTOR, DRIVE, MIB-40 240V & Magnetic Autocontrol & MIB 40-C920 & EA & 10 Traffic Arms & 1 & $\$ 1,855.00$ & M & $x$ & $x$ \\
\hline MOUNT, DIN RAIL & GarrettCom & 0464BD & EA & 10 CAS & 1 & $\$ 760.00$ & $x$ & $x$ & $x$ \\
\hline MOUNT, LOW PROFILE, VIBRATION & ThermoFisher & MMRU129 & KIT & & 8 & $\$ 29.00$ & $x$ & $x$ & $x$ \\
\hline MOUNT, PAN AND TILT & ThermoFisher & VEIN253 & KIT & & 1 & $\$ 100.41$ & $x$ & $x$ & $x$ \\
\hline MOUNT, RADIO, OUTDOOR & ARC Wireless & 0466M & EA & 10 CAS & 1 & $\$ 20.00$ & $x$ & $x$ & $x$ \\
\hline
\end{tabular}




\begin{tabular}{|c|c|c|c|c|c|c|c|c|c|}
\hline Nomenclature & Manufacturer & Part Number & UI & Basis per & $B Q$ & Cost & $\mathrm{SH}$ & $\mathrm{R}$ & $\mathrm{O}$ \\
\hline MOUNT, WALL & Pelco & $0461 C$ & EA & 10 CAS & 1 & $\$ 109.70$ & $x$ & $x$ & $x$ \\
\hline MOUNT, WALL & Pelco & $\mathrm{EM} 22$ & EA & 10 Systems & 1 & $\$ 100.00$ & $x$ & $x$ & $x$ \\
\hline MOUSE, PS2 & Hewlett Packard & AA360AV & EA & Site & $1-2$ & $\$ 29.95$ & $x$ & $x$ & $x$ \\
\hline MOUSE, PS2 & Hewlett Packard & GM598AV & EA & Site & 1 & $\$ 16.00$ & $x$ & $x$ & $x$ \\
\hline MOUSE, PS2 & Hewlett Packard & GM598AV & EA & Site & 1 & $\$ 16.00$ & $x$ & $x$ & $x$ \\
\hline MOUSE, PS2 & Hewlett Packard & AA360AV & EA & Site & 1 & $\$ 29.95$ & $x$ & $x$ & $x$ \\
\hline MOUSE, PS2 & Hewlett Packard & AA360AV & EA & Site & 1 & $\$ 29.95$ & $x$ & $x$ & $x$ \\
\hline MOUSE, PS2 & Hewlett Packard & GM598AV & EA & Site & $1-2$ & $\$ 16.00$ & $x$ & $x$ & $x$ \\
\hline MOUSE, PS2 & Hewlett Packard & AA360AV & EA & Site & $1-2$ & $\$ 29.95$ & $x$ & $x$ & $x$ \\
\hline MOUSE, PS2, OPTICAL & Hewlett Packard & & EA & Site & $1-2$ & $\$ 16.00$ & $x$ & $x$ & $x$ \\
\hline MOUSE, PS2, OPTICAL & Hewlett Packard & & EA & Site & 1 & $\$ 16.00$ & $x$ & $x$ & $x$ \\
\hline MOUSE, PS2, OPTICAL & Hewlett Packard & & EA & Site & 1 & $\$ 16.00$ & $x$ & $x$ & $x$ \\
\hline MOUSE, USB, OPTICAL & Dell & $310-9602$ & EA & 10 Systems & 1 & $\$ 15.00$ & $x$ & $x$ & $x$ \\
\hline NETWORK CARD, MANAGEMENT & APC & & EA & 10 Systems & 1 & $\$ 450.00$ & $x$ & $x$ & $x$ \\
\hline $\begin{array}{l}\text { NETWORK CARD, PCIE, DUAL PORT } \\
\text { GIGABIT }\end{array}$ & Hewlett Packard & 394795-B21 & EA & Site & $1-2$ & $\$ 57.00$ & $x$ & $x$ & $x$ \\
\hline $\begin{array}{l}\text { NETWORK CARD, PCIE, DUAL PORT } \\
\text { GIGABIT }\end{array}$ & Hewlett Packard & 394795-B21 & EA & Site & 1 & $\$ 57.00$ & $x$ & $x$ & $x$ \\
\hline $\begin{array}{l}\text { NETWORK CARD, PCIE, DUAL PORT } \\
\text { GIGABIT }\end{array}$ & Hewlett Packard & DL380G6 & EA & Site & 1 & $\$ 105.00$ & $x$ & $x$ & $x$ \\
\hline $\begin{array}{l}\text { NETWORK CARD, PCIE, DUAL PORT } \\
\text { GIGABIT }\end{array}$ & Hewlett Packard & DL380G6 & EA & Site & $1-2$ & $\$ 105.00$ & $x$ & $x$ & $x$ \\
\hline NETWORK CARD, PCIE, GIGABIT & Hewlett Packard & & EA & Site & 1 & $\$ 43.99$ & $x$ & $x$ & $x$ \\
\hline NETWORK CARD, PCIE, GIGABIT & Hewlett Packard & & EA & Site & $1-2$ & $\$ 43.99$ & $x$ & $x$ & $x$ \\
\hline NETWORK CARD, PCIE, GIGABIT & Hewlett Packard & & EA & Site & 1 & $\$ 43.99$ & $x$ & $x$ & $x$ \\
\hline NETWORK CARD, PCI-X, GIGABIT & Hewlett Packard & 290563-B21 & EA & Site & $1-2$ & $\$ 128.52$ & $x$ & $x$ & $x$ \\
\hline NETWORK CARD, PCI-X, GIGABIT & Hewlett Packard & 290563-B21 & EA & Site & $1-2$ & $\$ 128.52$ & $x$ & $x$ & $x$ \\
\hline
\end{tabular}




\begin{tabular}{|c|c|c|c|c|c|c|c|c|c|}
\hline Nomenclature & Manufacturer & Part Number & UI & Basis per & $B Q$ & Cost & $\mathrm{SH}$ & $\mathrm{R}$ & $\mathrm{O}$ \\
\hline NETWORK CARD, PCI-X, GIGABIT & Hewlett Packard & 290563-B21 & EA & Site & 1 & $\$ 128.52$ & $x$ & $x$ & $x$ \\
\hline NETWORK CARD, PCI-X, GIGABIT & Hewlett Packard & 290563-B21 & EA & Site & 1 & $\$ 128.52$ & $x$ & $x$ & $x$ \\
\hline NETWORK CARD, X-CH, DUAL ETHERNET & ThermoFisher & $5648 \mathrm{D}$ & KIT & & 1 & $\$ 7,259.67$ & M & $x$ & $x$ \\
\hline OPTICAL DRIVE, DVDROM CDR & Hewlett Packard & ER272AV & EA & Site & 1 & $\$ 93.06$ & $x$ & $x$ & $x$ \\
\hline OPTICAL DRIVE, DVDROM CDR & Hewlett Packard & ER272AV & EA & Site & $1-2$ & $\$ 93.06$ & $x$ & $x$ & $x$ \\
\hline OPTICAL DRIVE, DVDROM CDRW, $48 \mathrm{X}$ & Hewlett Packard & DY295AV & EA & Site & 1 & $\$ 128.62$ & $x$ & $x$ & $x$ \\
\hline OPTICAL DRIVE, DVDROM CDRW, 48X & Hewlett Packard & DY295AV & EA & Site & $1-2$ & $\$ 128.62$ & $x$ & $x$ & $x$ \\
\hline OPTICAL DRIVE, SATA, 16X DVD-ROM & Hewlett Packard & & EA & Site & $1-2$ & $\$ 48.51$ & $x$ & $x$ & $x$ \\
\hline OPTICAL DRIVE, SATA, 16X DVD-ROM & Hewlett Packard & GM577AV & EA & Site & 1 & $\$ 34.95$ & $x$ & $x$ & $x$ \\
\hline OPTICAL DRIVE, SATA, 16X DVD-ROM & Hewlett Packard & GM577AV & EA & Site & $1-2$ & $\$ 34.95$ & $x$ & $x$ & $x$ \\
\hline OPTICAL DRIVE, SATA, 16X DVD-ROM & Hewlett Packard & GM577AV & EA & Site & 1 & $\$ 34.95$ & $x$ & $x$ & $\mathrm{x}$ \\
\hline OPTICAL DRIVE, SATA, 16X DVD-ROM & Hewlett Packard & & EA & Site & 1 & $\$ 48.51$ & $x$ & $x$ & $x$ \\
\hline OPTICAL DRIVE, SATA, 16X DVD-ROM & Hewlett Packard & & EA & Site & 1 & $\$ 48.51$ & $x$ & $x$ & $x$ \\
\hline OPTICAL DRIVE, SATA, 8X 24X DVD-ROM & Hewlett Packard & 264007-B21 & EA & Site & $1-2$ & $\$ 40.99$ & $x$ & $x$ & $x$ \\
\hline OPTICAL DRIVE, SATA, 8X 24X DVD-ROM & Hewlett Packard & 264007-B21 & EA & Site & 1 & $\$ 40.99$ & $x$ & $x$ & $\mathrm{x}$ \\
\hline OPTICAL DRIVE, SATA, 8X 24X DVD-ROM & Hewlett Packard & 264007-B21 & EA & Site & 1 & $\$ 40.99$ & $x$ & $x$ & $x$ \\
\hline OPTICAL DRIVE, SATA, 8X 24X DVD-ROM & Hewlett Packard & 264007-B21 & EA & Site & $1-2$ & $\$ 40.99$ & $x$ & $x$ & $x$ \\
\hline OPTICAL DRIVE, SATA, 8X 24X DVD-ROM & Hewlett Packard & 264007-B21 & EA & Site & $1-2$ & $\$ 40.99$ & $x$ & $x$ & $x$ \\
\hline OPTICAL DRIVE, SATA, DVD-ROM CD-R & Hewlett Packard & 264007-B21 & EA & Site & 1 & $\$ 40.99$ & $x$ & $x$ & $x$ \\
\hline OPTICAL DRIVE, SATA, SLIM DVD-ROM & Hewlett Packard & DL380G6 & EA & Site & $1-2$ & $\$ 69.00$ & $x$ & $x$ & $x$ \\
\hline OPTICAL DRIVE, SATA, SLIM DVD-ROM & Hewlett Packard & DL380G6 & EA & Site & 1 & $\$ 69.00$ & $x$ & $x$ & $x$ \\
\hline PADLOCK, KEYED ALIKE & Grainger & $4 \mathrm{YH} 24$ & EA & 10 Systems & 1 & $\$ 6.20$ & $x$ & $x$ & $\mathrm{x}$ \\
\hline PANEL INSERT, PATCH, FIBER & Cables Plus & $A P-V C C 1 C B$ & EA & 10 Systems & 1 & $\$ 50.00$ & $x$ & $x$ & $x$ \\
\hline PANEL, 8X6 & Hoffman & $7055 P$ & EA & 10 CAS & 1 & $\$ 7.22$ & $x$ & $x$ & $x$ \\
\hline
\end{tabular}




\begin{tabular}{|c|c|c|c|c|c|c|c|c|c|}
\hline Nomenclature & Manufacturer & Part Number & UI & Basis per & $B Q$ & Cost & $\mathrm{SH}$ & $\mathrm{R}$ & $\mathrm{O}$ \\
\hline PATCH PANEL, 16 PORT & Tripp Lite & & EA & Site & 1 & $\$ 200.00$ & $x$ & $x$ & $x$ \\
\hline PATCH PANEL, 24 PORT & Tripp Lite & & EA & Site & 1 & $\$ 50.00$ & $x$ & $x$ & $x$ \\
\hline PIPE CLAMPS & TSA & 3470 & EA & & & & $x$ & $x$ & $x$ \\
\hline PLUG, ISOLATION & ThermoFisher & MEVE317 & KIT & & 1 & $\$ 61.31$ & $x$ & $x$ & $x$ \\
\hline PLUG, PROTECTIVE, SURGE 120 VAC & ThermoFisher & MEVE316 & KIT & & 4 & $\$ 143.10$ & $x$ & $x$ & $x$ \\
\hline PLUG, PROTECTIVE, SURGE 24 VDC & ThermoFisher & GOVC000297 & KIT & & 1 & $\$ 384.31$ & $x$ & $x$ & $x$ \\
\hline PLUG, PROTECTIVE, SURGE 5 VDC & ThermoFisher & MEVE358 & KIT & & 1 & $\$ 286.38$ & $x$ & $x$ & $x$ \\
\hline PMFX4, POTTED & TSA & $8699 P$ & EA & 10 Systems & V & $\$ 317.50$ & $x$ & $x$ & $x$ \\
\hline POLY BLOCKS & TSA & 4875 & EA & & & & $x$ & $x$ & $x$ \\
\hline POWER DISTRIBUTION, LOAD DISCONNECT & TSA & $8453 B$ & EA & 10 CAS & 1 & $\$ 165.00$ & $x$ & $x$ & $x$ \\
\hline POWER PLATE & TSA & $3204 \mathrm{E}$ & EA & & & & $x$ & $x$ & $x$ \\
\hline POWER PLATE & TSA & $8774 B$ & EA & & & & $x$ & $x$ & $x$ \\
\hline POWER STRIP, UNIVERSAL & Seven Star & 7303P & EA & 10 CAS & 1 & $\$ 13.00$ & $x$ & $x$ & $x$ \\
\hline POWER SUPPLY & Aspect & 426469.007 & EA & 1 system & & $\$ 906.00$ & $x$ & $x$ & $x$ \\
\hline POWER SUPPLY & Aspect & 426449.001 & EA & 1 system & & $\$ 1,250.00$ & $x$ & $x$ & $x$ \\
\hline POWER SUPPLY & Aspect & $426469.007-01$ & EA & 1 system & & $\$ 835.00$ & $x$ & $x$ & $x$ \\
\hline POWER SUPPLY & Aspect & 426469.006 & EA & 1 system & & $\$ 15,665.00$ & $x$ & $x$ & $x$ \\
\hline POWER SUPPLY & Aspect & $426469.007-03$ & EA & 1 system & & $\$ 1,525.00$ & $x$ & $x$ & $x$ \\
\hline POWER SUPPLY & Aspect & 426469.010 & EA & 1 system & & $\$ 1,441.00$ & $x$ & $x$ & $x$ \\
\hline POWER SUPPLY & Aspect & MZP-03 & EA & & & & $x$ & $x$ & $x$ \\
\hline POWER SUPPLY & Aspect & & EA & 1 system & & $\$ 591.00$ & $x$ & $x$ & $x$ \\
\hline POWER SUPPLY & Leviton & $12 \mathrm{~A}$ & EA & 10 Systems & 1 & $\$ 50.00$ & $x$ & $x$ & $x$ \\
\hline
\end{tabular}




\begin{tabular}{|c|c|c|c|c|c|c|c|c|c|}
\hline Nomenclature & Manufacturer & Part Number & UI & Basis per & $B Q$ & Cost & $\mathrm{SH}$ & $\mathrm{R}$ & $\mathrm{O}$ \\
\hline POWER SUPPLY & TRACOPOWER & TSL 060-112 & EA & & & & $x$ & $x$ & $x$ \\
\hline POWER SUPPLY & TRACOPOWER & TRACO & EA & & & $\$ 164.00$ & $x$ & $x$ & $x$ \\
\hline POWER SUPPLY & TRACOPOWER & TSP $140-112$ & EA & & & & $x$ & $x$ & $x$ \\
\hline POWER SUPPLY & TSA & & EA & & & & $x$ & $x$ & $x$ \\
\hline POWER SUPPLY & Videotronic & ST-NT-VZP/19 & EA & & & & $x$ & $x$ & $x$ \\
\hline POWER SUPPLY & Videotronic & Videotronic & EA & & & $\$ 385.00$ & $x$ & $x$ & $x$ \\
\hline POWER SUPPLY, 10A 24 VDC & ThermoFisher & GOVC000299 & KIT & & 1 & $\$ 565.28$ & $x$ & $x$ & $x$ \\
\hline POWER SUPPLY, 12 VDC & APS Technology & $24-0016-029$ & EA & $10 \mathrm{OCR}$ & 2 & $\$ 257.00$ & $x$ & $x$ & $x$ \\
\hline POWER SUPPLY, 12 VDC, $160 \mathrm{~W}$ & Weidmuller & 9925340012 & EA & 10 Systems & 2 & $\$ 440.00$ & $x$ & $x$ & 0 \\
\hline POWER SUPPLY, 2.5A 24V & Sitop & 6ЕP1333-2BA01 & EA & 10 Systems & 2 & $\$ 275.00$ & $x$ & $x$ & $x$ \\
\hline POWER SUPPLY, 24 VDC & APS Technology & $24-0016-030$ & EA & $10 \mathrm{OCR}$ & 1 & $\$ 305.00$ & $x$ & $x$ & $x$ \\
\hline POWER SUPPLY, 2A 12 VDC & ThermoFisher & MEVE360 & KIT & & 1 & $\$ 226.17$ & $x$ & $x$ & $x$ \\
\hline POWER SUPPLY, 2AC & Siemens & PS791 & EA & 10 Systems & 2 & $\$ 321.00$ & $x$ & $x$ & $x$ \\
\hline POWER SUPPLY, 350/370/380 & Hewlett Packard & 399771-B21 & EA & Site & $1-2$ & $\$ 125.00$ & $x$ & $x$ & $x$ \\
\hline POWER SUPPLY, 350/370/380 & Hewlett Packard & 399771-B21 & EA & Site & 1 & $\$ 110.99$ & $x$ & $x$ & $x$ \\
\hline POWER SUPPLY, 48 V AC & MW & RB17137153 & EA & 10 Systems & 1 & $\$ 6,026.00$ & M & $x$ & $x$ \\
\hline POWER SUPPLY, 48 VDC & SORENSEN & XFR60-20M13A & EA & 10 Systems & 0 & $\$ 1,735.00$ & M & $x$ & 0 \\
\hline POWER SUPPLY, 48 VDC & TDK Lambda & DPP480-48-1 & EA & 10 Systems & 0 & $\$ 300.00$ & $x$ & $x$ & $x$ \\
\hline POWER SUPPLY, 4A, 1 OUTPUT & Pelco & WCS1-4 & EA & 10 Systems & 1 & $\$ 150.00$ & $x$ & $x$ & $x$ \\
\hline POWER SUPPLY, 7.5A 13.5 VDC & Mean Well & $7499 C$ & EA & 10 CAS & 1 & $\$ 176.00$ & $x$ & $x$ & $x$ \\
\hline POWER SUPPLY, AC DC, 12V & ThermoFisher & MEVE336 & KIT & & 1 & $\$ 181.25$ & $x$ & $x$ & $x$ \\
\hline POWER SUPPLY, AC DC, 48V & ThermoFisher & MEVE337 & KIT & & 1 & $\$ 181.25$ & $x$ & $x$ & $x$ \\
\hline POWER SUPPLY, AC POWER, 460W & Hewlett Packard & DL380G6 & EA & Site & $1-2$ & $\$ 115.00$ & $x$ & $x$ & $x$ \\
\hline POWER SUPPLY, AC POWER, 460W & Hewlett Packard & DL380G6 & EA & Site & 1 & $\$ 115.00$ & $x$ & $x$ & $x$ \\
\hline
\end{tabular}




\begin{tabular}{|c|c|c|c|c|c|c|c|c|c|}
\hline Nomenclature & Manufacturer & Part Number & UI & Basis per & $B Q$ & Cost & $\mathrm{SH}$ & $\mathrm{R}$ & $\mathrm{O}$ \\
\hline $\begin{array}{l}\text { POWER SUPPLY, AC REDUNDANT, HOT } \\
\text { PLUG }\end{array}$ & Hewlett Packard & $355892-001$ & EA & Site & $1-2$ & $\$ 26.19$ & $x$ & $x$ & $x$ \\
\hline $\begin{array}{l}\text { POWER SUPPLY, AC REDUNDANT, HOT } \\
\text { PLUG }\end{array}$ & Hewlett Packard & $355892-001$ & EA & Site & 1 & $\$ 26.19$ & $x$ & $x$ & $x$ \\
\hline $\begin{array}{l}\text { POWER SUPPLY, AC REDUNDANT, HOT } \\
\text { PLUG }\end{array}$ & Hewlett Packard & $355892-001$ & EA & Site & $1-2$ & $\$ 26.19$ & $x$ & $x$ & $x$ \\
\hline $\begin{array}{l}\text { POWER SUPPLY, AC REDUNDANT, HOT } \\
\text { PLUG }\end{array}$ & Hewlett Packard & $355892-001$ & EA & Site & 1 & $\$ 26.19$ & $x$ & $x$ & $x$ \\
\hline POWER SUPPLY, CAMERA & Pelco & 0462 & EA & 10 CAS & 1 & $\$ 190.00$ & $x$ & $x$ & $x$ \\
\hline POWER SUPPLY, CAMERA & Pelco & 0462B & EA & 10 CAS & 1 & $\$ 450.00$ & $x$ & $x$ & $x$ \\
\hline POWER SUPPLY, CAMERA & Pelco & 0462A & EA & 10 CAS & 1 & $\$ 155.00$ & $x$ & $x$ & $x$ \\
\hline POWER SUPPLY, IO ETHERNET, IO & Wago & $750-612$ & EA & $10 \mathrm{OCR}$ & 2 & $\$ 23.37$ & $x$ & $x$ & $\mathrm{x}$ \\
\hline POWER SUPPLY, IO ETHERNET, IO & Wago & $787-612$ & EA & $10 \mathrm{OCR}$ & 2 & $\$ 135.00$ & $x$ & $x$ & $x$ \\
\hline POWER SUPPLY, SWITCH & GarrettCom & $0464 C$ & EA & 10 CAS & 1 & $\$ 76.00$ & $x$ & $x$ & $x$ \\
\hline PRINTER & Dell & GQ4BPG1 & EA & 10 Systems & 1 & $\$ 150.00$ & $x$ & $x$ & $x$ \\
\hline PRINTER, MULTIFUNCTION, COLOR LASER & Dell & $3115 \mathrm{CN}$ & EA & 10 Systems & 1 & $\$ 600.00$ & $x$ & $x$ & $x$ \\
\hline PRINTER, WITH SCANNER AND FAX & Hewlett Packard & & EA & Site & 1 & $\$ 150.00$ & $x$ & $x$ & $x$ \\
\hline $\begin{array}{l}\text { PROCESSOR, NEUTRON CHANNEL, BONK- } \\
03\end{array}$ & Aspect & 418244.035 & EA & 1 system & & $\$ 1,129.00$ & $x$ & $x$ & $x$ \\
\hline $\begin{array}{l}\text { PROCESSOR, NEUTRON CHANNEL, BONK- } \\
\text { 03-01 }\end{array}$ & Aspect & $418244.035-01$ & EA & 1 system & & $\$ 1,363.00$ & $x$ & $x$ & $x$ \\
\hline $\begin{array}{l}\text { PROGRAMMABLE LOGIC CONTROLLER } \\
\text { HAND HELD }\end{array}$ & Automation Direct & D2-HPP & EA & 10 Systems & 1 & $\$ 477.00$ & $x$ & $x$ & $x$ \\
\hline RACK, $25 \mathrm{U}$ & Tripp Lite & & EA & Site & 1 & $\$ 1,025.00$ & M & $x$ & $\mathrm{x}$ \\
\hline RACK, ENCLOSED, 19 INCH & Tripp Lite & 0459R & EA & 10 CAS & 1 & $\$ 1,682.00$ & M & $x$ & $x$ \\
\hline RACK, PC & Simatic & & EA & $10 \mathrm{OCR}$ & 1 & $\$ 3,345.30$ & $M$ & $x$ & $x$ \\
\hline
\end{tabular}




\begin{tabular}{|c|c|c|c|c|c|c|c|c|c|}
\hline Nomenclature & Manufacturer & Part Number & UI & Basis per & $\mathrm{BQ}$ & Cost & $\mathrm{SH}$ & $\mathrm{R}$ & $\mathrm{O}$ \\
\hline RADIO, COMPACT, $24 \mathrm{GHZ}$ & Dragonwave & CUSP24UFF & EA & 10 Systems & 1 & $\$ 6,000.00$ & $M$ & $x$ & $x$ \\
\hline RADIO, COMPACT, $24 \mathrm{GHZ}$ & Dragonwave & CUSP24UFC & EA & 10 Systems & 1 & $\$ 6,000.00$ & $M$ & $x$ & $x$ \\
\hline RADIO, COMPACT, $28 \mathrm{GHZ}$ & Dragonwave & CLHP28B2F & EA & 10 Systems & 1 & $\$ 6,000.00$ & $M$ & $x$ & $x$ \\
\hline RADIO, COMPACT, $28 \mathrm{GHZ}$ & Dragonwave & CLHP28B1C & EA & 10 Systems & 1 & $\$ 6,000.00$ & M & $x$ & $x$ \\
\hline RADIO, COMPACT, $28 \mathrm{GHZ}$ & Dragonwave & CLHP28B1F & EA & 10 Systems & 1 & $\$ 6,000.00$ & M & $x$ & $x$ \\
\hline RADIO, COMPACT, $28 \mathrm{GHZ}$ & Dragonwave & CHHP28B1C & EA & 10 Systems & 1 & $\$ 6,000.00$ & M & $x$ & $x$ \\
\hline RADIO, COMPACT, $28 \mathrm{GHZ}$ & Dragonwave & СHHP28B2C & EA & 10 Systems & 1 & $\$ 6,000.00$ & $M$ & $x$ & $x$ \\
\hline RADIO, COMPACT, $28 \mathrm{GHZ}$ & Dragonwave & CLHP28B2C & EA & 10 Systems & 1 & $\$ 6,000.00$ & $M$ & $x$ & $x$ \\
\hline RADIO, OUTDOOR & Deliberant & 0466 & EA & 10 CAS & 1 & $\$ 89.00$ & $x$ & $x$ & $x$ \\
\hline RADIO, OUTDOOR & Deliberant & 0465B & EA & 10 CAS & 1 & $\$ 330.00$ & $x$ & $x$ & $x$ \\
\hline RADIO, WIRELESS & Scaleance & OAA60 & EA & 10 Systems & 2 & $\$ 480.00$ & $x$ & $x$ & $x$ \\
\hline RADIO, WIRELESS & Scaleance & OAB60 & EA & 10 Systems & 1 & $\$ 102.99$ & $x$ & $x$ & $x$ \\
\hline RECEIVER & HTS & 547008 & EA & $10 \mathrm{OCR}$ & 1 & $\$ 216.00$ & $x$ & $x$ & $x$ \\
\hline RECEIVER, VIDEO & Videotronic & Videotronic VZP-1800 & EA & & & $\$ 450.00$ & $x$ & $x$ & $x$ \\
\hline RECEIVER, VIDEO & Videotronic & VZN-1800/19 & EA & & & & $x$ & $x$ & $x$ \\
\hline RECEIVER, VIDEO, SM RX & ICIS & $431489-002$ & EA & $10 \mathrm{OCR}$ & 1 & $\$ 513.00$ & $x$ & $x$ & $x$ \\
\hline RECEPTACLE, REMOTELY MANAGED & APS Technology & 24-0016-018 & EA & $10 \mathrm{OCR}$ & 1 & $\$ 524.00$ & $x$ & $x$ & $x$ \\
\hline REFLECTOR & Aspect & 000001.006 & EA & 1 system & & $\$ 35.00$ & $x$ & $x$ & $x$ \\
\hline RELAY, AUTOMATION DIRECT & Phoenix Contact & PLC-RSC-2300 & EA & 10 Systems & 1 & $\$ 30.00$ & $x$ & $x$ & $x$ \\
\hline RELAY, TUBE BASE, WITH PILOT LIGHT & Allen Bradley & 700-HA33Z12-4 & EA & 10 Systems & 1 & $\$ 20.00$ & $x$ & $x$ & $x$ \\
\hline REMOTE CONTROL & TSA & $6692 R$ & EA & 10 Systems & 1 & $\$ 50.00$ & $x$ & $x$ & $x$ \\
\hline REMOTE CONTROL & TSA & $6692 R$ & EA & & & & $x$ & $x$ & $x$ \\
\hline
\end{tabular}




\begin{tabular}{|c|c|c|c|c|c|c|c|c|c|}
\hline Nomenclature & Manufacturer & Part Number & UI & Basis per & $B Q$ & Cost & $\mathrm{SH}$ & $\mathrm{R}$ & $\mathrm{O}$ \\
\hline $\begin{array}{l}\text { REMOTE IO UNIT, ETHERNET, REMOTE } \\
\text { TRACK }\end{array}$ & ThermoFisher & MEVE362 & KIT & & 1 & $\$ 846.80$ & $x$ & $x$ & $x$ \\
\hline ROUTER & Cisco & varies & EA & Site & 1 & $\$ 303.00$ & $x$ & $x$ & $x$ \\
\hline ROUTER & Cisco & varies & EA & Site & 1 & $\$ 303.00$ & $x$ & $x$ & $x$ \\
\hline ROUTER & Cisco & varies & EA & Site & 1 & $\$ 303.00$ & $x$ & $x$ & $x$ \\
\hline ROUTER, 2821 & Cisco & 2821 & EA & Site & 1 & $\$ 1,357.00$ & $M$ & $x$ & $x$ \\
\hline SEAL, 2 INCH HOLE & Hoffman & 59760 & EA & Each & 1 & $\$ 50.00$ & $x$ & $x$ & $x$ \\
\hline SENSOR & TSA & $6692 \mathrm{E}$ & EA & & & & $x$ & $x$ & $x$ \\
\hline SENSOR & TSA & 6669D3 & EA & & & & $x$ & $x$ & $x$ \\
\hline SENSOR & TSA & $6666 \mathrm{D}$ & EA & & & & $x$ & $x$ & $x$ \\
\hline $\begin{array}{l}\text { SENSOR, BEAM RECEIVER AND } \\
\text { TRANSMITTER }\end{array}$ & APS Technology & 44-0001-001 & EA & $10 \mathrm{OCR}$ & 2 & $\$ 251.00$ & $x$ & $x$ & $x$ \\
\hline SENSOR, OCCUPANCY & Aspect & 000001.002 & EA & 1 system & & $\$ 389.00$ & $x$ & $x$ & $x$ \\
\hline SENSOR, OCCUPANCY & Aspect & 000001.001 & EA & 1 system & & $\$ 300.00$ & $x$ & $x$ & $x$ \\
\hline SENSOR, OCCUPANCY & Aspect & 000001.003 & EA & 1 system & & & $x$ & $x$ & $x$ \\
\hline SENSOR, OCCUPANCY & TSA & $6692 W$ & EA & 10 Systems & 1 & $\$ 320.00$ & $x$ & $x$ & $x$ \\
\hline $\begin{array}{l}\text { SENSOR, OPTICAL PAIR, PAIR SEND AND } \\
\text { RECEIVE }\end{array}$ & Sick & & EA & 10 OCR & 5 & $\$ 209.50$ & $x$ & $x$ & $x$ \\
\hline SENSOR, PHOTO ELECTRIC & Aspect & 000001.005 & EA & 1 system & & $\$ 260.00$ & $x$ & $x$ & $x$ \\
\hline SENSOR, RADAR & TSA & 6661TEU & EA & 10 Systems & 1 & $\$ 1,088.00$ & M & $x$ & $x$ \\
\hline SENSOR, TAMPER & Aspect & 000001.009 & EA & 1 system & & $\$ 41.00$ & $x$ & $x$ & $\mathrm{x}$ \\
\hline SENSOR, TEMPERATURE & ThermoFisher & MEVE326 & KIT & & 1 & $\$ 81.71$ & $x$ & $x$ & $\mathrm{x}$ \\
\hline SENSOR, TEMPERATURE, CABINET & ThermoFisher & 5659B & KIT & & 1 & $\$ 123.06$ & $x$ & $x$ & $x$ \\
\hline
\end{tabular}




\begin{tabular}{|c|c|c|c|c|c|c|c|c|c|}
\hline Nomenclature & Manufacturer & Part Number & UI & Basis per & $B Q$ & Cost & $\mathrm{SH}$ & $\mathrm{R}$ & $\mathrm{O}$ \\
\hline SENSOR, ULTRASONIC & TSA & $6661 B$ & EA & 10 Systems & 1 & $\$ 985.00$ & $x$ & $x$ & $x$ \\
\hline SENSOR, ULTRASONIC & TSA & 6666D3 & EA & 10 Systems & 1 & $\$ 632.50$ & $x$ & $x$ & $x$ \\
\hline SERVER & Aspect & DBS-01 & KIT & & & & $x$ & $x$ & $x$ \\
\hline SERVER & Aspect & 425681.031 & KIT & & & & $x$ & $x$ & $x$ \\
\hline SERVER & Cisco & & EA & Site & 1 & $\$ 700.00$ & $x$ & $x$ & $x$ \\
\hline SERVER & Cisco & & EA & Site & 1 & $\$ 300.00$ & $x$ & $x$ & $x$ \\
\hline SERVER & Hewlett Packard & $\begin{array}{l}\text { DL380R04 XEON3000 } \\
\text { 512KB }\end{array}$ & EA & & & $\$ 10,939.00$ & $x$ & $x$ & $x$ \\
\hline SERVER & Hewlett Packard & DL380G3 & EA & & & & $x$ & $x$ & $x$ \\
\hline SERVER, 1 CPU 4GB RAM, 160GB & Hewlett Packard & & EA & Site & 1 & $\$ 2,200.00$ & M & $x$ & $x$ \\
\hline SERVER, 1 CPU 4GB RAM, 250GB & Hewlett Packard & & EA & Site & 1 & $\$ 2,700.00$ & M & $x$ & $x$ \\
\hline SERVER, 1 CPU 4GB RAM, 5X300 GB & Hewlett Packard & & EA & Site & 1 & $\$ 4,200.00$ & M & $x$ & $x$ \\
\hline SERVER, 2 CPU 4GB RAM, 5X300 GB & Hewlett Packard & & EA & Site & 1 & $\$ 6,000.00$ & M & $x$ & $x$ \\
\hline SERVER, ICIS APP & ICIS & $431413-001$ & EA & $10 \mathrm{OCR}$ & 1 & $\$ 50.50$ & $x$ & $x$ & $\mathrm{x}$ \\
\hline SERVER, OCS OCR & APS Technology & 00-0001-003 & EA & $10 \mathrm{OCR}$ & 1 & $\$ 3,428.00$ & M & $x$ & $x$ \\
\hline SERVER, PENTIUM 4 & HTS & 940000 & EA & $10 \mathrm{OCR}$ & 1 & $\$ 2,500.00$ & M & $x$ & $x$ \\
\hline SERVER, PROLIANT & Hewlett Packard & DL380 G4 & EA & $10 \mathrm{OCR}$ & 1 & $\$ 342.00$ & $x$ & $x$ & $x$ \\
\hline SERVER, RACK MOUNT, 2U & Enlight & 0459A & EA & 10 CAS & 1 & $\$ 2,000.00$ & M & $x$ & $x$ \\
\hline SERVER, VIDEO & Axis & $241 Q$ & EA & & & & $x$ & $x$ & $\mathrm{x}$ \\
\hline SERVER, VIDEO & Axis & 241Q Blade & EA & & & & $x$ & $x$ & $\mathrm{x}$ \\
\hline SERVER, VIDEO & Axis & AXIS 2400 & EA & & & $\$ 1,478.00$ & $x$ & $x$ & $x$ \\
\hline SET, SWING AWAY FLANGE & Magnetic Autocontrol & $1031-5052$ & EA & 10 Traffic Arms & 2 & $\$ 330.00$ & $x$ & $x$ & $\mathrm{x}$ \\
\hline SET, TERMINAL BOARD & HTS & 580136 & EA & 10 OCR & 1 & $\$ 117.00$ & $x$ & $x$ & $x$ \\
\hline SHELF & Rittal & 7185035 & EA & 10 Systems & 1 & $\$ 300.00$ & $x$ & $x$ & $x$ \\
\hline SHELF, RACK MOUNT & Tripp Lite & 0459S & EA & 10 CAS & 1 & $\$ 146.00$ & $x$ & $x$ & $x$ \\
\hline
\end{tabular}




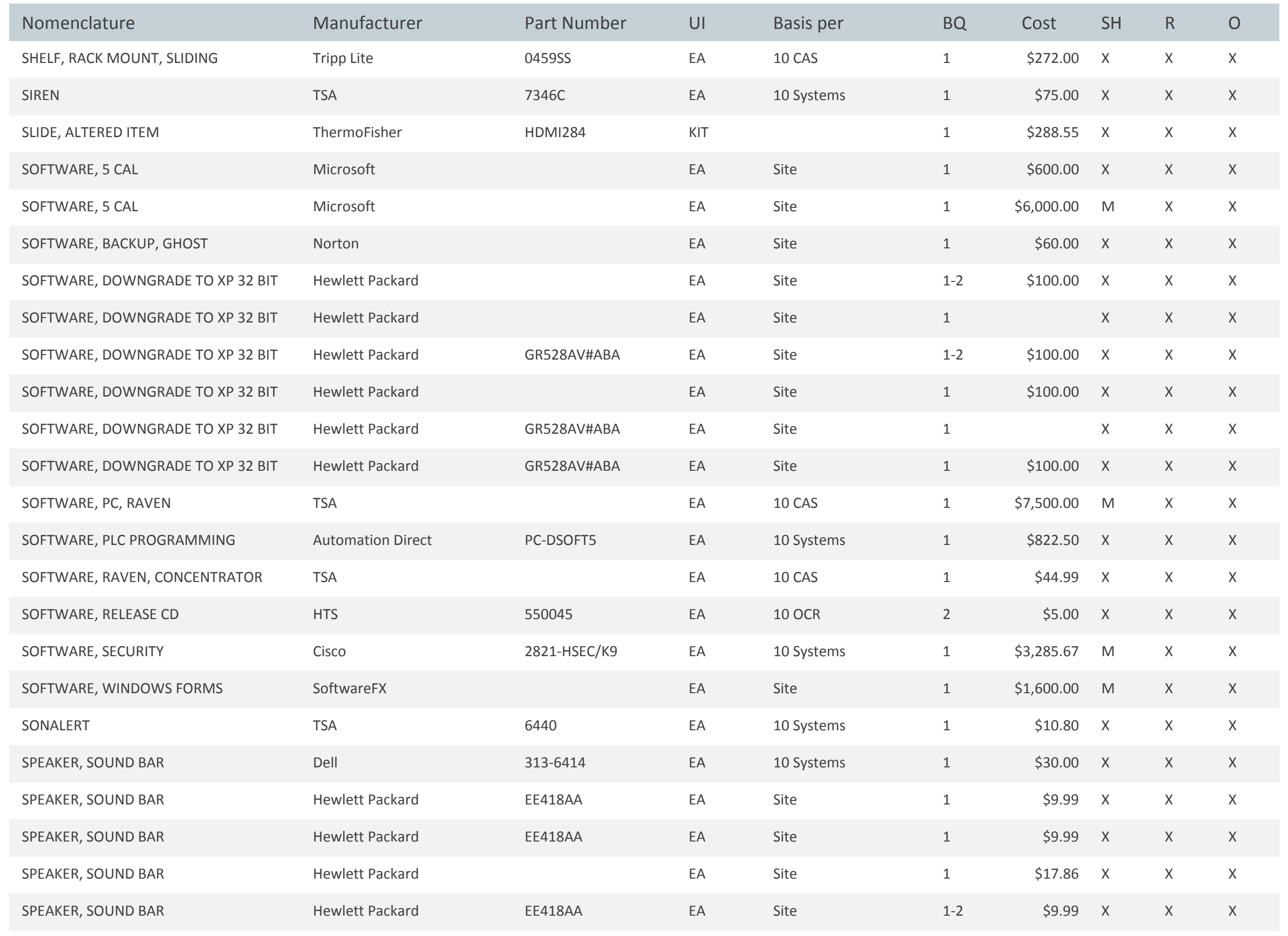




\begin{tabular}{|c|c|c|c|c|c|c|c|c|c|}
\hline Nomenclature & Manufacturer & Part Number & UI & Basis per & $B Q$ & Cost & $\mathrm{SH}$ & $\mathrm{R}$ & $\mathrm{O}$ \\
\hline SPEAKER, SOUND BAR & Hewlett Packard & & EA & Site & 1 & $\$ 17.86$ & $x$ & $x$ & $\mathrm{x}$ \\
\hline SPEAKER, SOUND BAR & Hewlett Packard & EE418AA & EA & Site & $1-2$ & $\$ 9.99$ & $x$ & $x$ & $x$ \\
\hline SPEAKER, SOUND BAR & Hewlett Packard & & EA & Site & $1-2$ & $\$ 17.86$ & $x$ & $x$ & $x$ \\
\hline $\begin{array}{l}\text { SPLITTER, BNC, FEMAL TO MALE TO } \\
\text { FEMALE }\end{array}$ & Generic Manufacturer & & EA & $10 \mathrm{OCR}$ & 5 & $\$ 6.00$ & $x$ & $x$ & $x$ \\
\hline STRAP, GROUNDING & ThermoFisher & YP11688037 & KIT & & 1 & $\$ 36.00$ & $x$ & $x$ & $x$ \\
\hline SUN SHIELD & Pelco & SS4718 & EA & 10 Systems & 1 & $\$ 200.00$ & $x$ & $x$ & $x$ \\
\hline SUN SHIELD & Pelco & $0461 \mathrm{H}$ & EA & 10 CAS & 1 & $\$ 75.00$ & $x$ & $x$ & $x$ \\
\hline SUN SHIELD & ThermoFisher & VEIN255 & KIT & & 1 & $\$ 293.26$ & $x$ & $x$ & $x$ \\
\hline SUPPLY MODULE, IO ETHERNET & Wago & $750-610$ & EA & 10 OCR & 2 & $\$ 26.60$ & $x$ & $x$ & $x$ \\
\hline SURGE PROTECTOR & Deliberant & 0466A & EA & 10 CAS & 1 & $\$ 30.00$ & $x$ & $x$ & $x$ \\
\hline SURGE SUPPRESSOR & Zerosurge & TVS230LC10 & EA & 10 Systems & 1 & $\$ 300.00$ & $x$ & $x$ & 0 \\
\hline SWITCH & Cisco & $2950-24$ & EA & & & & $x$ & $x$ & $x$ \\
\hline $\begin{array}{l}\text { SWITCH TRANSCEIVER, GIGABIT } \\
\text { EHTERNET, SFP }\end{array}$ & ICIS & $421063-001$ & EA & 10 OCR & 2 & $\$ 67.24$ & $x$ & $x$ & $x$ \\
\hline SWITCH, 1000 BASE T & Cisco & GLC-T & EA & 10 Systems & 1 & $\$ 236.19$ & $x$ & $x$ & $x$ \\
\hline SWITCH, 16 PORT, ETHERNET & Netgear & 0464G & EA & 10 CAS & 1 & $\$ 61.45$ & $x$ & $x$ & $x$ \\
\hline SWITCH, 24 PORT & Cisco & varies & EA & Site & $1-2$ & $\$ 1,464.00$ & $M$ & $x$ & $x$ \\
\hline SWITCH, 24 PORT & Cisco & varies & EA & Site & 1 & $\$ 1,464.00$ & M & $x$ & $x$ \\
\hline SWITCH, 24 PORT & Cisco & varies & EA & Site & $1-2$ & $\$ 1,464.00$ & $M$ & $x$ & $x$ \\
\hline SWITCH, 24 PORT & Cisco & varies & EA & Site & 1 & $\$ 1,464.00$ & $M$ & $x$ & $x$ \\
\hline SWITCH, 24 PORT & Cisco & varies & EA & Site & $1-2$ & $\$ 1,464.00$ & M & $x$ & $x$ \\
\hline SWITCH, 24 PORT & Cisco & $2960-24$ & EA & Site & $1-2$ & $\$ 356.00$ & $x$ & $x$ & $x$ \\
\hline
\end{tabular}




\begin{tabular}{|c|c|c|c|c|c|c|c|c|c|}
\hline Nomenclature & Manufacturer & Part Number & UI & Basis per & $\mathrm{BQ}$ & Cost & $\mathrm{SH}$ & $\mathrm{R}$ & $\mathrm{O}$ \\
\hline SWITCH, 24 PORT & Cisco & varies & EA & Site & 1 & $\$ 356.00$ & $x$ & $x$ & $x$ \\
\hline SWITCH, 24 PORT & Cisco & $2960-24$ & EA & Site & 1 & $\$ 3,795.00$ & $M$ & $x$ & $x$ \\
\hline SWITCH, 24 PORT & Cisco & varies & EA & Site & $1-2$ & $\$ 356.00$ & $x$ & $x$ & $x$ \\
\hline SWITCH, 24 PORT & Cisco & varies & EA & Site & 1 & $\$ 378.16$ & $x$ & $x$ & $x$ \\
\hline SWITCH, 24 PORT & Cisco & varies & EA & Site & 1 & $\$ 356.00$ & $x$ & $x$ & $x$ \\
\hline SWITCH, 24 PORT & Dell & & EA & 10 Systems & 1 & $\$ 140.00$ & $x$ & $x$ & $x$ \\
\hline SWITCH, 24 PORT & GarrettCom & 0464E & EA & 10 CAS & 1 & $\$ 1,700.00$ & $M$ & $x$ & $x$ \\
\hline SWITCH, 24 PORT, GIGABIT & APS Technology & $56-0015-002$ & EA & $10 \mathrm{OCR}$ & 1 & $\$ 845.00$ & $x$ & $x$ & $x$ \\
\hline SWITCH, 48 PORT & Cisco & varies & EA & Site & $1-2$ & $\$ 1,357.00$ & $M$ & $x$ & $x$ \\
\hline SWITCH, 48 PORT & Cisco & $3750-48$ & EA & Site & $1-2$ & $\$ 1,464.00$ & M & $x$ & $x$ \\
\hline SWITCH, 48 PORT & Cisco & varies & EA & Site & $1-2$ & $\$ 1,357.00$ & $M$ & $x$ & $x$ \\
\hline SWITCH, 48 PORT & Cisco & varies & EA & Site & 1 & $\$ 1,464.00$ & M & $x$ & $x$ \\
\hline SWITCH, 48 PORT & Cisco & varies & EA & Site & 1 & $\$ 378.16$ & $x$ & $x$ & $x$ \\
\hline SWITCH, 48 PORT & Cisco & varies & EA & Site & 1 & $\$ 1,357.00$ & M & $x$ & $x$ \\
\hline SWITCH, 48 PORT & Cisco & $3750-48$ & EA & Site & 1 & $\$ 1,464.00$ & M & $x$ & $x$ \\
\hline SWITCH, 48 PORT & Cisco & varies & EA & Site & 1 & $\$ 1,357.00$ & M & $x$ & $x$ \\
\hline SWITCH, 48 PORT & Cisco & varies & EA & Site & $1-2$ & $\$ 1,357.00$ & M & $x$ & $x$ \\
\hline SWITCH, 6 PORT, 12 VDC & GarrettCom & 0464B & EA & 10 CAS & 1 & $\$ 340.00$ & $x$ & $x$ & $x$ \\
\hline SWITCH, 8 PORT KVM & Tripp Lite & & EA & Site & 1 & $\$ 1,300.00$ & M & $x$ & $x$ \\
\hline SWITCH, 8 PORT, GBE WEB MANAGED & Dell & FPX9FH1 & EA & 10 Systems & 1 & $\$ 174.00$ & $x$ & $x$ & $x$ \\
\hline SWITCH, CAT5 KVM, 0X1X8 & Hewlett Packard & & EA & Site & $0-1$ & $\$ 353.00$ & $x$ & $x$ & $x$ \\
\hline SWITCH, CAT5 KVM, 0X2X16 & Hewlett Packard & 336045-B21 & EA & Site & $0-1$ & $\$ 241.84$ & $x$ & $x$ & $x$ \\
\hline
\end{tabular}




\begin{tabular}{|c|c|c|c|c|c|c|c|c|c|}
\hline Nomenclature & Manufacturer & Part Number & UI & Basis per & $B Q$ & Cost & $\mathrm{SH}$ & $\mathrm{R}$ & $\mathrm{O}$ \\
\hline SWITCH, CATALYST & Cisco & WS-C3750-24FS-E & EA & 10 Systems & 1 & $\$ 6,454.74$ & $M$ & $x$ & $x$ \\
\hline SWITCH, CATALYST & Cisco & WS-C3750G-12S-E & EA & 10 Systems & & & $x$ & $x$ & $x$ \\
\hline SWITCH, CATALYST & Cisco & WS-C3750G-24T-E & EA & 10 Systems & 1 & $\$ 2,700.00$ & $M$ & $x$ & $x$ \\
\hline SWITCH, CATALYST & Cisco & WS-C3750G-24PS-E & EA & 10 Systems & 1 & $\$ 6,992.05$ & M & $x$ & $x$ \\
\hline SWITCH, CATALYST & Cisco & WS-C2955T-12 & EA & 10 Systems & 1 & $\$ 1,800.00$ & M & $x$ & $x$ \\
\hline SWITCH, CONVERTER, MULTIMODE & GarrettCom & CS14H-SC-12VDC & EA & 10 Systems & 1 & $\$ 450.00$ & $x$ & $x$ & $x$ \\
\hline SWITCH, CONVERTER, SINGLE MODE & GarrettCom & CS14H-SSC-12VDC & EA & 10 Systems & 1 & $\$ 450.00$ & $x$ & $x$ & $x$ \\
\hline SWITCH, ETHERNET & Advantech & ADAM-6520 & EA & & & & $x$ & $x$ & $x$ \\
\hline SWITCH, ETHERNET & Advantech & ADAM 6520 & EA & & & $\$ 210.00$ & $x$ & $x$ & $x$ \\
\hline SWITCH, ETHERNET & Advantech & EKI-2528 & EA & & & & $x$ & $x$ & $x$ \\
\hline SWITCH, ETHERNET & Cisco & 2960-24TT-L & EA & & & & $x$ & $x$ & $x$ \\
\hline SWITCH, ETHERNET & Cisco & Cisco Catalyst 2950-24 & EA & & & $\$ 1,157.00$ & $x$ & $x$ & $x$ \\
\hline SWITCH, ETHERNET & GarrettCom & ES42H-2SSC-Hi & EA & 10 Systems & 1 & $\$ 780.00$ & $x$ & $x$ & $x$ \\
\hline SWITCH, ETHERNET & Moxa & MOXA EDS-305-S-SC-T & EA & & & & $x$ & $x$ & $x$ \\
\hline SWITCH, ETHERNET, HARDENED & Advantech & EKI-7758F & EA & 10 Systems & 1 & $\$ 12,632.33$ & M & $x$ & $x$ \\
\hline SWITCH, ETHERNET, UNMANAGED & ThermoFisher & MEVE368 & KIT & & 1 & $\$ 983.30$ & $x$ & $x$ & $x$ \\
\hline SWITCH, KVM & Tripp Lite & 0459K & EA & 10 CAS & 1 & $\$ 500.00$ & $x$ & $x$ & $x$ \\
\hline SWITCH, MEDIA CONVERTER & GarrettCom & CSG14H-SX-HD & EA & 10 Systems & 1 & $\$ 780.00$ & $x$ & $x$ & $x$ \\
\hline SWITCH, RACK MOUNT, 24 PORT & Cisco & & EA & Site & 1 & $\$ 1,140.00$ & M & $x$ & $x$ \\
\hline SWITCH, RACK MOUNT, 48 PORT & Cisco & & EA & Site & 1 & $\$ 2,100.00$ & M & $x$ & $x$ \\
\hline SWITCH, SAN, 4/8 BASE & Hewlett Packard & A7984A & EA & None & 0 & $\$ 555.00$ & $x$ & $x$ & $x$ \\
\hline SWITCH, SCALEANCE & Scaleance & X310 FE & EA & 10 Systems & 3 & $\$ 23.80$ & $x$ & $x$ & $x$ \\
\hline
\end{tabular}




\begin{tabular}{|c|c|c|c|c|c|c|c|c|c|}
\hline Nomenclature & Manufacturer & Part Number & UI & Basis per & $B Q$ & Cost & $\mathrm{SH}$ & $\mathrm{R}$ & $\mathrm{O}$ \\
\hline SWITCH, SCALEANCE & Scaleance & X101-1 & EA & 10 Systems & 3 & $\$ 497.62$ & $x$ & $x$ & $x$ \\
\hline SWITCH, SCALEANCE & Scaleance & X308 2LD & EA & 10 Systems & 2 & $\$ 1,686.00$ & M & $x$ & $x$ \\
\hline SWITCH, SCALEANCE & Scaleance & $x 408-2$ & EA & 10 Systems & 1 & $\$ 5,238.09$ & M & $x$ & $x$ \\
\hline SWITCH, SCALEANCE & Scaleance & X414 3E & EA & 10 Systems & 1 & $\$ 775.00$ & $x$ & $x$ & $x$ \\
\hline SWITCH, SERIAL TO ETHERNET & ThermoFisher & MEVE369 & KIT & & 1 & $\$ 587.11$ & $x$ & $x$ & $x$ \\
\hline SWITCH, TAMPER, MAGNETIC & George Fix Industries & 50F-12WG & EA & 10 Systems & 1 & $\$ 5.00$ & $x$ & $x$ & $x$ \\
\hline SWITCH, TAMPER, MAGNETIC & TSA & 7818 & EA & 10 Systems & V & $\$ 9.00$ & $x$ & $x$ & $x$ \\
\hline SYNCHRONIZATION GENERATOR & HTS & 910373 & EA & $10 \mathrm{OCR}$ & 1 & $\$ 635.00$ & $x$ & $x$ & $x$ \\
\hline TAPE DRIVE, MSL & Hewlett Packard & BL531A & EA & Site & $0-1$ & $\$ 4,925.00$ & M & $x$ & $x$ \\
\hline TAPE DRIVE, MSL & Hewlett Packard & BL531A & EA & Site & $0-1$ & $\$ 4,925.00$ & M & $x$ & $x$ \\
\hline TAPE DRIVE, MSL & Hewlett Packard & MSL 2024 & EA & Site & $0-1$ & $\$ 1,614.00$ & $M$ & $x$ & $x$ \\
\hline TAPE DRIVE, MSL & Hewlett Packard & BL531A & EA & Site & $0-1$ & $\$ 4,925.00$ & M & $x$ & $x$ \\
\hline TELEPHONY, VOIP & Cisco & SPA901 & EA & 10 Systems & 1 & $\$ 100.00$ & $x$ & $x$ & $x$ \\
\hline TERMINAL & TSA & $2533 \mathrm{~F}$ & EA & & & & $x$ & $x$ & $x$ \\
\hline TERMINAL & TSA & $2533 C$ & EA & & & & $x$ & $x$ & $x$ \\
\hline TERMINAL & TSA & 2532 & EA & & & & $x$ & $x$ & $x$ \\
\hline TERMINAL & TSA & $2533 D$ & EA & & & & $x$ & $x$ & $x$ \\
\hline TERMINAL & TSA & $2533 \mathrm{~A}$ & EA & & & & $x$ & $x$ & $\mathrm{x}$ \\
\hline TERMINAL, FUSIBLE, WITH LED & Phoenix Contact & UT 6-HESI & EA & 10 Systems & 1 & $\$ 20.00$ & $x$ & $x$ & $x$ \\
\hline TEST BENCH, 28GHZ & Dragonwave & $60-000327-08-01$ & EA & 10 Systems & 1 & $\$ 100.00$ & $x$ & $x$ & $x$ \\
\hline THERMO-HOUSING & Aspect & GlobalProof CKS-600 & EA & & & & $x$ & $x$ & $x$ \\
\hline THERMO-HOUSING & Aspect & SVS-E210 & EA & & & & $x$ & $x$ & $x$ \\
\hline
\end{tabular}




\begin{tabular}{|c|c|c|c|c|c|c|c|c|c|}
\hline Nomenclature & Manufacturer & Part Number & UI & Basis per & $B Q$ & Cost & $\mathrm{SH}$ & $\mathrm{R}$ & $\mathrm{O}$ \\
\hline TIES, CABLE & TSA & $3450 \mathrm{C}$ & EA & & & & $x$ & $x$ & $x$ \\
\hline TRANCEIVER, FIBER CONVERTER, LXLH & Cisco & GLC-LH-SM & EA & 10 OCR & 3 & $\$ 421.74$ & $x$ & $x$ & $x$ \\
\hline TRANCEIVER, LC CONNECTOR SX & Cisco & GLC-SX-MM & EA & 10 Systems & 1 & $\$ 298.97$ & $x$ & $x$ & $x$ \\
\hline TRANSMITTER & HTS & 547007 & EA & 10 OCR & 1 & $\$ 222.00$ & $x$ & $x$ & $x$ \\
\hline TRANSMITTER, VIDEO & Videotec & TWM2 & EA & & & & $x$ & $x$ & $x$ \\
\hline TRANSMITTER, VIDEO & Videotronic & VSZ-BNC & EA & & & & $x$ & $x$ & $x$ \\
\hline TRIM, BLACK BULB, 7/16 O.D. & ThermoFisher & HDTA201 & KIT & & 24 & $\$ 95.77$ & $x$ & $x$ & $x$ \\
\hline UPS & APC & SC620I & EA & & & & $x$ & $x$ & $x$ \\
\hline UPS & APC & $\begin{array}{l}\text { Smart UPS APC } \\
1000 \times \mathrm{LI}\end{array}$ & EA & & & $\$ 1,560.00$ & $x$ & $x$ & $x$ \\
\hline UPS & Aspect & SU2200RMXLINET & EA & & & & $x$ & $x$ & $x$ \\
\hline UPS & Aspect & & EA & 1 system & & $\$ 1,257.00$ & $x$ & $x$ & $x$ \\
\hline UPS & Aspect & $\begin{array}{l}\text { Smart UPS } \\
\text { SU2000RMXLINE }\end{array}$ & EA & & & $\$ 2,080.00$ & $x$ & $x$ & $x$ \\
\hline UPS & Aspect & 436234.043 & EA & 1 system & & $\$ 1,265.00$ & $x$ & $x$ & $x$ \\
\hline UPS & Aspect & 436234.029 & EA & 1 system & & $\$ 1,257.00$ & $x$ & $x$ & $x$ \\
\hline UPS & Mean Well & DR-UPS-40 & EA & 10 Systems & 1 & $\$ 100.00$ & $\mathrm{H}$ & $x$ & $x$ \\
\hline UPS, 1400VA & APC & SUI400RMXLIB3U & EA & 10 Systems & 3 & $\$ 2,185.26$ & $\mathrm{MH}$ & $x$ & $x$ \\
\hline UPS, 1400VA & APC & SU1400RMXLB3U & EA & 10 Systems & 1 & $\$ 2,185.00$ & $\mathrm{MH}$ & $x$ & $\mathrm{O}$ \\
\hline UPS, 1500 VA $120 \mathrm{~V}$ & APC & & EA & 10 Systems & 1 & $\$ 300.00$ & $\mathrm{H}$ & $x$ & $x$ \\
\hline UPS, 1500VA & APC & $\begin{array}{l}1500 \text { (exact model } \\
\text { country/region } \\
\text { specific) }\end{array}$ & EA & Site & 1 & $\$ 420.00$ & $\mathrm{H}$ & $x$ & $x$ \\
\hline UPS, 1500VA & APC & $\begin{array}{l}1500 \text { (exact model } \\
\text { country/region } \\
\text { specific) }\end{array}$ & EA & Site & 1 & $\$ 700.00$ & $\mathrm{H}$ & $x$ & $x$ \\
\hline
\end{tabular}




\begin{tabular}{|c|c|c|c|c|c|c|c|c|c|}
\hline Nomenclature & Manufacturer & Part Number & UI & Basis per & $B Q$ & Cost & $\mathrm{SH}$ & $\mathrm{R}$ & $\mathrm{O}$ \\
\hline UPS, 1500VA & APC & $\begin{array}{l}1500 \text { (exact model } \\
\text { country/region } \\
\text { specific) }\end{array}$ & EA & Site & $1-2$ & $\$ 150.00$ & $\mathrm{H}$ & $x$ & $x$ \\
\hline UPS, 1500VA & APC & varies & EA & Site & 1 & $\$ 150.00$ & $\mathrm{H}$ & $x$ & $x$ \\
\hline UPS, 1500VA & APC & varies & EA & Site & $1-2$ & $\$ 150.00$ & $\mathrm{H}$ & $x$ & $x$ \\
\hline UPS, 1500VA & APC & varies & EA & Site & $1-2$ & $\$ 150.00$ & $\mathrm{H}$ & $x$ & $x$ \\
\hline UPS, 1500VA & APC & varies & EA & Site & $1-2$ & $\$ 150.00$ & $\mathrm{H}$ & $x$ & $x$ \\
\hline UPS, 1500VA & APC & varies & EA & Site & 1 & $\$ 149.92$ & $\mathrm{H}$ & $x$ & $x$ \\
\hline UPS, 1500VA & APC & varies & EA & Site & 1 & $\$ 150.00$ & $\mathrm{H}$ & $x$ & $x$ \\
\hline UPS, 1500VA & APC & BR15001 & EA & 10 Systems & 1 & $\$ 235.79$ & $\mathrm{H}$ & $x$ & $x$ \\
\hline UPS, 1500VA & APC & varies & EA & Site & 1 & $\$ 149.92$ & $\mathrm{H}$ & $x$ & $x$ \\
\hline UPS, 1500VA & Tripp Lite & & EA & Site & 1 & $\$ 600.00$ & $\mathrm{H}$ & $x$ & $x$ \\
\hline UPS, 1500VA & TSA & 0476A & EA & 10 CAS & 1 & $\$ 925.25$ & $\mathrm{H}$ & $x$ & $x$ \\
\hline UPS, 2300 VA & Minute Man & 0475 & EA & 10 CAS & 1 & $\$ 1,000.00$ & $\mathrm{MH}$ & $x$ & $x$ \\
\hline UPS, 3000VA & APC & varies & EA & Site & 1 & $\$ 925.00$ & $\mathrm{H}$ & $x$ & $x$ \\
\hline UPS, 3000VA & APC & varies & EA & Site & 1 & $\$ 925.00$ & $\mathrm{H}$ & $x$ & $x$ \\
\hline UPS, 3000VA & APC & varies & EA & Site & 1 & $\$ 925.00$ & $\mathrm{H}$ & $x$ & $x$ \\
\hline UPS, 3000VA & APC & varies & EA & Site & 1 & $\$ 925.00$ & $\mathrm{H}$ & $x$ & $x$ \\
\hline UPS, 3000VA & APC & varies & EA & Site & 1 & $\$ 925.00$ & $\mathrm{H}$ & $x$ & $x$ \\
\hline UPS, 3000VA & APC & $\begin{array}{l}3000 \text { (exact model } \\
\text { country/region } \\
\text { specific) }\end{array}$ & EA & Site & 1 & $\$ 925.00$ & $\mathrm{H}$ & $x$ & $x$ \\
\hline UPS, 3000VA & APC & $\begin{array}{l}3000 \text { (exact model } \\
\text { country/region } \\
\text { specific) }\end{array}$ & EA & Site & 1 & $\$ 925.00$ & $\mathrm{H}$ & $x$ & $x$ \\
\hline UPS, 3000VA & APC & varies & EA & Site & 1 & $\$ 925.00$ & $\mathrm{H}$ & $x$ & $x$ \\
\hline UPS, 3000VA, RACK & AEG & & EA & 10 OCR & 2 & $\$ 1,126.49$ & $\mathrm{MH}$ & $x$ & $x$ \\
\hline
\end{tabular}




\begin{tabular}{|c|c|c|c|c|c|c|c|c|c|}
\hline Nomenclature & Manufacturer & Part Number & UI & Basis per & $B Q$ & Cost & $\mathrm{SH}$ & $\mathrm{R}$ & $\mathrm{O}$ \\
\hline UPS, 5000VA & APC & varies & EA & Site & 1 & $\$ 3,001.00$ & $\mathrm{MH}$ & $x$ & $x$ \\
\hline UPS, 5000VA & APC & $\begin{array}{l}5000 \text { (exact model } \\
\text { country/region } \\
\text { specific) }\end{array}$ & EA & Site & 1 & $\$ 3,001.00$ & $\mathrm{MH}$ & $x$ & $x$ \\
\hline UPS, 5000VA & APC & varies & EA & Site & 1 & $\$ 3,001.00$ & $\mathrm{MH}$ & $x$ & $x$ \\
\hline UPS, 5000VA & APC & varies & EA & Site & 1 & $\$ 3,001.00$ & $\mathrm{MH}$ & $x$ & $x$ \\
\hline UPS, 6000VA & APC & SURT6000XLI & EA & 10 Systems & 1 & $\$ 3,036.84$ & $\mathrm{MH}$ & $x$ & $x$ \\
\hline UPS, 750 VA & Tripp Lite & 0476 & EA & 10 CAS & 1 & $\$ 340.00$ & $\mathrm{H}$ & $x$ & $x$ \\
\hline UPS, 750VA & Tripp Lite & & EA & Site & 1 & $\$ 130.00$ & $\mathrm{H}$ & $x$ & $x$ \\
\hline UPS, DIN RAIL MOUNTABLE & SOLA & SDU 850 & EA & 10 Systems & 1 & $\$ 500.00$ & $\mathrm{H}$ & $x$ & $x$ \\
\hline UTILITY BOX, 8X6X4 & Hoffman & 7055B & EA & 10 CAS & 1 & $\$ 21.76$ & $x$ & $x$ & $x$ \\
\hline VD580, POTTED & TSA & 8870AP & EA & 10 Systems & V & $\$ 142.50$ & $x$ & $x$ & $x$ \\
\hline VIDEO CARD, NVIDIA QUADRO, 128MB & Hewlett Packard & RD064AV & EA & Site & $1-2$ & $\$ 54.95$ & $x$ & $x$ & $\mathrm{x}$ \\
\hline VIDEO CARD, NVIDIA QUADRO, 128MB & Hewlett Packard & EE058AV & EA & Site & $1-2$ & $\$ 147.41$ & $x$ & $x$ & $x$ \\
\hline VIDEO CARD, NVIDIA QUADRO, 128MB & Hewlett Packard & RD064AV & EA & Site & 1 & $\$ 54.95$ & $x$ & $x$ & $x$ \\
\hline VIDEO CARD, NVIDIA QUADRO, 128MB & Hewlett Packard & EE058AV & EA & Site & 1 & $\$ 147.41$ & $x$ & $x$ & $x$ \\
\hline VIDEO CARD, NVIDIA QUADRO, 256MB & Hewlett Packard & NVS295 & EA & Site & 1 & $\$ 132.00$ & $x$ & $x$ & $\mathrm{x}$ \\
\hline VIDEO CARD, NVIDIA QUADRO, 256MB & Hewlett Packard & GN493AV & EA & Site & 1 & $\$ 49.00$ & $x$ & $x$ & $\mathrm{x}$ \\
\hline VIDEO CARD, NVIDIA QUADRO, 256MB & Hewlett Packard & GN493AV & EA & Site & $1-2$ & $\$ 49.00$ & $x$ & $x$ & $x$ \\
\hline VIDEO CARD, NVIDIA QUADRO, 256MB & Hewlett Packard & NVS295 & EA & Site & $1-2$ & $\$ 132.00$ & $x$ & $x$ & $\mathrm{x}$ \\
\hline VIDEO CARD, NVIDIA QUADRO, 256MB & Hewlett Packard & GN493AV & EA & Site & 1 & $\$ 49.00$ & $x$ & $x$ & $\mathrm{x}$ \\
\hline VIDEO DISTRIBUTER & HTS & 910371 & EA & $10 \mathrm{OCR}$ & 3 & $\$ 180.00$ & $x$ & $x$ & $\mathrm{x}$ \\
\hline VOLTAGE PROTECTOR, $230 \mathrm{~V}$ & Siemens & & EA & $10 \mathrm{OCR}$ & 4 & $\$ 136.13$ & $x$ & $x$ & $x$ \\
\hline VOLTAGE PROTECTOR, CAT6 & Siemens & & EA & 10 OCR & 4 & $\$ 66.72$ & $x$ & $x$ & $\mathrm{x}$ \\
\hline
\end{tabular}




\begin{tabular}{|c|c|c|c|c|c|c|c|c|c|}
\hline Nomenclature & Manufacturer & Part Number & UI & Basis per & $B Q$ & Cost & $\mathrm{SH}$ & $\mathrm{R}$ & $\mathrm{O}$ \\
\hline VOLTAGE REGULATOR & Corcom & 3VDK1 [or 6VN1] & EA & 10 Systems & 1 & $\$ 40.00$ & $x$ & $x$ & $\mathrm{O}$ \\
\hline $\begin{array}{l}\text { VOLTAGE REGULATOR, OUTDOOR, } 172 \text { TO } \\
276 \text { VAC INPUT }\end{array}$ & TSi Power & VRx-2100-8071 & EA & 10 Systems & 1 & $\$ 200.00$ & $x$ & $x$ & $x$ \\
\hline VPN APPLIANCE & Cisco & & EA & Site & 1 & $\$ 1,400.00$ & M & $x$ & $x$ \\
\hline WIRE, INDUCTION LOOP & Magnetic Autocontrol & 90000.0001 & EA & 10 Traffic Arms & 4 & $\$ 150.00$ & $x$ & $x$ & $x$ \\
\hline WIRELESS ACCESS POINT & D-Link & 0465 & EA & 10 CAS & 1 & $\$ 200.00$ & $x$ & $x$ & $x$ \\
\hline WORK STATION & Aspect & 425681.033-02 & KIT & & & & $x$ & $x$ & $x$ \\
\hline WORK STATION & Aspect & 425681.032 & KIT & & & & $x$ & $x$ & $\mathrm{x}$ \\
\hline WORK STATION & Hewlett Packard & DU937AV & KIT & Site & 1 & $\$ 399.55$ & $x$ & $x$ & $x$ \\
\hline WORK STATION & Hewlett Packard & ER236AV & KIT & Site & 1 & $\$ 244.77$ & $x$ & $x$ & $\mathrm{x}$ \\
\hline WORK STATION & Hewlett Packard & DU937AV & KIT & Site & $1-2$ & $\$ 399.55$ & $x$ & $x$ & $x$ \\
\hline WORK STATION & Hewlett Packard & RV725AV & KIT & Site & $1-2$ & $\$ 644.99$ & $x$ & $x$ & $x$ \\
\hline WORK STATION & Hewlett Packard & RV725AV & KIT & Site & 1 & $\$ 644.99$ & $x$ & $x$ & $x$ \\
\hline WORK STATION & Hewlett Packard & RV725AV & KIT & Site & 1 & $\$ 644.99$ & $x$ & $x$ & $\mathrm{x}$ \\
\hline WORK STATION & Hewlett Packard & & KIT & Site & $1-2$ & $\$ 2,063.00$ & M & $x$ & $x$ \\
\hline WORK STATION & Hewlett Packard & & KIT & Site & 1 & $\$ 1,159.00$ & M & $x$ & $x$ \\
\hline WORK STATION & Hewlett Packard & & KIT & Site & 1 & $\$ 2,063.00$ & $M$ & $x$ & $x$ \\
\hline WORK STATION & Hewlett Packard & $\begin{array}{l}\text { HP 5100MT + } \\
\text { Windows XP }\end{array}$ & EA & & & $\$ 1,697.00$ & $x$ & $x$ & $x$ \\
\hline $\begin{array}{l}\text { WORK STATION, OCR, ACQUISITION AND } \\
\text { WORKER }\end{array}$ & Generic Manufacturer & $430676-002$ & KIT & $10 \mathrm{OCR}$ & 1 & $\$ 3,000.00$ & M & $x$ & $x$ \\
\hline WORK STATION, RAVEN & PC CITY BUILD & 0458 & KIT & 10 CAS & 1 & $\$ 1,722.50$ & M & $x$ & $\mathrm{O}$ \\
\hline $\begin{array}{l}\text { WORK STATION, WITH COVER, WITH } \\
\text { MOUSE }\end{array}$ & Cybernet & 0458A & KIT & 10 CAS & 1 & $\$ 1,742.50$ & M & $x$ & 0 \\
\hline
\end{tabular}




\section{Master Parts Catalog by Manufacturer}

\begin{tabular}{|c|c|c|c|c|c|}
\hline Nomenclature & Part Number & End Item & $\mathrm{SH}$ & $\mathrm{R}$ & $\mathrm{O}$ \\
\hline \multicolumn{6}{|l|}{ *** Unknown } \\
\hline FIBER PONE & $60-000381-02$ & Serco Wireless Network & $x$ & $x$ & $x$ \\
\hline MEDIA CONVERTER & $59190634-7$ & Schneider CAS & $x$ & $x$ & $x$ \\
\hline \multicolumn{6}{|l|}{$A B B$} \\
\hline CIRCUIT BREAKER, 2P, 40A & F2020AC-40/0.03 & Utility Panels & $x$ & $x$ & $x$ \\
\hline \multicolumn{6}{|l|}{ ADC } \\
\hline ACCESSORY, CABLE & ADCCMVIB-3CB20-4 & Siemens Wireless Network & $x$ & $x$ & $x$ \\
\hline \multicolumn{6}{|l|}{ Advantech } \\
\hline CONVERTER, IP & ADAM-6060-BE & Siemens Wireless Network & $x$ & $x$ & $x$ \\
\hline POWER SUPPLY, CONTROLLER & PWR-242 & Siemens Wireless Network & M & $x$ & $x$ \\
\hline SWITCH, ETHERNET, HARDENED & EKI-7758F & Serco Wireless Network & M & $x$ & $x$ \\
\hline \multicolumn{6}{|l|}{ AEG } \\
\hline CARD, UPS SNMP & & Siemens OCR & $x$ & $x$ & $x$ \\
\hline UPS, 3000VA, RACK & & Siemens OCR & $\mathrm{MH}$ & $x$ & $x$ \\
\hline \multicolumn{6}{|l|}{ AGSI } \\
\hline CABLE, DUPLEX, LC SC $2 \mathrm{M}$ & $810-147-006$ & Siemens Wireless Network & $x$ & $x$ & $x$ \\
\hline \multicolumn{6}{|l|}{ Allen Bradley } \\
\hline CIRCUIT BREAKER, 1P 230V AC, 20 & S201U-K20 & Utility Panels & $x$ & $x$ & $x$ \\
\hline CIRCUIT BREAKER, 1P 230V AC, 40A & S201U-K40 & Utility Panels & $x$ & $x$ & $x$ \\
\hline CIRCUIT BREAKER, 1P 230V AC, 50A & S201U-K50 & Utility Panels & $x$ & $x$ & $x$ \\
\hline CIRCUIT BREAKER, 1P 230V AC, 5A & S201U-K50 & Utility Panels & $x$ & $x$ & $x$ \\
\hline CIRCUIT BREAKER, 1P DIN RAIL MOUNT, 6A & 1492-SP1C060 & Utility Panels & $x$ & $x$ & $x$ \\
\hline CIRCUIT BREAKER, 1P PLUS NEUTRAL, 20A & 1492-SP1B200-N & Utility Panels & $x$ & $x$ & $x$ \\
\hline CIRCUIT BREAKER, 1P, 10A & 1492-SP1C100 & Utility Panels & $x$ & $x$ & $x$ \\
\hline CIRCUIT BREAKER, 2P, 50A & 1492-SP2C500 & Utility Panels & $x$ & $x$ & $x$ \\
\hline CIRCUIT BREAKER, 5A & 1492-SP1C050 & Utility Panels & $x$ & $x$ & $x$ \\
\hline
\end{tabular}




\begin{tabular}{|c|c|c|c|c|c|}
\hline Nomenclature & Part Number & End Item & $\mathrm{SH}$ & $\mathrm{R}$ & $\mathrm{O}$ \\
\hline MODULE, TIMING & 700-HT3 & Utility Panels & $x$ & $x$ & $x$ \\
\hline RELAY, TUBE BASE, WITH PILOT LIGHT & 700-HA33Z12-4 & Utility Panels & $x$ & $x$ & $x$ \\
\hline \multicolumn{6}{|l|}{ APC } \\
\hline BATTERY PACK & JB0829023294 & Schneider CAS & $\mathrm{H}$ & $x$ & $x$ \\
\hline BATTERY PACK, 192V, RM & SURT192MXLBP & Siemens Wireless Network & $\mathrm{H}$ & $x$ & $x$ \\
\hline BATTERY PACK, 24V, RS XS & BR24BP & Siemens Wireless Network & $\mathrm{H}$ & $x$ & $x$ \\
\hline CABLE, POWER, 16A 230V & AP9876 & Siemens Wireless Network & $x$ & $x$ & $x$ \\
\hline CABLE, POWER, AC & AP9870 & Siemens Wireless Network & $x$ & $x$ & $x$ \\
\hline NETWORK CARD, MANAGEMENT & & Schneider CAS & $x$ & $\mathrm{x}$ & $x$ \\
\hline UPS, 1400VA & SU1400RMXLB3U & Serco Wireless Network & $\mathrm{MH}$ & $x$ & 0 \\
\hline UPS, 1400VA & SUI400RMXLIB3U & Siemens Wireless Network & $\mathrm{MH}$ & $x$ & $x$ \\
\hline UPS, 1500 VA $120 \mathrm{~V}$ & & Schneider CAS & $\mathrm{H}$ & $x$ & $x$ \\
\hline UPS, 1500VA & $\begin{array}{l}1500 \text { (exact model } \\
\text { country/region } \\
\text { specific) }\end{array}$ & Serco CAS - Current & $\mathrm{H}$ & $x$ & $x$ \\
\hline UPS, 1500VA & $\begin{array}{l}1500 \text { (exact model } \\
\text { country/region } \\
\text { specific) }\end{array}$ & Serco CAS - Current & H & $x$ & $x$ \\
\hline UPS, 1500VA & $\begin{array}{l}1500 \text { (exact model } \\
\text { country/region } \\
\text { specific) }\end{array}$ & Serco CAS - Current & H & $x$ & $x$ \\
\hline UPS, 1500VA & BR15001 & Siemens Wireless Network & $\mathrm{H}$ & $x$ & $x$ \\
\hline UPS, 1500VA & varies & Serco CAS - 1 Generation Old & $\mathrm{H}$ & $x$ & $x$ \\
\hline UPS, 1500VA & varies & Serco CAS - 2 Generations Old & $\mathrm{H}$ & $x$ & $x$ \\
\hline UPS, 1500VA & varies & Serco CAS - 3 Generations Old & $\mathrm{H}$ & $x$ & $x$ \\
\hline UPS, 1500VA & varies & Serco CAS - 3 Generations Old & $\mathrm{H}$ & $\mathrm{x}$ & $x$ \\
\hline UPS, 1500VA & varies & Serco CAS - 2 Generations Old & H & $x$ & $x$ \\
\hline UPS, 1500VA & varies & Serco CAS - 1 Generation Old & $\mathrm{H}$ & $x$ & $x$ \\
\hline UPS, 1500VA & varies & Serco CAS - 1 Generation Old & $\mathrm{H}$ & $x$ & $x$ \\
\hline UPS, 3000VA & $\begin{array}{l}3000 \text { (exact model } \\
\text { country/region } \\
\text { specific) }\end{array}$ & Serco CAS - Current & H & $x$ & $x$ \\
\hline
\end{tabular}




\begin{tabular}{|c|c|c|c|c|c|}
\hline Nomenclature & Part Number & End Item & $\mathrm{SH}$ & $\mathrm{R}$ & $\mathrm{O}$ \\
\hline UPS, 3000VA & $\begin{array}{l}3000 \text { (exact model } \\
\text { country/region } \\
\text { specific) }\end{array}$ & Serco CAS - Current & $\mathrm{H}$ & $x$ & $x$ \\
\hline UPS, 3000VA & varies & Serco CAS - 3 Generations Old & $\mathrm{H}$ & $x$ & $x$ \\
\hline UPS, 3000VA & varies & Serco CAS - 2 Generations Old & $\mathrm{H}$ & $x$ & $x$ \\
\hline UPS, 3000VA & varies & Serco CAS - 1 Generation Old & $\mathrm{H}$ & $x$ & $x$ \\
\hline UPS, 3000VA & varies & Serco CAS - 2 Generations Old & $\mathrm{H}$ & $x$ & $x$ \\
\hline UPS, 3000VA & varies & Serco CAS - 1 Generation Old & $\mathrm{H}$ & $x$ & $x$ \\
\hline UPS, 3000VA & varies & Serco CAS - 3 Generations Old & $\mathrm{H}$ & $x$ & $x$ \\
\hline UPS, 5000VA & $\begin{array}{l}5000 \text { (exact model } \\
\text { country/region } \\
\text { specific) }\end{array}$ & Serco CAS - Current & $\mathrm{MH}$ & $x$ & $x$ \\
\hline UPS, 5000VA & varies & Serco CAS - 1 Generation Old & $\mathrm{MH}$ & $x$ & $x$ \\
\hline UPS, 5000VA & varies & Serco CAS - 2 Generations Old & $\mathrm{MH}$ & $x$ & $x$ \\
\hline UPS, 5000VA & varies & Serco CAS - 3 Generations Old & $\mathrm{MH}$ & $x$ & $x$ \\
\hline UPS, 6000VA & SURT6000XLI & Siemens Wireless Network & $\mathrm{MH}$ & $\mathrm{x}$ & $\mathrm{x}$ \\
\hline \multicolumn{6}{|l|}{ APS Technology } \\
\hline BOARD, SINGLE, IO TO ETHERNET & 24-016-015 & APS OCR & $x$ & $x$ & $x$ \\
\hline CAMERA, TOP LENS MOUNTED, 3.0MP & 29-0019-001 & APS OCR & M & $\mathrm{x}$ & $x$ \\
\hline CAMERA, TOP LENS MOUNTED, 3.0MP & 29002-001 & APS OCR & M & $x$ & $x$ \\
\hline DETECTOR, LOOP & 24-0016-014 & APS OCR & $x$ & $x$ & $x$ \\
\hline DETECTOR, LOOP, EXTENDED PRESENCE & $42-0002-001$ & APS OCR & $x$ & $\mathrm{x}$ & $\mathrm{x}$ \\
\hline DETECTOR, RADAR, FRONT AND REAR SPEED & 80-0001-001 & APS OCR & $x$ & $x$ & $x$ \\
\hline ILLUMINATOR, SIDE FLAT ARRAY & $66-0003001$ & APS OCR & $x$ & $\mathrm{x}$ & $\mathrm{X}$ \\
\hline ILLUMINATOR, SIDE FLAT ARRAY & $66-0003002$ & APS OCR & $x$ & $x$ & $\mathrm{x}$ \\
\hline MEDIA CONVERTER, TRANSCEIVER MODULE, MULTIMODE & & APS OCR & M & $x$ & $\mathrm{X}$ \\
\hline POWER SUPPLY, 12 VDC & 24-0016-029 & APS OCR & $x$ & $x$ & $x$ \\
\hline POWER SUPPLY, 24 VDC & 24-0016-030 & APS OCR & $\mathrm{x}$ & $\mathrm{x}$ & $\mathrm{x}$ \\
\hline RECEPTACLE, REMOTELY MANAGED & 24-0016-018 & APS OCR & $x$ & $\mathrm{x}$ & $\mathrm{x}$ \\
\hline SENSOR, BEAM RECEIVER AND TRANSMITTER & 44-0001-001 & APS OCR & $x$ & $x$ & $x$ \\
\hline
\end{tabular}




\begin{tabular}{|c|c|c|c|c|c|}
\hline Nomenclature & Part Number & End Item & $\mathrm{SH}$ & $\mathrm{R}$ & 0 \\
\hline SERVER, OCS OCR & 00-0001-003 & APS OCR & M & $x$ & $x$ \\
\hline SWITCH, 24 PORT, GIGABIT & 56-0015-002 & APS OCR & $x$ & $x$ & $x$ \\
\hline \multicolumn{6}{|l|}{ ARC Wireless } \\
\hline MOUNT, RADIO, OUTDOOR & 0466M & RAVEN CAS & $\mathrm{x}$ & $\mathrm{x}$ & $x$ \\
\hline \multicolumn{6}{|l|}{ Automation Direct } \\
\hline CABLE, PC/PLC PROGRAM, 9PIN SERIAL TO RJ45 & D2-DSCBL & Utility Panels & $x$ & $x$ & $x$ \\
\hline CONTROLLER, PROGRAMMABLE LOGIC & D0-060DR-D & Utility Panels & $x$ & $\mathrm{x}$ & $x$ \\
\hline KIT, CABLE, PLC PROGRAMMING & DA-DSCBL & Utility Panels & $x$ & $x$ & $\mathrm{x}$ \\
\hline MODULE, COMMUNICATION, ETHERNET & HO-ECOM100 & Utility Panels & $x$ & $\mathrm{x}$ & $x$ \\
\hline PROGRAMMABLE LOGIC CONTROLLER HAND HELD & D2-HPP & Utility Panels & $\mathrm{x}$ & $x$ & $\mathrm{x}$ \\
\hline SOFTWARE, PLC PROGRAMMING & PC-DSOFT5 & Utility Panels & $x$ & $x$ & $x$ \\
\hline \multicolumn{6}{|l|}{ Belkin } \\
\hline CABLE, CAT5E & UTP 4 pairs Cat5e & Utility Panels & $x$ & $\mathrm{x}$ & $x$ \\
\hline CABLE, CAT5E, RJ-45 & & Schneider CAS & $x$ & $x$ & $x$ \\
\hline \multicolumn{6}{|l|}{ B-Line } \\
\hline HEATER, CONVECTION, 30W & $\mathrm{EH}-30$ & Utility Panels & $x$ & $x$ & $\mathrm{x}$ \\
\hline HEATER, CONVECTION, 6OW & CAW EH-60 & Utility Panels & $x$ & $x$ & $x$ \\
\hline \multicolumn{6}{|l|}{ Bosch } \\
\hline ILLUMINATOR, IR & & RAVEN CAS & $x$ & $\mathrm{x}$ & $x$ \\
\hline ILLUMINATOR, IR, 850NM & EX26-LED-850M & Siemens Wireless Network & M & $x$ & $x$ \\
\hline \multicolumn{6}{|l|}{ Bussman } \\
\hline FUSE, $12 \mathrm{~V}$ DC $5 \mathrm{~A}$ & AGC-5 & Utility Panels & $x$ & $x$ & $x$ \\
\hline FUSE, 15A & LP-CC-15 & Utility Panels & $x$ & $x$ & $x$ \\
\hline \multicolumn{6}{|l|}{ Cables Plus } \\
\hline PANEL INSERT, PATCH, FIBER & AP-VCC1CB & Utility Panels & $x$ & $x$ & $x$ \\
\hline \multicolumn{6}{|l|}{ Cisco } \\
\hline FIREWALL & ASA5505-50-BUN-K8 & Siemens Wireless Network & $x$ & $\mathrm{x}$ & $\mathrm{x}$ \\
\hline
\end{tabular}




\begin{tabular}{|c|c|c|c|c|c|}
\hline Nomenclature & Part Number & End Item & $\mathrm{SH}$ & $\mathrm{R}$ & $\mathrm{O}$ \\
\hline FIREWALL & varies & Serco CAS - 2 Generations Old & M & $x$ & $x$ \\
\hline FIREWALL & varies & Serco CAS - 1 Generation Old & M & $x$ & $x$ \\
\hline FIREWALL, ASA 5505 & ASA 5505 & Serco CAS - Current & $x$ & $x$ & $x$ \\
\hline KIT, VOIP PHONE AND LICENSE & CP-7941G-A-CCME & Siemens Wireless Network & $x$ & $x$ & $x$ \\
\hline LIGHTNING ARRESTER & 60-000381-01 & Serco Wireless Network & $x$ & $x$ & $x$ \\
\hline ROUTER & varies & Serco CAS - 1 Generation Old & $\mathrm{x}$ & $\mathrm{x}$ & $\mathrm{x}$ \\
\hline ROUTER & varies & Serco CAS - 3 Generations Old & $x$ & $x$ & $x$ \\
\hline ROUTER & varies & Serco CAS - 2 Generations Old & $x$ & $x$ & $x$ \\
\hline ROUTER, 2821 & 2821 & Serco CAS - Current & M & $x$ & $x$ \\
\hline SERVER & & SafePass CAS & $x$ & $x$ & $x$ \\
\hline SERVER & & SafePass CAS & $x$ & $x$ & $x$ \\
\hline SOFTWARE, SECURITY & 2821-HSEC/K9 & Siemens Wireless Network & M & $x$ & $x$ \\
\hline SWITCH, 1000 BASE T & GLC-T & Siemens Wireless Network & $x$ & $x$ & $x$ \\
\hline SWITCH, 24 PORT & $2960-24$ & Serco CAS - Current & $x$ & $x$ & $x$ \\
\hline SWITCH, 24 PORT & $2960-24$ & Serco CAS - Current & M & $x$ & $x$ \\
\hline SWITCH, 24 PORT & $2960-24$ & Serco CAS - Current & $x$ & $x$ & $x$ \\
\hline SWITCH, 24 PORT & varies & Serco CAS - 2 Generations Old & M & $x$ & $x$ \\
\hline SWITCH, 24 PORT & varies & Serco CAS - 1 Generation Old & $x$ & $x$ & $x$ \\
\hline SWITCH, 24 PORT & varies & Serco CAS - 1 Generation Old & $x$ & $x$ & $x$ \\
\hline SWITCH, 24 PORT & varies & Serco CAS - 3 Generations Old & M & $x$ & $x$ \\
\hline SWITCH, 24 PORT & varies & Serco CAS - 1 Generation Old & M & $x$ & $x$ \\
\hline SWITCH, 24 PORT & varies & Serco CAS - 2 Generations Old & M & $x$ & $x$ \\
\hline SWITCH, 24 PORT & varies & Serco CAS - 3 Generations Old & M & $x$ & $x$ \\
\hline SWITCH, 24 PORT & varies & Serco CAS - 1 Generation Old & $x$ & $x$ & $x$ \\
\hline SWITCH, 24 PORT & varies & Serco CAS - 1 Generation Old & $x$ & $x$ & $\mathrm{x}$ \\
\hline SWITCH, 48 PORT & $3750-48$ & Serco CAS - Current & M & $\mathrm{x}$ & $\mathrm{x}$ \\
\hline SWITCH, 48 PORT & $3750-48$ & Serco CAS - Current & M & $x$ & $x$ \\
\hline SWITCH, 48 PORT & varies & Serco CAS - 2 Generations Old & M & $x$ & $x$ \\
\hline SWITCH, 48 PORT & varies & Serco CAS - 3 Generations Old & M & $\mathrm{x}$ & $x$ \\
\hline
\end{tabular}




\begin{tabular}{|c|c|c|c|c|c|}
\hline Nomenclature & Part Number & End Item & $\mathrm{SH}$ & $\mathrm{R}$ & $\mathrm{O}$ \\
\hline SWITCH, 48 PORT & varies & Serco CAS - 3 Generations Old & M & $\mathrm{x}$ & $\mathrm{x}$ \\
\hline SWITCH, 48 PORT & varies & Serco CAS - 1 Generation Old & M & $\mathrm{x}$ & $\mathrm{x}$ \\
\hline SWITCH, 48 PORT & varies & Serco CAS - 1 Generation Old & $x$ & $x$ & $x$ \\
\hline SWITCH, 48 PORT & varies & Serco CAS - 1 Generation Old & M & $x$ & $x$ \\
\hline SWITCH, 48 PORT & varies & Serco CAS - 2 Generations Old & M & $\mathrm{x}$ & $\mathrm{x}$ \\
\hline SWITCH, CATALYST & WS-C2955T-12 & Serco Wireless Network & M & $x$ & $x$ \\
\hline SWITCH, CATALYST & WS-C3750-24FS-E & Siemens Wireless Network & M & $x$ & $\mathrm{x}$ \\
\hline SWITCH, CATALYST & WS-C3750G-12S-E & Serco Wireless Network & $x$ & $x$ & $x$ \\
\hline SWITCH, CATALYST & WS-C3750G-24PS-E & Siemens Wireless Network & M & $x$ & $x$ \\
\hline SWITCH, CATALYST & WS-C3750G-24T-E & Serco Wireless Network & M & $x$ & $x$ \\
\hline SWITCH, RACK MOUNT, 24 PORT & & SafePass CAS & M & $x$ & $x$ \\
\hline SWITCH, RACK MOUNT, 48 PORT & & SafePass CAS & M & $\mathrm{x}$ & $\mathrm{x}$ \\
\hline TELEPHONY, VOIP & SPA901 & Schneider CAS & $x$ & $x$ & $x$ \\
\hline TRANCEIVER, FIBER CONVERTER, LXLH & GLC-LH-SM & ICIS OCR & $x$ & $x$ & $x$ \\
\hline TRANCEIVER, LC CONNECTOR SX & GLC-SX-MM & Siemens Wireless Network & $\mathrm{x}$ & $\mathrm{x}$ & $x$ \\
\hline VPN APPLIANCE & & SafePass CAS & M & $x$ & $x$ \\
\hline \multicolumn{6}{|l|}{ Corcom } \\
\hline VOLTAGE REGULATOR & 3VDK1 [or 6VN1] & Utility Panels & $x$ & $x$ & 0 \\
\hline \multicolumn{6}{|l|}{ CTL } \\
\hline MONITOR, FLAT PANEL, 19 INCH & 0455 & RAVEN CAS & $x$ & $x$ & $x$ \\
\hline \multicolumn{6}{|l|}{ Cybernet } \\
\hline WORK STATION, WITH COVER, WITH MOUSE & 0458A & RAVEN CAS & M & $x$ & 0 \\
\hline \multicolumn{6}{|l|}{ Deliberant } \\
\hline RADIO, OUTDOOR & 0465B & RAVEN CAS & $\mathrm{x}$ & $x$ & $\mathrm{x}$ \\
\hline RADIO, OUTDOOR & 0466 & RAVEN CAS & $x$ & $x$ & $x$ \\
\hline SURGE PROTECTOR & 0466A & RAVEN CAS & $x$ & $x$ & $x$ \\
\hline \multicolumn{6}{|l|}{ Dell } \\
\hline CABLE, PRINTER, USB 10 FT & $310-8688$ & Schneider CAS & $\mathrm{x}$ & $\mathrm{x}$ & $\mathrm{x}$ \\
\hline
\end{tabular}




\begin{tabular}{|c|c|c|c|c|c|}
\hline Nomenclature & Part Number & End Item & $\mathrm{SH}$ & $\mathrm{R}$ & 0 \\
\hline KEYBOARD, USB & $310-7949$ & Schneider CAS & $\mathrm{x}$ & $\mathrm{x}$ & $\mathrm{x}$ \\
\hline MONITOR, FLAT PANEL & 1908FP-BLK & Schneider CAS & $x$ & $x$ & $x$ \\
\hline MONITOR, FLAT PANEL & 2209WA & Schneider CAS & $\mathrm{x}$ & $\mathrm{x}$ & $x$ \\
\hline MOUSE, USB, OPTICAL & $310-9602$ & Schneider CAS & $\mathrm{x}$ & $\mathrm{x}$ & $x$ \\
\hline PRINTER & GQ4BPG1 & Schneider CAS & $x$ & $x$ & $x$ \\
\hline PRINTER, MULTIFUNCTION, COLOR LASER & $3115 \mathrm{CN}$ & Schneider CAS & $x$ & $x$ & $x$ \\
\hline SPEAKER, SOUND BAR & $313-6414$ & Schneider CAS & $x$ & $x$ & $x$ \\
\hline SWITCH, 24 PORT & & Schneider CAS & $\mathrm{x}$ & $\mathrm{x}$ & $\mathrm{x}$ \\
\hline SWITCH, 8 PORT, GBE WEB MANAGED & FPX9FH1 & Schneider CAS & $x$ & $x$ & $\mathrm{x}$ \\
\hline WORK STATION, MINITOWER & 1J3SNJ1 & Schneider CAS & $\mathrm{x}$ & $\mathrm{x}$ & $\mathrm{x}$ \\
\hline \multicolumn{6}{|l|}{ D-Link } \\
\hline ANTENNA, EXTERNAL & 0465A & RAVEN CAS & $\mathrm{x}$ & $x$ & $x$ \\
\hline WIRELESS ACCESS POINT & 0465 & RAVEN CAS & $x$ & $x$ & $x$ \\
\hline \multicolumn{6}{|l|}{ Dragonwave } \\
\hline ANTENNA, 26GHZ, 0.3M & VHLP1-26-DW1 & Serco Wireless Network & $\mathrm{x}$ & $\mathrm{x}$ & $\mathrm{x}$ \\
\hline ANTENNA, $28 \mathrm{GHZ}, 0.3 \mathrm{M}$ & VHLP1-28-DW1 & Serco Wireless Network & $x$ & $x$ & $x$ \\
\hline ANTENNA, 28GHZ, 0.6M & VHLP2-28-DW1 & Serco Wireless Network & $\mathrm{x}$ & $\mathrm{x}$ & $\mathrm{x}$ \\
\hline RADIO, COMPACT, 24GHZ & CUSP24UFC & Serco Wireless Network & M & $\mathrm{x}$ & $\mathrm{x}$ \\
\hline RADIO, COMPACT, 24GHZ & CUSP24UFF & Serco Wireless Network & M & $x$ & $\mathrm{x}$ \\
\hline RADIO, COMPACT, 28GHZ & CHHP28B1C & Serco Wireless Network & M & $x$ & $x$ \\
\hline RADIO, COMPACT, 28GHZ & CHHP28B2C & Serco Wireless Network & $M$ & $x$ & $x$ \\
\hline RADIO, COMPACT, 28GHZ & CLHP28B1C & Serco Wireless Network & M & $x$ & $x$ \\
\hline RADIO, COMPACT, 28GHZ & CLHP28B1F & Serco Wireless Network & $M$ & $x$ & $x$ \\
\hline RADIO, COMPACT, 28GHZ & CLHP28B2C & Serco Wireless Network & M & $\mathrm{x}$ & $\mathrm{x}$ \\
\hline RADIO, COMPACT, 28GHZ & CLHP28B2F & Serco Wireless Network & M & $x$ & $x$ \\
\hline TEST BENCH, 28GHZ & $60-000327-08-01$ & Serco Wireless Network & $\mathrm{x}$ & $x$ & $x$ \\
\hline \multicolumn{6}{|l|}{ ELO } \\
\hline MONITOR, TOUCH & ELO 1727 & Serco CAS - 2 Generations Old & $\mathrm{x}$ & $\mathrm{x}$ & $\mathrm{x}$ \\
\hline
\end{tabular}




\begin{tabular}{|c|c|c|c|c|c|}
\hline Nomenclature & Part Number & End Item & SH & $\mathrm{R}$ & $\mathrm{O}$ \\
\hline MONITOR, TOUCH & ELO 1727 & Serco CAS - 3 Generations Old & $x$ & $x$ & $\mathrm{x}$ \\
\hline \multicolumn{6}{|l|}{ Enlight } \\
\hline SERVER, RACK MOUNT, $2 U$ & 0459A & RAVEN CAS & M & $\mathrm{x}$ & $\mathrm{x}$ \\
\hline \multicolumn{6}{|l|}{ Exceltech } \\
\hline INVERTER, SINEWAVE & XP-125-12 & Schneider CAS & $x$ & $\mathrm{x}$ & $x$ \\
\hline \multicolumn{6}{|l|}{ Extreme CCTV } \\
\hline ILLUMINATOR, IR & 0460A & RAVEN CAS & $x$ & $x$ & $\mathrm{x}$ \\
\hline \multicolumn{6}{|l|}{ Finder } \\
\hline RELAY BASE & 90.03 & Utility Panels & $x$ & $x$ & $x$ \\
\hline \multicolumn{6}{|l|}{ FIS } \\
\hline CABLE, DUPLEX MALE TO MALE, 1M & R2MJYM1FISP & Siemens Wireless Network & $\mathrm{x}$ & $\mathrm{x}$ & $\mathrm{x}$ \\
\hline CABLE, DUPLEX, LC SC 2M & D3LYS2FISC & Siemens Wireless Network & $\mathrm{x}$ & $x$ & $\mathrm{x}$ \\
\hline \multicolumn{6}{|l|}{ GarrettCom } \\
\hline CONVERTER, MULTI MODE, WITH SC CONNECTOR & & SafePass CAS & $x$ & $x$ & $x$ \\
\hline CONVERTER, SINGLE MODE, WITH SC CONNECTOR & & SafePass CAS & $\mathrm{x}$ & $\mathrm{x}$ & $x$ \\
\hline MEDIA CONVERTER & CS14-1SC-i & Utility Panels & $x$ & $\mathrm{x}$ & $\mathrm{x}$ \\
\hline MOUNT, DIN RAIL & 0464BD & RAVEN CAS & $x$ & $x$ & $x$ \\
\hline POWER SUPPLY, SWITCH & $0464 \mathrm{C}$ & RAVEN CAS & $\mathrm{x}$ & $\mathrm{x}$ & $\mathrm{x}$ \\
\hline SWITCH, 24 PORT & 0464E & RAVEN CAS & M & $x$ & $x$ \\
\hline SWITCH, 6 PORT, 12 VDC & 0464B & RAVEN CAS & $x$ & $x$ & $x$ \\
\hline SWITCH, 8 PORT & 0464D & RAVEN CAS & $x$ & $x$ & $x$ \\
\hline SWITCH, CONVERTER, MULTIMODE & CS14H-SC-12VDC & Utility Panels & $x$ & $x$ & $x$ \\
\hline SWITCH, CONVERTER, SINGLE MODE & CS14H-SSC-12VDC & Utility Panels & $x$ & $x$ & $x$ \\
\hline SWITCH, ETHERNET & $\mathrm{ES} 42 \mathrm{H}-2 \mathrm{SSC}-\mathrm{Hi}$ & Utility Panels & $x$ & $x$ & $x$ \\
\hline SWITCH, MEDIA CONVERTER & CS14P-SC-12VDX & Utility Panels & $x$ & $\mathrm{x}$ & $x$ \\
\hline SWITCH, MEDIA CONVERTER & CSG14H-SX-HD & Serco Wireless Network & $x$ & $x$ & $x$ \\
\hline \multicolumn{6}{|l|}{ Generic Manufacturer } \\
\hline CABLE, MOLEX, CAT5E & PCD00174BK & Siemens Wireless Network & $\mathrm{x}$ & $\mathrm{x}$ & $\mathrm{x}$ \\
\hline
\end{tabular}




\begin{tabular}{|c|c|c|c|c|c|}
\hline Nomenclature & Part Number & End Item & $\mathrm{SH}$ & $\mathrm{R}$ & $\mathrm{O}$ \\
\hline CABLE, MOLEX, CAT5E & PCD00177BK & Siemens Wireless Network & $x$ & $x$ & $x$ \\
\hline CABLE, POWER, 3 FT & NA & Schneider CAS & $x$ & $x$ & $x$ \\
\hline COUPLER, BNC, FEMALE TO FEMALE & & ICIS OCR & $x$ & $\mathrm{x}$ & $x$ \\
\hline FUSE, 1.5A & GGC-1/2 & Utility Panels & $\mathrm{x}$ & $x$ & $x$ \\
\hline FUSE, 15A, CLASS CC & KTK-CC-15 & Utility Panels & $x$ & $x$ & $x$ \\
\hline FUSE, 5A & AGC-4 & Utility Panels & $x$ & $x$ & $x$ \\
\hline FUSE, 7A & GGC-7 & Utility Panels & $x$ & $x$ & $x$ \\
\hline HDD, DISK ARRAY, 450GB & & ICIS OCR & $\mathrm{x}$ & $\mathrm{x}$ & $\mathrm{x}$ \\
\hline HDD, REMOVABLE 15K, 146GB & $419814-001$ & ICIS OCR & $x$ & $x$ & $\mathrm{x}$ \\
\hline HUB, USB 2.0, 4 PORT & A1122455 & Schneider CAS & $\mathrm{x}$ & $\mathrm{x}$ & $\mathrm{x}$ \\
\hline SPLITTER, BNC, FEMAL TO MALE TO FEMALE & & ICIS OCR & $x$ & $x$ & $x$ \\
\hline WORK STATION, OCR, ACQUISITION AND WORKER & 430676-002 & ICIS OCR & M & $x$ & $x$ \\
\hline \multicolumn{6}{|l|}{ Genesis } \\
\hline HYGROSTAT, 120-230V, ETF012 & G01203.9-00 & Utility Panels & $\mathrm{x}$ & $\mathrm{x}$ & $\mathrm{x}$ \\
\hline \multicolumn{6}{|l|}{ George Fix Industries } \\
\hline SWITCH, TAMPER, MAGNETIC & 50F-12WG & Utility Panels & $x$ & $x$ & $x$ \\
\hline \multicolumn{6}{|l|}{ Grainger } \\
\hline PADLOCK, KEYED ALIKE & $4 \mathrm{YH} 24$ & Utility Panels & $\mathrm{x}$ & $\mathrm{x}$ & $\mathrm{x}$ \\
\hline \multicolumn{6}{|l|}{ Hewlett Packard } \\
\hline ADAPTER, INTERFACE, IP CONSOLE & & Serco CAS - Current & $x$ & $x$ & $x$ \\
\hline CHASSIS, 650W 80 PLUS POWER SUPPLY & GM505AV & Serco CAS - 1 Generation Old & $x$ & $x$ & $x$ \\
\hline CHASSIS, 650W 80 PLUS POWER SUPPLY & GM505AV & Serco CAS - 1 Generation Old & $x$ & $x$ & $x$ \\
\hline CHASSIS, 650W 80 PLUS POWER SUPPLY & GM505AV & Serco CAS - 1 Generation Old & $\mathrm{x}$ & $x$ & $\mathrm{x}$ \\
\hline CHASSIS, 650W 85\% EFFICIENCY POWER SUPPLY & & Serco CAS - Current & M & $x$ & $x$ \\
\hline CHASSIS, 650W 85\% EFFICIENCY POWER SUPPLY & & Serco CAS - Current & M & $\mathrm{x}$ & $\mathrm{x}$ \\
\hline CHASSIS, 650W 85\% EFFICIENCY POWER SUPPLY & & Serco CAS - Current & M & $\mathrm{x}$ & $\mathrm{x}$ \\
\hline CHASSIS, SERVER, PROLIANT & 371293-405 & Serco CAS - 2 Generations Old & $\mathrm{x}$ & $\mathrm{x}$ & $\mathrm{x}$ \\
\hline CHASSIS, SERVER, PROLIANT & 371293-405 & Serco CAS - 3 Generations Old & $\mathrm{x}$ & $\mathrm{x}$ & $\mathrm{x}$ \\
\hline
\end{tabular}




\begin{tabular}{|c|c|c|c|c|c|}
\hline Nomenclature & Part Number & End Item & $\mathrm{SH}$ & $\mathrm{R}$ & $\mathrm{O}$ \\
\hline CHASSIS, SERVER, PROLIANT & $371293-405$ & Serco CAS - 3 Generations Old & $x$ & $x$ & $x$ \\
\hline CHASSIS, SERVER, PROLIANT & $371293-405$ & Serco CAS - 2 Generations Old & $x$ & $x$ & $x$ \\
\hline CHASSIS, SERVER, PROLIANT & 391835-B21 & Serco CAS - 1 Generation Old & $x$ & $x$ & $x$ \\
\hline CHASSIS, SERVER, PROLIANT & 391835-B21 & Serco CAS - 1 Generation Old & $x$ & $x$ & $x$ \\
\hline CHASSIS, SERVER, PROLIANT & DL380G6 & Serco CAS - Current & M & $x$ & $x$ \\
\hline CHASSIS, SERVER, PROLIANT & DL380G6 & Serco CAS - Current & $\mathrm{M}$ & $x$ & $x$ \\
\hline $\mathrm{CPU}, \mathrm{X} 2.8$ & 397645-B21 & Serco CAS - 3 Generations Old & $x$ & $x$ & $x$ \\
\hline $\mathrm{CPU}, \mathrm{X} 2.8$ & 397645-B21 & Serco CAS - 2 Generations Old & $x$ & $x$ & $x$ \\
\hline $\mathrm{CPU}, \mathrm{X} 2.8$ & 397645-B21 & Serco CAS - 3 Generations Old & $x$ & $x$ & $x$ \\
\hline $\mathrm{CPU}, \mathrm{X} 2.8$ & 397645-B21 & Serco CAS - 2 Generations Old & $x$ & $x$ & $x$ \\
\hline $\mathrm{CPU}, \mathrm{X} 2.8$ & 397645-L22 & Serco CAS - 2 Generations Old & $x$ & $x$ & $x$ \\
\hline $\mathrm{CPU}, \mathrm{X} 2.8$ & 397645-L22 & Serco CAS - 3 Generations Old & $x$ & $x$ & $\mathrm{x}$ \\
\hline $\mathrm{CPU}, \mathrm{X} 2.8$ & 397645-L22 & Serco CAS - 3 Generations Old & $x$ & $x$ & $x$ \\
\hline $\mathrm{CPU}, \mathrm{X} 2.8$ & 397645-L22 & Serco CAS - 2 Generations Old & $x$ & $x$ & $x$ \\
\hline CPU, XEON 5110, CPU 1 & EX617AV & Serco CAS - 2 Generations Old & $x$ & $x$ & $x$ \\
\hline CPU, XEON 5110, CPU 1 & EX617AV & Serco CAS - 2 Generations Old & $x$ & $x$ & $x$ \\
\hline CPU, XEON 5110, CPU 2 & EX618AV & Serco CAS - 2 Generations Old & $x$ & $x$ & $x$ \\
\hline CPU, XEON 5110, CPU 2 & EX618AV & Serco CAS - 2 Generations Old & $x$ & $x$ & $x$ \\
\hline CPU, XEON E 5504, CPU 1 & E5504 & Serco CAS - Current & $x$ & $x$ & $x$ \\
\hline CPU, XEON E 5504, CPU 2 & E5504 & Serco CAS - Current & $\mathrm{x}$ & $x$ & $x$ \\
\hline CPU, XEON E5420, CPU 1 & 458577-L21 & Serco CAS - 1 Generation Old & $x$ & $x$ & $x$ \\
\hline CPU, XEON E5420, CPU 1 & 458577-L21 & Serco CAS - 1 Generation Old & $x$ & $x$ & $x$ \\
\hline CPU, XEON E5420, CPU 1 & GW497AV & Serco CAS - 1 Generation Old & $x$ & $x$ & $x$ \\
\hline CPU, XEON E5420, CPU 1 & GW497AV & Serco CAS - 1 Generation Old & $x$ & $x$ & $x$ \\
\hline CPU, XEON E5420, CPU 1 & GW497AV & Serco CAS - 1 Generation Old & $x$ & $x$ & $x$ \\
\hline CPU, XEON E5420, CPU 2 & 458577-B21 & Serco CAS - 1 Generation Old & M & $x$ & $x$ \\
\hline CPU, XEON E5420, CPU 2 & 458577-B21 & Serco CAS - 1 Generation Old & M & $x$ & $x$ \\
\hline CPU, XEON E5420, CPU 2 & GW498AV & Serco CAS - 1 Generation Old & $x$ & $x$ & $x$ \\
\hline CPU, XEON E5420, CPU 2 & GW498AV & Serco CAS - 1 Generation Old & $x$ & $x$ & $x$ \\
\hline
\end{tabular}




\begin{tabular}{|c|c|c|c|c|c|}
\hline Nomenclature & Part Number & End Item & $\mathrm{SH}$ & $\mathrm{R}$ & $\mathrm{O}$ \\
\hline CPU, XEON E5420, CPU 2 & GW498AV & Serco CAS - 1 Generation Old & $x$ & $x$ & $x$ \\
\hline CPU, XEON E5504, CPU 1 & E5504 & Serco CAS - Current & $x$ & $x$ & $x$ \\
\hline CPU, XEON E5504, CPU 1 & E5504 & Serco CAS - Current & $x$ & $x$ & $x$ \\
\hline CPU, XEON E5504, CPU 2 & E5504 & Serco CAS - Current & $x$ & $x$ & $x$ \\
\hline CPU, XEON E5504, CPU 2 & E5504 & Serco CAS - Current & $x$ & $x$ & $x$ \\
\hline CPU, XEON E5530, CPU 1 & DL380G6 & Serco CAS - Current & $x$ & $x$ & $x$ \\
\hline CPU, XEON E5530, CPU 1 & DL380G6 & Serco CAS - Current & $x$ & $x$ & $x$ \\
\hline CPU, XEON E5530, CPU 2 & DL380G6 & Serco CAS - Current & $x$ & $x$ & $x$ \\
\hline CPU, XEON E5530, CPU 2 & DL380G6 & Serco CAS - Current & $x$ & $x$ & $x$ \\
\hline CPU, XEON, 3.6GHZ & DY255AV & Serco CAS - 3 Generations Old & $x$ & $x$ & $x$ \\
\hline CPU, XEON, 3.6GHZ & DY255AV & Serco CAS - 3 Generations Old & $x$ & $x$ & $x$ \\
\hline ENCLOSURE, DRIVE & AD542B & Serco CAS - 1 Generation Old & $x$ & $x$ & $x$ \\
\hline ENCLOSURE, MSA, 60 SINGLE & 418408-B21 & Serco CAS - 3 Generations Old & M & $x$ & $x$ \\
\hline ENCLOSURE, MSA, 60 SINGLE & 418408-B21 & Serco CAS - 1 Generation Old & M & $x$ & $x$ \\
\hline ENCLOSURE, MSA, 60 SINGLE & 418408-B21 & Serco CAS - 2 Generations Old & M & $x$ & $x$ \\
\hline ENCLOSURE, MSA, 60 SINGLE & MSA-60 & Serco CAS - Current & M & $x$ & $x$ \\
\hline FLOPPY DRIVE & DY290AV & Serco CAS - 3 Generations Old & $x$ & $x$ & $x$ \\
\hline FLOPPY DRIVE & DY290AV & Serco CAS - 3 Generations Old & $x$ & $x$ & $x$ \\
\hline FLOPPY DRIVE & ER242AV & Serco CAS - 2 Generations Old & $x$ & $x$ & $x$ \\
\hline FLOPPY DRIVE & ER242AV & Serco CAS - 2 Generations Old & $x$ & $x$ & $x$ \\
\hline HDD ARRAY & AD554B & Serco CAS - 1 Generation Old & M & $x$ & $\mathrm{x}$ \\
\hline HDD CONTROLLER CARD & 291967-B21 & Serco CAS - 3 Generations Old & $x$ & $x$ & $x$ \\
\hline HDD CONTROLLER CARD & 291967-B21 & Serco CAS - 2 Generations Old & $x$ & $x$ & $x$ \\
\hline HDD CONTROLLER CARD & 291967-B21 & Serco CAS - 2 Generations Old & $x$ & $x$ & $x$ \\
\hline HDD CONTROLLER CARD & 291967-B21 & Serco CAS - 3 Generations Old & $x$ & $x$ & $x$ \\
\hline HDD CONTROLLER CARD & 337972-B21 & Serco CAS - 1 Generation Old & $x$ & $x$ & $x$ \\
\hline HDD CONTROLLER CARD & 381513-B21 & Serco CAS - 1 Generation Old & $x$ & $x$ & $x$ \\
\hline HDD CONTROLLER CARD & 411508-B21 & Serco CAS - 1 Generation Old & $x$ & $x$ & $x$ \\
\hline HDD CONTROLLER CARD & DZ540AV & Serco CAS - 3 Generations Old & $x$ & $x$ & $x$ \\
\hline
\end{tabular}




\begin{tabular}{|c|c|c|c|c|c|}
\hline Nomenclature & Part Number & End Item & $\mathrm{SH}$ & $\mathrm{R}$ & $\mathrm{O}$ \\
\hline HDD CONTROLLER CARD & DZ540AV & Serco CAS - 3 Generations Old & $x$ & $x$ & $x$ \\
\hline HDD CONTROLLER CARD, 4 PORT RAID, SAS SATA & & Serco CAS - Current & $x$ & $\mathrm{x}$ & $x$ \\
\hline HDD CONTROLLER CARD, 4 PORT RAID, SAS SATA & $3041 \mathrm{E}$ & Serco CAS - Current & $x$ & $\mathrm{x}$ & $x$ \\
\hline HDD CONTROLLER CARD, 4 PORT RAID, SAS SATA & $3041 \mathrm{E}$ & Serco CAS - Current & $x$ & $x$ & $\mathrm{X}$ \\
\hline HDD CONTROLLER CARD, BBWC, E200/128 & 411508-B21 & Serco CAS - 1 Generation Old & $x$ & $x$ & $\mathrm{x}$ \\
\hline HDD CONTROLLER CARD, P600/256 & 337972-B21 & Serco CAS - 1 Generation Old & $x$ & $x$ & $x$ \\
\hline HDD CONTROLLER CARD, P800/512 & 381513-B21 & Serco CAS - 1 Generation Old & $\mathrm{x}$ & $x$ & $\mathrm{X}$ \\
\hline HDD CONTROLLER CARD, SAS 2 PORT INT AND EXT, P800 512 BBWC & DL380G6 & Serco CAS - Current & $\mathrm{x}$ & $x$ & $\mathrm{x}$ \\
\hline HDD CONTROLLER CARD, SAS 2 PORT INT AND EXT, P800 512 BBWC & DL380G6 & Serco CAS - Current & $x$ & $x$ & $x$ \\
\hline HDD, FC 10K, 300GB & 364622-B23 & Serco CAS - 1 Generation Old & $\mathrm{x}$ & $\mathrm{x}$ & $x$ \\
\hline HDD, SAS 10K, 146GB & 286716-B22 & Serco CAS - 3 Generations Old & $x$ & $\mathrm{x}$ & $x$ \\
\hline HDD, SAS 10K, 146GB & 286716-B22 & Serco CAS - 3 Generations Old & $x$ & $\mathrm{x}$ & $x$ \\
\hline HDD, SAS 10K, 146GB & 286716-B22 & Serco CAS - 2 Generations Old & $x$ & $x$ & $x$ \\
\hline HDD, SAS 10K, 146GB & 286716-B22 & Serco CAS - 2 Generations Old & $x$ & $x$ & $x$ \\
\hline HDD, SAS 10K, 146GB & 507125-B21 & Serco CAS - 1 Generation Old & $x$ & $x$ & $x$ \\
\hline HDD, SAS 10K, 146GB & 507125-B21 & Serco CAS - 1 Generation Old & $x$ & $x$ & $x$ \\
\hline HDD, SAS 10K, 146GB & DL380G6 & Serco CAS - Current & $x$ & $x$ & $x$ \\
\hline HDD, SAS 10K, 146GB & DL380G6 & Serco CAS - Current & $x$ & $x$ & $x$ \\
\hline HDD, SAS 15K, 146GB & & Serco CAS - Current & $x$ & $x$ & $x$ \\
\hline HDD, SAS 15K, 146GB & & Serco CAS - Current & $x$ & $x$ & $x$ \\
\hline HDD, SAS 15K, 146GB & & Serco CAS - Current & $x$ & $x$ & $x$ \\
\hline HDD, SAS 15K, 146GB & & Serco CAS - Current & $x$ & $x$ & $x$ \\
\hline HDD, SAS 15K, 146GB & & Serco CAS - Current & $x$ & $x$ & $x$ \\
\hline HDD, SAS 15K, 146GB & & Serco CAS - Current & $x$ & $x$ & $x$ \\
\hline HDD, SAS 7.2K, $1 \mathrm{~TB}$ & 461137-B21 & Serco CAS - 1 Generation Old & $x$ & $x$ & $x$ \\
\hline HDD, SAS 7.2K, 1TB & 461137-B21 & Serco CAS - 2 Generations Old & $x$ & $x$ & $x$ \\
\hline HDD, SAS 7.2K, 1TB & 461137-B21 & Serco CAS - 3 Generations Old & $x$ & $x$ & $x$ \\
\hline HDD, SAS 7.2K, 1TB & MSA-60 & Serco CAS - Current & $x$ & $x$ & $x$ \\
\hline HDD, SATA 7200, 250GB & GM539AV & Serco CAS - 1 Generation Old & $x$ & $x$ & $x$ \\
\hline
\end{tabular}




\begin{tabular}{|c|c|c|c|c|c|}
\hline Nomenclature & Part Number & End Item & $\mathrm{SH}$ & $\mathrm{R}$ & $\mathrm{O}$ \\
\hline HDD, SATA 7200, 250GB & GM539AV & Serco CAS - 1 Generation Old & $x$ & $x$ & $x$ \\
\hline HDD, SATA 7200, 250GB & GM539AV & Serco CAS - 1 Generation Old & $x$ & $x$ & $x$ \\
\hline HDD, SATA 7200, 250GB & GM539AV & Serco CAS - 1 Generation Old & $x$ & $x$ & $x$ \\
\hline HDD, SATA 7200, 250GB & GM539AV & Serco CAS - 1 Generation Old & $x$ & $x$ & $x$ \\
\hline HDD, SATA 7200, 250GB & GM539AV & Serco CAS - 1 Generation Old & $x$ & $x$ & $x$ \\
\hline HDD, SATA $7200,80 G B$ & ER249AV & Serco CAS - 2 Generations Old & $x$ & $x$ & $x$ \\
\hline HDD, SATA 7200, 80GB & ER249AV & Serco CAS - 2 Generations Old & $x$ & $x$ & $x$ \\
\hline HDD, SATA $7200,80 G B$ & ER259AV & Serco CAS - 2 Generations Old & $x$ & $x$ & $x$ \\
\hline HDD, SATA 7200, 80GB & ER259AV & Serco CAS - 2 Generations Old & $x$ & $x$ & $x$ \\
\hline HDD, SCSI 15K, 36GB & DY277AV & Serco CAS - 3 Generations Old & $x$ & $x$ & $x$ \\
\hline HDD, SCSI 15K, 36GB & DY277AV & Serco CAS - 3 Generations Old & $x$ & $x$ & $x$ \\
\hline HDD, SCSI 15K, 36GB & DY277AV & Serco CAS - 3 Generations Old & $x$ & $x$ & $x$ \\
\hline HDD, SCSI 15K, 36GB & DY277AV & Serco CAS - 3 Generations Old & $x$ & $x$ & $x$ \\
\hline INTERFACE ADAPTER & 262588-B21 & Serco CAS - 1 Generation Old & $x$ & $x$ & $x$ \\
\hline KEYBOARD, PS2 & & Serco CAS - Current & $x$ & $x$ & $x$ \\
\hline KEYBOARD, PS2 & & Serco CAS - Current & $x$ & $x$ & $x$ \\
\hline KEYBOARD, PS2 & & Serco CAS - Current & $x$ & $x$ & $x$ \\
\hline KEYBOARD, PS2 & DZ204AV\#ABA & Serco CAS - 2 Generations Old & $x$ & $x$ & $x$ \\
\hline KEYBOARD, PS2 & DZ204AV\#ABA & Serco CAS - 3 Generations Old & $x$ & $x$ & $x$ \\
\hline KEYBOARD, PS2 & DZ204AV\#ABA & Serco CAS - 2 Generations Old & $x$ & $x$ & $x$ \\
\hline KEYBOARD, PS2 & DZ204AV\#ABA & Serco CAS - 3 Generations Old & $x$ & $x$ & $x$ \\
\hline KEYBOARD, PS2 & GM604AV\#ABA & Serco CAS - 1 Generation Old & $x$ & $x$ & $x$ \\
\hline KEYBOARD, PS2 & GM604AV\#ABA & Serco CAS - 1 Generation Old & $x$ & $x$ & $x$ \\
\hline KEYBOARD, PS2 & GM604AV\#ABA & Serco CAS - 1 Generation Old & $x$ & $x$ & $x$ \\
\hline KIT, 6 FAN RACK TRAY, 220V & 257414-B21 & Serco Wireless Network & $x$ & $x$ & $x$ \\
\hline KIT, LOCALIZATION & & Serco CAS - Current & $x$ & $x$ & $x$ \\
\hline KIT, LOCALIZATION & & Serco CAS - Current & $x$ & $x$ & $x$ \\
\hline KIT, LOCALIZATION & & Serco CAS - Current & $x$ & $x$ & $x$ \\
\hline KIT, LOCALIZATION & DY250AV\#ABA & Serco CAS - 3 Generations Old & $x$ & $x$ & $x$ \\
\hline
\end{tabular}




\begin{tabular}{|c|c|c|c|c|c|}
\hline Nomenclature & Part Number & End Item & $\mathrm{SH}$ & $\mathrm{R}$ & $\mathrm{O}$ \\
\hline KIT, LOCALIZATION & DY250AV\#ABA & Serco CAS - 3 Generations Old & $x$ & $x$ & $x$ \\
\hline KIT, LOCALIZATION & ER296AV\#ABA & Serco CAS - 2 Generations Old & $x$ & $x$ & $x$ \\
\hline KIT, LOCALIZATION & ER296AV\#ABA & Serco CAS - 2 Generations Old & $x$ & $x$ & $x$ \\
\hline KIT, LOCALIZATION & GM506AV\#ABA & Serco CAS - 1 Generation Old & $x$ & $x$ & $x$ \\
\hline KIT, LOCALIZATION & GM506AV\#ABA & Serco CAS - 1 Generation Old & $x$ & $x$ & $x$ \\
\hline KIT, LOCALIZATION & GM506AV\#ABA & Serco CAS - 1 Generation Old & $x$ & $x$ & $x$ \\
\hline KIT, US RACKMOUNT, KEYBOARD MOUSE AND MONITOR & AG052A & Serco CAS - 1 Generation Old & $\mathrm{x}$ & $x$ & $\mathrm{x}$ \\
\hline KIT, US RACKMOUNT, KEYBOARD MOUSE AND MONITOR & TFT7600 & Serco CAS - Current & M & $x$ & $\mathrm{x}$ \\
\hline MEMORY KIT, DDR2 PC2300, 4GB $2 \times 2$ & 343057-B21 & Serco CAS - 3 Generations Old & $x$ & $x$ & $x$ \\
\hline MEMORY KIT, DDR2 PC2300, 4GB 2X2 & 343057-B21 & Serco CAS - 2 Generations Old & $x$ & $x$ & $\mathrm{x}$ \\
\hline MEMORY KIT, DDR2 PC2300, 4GB 2X2 & 343057-B21 & Serco CAS - 3 Generations Old & $x$ & $x$ & $x$ \\
\hline MEMORY KIT, DDR2 PC2300, 4GB $2 \times 2$ & 343057-B21 & Serco CAS - 2 Generations Old & $x$ & $x$ & $x$ \\
\hline MEMORY KIT, DDR2-400, 2GB 2X1 & DY288AV & Serco CAS - 3 Generations Old & $x$ & $x$ & $x$ \\
\hline MEMORY KIT, DDR2-400, 2GB 2X1 & DY288AV & Serco CAS - 3 Generations Old & $x$ & $x$ & $x$ \\
\hline MEMORY KIT, DDR2-667, 4GB 4X1 & GM568AV & Serco CAS - 1 Generation Old & $\mathrm{x}$ & $x$ & $x$ \\
\hline MEMORY KIT, DDR2-667, 4GB 4X1 & GM568AV & Serco CAS - 1 Generation Old & $x$ & $x$ & $x$ \\
\hline MEMORY KIT, DDR2-667, 4GB 4X1 & GM568AV & Serco CAS - 1 Generation Old & $x$ & $x$ & $x$ \\
\hline MEMORY KIT, DDR3-1333, 4GB 4X1 & & Serco CAS - Current & M & $x$ & $x$ \\
\hline MEMORY KIT, DDR3-1333, 4GB 4X1 & & Serco CAS - Current & M & $x$ & $x$ \\
\hline MEMORY KIT, DDR3-1333, 4GB 4X1 & & Serco CAS - Current & M & $x$ & $x$ \\
\hline MEMORY KIT, DDR3-1333, 8GB 1X8 & DL380G6 & Serco CAS - Current & $x$ & $x$ & $x$ \\
\hline MEMORY KIT, DDR3-1333, 8GB 1X8 & DL380G6 & Serco CAS - Current & $x$ & $x$ & $x$ \\
\hline MEMORY KIT, PC2-5300, 4GB 2X2 & 397413-B21 & Serco CAS - 1 Generation Old & $x$ & $x$ & $x$ \\
\hline MEMORY KIT, PC2-5300, 4GB 2X2 & 397413-B21 & Serco CAS - 1 Generation Old & $x$ & $x$ & $x$ \\
\hline MONITOR, FLAT PANEL, 17 INCH & L1706 & Serco CAS - 1 Generation Old & $x$ & $x$ & $x$ \\
\hline MONITOR, FLAT PANEL, 17 INCH & L1706 & Serco CAS - 2 Generations Old & $x$ & $x$ & $x$ \\
\hline MONITOR, FLAT PANEL, 17 INCH & L1706 & Serco CAS - 1 Generation Old & $x$ & $x$ & $x$ \\
\hline MONITOR, FLAT PANEL, 17 INCH & L1706 & Serco CAS - 3 Generations Old & $x$ & $x$ & $x$ \\
\hline MONITOR, FLAT PANEL, 17 INCH & PX849A8\#ABA & Serco CAS - 3 Generations Old & $x$ & $x$ & $x$ \\
\hline
\end{tabular}




\begin{tabular}{|c|c|c|c|c|c|}
\hline Nomenclature & Part Number & End Item & $\mathrm{SH}$ & $\mathrm{R}$ & 0 \\
\hline MONITOR, FLAT PANEL, 19 INCH & HPL 1910 & Serco CAS - Current & $x$ & $x$ & $x$ \\
\hline MONITOR, FLAT PANEL, 19 INCH & HPL 1910 & Serco CAS - Current & $\mathrm{x}$ & $x$ & $x$ \\
\hline MONITOR, FLAT PANEL, 19 INCH & L1906 & Serco CAS - 2 Generations Old & $\mathrm{x}$ & $\mathrm{x}$ & $\mathrm{x}$ \\
\hline MONITOR, FLAT PANEL, 19 INCH & L1906 & Serco CAS - 3 Generations Old & $x$ & $x$ & $x$ \\
\hline MONITOR, FLAT PANEL, 19 INCH & L1906 & Serco CAS - 1 Generation Old & $\mathrm{x}$ & $\mathrm{x}$ & $\mathrm{x}$ \\
\hline MONITOR, FLAT PANEL, 20 INCH & EF227A4\#ABA & Serco CAS - 1 Generation Old & $\mathrm{x}$ & $\mathrm{x}$ & $\mathrm{x}$ \\
\hline MONITOR, FLAT PANEL, 20 INCH & EF227A4\#ABA & Serco CAS - 1 Generation Old & $\mathrm{x}$ & $x$ & $x$ \\
\hline MONITOR, FLAT PANEL, 20 INCH & EF227A4\#ABA & Serco CAS - 2 Generations Old & $x$ & $\mathrm{x}$ & $x$ \\
\hline MONITOR, FLAT PANEL, 20 INCH & EF227A4\#ABA & Serco CAS - 1 Generation Old & $x$ & $x$ & $x$ \\
\hline MONITOR, FLAT PANEL, 20 INCH & LP2065 & Serco CAS - Current & $\mathrm{x}$ & $\mathrm{x}$ & $\mathrm{x}$ \\
\hline MONITOR, FLAT PANEL, 20 INCH & LP2065 & Serco CAS - Current & $\mathrm{x}$ & $x$ & $x$ \\
\hline MONITOR, FLAT PANEL, 20 INCH & LP2065 & Serco CAS - Current & $x$ & $x$ & $x$ \\
\hline MOUSE, PS2 & AA360AV & Serco CAS - 2 Generations Old & $x$ & $x$ & $x$ \\
\hline MOUSE, PS2 & AA360AV & Serco CAS - 3 Generations Old & $x$ & $x$ & $x$ \\
\hline MOUSE, PS2 & AA360AV & Serco CAS - 2 Generations Old & $\mathrm{x}$ & $\mathrm{x}$ & $\mathrm{x}$ \\
\hline MOUSE, PS2 & GM598AV & Serco CAS - 1 Generation Old & $x$ & $x$ & $x$ \\
\hline MOUSE, PS2 & GM598AV & Serco CAS - 1 Generation Old & $\mathrm{x}$ & $x$ & $x$ \\
\hline MOUSE, PS2 & GM598AV & Serco CAS - 1 Generation Old & $x$ & $\mathrm{x}$ & $\mathrm{x}$ \\
\hline MOUSE, PS2, OPTICAL & & Serco CAS - Current & $\mathrm{x}$ & $\mathrm{x}$ & $\mathrm{x}$ \\
\hline MOUSE, PS2, OPTICAL & & Serco CAS - Current & $x$ & $x$ & $x$ \\
\hline MOUSE, PS2, OPTICAL & & Serco CAS - Current & $x$ & $x$ & $x$ \\
\hline NETWORK CARD, PCIE, DUAL PORT GIGABIT & 394795-B21 & Serco CAS - 1 Generation Old & $x$ & $x$ & $x$ \\
\hline NETWORK CARD, PCIE, DUAL PORT GIGABIT & 394795-B21 & Serco CAS - 1 Generation Old & $x$ & $x$ & $x$ \\
\hline NETWORK CARD, PCIE, DUAL PORT GIGABIT & DL380G6 & Serco CAS - Current & $x$ & $\mathrm{x}$ & $\mathrm{x}$ \\
\hline NETWORK CARD, PCIE, DUAL PORT GIGABIT & DL380G6 & Serco CAS - Current & $\mathrm{x}$ & $\mathrm{x}$ & $\mathrm{x}$ \\
\hline
\end{tabular}




\begin{tabular}{|c|c|c|c|c|c|}
\hline Nomenclature & Part Number & End Item & $\mathrm{SH}$ & $\mathrm{R}$ & $\mathrm{O}$ \\
\hline NETWORK CARD, PCIE, GIGABIT & & Serco CAS - Current & $x$ & $x$ & $x$ \\
\hline NETWORK CARD, PCI-X, GIGABIT & 290563-B21 & Serco CAS - 2 Generations Old & $x$ & $x$ & $\mathrm{x}$ \\
\hline NETWORK CARD, PCI-X, GIGABIT & 290563-B21 & Serco CAS - 2 Generations Old & $x$ & $x$ & $x$ \\
\hline NETWORK CARD, PCI-X, GIGABIT & 290563-B21 & Serco CAS - 3 Generations Old & $x$ & $x$ & $\mathrm{x}$ \\
\hline NETWORK CARD, PCI-X, GIGABIT & 290563-B21 & Serco CAS - 3 Generations Old & $x$ & $x$ & $x$ \\
\hline OPTICAL DRIVE, DVDROM CDR & ER272AV & Serco CAS - 2 Generations Old & $x$ & $\mathrm{x}$ & $x$ \\
\hline OPTICAL DRIVE, DVDROM CDR & ER272AV & Serco CAS - 2 Generations Old & $x$ & $x$ & $x$ \\
\hline OPTICAL DRIVE, DVDROM CDRW, $48 \mathrm{X}$ & DY295AV & Serco CAS - 3 Generations Old & $\mathrm{x}$ & $x$ & $x$ \\
\hline OPTICAL DRIVE, DVDROM CDRW, $48 \mathrm{X}$ & DY295AV & Serco CAS - 3 Generations Old & $x$ & $x$ & $x$ \\
\hline OPTICAL DRIVE, SATA, 16X DVD-ROM & & Serco CAS - Current & $x$ & $x$ & $x$ \\
\hline OPTICAL DRIVE, SATA, 16X DVD-ROM & & Serco CAS - Current & $x$ & $x$ & $x$ \\
\hline OPTICAL DRIVE, SATA, 16X DVD-ROM & & Serco CAS - Current & $x$ & $x$ & $x$ \\
\hline OPTICAL DRIVE, SATA, 16X DVD-ROM & GM577AV & Serco CAS - 1 Generation Old & $x$ & $x$ & $x$ \\
\hline OPTICAL DRIVE, SATA, 16X DVD-ROM & GM577AV & Serco CAS - 1 Generation Old & $\mathrm{x}$ & $\mathrm{x}$ & $x$ \\
\hline OPTICAL DRIVE, SATA, 16X DVD-ROM & GM577AV & Serco CAS - 1 Generation Old & $\mathrm{x}$ & $x$ & $x$ \\
\hline OPTICAL DRIVE, SATA, 8X 24X DVD-ROM & 264007-B21 & Serco CAS - 1 Generation Old & $x$ & $x$ & $x$ \\
\hline OPTICAL DRIVE, SATA, 8X 24X DVD-ROM & 264007-B21 & Serco CAS - 3 Generations Old & $x$ & $x$ & $x$ \\
\hline OPTICAL DRIVE, SATA, 8X 24X DVD-ROM & 264007-B21 & Serco CAS - 2 Generations Old & $\mathrm{x}$ & $x$ & $x$ \\
\hline OPTICAL DRIVE, SATA, 8X 24X DVD-ROM & 264007-B21 & Serco CAS - 3 Generations Old & $x$ & $x$ & $x$ \\
\hline OPTICAL DRIVE, SATA, 8X 24X DVD-ROM & 264007-B21 & Serco CAS - 1 Generation Old & $x$ & $x$ & $x$ \\
\hline OPTICAL DRIVE, SATA, DVD-ROM CD-R & 264007-B21 & Serco CAS - 2 Generations Old & $x$ & $x$ & $x$ \\
\hline OPTICAL DRIVE, SATA, SLIM DVD-ROM & DL380G6 & Serco CAS - Current & $\mathrm{x}$ & $x$ & $x$ \\
\hline OPTICAL DRIVE, SATA, SLIM DVD-ROM & DL380G6 & Serco CAS - Current & $x$ & $x$ & $x$ \\
\hline POWER SUPPLY, 350/370/380 & 399771-B21 & Serco CAS - 1 Generation Old & $x$ & $x$ & $x$ \\
\hline POWER SUPPLY, 350/370/380 & 399771-B21 & Serco CAS - 1 Generation Old & $x$ & $x$ & $x$ \\
\hline POWER SUPPLY, AC POWER, 460W & DL380G6 & Serco CAS - Current & $x$ & $x$ & $x$ \\
\hline POWER SUPPLY, AC POWER, 460W & DL380G6 & Serco CAS - Current & $\mathrm{x}$ & $x$ & $x$ \\
\hline POWER SUPPLY, AC REDUNDANT, HOT PLUG & $355892-001$ & Serco CAS - 3 Generations Old & $x$ & $x$ & $x$ \\
\hline POWER SUPPLY, AC REDUNDANT, HOT PLUG & 355892-001 & Serco CAS - 3 Generations Old & $x$ & $x$ & $\mathrm{X}$ \\
\hline
\end{tabular}




\begin{tabular}{|c|c|c|c|c|c|}
\hline Nomenclature & Part Number & End Item & $\mathrm{SH}$ & $\mathrm{R}$ & $\mathrm{O}$ \\
\hline POWER SUPPLY, AC REDUNDANT, HOT PLUG & $355892-001$ & Serco CAS - 2 Generations Old & $\mathrm{x}$ & $\mathrm{x}$ & $\mathrm{x}$ \\
\hline POWER SUPPLY, AC REDUNDANT, HOT PLUG & $355892-001$ & Serco CAS - 2 Generations Old & $x$ & $x$ & $x$ \\
\hline PRINTER, WITH SCANNER AND FAX & & SafePass CAS & $x$ & $\mathrm{x}$ & $x$ \\
\hline SERVER, 1 CPU 4GB RAM, 160GB & & SafePass CAS & M & $x$ & $x$ \\
\hline SERVER, 1 CPU 4GB RAM, 250GB & & SafePass CAS & M & $x$ & $x$ \\
\hline SERVER, 1 CPU 4GB RAM, 5X300 GB & & SafePass CAS & M & $x$ & $x$ \\
\hline SERVER, 2 CPU 4GB RAM, 5X300 GB & & SafePass CAS & M & $\mathrm{x}$ & $\mathrm{x}$ \\
\hline SERVER, PROLIANT & DL380 G4 & ICIS OCR & $x$ & $x$ & $x$ \\
\hline SOFTWARE, DOWNGRADE TO XP 32 BIT & & Serco CAS - Current & $x$ & $x$ & $\mathrm{x}$ \\
\hline SOFTWARE, DOWNGRADE TO XP 32 BIT & & Serco CAS - Current & $x$ & $x$ & $x$ \\
\hline SOFTWARE, DOWNGRADE TO XP 32 BIT & & Serco CAS - Current & $x$ & $x$ & $x$ \\
\hline SOFTWARE, DOWNGRADE TO XP 32 BIT & GR528AV\#ABA & Serco CAS - 1 Generation Old & $x$ & $x$ & $x$ \\
\hline SOFTWARE, DOWNGRADE TO XP 32 BIT & GR528AV\#ABA & Serco CAS - 1 Generation Old & $x$ & $x$ & $x$ \\
\hline SOFTWARE, DOWNGRADE TO XP 32 BIT & GR528AV\#ABA & Serco CAS - 1 Generation Old & $x$ & $x$ & $x$ \\
\hline SPEAKER, SOUND BAR & & Serco CAS - Current & $x$ & $x$ & $x$ \\
\hline SPEAKER, SOUND BAR & & Serco CAS - Current & $x$ & $x$ & $x$ \\
\hline SPEAKER, SOUND BAR & & Serco CAS - Current & $x$ & $x$ & $x$ \\
\hline SPEAKER, SOUND BAR & EE418AA & Serco CAS - 2 Generations Old & $x$ & $x$ & $x$ \\
\hline SPEAKER, SOUND BAR & EE418AA & Serco CAS - 1 Generation Old & $x$ & $x$ & $x$ \\
\hline SPEAKER, SOUND BAR & EE418AA & Serco CAS - 1 Generation Old & $x$ & $x$ & $x$ \\
\hline SPEAKER, SOUND BAR & EE418AA & Serco CAS - 1 Generation Old & $x$ & $x$ & $x$ \\
\hline SWITCH, CAT5 KVM, 0X1X8 & & Serco CAS - Current & $x$ & $x$ & $x$ \\
\hline SWITCH, CAT5 KVM, 0X2X16 & 336045-B21 & Serco CAS - 1 Generation Old & $x$ & $x$ & $x$ \\
\hline SWITCH, SAN, 4/8 BASE & A7984A & Serco CAS - 1 Generation Old & $x$ & $x$ & $x$ \\
\hline TAPE DRIVE, MSL & BL531A & Serco CAS - 1 Generation Old & M & $x$ & $x$ \\
\hline TAPE DRIVE, MSL & BL531A & Serco CAS - 2 Generations Old & M & $x$ & $x$ \\
\hline TAPE DRIVE, MSL & BL531A & Serco CAS - 3 Generations Old & M & $x$ & $x$ \\
\hline TAPE DRIVE, MSL & MSL 2024 & Serco CAS - Current & M & $x$ & $x$ \\
\hline VIDEO CARD, NVIDIA QUADRO, 128MB & EE058AV & Serco CAS - 3 Generations Old & $x$ & $x$ & $\mathrm{x}$ \\
\hline
\end{tabular}




\begin{tabular}{|c|c|c|c|c|c|}
\hline Nomenclature & Part Number & End Item & $\mathrm{SH}$ & $\mathrm{R}$ & $\mathrm{O}$ \\
\hline VIDEO CARD, NVIDIA QUADRO, 128MB & EE058AV & Serco CAS - 3 Generations Old & $\mathrm{x}$ & $\mathrm{x}$ & $\mathrm{x}$ \\
\hline VIDEO CARD, NVIDIA QUADRO, 128MB & RD064AV & Serco CAS - 2 Generations Old & $x$ & $\mathrm{x}$ & $x$ \\
\hline VIDEO CARD, NVIDIA QUADRO, 128MB & RD064AV & Serco CAS - 2 Generations Old & $\mathrm{x}$ & $\mathrm{x}$ & $\mathrm{x}$ \\
\hline VIDEO CARD, NVIDIA QUADRO, 256MB & GN493AV & Serco CAS - 1 Generation Old & $\mathrm{x}$ & $\mathrm{x}$ & $\mathrm{x}$ \\
\hline VIDEO CARD, NVIDIA QUADRO, 256MB & GN493AV & Serco CAS - 1 Generation Old & $\mathrm{x}$ & $\mathrm{x}$ & $\mathrm{x}$ \\
\hline VIDEO CARD, NVIDIA QUADRO, 256MB & GN493AV & Serco CAS - 1 Generation Old & $\mathrm{x}$ & $\mathrm{x}$ & $\mathrm{x}$ \\
\hline VIDEO CARD, NVIDIA QUADRO, 256MB & NVS295 & Serco CAS - Current & $x$ & $x$ & $x$ \\
\hline VIDEO CARD, NVIDIA QUADRO, 256MB & NVS295 & Serco CAS - Current & $\mathrm{x}$ & $x$ & $x$ \\
\hline VIDEO CARD, NVIDIA QUADRO, 256MB & NVS295 & Serco CAS - Current & $x$ & $x$ & $\mathrm{x}$ \\
\hline WORK STATION & & Serco CAS - Current & M & $\mathrm{x}$ & $\mathrm{x}$ \\
\hline WORK STATION & & Serco CAS - Current & M & $\mathrm{x}$ & $\mathrm{x}$ \\
\hline WORK STATION & & Serco CAS - Current & M & $x$ & $x$ \\
\hline WORK STATION & DU937AV & Serco CAS - 3 Generations Old & $\mathrm{x}$ & $x$ & $\mathrm{x}$ \\
\hline WORK STATION & DU937AV & Serco CAS - 3 Generations Old & $\mathrm{x}$ & $\mathrm{x}$ & $\mathrm{x}$ \\
\hline WORK STATION & ER236AV & Serco CAS - 2 Generations Old & $x$ & $\mathrm{x}$ & $x$ \\
\hline WORK STATION & ER236AV & Serco CAS - 2 Generations Old & $x$ & $x$ & $x$ \\
\hline WORK STATION & RV725AV & Serco CAS - 1 Generation Old & $\mathrm{x}$ & $\mathrm{x}$ & $\mathrm{x}$ \\
\hline WORK STATION & RV725AV & Serco CAS - 1 Generation Old & $x$ & $x$ & $x$ \\
\hline WORK STATION & RV725AV & Serco CAS - 1 Generation Old & $\mathrm{x}$ & $\mathrm{x}$ & $\mathrm{x}$ \\
\hline \multicolumn{6}{|l|}{ Hitachi } \\
\hline $\mathrm{HDD}, 40 \mathrm{~GB}$ & & RAVEN CAS & $\mathrm{x}$ & $\mathrm{x}$ & $\mathrm{x}$ \\
\hline \multicolumn{6}{|l|}{ Hoffman } \\
\hline ENCLOSURE, REPEATER, MACHINED & $7049 R$ & RAVEN CAS & $x$ & $x$ & $x$ \\
\hline LENS, HOFFMAN FIXTURE & 87576853 & Utility Panels & $\mathrm{x}$ & $\mathrm{x}$ & $\mathrm{x}$ \\
\hline PANEL, 8X6 & $7055 \mathrm{P}$ & RAVEN CAS & $\mathrm{x}$ & $x$ & $\mathrm{x}$ \\
\hline SEAL, 2 INCH HOLE & 59760 & Utility Panels & $\mathrm{x}$ & $\mathrm{x}$ & $\mathrm{x}$ \\
\hline UTILITY BOX, 8X6X4 & $7055 \mathrm{~B}$ & RAVEN CAS & $\mathrm{x}$ & $\mathrm{x}$ & $\mathrm{x}$ \\
\hline
\end{tabular}




\begin{tabular}{|c|c|c|c|c|c|}
\hline Nomenclature & Part Number & End Item & $\mathrm{SH}$ & $\mathrm{R}$ & O \\
\hline LIMIT SWITCH, PIN HEAD WITH ROLLER LEVER, SPDT & $B Z-2 R L 2-A 2$ & Utility Panels & $x$ & $x$ & $x$ \\
\hline \multicolumn{6}{|l|}{ HTS } \\
\hline CABLE, SMB, MALE & 550054 & HTS OCR & $x$ & $x$ & $x$ \\
\hline CONNECTOR, BCN, MALE TO DOUBLE & 509325 & HTS OCR & $x$ & $x$ & $\mathrm{x}$ \\
\hline DETECTOR, LOOP, MATRIX & 545010 & HTS OCR & $\mathrm{x}$ & $x$ & $\mathrm{x}$ \\
\hline DETECTOR, LOOP, SOCKET MATRIX & 545011 & HTS OCR & $x$ & $x$ & $x$ \\
\hline FRAME GRABBER & 560217 & HTS OCR & M & $\mathrm{x}$ & $\mathrm{x}$ \\
\hline IO CARD & 550117 & HTS OCR & $x$ & $x$ & $x$ \\
\hline LICENSE, USB PLUG & 580044 & HTS OCR & $\mathrm{x}$ & $\mathrm{x}$ & $x$ \\
\hline RECEIVER & 547008 & HTS OCR & $x$ & $x$ & $x$ \\
\hline SERVER, PENTIUM 4 & 940000 & HTS OCR & M & $x$ & $x$ \\
\hline SET, TERMINAL BOARD & 580136 & HTS OCR & $x$ & $\mathrm{x}$ & $\mathrm{x}$ \\
\hline SOFTWARE, RELEASE CD & 550045 & HTS OCR & $x$ & $x$ & $x$ \\
\hline SYNCHRONIZATION GENERATOR & 910373 & HTS OCR & $x$ & $x$ & $x$ \\
\hline TRANSMITTER & 547007 & HTS OCR & $x$ & $x$ & $x$ \\
\hline VIDEO DISTRIBUTER & 910371 & HTS OCR & $x$ & $x$ & $x$ \\
\hline \multicolumn{6}{|l|}{ Hummel } \\
\hline GRIP, CORD, $1 / 2$ & 7305D & RAVEN CAS & $x$ & $x$ & $x$ \\
\hline GRIP, CORD, $1 / 4$ & $7305 \mathrm{G}$ & RAVEN CAS & $x$ & $x$ & $x$ \\
\hline GRIP, CORD, 3/4 & $7305 \mathrm{~F}$ & RAVEN CAS & $x$ & $x$ & $x$ \\
\hline \multicolumn{6}{|l|}{ IBase Technology } \\
\hline CONCENTRATOR & $0463 \mathrm{~A}$ & RAVEN CAS & $x$ & $x$ & $x$ \\
\hline \multicolumn{6}{|l|}{ ICIS } \\
\hline CABLE, FC SC SM, 3M & FCSC-SMD-02 & ICIS OCR & $x$ & $\mathrm{x}$ & $x$ \\
\hline CABLE, LC SC SM, 2M & FCSC-SMD-02 & ICIS OCR & $x$ & $x$ & $x$ \\
\hline CABLE, LC SC SM, 3M & 13-HPS108-3M & ICIS OCR & $x$ & $x$ & $x$ \\
\hline CABLE, SC SC SM, 5M & SCSSTG16 & ICIS OCR & $x$ & $x$ & $x$ \\
\hline RECEIVER, VIDEO, SM RX & $431489-002$ & ICIS OCR & $x$ & $x$ & $x$ \\
\hline
\end{tabular}




\begin{tabular}{|c|c|c|c|c|c|}
\hline Nomenclature & Part Number & End Item & $\mathrm{SH}$ & $\mathrm{R}$ & $\mathrm{O}$ \\
\hline SERVER, ICIS APP & 431413-001 & ICIS OCR & $\mathrm{x}$ & $x$ & $x$ \\
\hline SWITCH TRANSCEIVER, GIGABIT EHTERNET, SFP & $421063-001$ & ICIS OCR & $x$ & $x$ & $x$ \\
\hline \multicolumn{6}{|l|}{ ISC Sales } \\
\hline AIR CONDITIONER & NE03012604BXO & Serco Wireless Network & M & $x$ & $\mathrm{x}$ \\
\hline \multicolumn{6}{|l|}{ Leviton } \\
\hline CABLE, CAT6 PLUS, 7 FT & $6246007 L$ & Siemens Wireless Network & $x$ & $x$ & $x$ \\
\hline CABLE, FIBER OPTIC, 1M & Cat\#62DSC-M01 & Utility Panels & $\mathrm{x}$ & $x$ & $\mathrm{x}$ \\
\hline CABLE, FIBER OPTIC, 3M & Cat\#62DSC-M03 & Utility Panels & $\mathrm{x}$ & $\mathrm{x}$ & $x$ \\
\hline POWER SUPPLY & $12 \mathrm{~A}$ & Utility Panels & $x$ & $x$ & $\mathrm{x}$ \\
\hline \multicolumn{6}{|l|}{ Lumenera } \\
\hline CAMERA, NETWORK, COLOR 1.4MP MEGA RESOLUTION & & Siemens OCR & M & $x$ & $x$ \\
\hline \multicolumn{6}{|l|}{ Magnetic Autocontrol } \\
\hline ACCESSORY, TRAFFIC ARM & $3004-0001$ & Traffic Arms & $\mathrm{x}$ & $\mathrm{x}$ & $x$ \\
\hline BOOM , 12 FT, SWING AWAY & MSB-035 & Traffic Arms & $x$ & $x$ & $x$ \\
\hline BOOM , 15 FT & MSB 5N-045 & Traffic Arms & $x$ & $x$ & $x$ \\
\hline CONTROLLER, MLC DROP BAR & MLC & Traffic Arms & $x$ & $x$ & $x$ \\
\hline KIT, BARRIER ARM ATTACHMENT & $1031-0180$ & Traffic Arms & $\mathrm{x}$ & $\mathrm{x}$ & $\mathrm{x}$ \\
\hline MOTOR, DRIVE, MIB-30 230V & MIB 30-C920 & Traffic Arms & M & $x$ & $x$ \\
\hline MOTOR, DRIVE, MIB-40 240V & MIB 40-C920 & Traffic Arms & M & $x$ & $x$ \\
\hline SET, SWING AWAY FLANGE & $1031-5052$ & Traffic Arms & $x$ & $x$ & $x$ \\
\hline WIRE, INDUCTION LOOP & 90000.0001 & Traffic Arms & $x$ & $x$ & $x$ \\
\hline \multicolumn{6}{|l|}{ Mean Well } \\
\hline CONVERTER, 12 VDC TO 5 VDC & $7499 B$ & RAVEN CAS & $x$ & $x$ & $x$ \\
\hline INVERTER, POWER & TS200-124A & Schneider CAS & $x$ & $x$ & $x$ \\
\hline POWER SUPPLY, 7.5A 13.5 VDC & $7499 C$ & RAVEN CAS & $x$ & $x$ & $x$ \\
\hline UPS & DR-UPS-40 & Schneider CAS & $\mathrm{H}$ & $x$ & $\mathrm{x}$ \\
\hline \multicolumn{6}{|l|}{ Microsoft } \\
\hline KIT, KEYBOARD MOUSE & & SafePass CAS & $\mathrm{x}$ & $\mathrm{x}$ & $x$ \\
\hline
\end{tabular}




\begin{tabular}{|c|c|c|c|c|c|}
\hline Nomenclature & Part Number & End Item & $\mathrm{SH}$ & $\mathrm{R}$ & 0 \\
\hline SOFTWARE, 5 CAL & & SafePass CAS & $x$ & $x$ & $x$ \\
\hline SOFTWARE, 5 CAL & & SafePass CAS & M & $x$ & $x$ \\
\hline \multicolumn{6}{|l|}{ Minute Man } \\
\hline UPS, 2300 VA & 0475 & RAVEN CAS & $\mathrm{MH}$ & $x$ & $x$ \\
\hline \multicolumn{6}{|l|}{$\mathrm{MW}$} \\
\hline CONVERTER, 48 VDC & $C D(S D)-25 C-24$ & Serco Wireless Network & $x$ & $x$ & $x$ \\
\hline POWER SUPPLY, 48 V AC & RB17137153 & Serco Wireless Network & M & $x$ & $x$ \\
\hline \multicolumn{6}{|l|}{ NEC } \\
\hline MONITOR, FLAT PANEL, 17 INCH & & SafePass CAS & $x$ & $x$ & $x$ \\
\hline \multicolumn{6}{|l|}{ Netgear } \\
\hline SWITCH, 16 PORT, ETHERNET & $0464 G$ & RAVEN CAS & $x$ & $x$ & $x$ \\
\hline SWITCH, 8 PORT & & SafePass CAS & $x$ & $x$ & $x$ \\
\hline SWITCH, RACK MOUNT, 12 PORT & & SafePass CAS & M & $x$ & $x$ \\
\hline \multicolumn{6}{|l|}{ Norton } \\
\hline SOFTWARE, BACKUP, GHOST & & SafePass CAS & $x$ & $x$ & $x$ \\
\hline \multicolumn{6}{|l|}{ PC CITY BUILD } \\
\hline WORK STATION, RAVEN & 0458 & RAVEN CAS & M & $x$ & 0 \\
\hline \multicolumn{6}{|l|}{ Pelco } \\
\hline ADAPTER, POLE & 0461B & RAVEN CAS & $x$ & $x$ & $x$ \\
\hline ADAPTER, POLE & PA101 & Schneider CAS & $x$ & $\mathrm{x}$ & $x$ \\
\hline BRACKET, CAMERA, POLE MOUNT & EM2000 & Siemens Wireless Network & $x$ & $x$ & $x$ \\
\hline DEFROSTER, 230 VAC & $0461 \mathrm{G}$ & RAVEN CAS & $x$ & $x$ & $x$ \\
\hline ENCLOSURE, ENVIRONMENTAL & & Schneider CAS & $x$ & $x$ & $x$ \\
\hline ENCLOSURE, ENVIRONMENTAL, HEATER AND BLOWER & $\mathrm{EH} 4718-2$ & Schneider CAS & $x$ & $x$ & $x$ \\
\hline HOUSING, CAMERA, 230 VAC & $0461 \mathrm{~F}$ & RAVEN CAS & $x$ & $x$ & $x$ \\
\hline HOUSING, CAMERA, 24 VAC & $0461 \mathrm{E}$ & RAVEN CAS & $x$ & $x$ & $x$ \\
\hline HOUSING, CAMERA, OUTDOOR & EH4718-3 & Siemens Wireless Network & $x$ & $x$ & $x$ \\
\hline LENS, IR CORRECTED AI DIRECT DRIVE, $1 / 3$ INCH 2.8 TO $11 \mathrm{MM}$ & 13VDIR2.8-1 1 & Schneider CAS & $x$ & $x$ & $x$ \\
\hline
\end{tabular}




\begin{tabular}{|c|c|c|c|c|c|}
\hline Nomenclature & Part Number & End Item & $\mathrm{SH}$ & $\mathrm{R}$ & $\mathrm{O}$ \\
\hline LENS, IR CORRECTED AI DIRECT DRIVE, 1/3 INCH 3 TO 8.5MM & 13VDIR3-8.5 & Schneider CAS & $x$ & $x$ & $x$ \\
\hline MOUNT, WALL & $0461 C$ & RAVEN CAS & $\mathrm{x}$ & $x$ & $x$ \\
\hline MOUNT, WALL & EM22 & Schneider CAS & $x$ & $x$ & $x$ \\
\hline POWER SUPPLY, 4A, 1 OUTPUT & WCS1-4 & Schneider CAS & $x$ & $x$ & $x$ \\
\hline POWER SUPPLY, CAMERA & 0462 & RAVEN CAS & $x$ & $x$ & $x$ \\
\hline POWER SUPPLY, CAMERA & 0462A & RAVEN CAS & $x$ & $x$ & $x$ \\
\hline POWER SUPPLY, CAMERA & 0462B & RAVEN CAS & $x$ & $x$ & $x$ \\
\hline SUN SHIELD & $0461 \mathrm{H}$ & RAVEN CAS & $x$ & $x$ & $x$ \\
\hline SUN SHIELD & SS4718 & Schneider CAS & $x$ & $x$ & $x$ \\
\hline SUN SHIELD, CAMERA & SS4718 & Siemens Wireless Network & $x$ & $x$ & $x$ \\
\hline \multicolumn{6}{|l|}{ Pentax } \\
\hline LENS, CAMERA, OBJECTIVE 1.6MM & C1614A & Siemens OCR & $x$ & $x$ & $x$ \\
\hline LENS, CAMERA, OBJECTIVE 25MM & B2514D & Siemens OCR & $x$ & $x$ & $x$ \\
\hline LENS, CAMERA, OBJECTIVE 4.8MM & C418DX & Siemens OCR & $x$ & $x$ & $x$ \\
\hline \multicolumn{6}{|l|}{ Phillips } \\
\hline LAMP & F15T8/CW/ALTO & Utility Panels & $x$ & $x$ & $x$ \\
\hline \multicolumn{6}{|l|}{ Phoenix Contact } \\
\hline RELAY, AUTOMATION DIRECT & PLC-RSC-2300 & Utility Panels & $x$ & $x$ & $x$ \\
\hline TERMINAL, FUSIBLE, WITH LED & UT 6-HESI & Utility Panels & $x$ & $x$ & $x$ \\
\hline \multicolumn{6}{|l|}{ PI } \\
\hline CABLE, POWER, IEC MALE TO FEMALE & $7303 B$ & RAVEN CAS & $x$ & $x$ & $x$ \\
\hline \multicolumn{6}{|l|}{ Prolink } \\
\hline CABLE, CAT6, 2.13M & R5032XGN & Siemens Wireless Network & $x$ & $x$ & $x$ \\
\hline \multicolumn{6}{|l|}{ Quabbin Wire \& Cable } \\
\hline CABLE, 12 PAIR CROSSOVER, $1000 \mathrm{FT}$ & 8525 & RAVEN CAS & M & $x$ & $x$ \\
\hline \multicolumn{6}{|l|}{ Raytec } \\
\hline ILLUMINATOR, IR & RM25-30 & Schneider CAS & $x$ & $x$ & $x$ \\
\hline ILLUMINATOR, IR & RM100-A1-50 & Schneider CAS & $x$ & $x$ & $x$ \\
\hline
\end{tabular}




\begin{tabular}{|c|c|c|c|c|c|}
\hline Nomenclature & Part Number & End Item & $\mathrm{SH}$ & $\mathrm{R}$ & $\mathrm{O}$ \\
\hline ILLUMINATOR, LED, 50 & & Siemens OCR & $x$ & $x$ & $x$ \\
\hline \multicolumn{6}{|l|}{ Rittal } \\
\hline CABINET, TS NEMA 4 & TS 8886640 & Serco Wireless Network & M & $\mathrm{x}$ & $x$ \\
\hline CABINET, TS NEMA 4 & TS 8886641 & Serco Wireless Network & M & $\mathrm{x}$ & $\mathrm{x}$ \\
\hline SHELF & 7185035 & Serco Wireless Network & $\mathrm{x}$ & $\mathrm{x}$ & $x$ \\
\hline \multicolumn{6}{|l|}{ Sarix } \\
\hline CAMERA, NETWORK & AAM-CROO & Schneider CAS & M & $\mathrm{x}$ & $\mathrm{x}$ \\
\hline \multicolumn{6}{|l|}{ Scaleance } \\
\hline RADIO, WIRELESS & OAA60 & Siemens Wireless Network & $x$ & $\mathrm{x}$ & $x$ \\
\hline RADIO, WIRELESS & OAB60 & Siemens Wireless Network & $\mathrm{x}$ & $\mathrm{x}$ & $\mathrm{x}$ \\
\hline SWITCH, SCALEANCE & $\mathrm{x} 101-1$ & Siemens Wireless Network & $\mathrm{x}$ & $\mathrm{x}$ & $\mathrm{x}$ \\
\hline SWITCH, SCALEANCE & X308 2LD & Siemens Wireless Network & M & $\mathrm{x}$ & $x$ \\
\hline SWITCH, SCALEANCE & X310 FE & Siemens Wireless Network & $\mathrm{x}$ & $\mathrm{x}$ & $\mathrm{x}$ \\
\hline SWITCH, SCALEANCE & $x 408-2$ & Siemens Wireless Network & M & $x$ & $x$ \\
\hline SWITCH, SCALEANCE & X414 3E & Siemens Wireless Network & $x$ & $x$ & $x$ \\
\hline \multicolumn{6}{|l|}{ Seven Star } \\
\hline POWER STRIP, UNIVERSAL & $7303 P$ & RAVEN CAS & $\mathrm{x}$ & $\mathrm{x}$ & $\mathrm{x}$ \\
\hline \multicolumn{6}{|l|}{ Sick } \\
\hline SENSOR, OPTICAL PAIR, PAIR SEND AND RECEIVE & & Siemens OCR & $x$ & $x$ & $x$ \\
\hline \multicolumn{6}{|l|}{ Siemens } \\
\hline ANTENNA TERMINATOR, $50 \mathrm{OHM}$ & TI 795-1R & Siemens Wireless Network & $x$ & $\mathrm{x}$ & $x$ \\
\hline CIRCUIT BREAKER, 6KA1 N POL, C.5 & & Siemens OCR & $\mathrm{x}$ & $\mathrm{x}$ & $\mathrm{x}$ \\
\hline CIRCUIT BREAKER, 6KA1 N POL, C1 & & Siemens OCR & $x$ & $x$ & $x$ \\
\hline CIRCUIT BREAKER, 6KA1 N POL, C10 & & Siemens OCR & $x$ & $\mathrm{x}$ & $x$ \\
\hline CIRCUIT BREAKER, 6KA1 N POL, C3 & & Siemens OCR & $x$ & $x$ & $x$ \\
\hline CIRCUIT BREAKER, 6KA1 N POL, C6 & & Siemens OCR & $x$ & $x$ & $x$ \\
\hline CONFIGURATION PLUG & 6GK1900-0АВ00 & Siemens Wireless Network & $x$ & $x$ & $x$ \\
\hline EXTENDER MODULE & EM495-8 & Siemens Wireless Network & $\mathrm{x}$ & $\mathrm{x}$ & $\mathrm{x}$ \\
\hline
\end{tabular}




\begin{tabular}{|c|c|c|c|c|c|}
\hline Nomenclature & Part Number & End Item & $\mathrm{SH}$ & $\mathrm{R}$ & $\mathrm{O}$ \\
\hline MEDIA MODULE & MM491-2 & Siemens Wireless Network & $\mathrm{x}$ & $\mathrm{x}$ & $\mathrm{x}$ \\
\hline MEDIA MODULE & MM492-2LD & Siemens Wireless Network & M & $\mathrm{x}$ & $\mathrm{x}$ \\
\hline POWER SUPPLY, 2AC & PS791 & Siemens Wireless Network & $x$ & $x$ & $x$ \\
\hline VOLTAGE PROTECTOR, 230V & & Siemens OCR & $x$ & $x$ & $x$ \\
\hline VOLTAGE PROTECTOR, CAT6 & & Siemens OCR & $\mathrm{x}$ & $\mathrm{x}$ & $\mathrm{x}$ \\
\hline \multicolumn{6}{|l|}{ Simatic } \\
\hline RACK, PC & & Siemens OCR & M & $x$ & $x$ \\
\hline \multicolumn{6}{|l|}{ Sitop } \\
\hline POWER SUPPLY, 2.5A 24V & 6ЕР1333-2BA01 & Siemens Wireless Network & $x$ & $x$ & $x$ \\
\hline \multicolumn{6}{|l|}{ SoftwareFX } \\
\hline SOFTWARE, WINDOWS FORMS & & SafePass CAS & M & $x$ & $x$ \\
\hline \multicolumn{6}{|l|}{ SOLA } \\
\hline UPS, DIN RAIL MOUNTABLE & SDU 850 & Serco Wireless Network & $\mathrm{H}$ & $x$ & $x$ \\
\hline \multicolumn{6}{|l|}{ Sony } \\
\hline CAMERA & 0460 & RAVEN CAS & M & $x$ & $\mathrm{O}$ \\
\hline CAMERA, DIGITAL, SURVEILLANCE & SNC-Z20N & Siemens Wireless Network & $x$ & $x$ & 0 \\
\hline CAMERA, REAR & 17COLREARO6ASSY & HTS OCR & $x$ & $x$ & $x$ \\
\hline CAMERA, SCH-2, YELLOW & 19BWOR3712500EL & HTS OCR & M & $x$ & $x$ \\
\hline CAMERA, SIDE & 17COL000440ASSY & HTS OCR & $x$ & $x$ & $x$ \\
\hline CAMERA, TOP & 17COLTOP044ASSY & HTS OCR & $x$ & $x$ & $x$ \\
\hline \multicolumn{6}{|l|}{ SORENSEN } \\
\hline POWER SUPPLY, 48 VDC & XFR60-20M13A & Serco Wireless Network & $M$ & $x$ & 0 \\
\hline \multicolumn{6}{|l|}{ Sud Chemie } \\
\hline DESICCANT & 0095 & General Commodity & $x$ & $x$ & $x$ \\
\hline DESICCANT & 0095 & SLDT & $x$ & $x$ & $x$ \\
\hline DESICCANT & 0095 & SLDV & $x$ & $x$ & $x$ \\
\hline DESICCANT & 0095 & SLDP & $x$ & $x$ & $x$ \\
\hline DESICCANT & 0095 & CVM/SLDC & $\mathrm{x}$ & $\mathrm{x}$ & $\mathrm{x}$ \\
\hline
\end{tabular}




\begin{tabular}{|c|c|c|c|c|c|}
\hline Nomenclature & Part Number & End Item & $\mathrm{SH}$ & $\mathrm{R}$ & $\mathrm{O}$ \\
\hline DESICCANT & 0095 & MDS & $x$ & $x$ & $x$ \\
\hline \multicolumn{6}{|l|}{ SuperLogics } \\
\hline KIT & & SafePass CAS & M & $x$ & $x$ \\
\hline \multicolumn{6}{|l|}{ TDK Lambda } \\
\hline POWER SUPPLY, 48 VDC & DPP480-48-1 & Serco Wireless Network & $\mathrm{x}$ & $\mathrm{x}$ & $x$ \\
\hline \multicolumn{6}{|l|}{ ThermoFisher } \\
\hline ADAPTER & GOVC000493 & ThermoFisher & $x$ & $\mathrm{x}$ & $x$ \\
\hline ADAPTER, PROTECTIVE & MEVE339 & ThermoFisher & $\mathrm{x}$ & $\mathrm{x}$ & $\mathrm{x}$ \\
\hline AIR CONDITIONER, XMHC & VEIN93 & ThermoFisher & M & $x$ & $x$ \\
\hline ASSEMBLY, CABLE HARNESS, EXT TX & YP11702228A & ThermoFisher & $x$ & $x$ & $x$ \\
\hline ASSEMBLY, CABLE HARNESS, EXT TX & YP11702228B & ThermoFisher & $\mathrm{x}$ & $\mathrm{x}$ & $\mathrm{x}$ \\
\hline ASSEMBLY, CABLE HARNESS, EXT TX & YP11702230A & ThermoFisher & $x$ & $x$ & $x$ \\
\hline ASSEMBLY, CABLE HARNESS, EXT TX & YP11702230B & ThermoFisher & $x$ & $x$ & $x$ \\
\hline ASSEMBLY, CABLE, 12 VDC FAN & YP11702479 & ThermoFisher & $\mathrm{x}$ & $\mathrm{x}$ & $\mathrm{x}$ \\
\hline ASSEMBLY, JUNCTION BOX & GOVA000102 & ThermoFisher & M & $x$ & $x$ \\
\hline ASSEMBLY, LH, RX(B) SENSOR & YP11702251-1 & ThermoFisher & $x$ & $x$ & $x$ \\
\hline ASSEMBLY, LH, TX(A) SENSOR & YP11702251 & ThermoFisher & $x$ & $x$ & $x$ \\
\hline ASSEMBLY, NAI, COMPLETE & YP11702006 & ThermoFisher & M & $x$ & $x$ \\
\hline ASSEMBLY, PCB, LED CIRCUIT & $5687 C$ & ThermoFisher & $x$ & $x$ & $x$ \\
\hline ASSEMBLY, RH, RX(A) SENSOR & YP11702250-1 & ThermoFisher & $x$ & $x$ & $x$ \\
\hline ASSEMBLY, RH, TX(B) SENSOR & YP11702250 & ThermoFisher & $x$ & $x$ & $x$ \\
\hline ASSEMBLY, SURGE ARRESTOR, 40A $120 \mathrm{~V}$ & MEVE367 & ThermoFisher & $x$ & $x$ & $x$ \\
\hline BOARD, CONTROLLER, BATTERY & $5681 \mathrm{~A}$ & ThermoFisher & $x$ & $x$ & $x$ \\
\hline BOARD, COVERSION, 24V DC DIGITAL IO & CONV1 & ThermoFisher & $x$ & $x$ & $x$ \\
\hline BOARD, DISPLAY & GOVC000167 & ThermoFisher & $x$ & $x$ & $x$ \\
\hline BOARD, MAIN CONTROL & GOVC000172 & ThermoFisher & M & $x$ & $x$ \\
\hline CABLE, CL, BFR/SPL TO SURGE PROTECTOR & YP11702499 & ThermoFisher & $\mathrm{x}$ & $\mathrm{x}$ & $\mathrm{x}$ \\
\hline CABLE, DC POWER, BOTTOM FAN & YP11702488 & ThermoFisher & $x$ & $x$ & $\mathrm{x}$ \\
\hline
\end{tabular}




\begin{tabular}{|c|c|c|c|c|c|}
\hline Nomenclature & Part Number & End Item & $\mathrm{SH}$ & $\mathrm{R}$ & $\mathrm{O}$ \\
\hline CABLE, DC POWER, TOP FAN & YP11702487 & ThermoFisher & $x$ & $x$ & $x$ \\
\hline CABLE, FIBER, 30 FT SC & YP11702523 & ThermoFisher & $x$ & $x$ & $x$ \\
\hline CABLE, FIBER, CONV TO COUPLER & YP11702500 & ThermoFisher & $x$ & $x$ & $x$ \\
\hline CABLE, LAN, POE SW RS485 LAN CON & YP11702497 & ThermoFisher & $x$ & $x$ & $x$ \\
\hline CABLE, LAN, POE SW TO TEMP BS & YP11702496 & ThermoFisher & $x$ & $x$ & $x$ \\
\hline CABLE, POWER, 48 VDC TO MON PCB & YP11702492 & ThermoFisher & $x$ & $x$ & $x$ \\
\hline CABLE, POWER, IN TO TB & YP11702489 & ThermoFisher & $x$ & $x$ & $x$ \\
\hline CABLE, POWER, MON PCB TO 48 VDC TB & YP11702494 & ThermoFisher & $x$ & $x$ & $x$ \\
\hline CABLE, POWER, TB TO 48 VDC PS & YP11702490 & ThermoFisher & $x$ & $x$ & $x$ \\
\hline CHARGER, BATTERY, 24V DC & GOVC000166 & ThermoFisher & M & $x$ & $x$ \\
\hline CIRCUIT BREAKER, 1P 120 VAC, 10A & FUCB24 & ThermoFisher & $x$ & $x$ & $x$ \\
\hline CIRCUIT BREAKER, 1P 120 VAC, 15A & FUCB25 & ThermoFisher & $x$ & $x$ & $x$ \\
\hline CIRCUIT BREAKER, 1P 120 VAC, 20A & FUCB26 & ThermoFisher & $x$ & $x$ & $x$ \\
\hline CIRCUIT BREAKER, 1P $120 \mathrm{VAC}, 2 \mathrm{~A}$ & FUCB22 & ThermoFisher & $x$ & $x$ & $x$ \\
\hline CIRCUIT BREAKER, 1P $120 \mathrm{VAC}, 5 \mathrm{~A}$ & FUCB23 & ThermoFisher & $x$ & $x$ & $x$ \\
\hline COMPACT FLASH, 8 GB & GoVC000119 & ThermoFisher & $x$ & $x$ & $x$ \\
\hline COUPLER, SC DUPLEX, MALE TO MALE & COHD282 & ThermoFisher & $x$ & $x$ & $x$ \\
\hline CRATE & & ThermoFisher & $x$ & $x$ & $x$ \\
\hline FAN, CIRCULATION, 115 VAC 4 INCH AXIAL & FNAC10 & ThermoFisher & $x$ & $x$ & $x$ \\
\hline FILTER, SINGLE PHASE, EMI/RFI & LFTR001 & ThermoFisher & $x$ & $x$ & $x$ \\
\hline FUSE, 10A & GOVC000295 & ThermoFisher & $x$ & $x$ & $x$ \\
\hline FUSE, 8A & GOVC000296 & ThermoFisher & $x$ & $x$ & $x$ \\
\hline HARNESS, WIRE, COMM POWER & ER6087C & ThermoFisher & $x$ & $x$ & $x$ \\
\hline HARNESS, WIRE, DC POWER & YP11702481 & ThermoFisher & $x$ & $x$ & $x$ \\
\hline HARNESS, WIRE, NAI DET EXT 1 & YP11702483 & ThermoFisher & $x$ & $x$ & $x$ \\
\hline HARNESS, WIRE, NAI DET EXT 2 & YP11702484 & ThermoFisher & $x$ & $x$ & $x$ \\
\hline HARNESS, WIRE, NAI DET EXT 3 & YP11702485 & ThermoFisher & $x$ & $x$ & $x$ \\
\hline HARNESS, WIRE, NAI DET EXT 4 & YP11702486 & ThermoFisher & $x$ & $x$ & $x$ \\
\hline HUB, TEMPERATURE, E4 & MEVE325 & ThermoFisher & $x$ & $x$ & $x$ \\
\hline
\end{tabular}




\begin{tabular}{|c|c|c|c|c|c|}
\hline Nomenclature & Part Number & End Item & $\mathrm{SH}$ & $\mathrm{R}$ & $\mathrm{O}$ \\
\hline IO UNIT, ETHERNET & MEVE361 & ThermoFisher & M & $x$ & $x$ \\
\hline KIT, HE3 & GOVA000219 & ThermoFisher & $x$ & $\mathrm{x}$ & $x$ \\
\hline KIT, PCS & GOVA000218 & ThermoFisher & $x$ & $x$ & $x$ \\
\hline KIT, RSP & GOVA000216 & ThermoFisher & $x$ & $x$ & $\mathrm{x}$ \\
\hline KIT, UPS & GOVA000217 & ThermoFisher & $\mathrm{H}$ & $x$ & $x$ \\
\hline KIT, VIS & GOVA000220 & ThermoFisher & $x$ & $x$ & $x$ \\
\hline LATCH, 3 POINT WITH KEY, ALTERED & ZP11702077 & ThermoFisher & $x$ & $x$ & $x$ \\
\hline MODULE, 4X4 & MEVE356 & ThermoFisher & $M$ & $x$ & $x$ \\
\hline MODULE, FIBER INSERT, SFF & MEVE355 & ThermoFisher & M & $x$ & $x$ \\
\hline MODULE, LANTRONIX & GOVC000171 & ThermoFisher & M & $x$ & $x$ \\
\hline MODULE, TIMING DISTRIBUTION & $5665 A$ & ThermoFisher & $x$ & $x$ & $x$ \\
\hline MOTOR, FAN & GOVC000168 & ThermoFisher & $x$ & $x$ & $x$ \\
\hline MOUNT, LOW PROFILE, VIBRATION & MMRU129 & ThermoFisher & $\mathrm{x}$ & $x$ & $\mathrm{x}$ \\
\hline MOUNT, PAN AND TILT & VEIN253 & ThermoFisher & $\mathrm{x}$ & $x$ & $x$ \\
\hline NETWORK CARD, X-CH, DUAL ETHERNET & $5648 \mathrm{D}$ & ThermoFisher & M & $x$ & $x$ \\
\hline PLUG, ISOLATION & MEVE317 & ThermoFisher & $x$ & $x$ & $x$ \\
\hline PLUG, PROTECTIVE, SURGE 120 VAC & MEVE316 & ThermoFisher & $x$ & $x$ & $x$ \\
\hline PLUG, PROTECTIVE, SURGE 24 VDC & GOVC000297 & ThermoFisher & $\mathrm{x}$ & $x$ & $x$ \\
\hline PLUG, PROTECTIVE, SURGE 5 VDC & MEVE358 & ThermoFisher & $x$ & $x$ & $x$ \\
\hline POWER SUPPLY, 10A 24 VDC & GOVC000299 & ThermoFisher & $x$ & $x$ & $x$ \\
\hline POWER SUPPLY, 2A 12 VDC & MEVE360 & ThermoFisher & $x$ & $x$ & $x$ \\
\hline POWER SUPPLY, AC DC, 12V & MEVE336 & ThermoFisher & $\mathrm{x}$ & $x$ & $x$ \\
\hline POWER SUPPLY, AC DC, 48V & MEVE337 & ThermoFisher & $\mathrm{x}$ & $x$ & $x$ \\
\hline REMOTE IO UNIT, ETHERNET, REMOTE TRACK & MEVE362 & ThermoFisher & $x$ & $x$ & $x$ \\
\hline SENSOR, TEMPERATURE & MEVE326 & ThermoFisher & $x$ & $x$ & $x$ \\
\hline SENSOR, TEMPERATURE, CABINET & $5659 B$ & ThermoFisher & $x$ & $x$ & $x$ \\
\hline SLIDE, ALTERED ITEM & HDMI284 & ThermoFisher & $x$ & $x$ & $x$ \\
\hline STRAP, GROUNDING & YP11688037 & ThermoFisher & $x$ & $x$ & $x$ \\
\hline SUN SHIELD & VEIN255 & ThermoFisher & $x$ & $x$ & $x$ \\
\hline
\end{tabular}




\begin{tabular}{|c|c|c|c|c|c|}
\hline Nomenclature & Part Number & End Item & $\mathrm{SH}$ & $\mathrm{R}$ & $\mathrm{O}$ \\
\hline SWITCH, ETHERNET, UNMANAGED & MEVE368 & ThermoFisher & $\mathrm{x}$ & $x$ & $x$ \\
\hline SWITCH, SERIAL TO ETHERNET & MEVE369 & ThermoFisher & $x$ & $x$ & $x$ \\
\hline TRIM, BLACK BULB, 7/16 O.D. & HDTA201 & ThermoFisher & $x$ & $x$ & $x$ \\
\hline \multicolumn{6}{|l|}{ Titan Wireless } \\
\hline INJECTOR, POWER OVER ETHERNET & 0466B & RAVEN CAS & $x$ & $x$ & $x$ \\
\hline \multicolumn{6}{|l|}{ Tripp Lite } \\
\hline CABLE & & SafePass CAS & $\mathrm{x}$ & $x$ & $x$ \\
\hline CABLE, EXTENDER, KVM & & SafePass CAS & $x$ & $\mathrm{x}$ & $\mathrm{x}$ \\
\hline FAN, RACK MOUNT, FAN ROOF & $0459 \mathrm{~F}$ & RAVEN CAS & $\mathrm{x}$ & $x$ & $x$ \\
\hline KIT, CABLE, B020-008-17 CONSOLE & & SafePass CAS & $x$ & $\mathrm{x}$ & $x$ \\
\hline KIT, CABLE, KVM & 0459K1 & RAVEN CAS & $x$ & $x$ & $x$ \\
\hline PATCH PANEL, 16 PORT & & SafePass CAS & $x$ & $x$ & $x$ \\
\hline PATCH PANEL, 24 PORT & & SafePass CAS & $\mathrm{x}$ & $\mathrm{x}$ & $\mathrm{x}$ \\
\hline RACK, $25 \mathrm{U}$ & & SafePass CAS & M & $x$ & $x$ \\
\hline RACK, ENCLOSED, 19 INCH & 0459R & RAVEN CAS & M & $x$ & $x$ \\
\hline SHELF, RACK MOUNT & 04595 & RAVEN CAS & $x$ & $\mathrm{x}$ & $x$ \\
\hline SHELF, RACK MOUNT, SLIDING & 0459SS & RAVEN CAS & $x$ & $x$ & $x$ \\
\hline SWITCH, 8 PORT KVM & & SafePass CAS & M & $\mathrm{x}$ & $\mathrm{x}$ \\
\hline SWITCH, KVM & 0459K & RAVEN CAS & $x$ & $x$ & $x$ \\
\hline UPS, 1500 VA, RACK OR TOWER & 0476A & RAVEN CAS & $\mathrm{H}$ & $x$ & $x$ \\
\hline UPS, 1500VA & & SafePass CAS & $\mathrm{H}$ & $\mathrm{x}$ & $x$ \\
\hline UPS, 750 VA & 0476 & RAVEN CAS & $\mathrm{H}$ & $x$ & $x$ \\
\hline UPS, 750VA & & SafePass CAS & $\mathrm{H}$ & $x$ & $x$ \\
\hline \multicolumn{6}{|l|}{ TSA } \\
\hline ASSEMBLY, MEDIA CONVERTER & 8590 & CVM/SLDC & $x$ & $x$ & $x$ \\
\hline ASSEMBLY, MEDIA CONVERTER & 8590 & SLDV & $x$ & $x$ & $x$ \\
\hline ASSEMBLY, MEDIA CONVERTER & 8590 & SLDP & $x$ & $\mathrm{x}$ & $x$ \\
\hline ASSEMBLY, MEDIA CONVERTER & 8590 & SLDT & $\mathrm{x}$ & $\mathrm{x}$ & $\mathrm{x}$ \\
\hline
\end{tabular}




\begin{tabular}{|c|c|c|c|c|c|}
\hline Nomenclature & Part Number & End Item & $\mathrm{SH}$ & $\mathrm{R}$ & 0 \\
\hline ASSEMBLY, MEDIA CONVERTER & 8590 & MDS & $x$ & $x$ & $x$ \\
\hline BATTERY, 18AH $12 \mathrm{~V}$ & $6456 \mathrm{~A}$ & SLDT & H & $x$ & $x$ \\
\hline BATTERY, 18AH $12 \mathrm{~V}$ & $6456 \mathrm{~A}$ & SLDV & $\mathrm{H}$ & $x$ & $x$ \\
\hline BATTERY, 18AH $12 \mathrm{~V}$ & $6456 \mathrm{~A}$ & SLDP & H & $x$ & $x$ \\
\hline BATTERY, 26AH $12 \mathrm{~V}$ & $6456 \mathrm{~B}$ & SLDT & $\mathrm{H}$ & $x$ & $x$ \\
\hline BATTERY, 26AH $12 \mathrm{~V}$ & $6456 B$ & SLDP & H & $x$ & $x$ \\
\hline BATTERY, 26AH $12 \mathrm{~V}$ & $6456 \mathrm{~B}$ & SLDV & $\mathrm{H}$ & $x$ & $x$ \\
\hline BOARD, 8403 & 8403AP & SLDP & $x$ & $x$ & $x$ \\
\hline BOARD, 8403 & 8403AP & CVM/SLDC & $x$ & $x$ & $x$ \\
\hline BOARD, 8403 & 8403AP & SLDT & $x$ & $x$ & $x$ \\
\hline BOARD, 8403 & 8403AP & SLDV & $x$ & $x$ & $x$ \\
\hline BOARD, 8453 & $8453 G$ & MDS & $x$ & $x$ & $x$ \\
\hline BOARD, 8453 & $8453 G$ & SLDT & $x$ & $x$ & $x$ \\
\hline BOARD, 8453 & $8453 G$ & SLDP & $x$ & $x$ & $x$ \\
\hline BOARD, 8453 & $8453 \mathrm{G}$ & SLDV & $x$ & $x$ & $x$ \\
\hline BOARD, 8453 & $8453 G$ & CVM/SLDC & $x$ & $x$ & $x$ \\
\hline BOARD, SC-771 & $8751 \mathrm{AA}$ & SLDT & $x$ & $x$ & $x$ \\
\hline BOARD, SC-771 & 8751AA & MDS & $x$ & $x$ & $x$ \\
\hline BOARD, SC-771 & $8751 \mathrm{AA}$ & SLDV & $x$ & $x$ & $x$ \\
\hline BOARD, SC-771 & $8751 \mathrm{AA}$ & CVM/SLDC & $x$ & $x$ & $x$ \\
\hline BOARD, SC-771 & $8751 \mathrm{AA}$ & SLDP & $x$ & $x$ & $x$ \\
\hline BOARD, SCA-774 & 8754 & MDS & $x$ & $x$ & $x$ \\
\hline BOARD, SCA-774 & 8754 & SLDT & $x$ & $x$ & $x$ \\
\hline BOARD, SCA-774 & 8754 & CVM/SLDC & $x$ & $x$ & $x$ \\
\hline BOARD, SCA-774 & 8754 & SLDP & $x$ & $x$ & $x$ \\
\hline BOARD, SCA-774 & 8754 & SLDV & $x$ & $x$ & $x$ \\
\hline BRACKET & 3450 & SLDV & $x$ & $x$ & $x$ \\
\hline CABLE & $0301 C$ & SLDV & $x$ & $x$ & $x$ \\
\hline CABLE & $0301 \mathrm{E}$ & SLDV & $x$ & $x$ & $x$ \\
\hline
\end{tabular}




\begin{tabular}{|c|c|c|c|c|c|}
\hline Nomenclature & Part Number & End Item & $\mathrm{SH}$ & $\mathrm{R}$ & $\mathrm{O}$ \\
\hline CABLE & 0344D & SLDV & $\mathrm{x}$ & $\mathrm{x}$ & $\mathrm{x}$ \\
\hline CABLE & 0360 & SLDV & $x$ & $x$ & $x$ \\
\hline CABLE & $0360 \mathrm{~A}$ & SLDV & $x$ & $x$ & $x$ \\
\hline CABLE & 0360B & SLDV & $x$ & $x$ & $x$ \\
\hline CABLE & $2802 B$ & SLDV & $x$ & $x$ & $x$ \\
\hline CABLE, 2 CONDUCTOR 18AWG, $100 \mathrm{FT}$ & & RAVEN CAS & $\mathrm{x}$ & $\mathrm{x}$ & $x$ \\
\hline CABLE, COAX & 0349 & CVM/SLDC & $x$ & $x$ & $x$ \\
\hline CABLE, COAX & 0349 & CVM/SLDC & $x$ & $x$ & $x$ \\
\hline CABLE, COAX & 0350 & SLDV & $x$ & $x$ & $x$ \\
\hline CABLE, COAX & 0352 & SLDP & $x$ & $x$ & $x$ \\
\hline CABLE, COAX & 0353 & SLDT & $x$ & $x$ & $x$ \\
\hline CAMERA, NETWORK & $0460 C$ & RAVEN CAS & M & $x$ & $x$ \\
\hline CHARGER, 4A 15V & $6463 B$ & CVM/SLDC & $x$ & $x$ & $x$ \\
\hline CHARGER, 4A 15V & $6463 B$ & SLDP & $x$ & $x$ & $x$ \\
\hline CHARGER, 5A 15V & $6463 C$ & SLDT & $x$ & $x$ & $x$ \\
\hline CHARGER, 5A 15V & $6463 C$ & SLDV & $x$ & $x$ & $x$ \\
\hline CIRCUIT BREAKER & $7802 B$ & RAVEN CAS & $x$ & $x$ & $x$ \\
\hline COMPACT FLASH, PEN DRIVE, WITH CABLE & 0463B & RAVEN CAS & $x$ & $x$ & $x$ \\
\hline CONNECTOR & $2725 \mathrm{~A}$ & SLDV & $x$ & $x$ & $x$ \\
\hline CONNECTOR & $2725 \mathrm{~A}$ & SLDT & $x$ & $x$ & $x$ \\
\hline CONNECTOR, MHV, FEMALE TO FEMALE & 2515 & SLDT & $x$ & $x$ & $x$ \\
\hline CONTROL PLATE, MASTER & 8774 & SLDV & $x$ & $x$ & $x$ \\
\hline CONTROL PLATE, SLAVE & $8774 \mathrm{~A}$ & SLDV & $x$ & $x$ & $x$ \\
\hline CONTROLLER, SC-770 & 9411 & SLDT & $M$ & $x$ & $x$ \\
\hline CONTROLLER, SC-770 & 9411 & MDS & M & $x$ & $x$ \\
\hline CONTROLLER, SC-770 & 9411 & SLDV & $M$ & $x$ & $x$ \\
\hline CONTROLLER, SC-770 & 9411 & CVM/SLDC & M & $x$ & $x$ \\
\hline CONTROLLER, SC-770 & 9411 & SLDP & $M$ & $x$ & $x$ \\
\hline CONTROLLER, SCA-775, ANALOG & 9413 & MDS & M & $\mathrm{x}$ & $\mathrm{x}$ \\
\hline
\end{tabular}




\begin{tabular}{|c|c|c|c|c|c|}
\hline Nomenclature & Part Number & End Item & $\mathrm{SH}$ & $\mathrm{R}$ & $\mathrm{O}$ \\
\hline CONTROLLER, SCA-775, ANALOG & 9413 & SLDP & M & $x$ & $x$ \\
\hline CONTROLLER, SCA-775, ANALOG & 9413 & CVM/SLDC & M & $x$ & $x$ \\
\hline CONTROLLER, SCA-775, ANALOG & 9413 & SLDV & M & $x$ & $x$ \\
\hline CONTROLLER, SCA-775, ANALOG & 9413 & SLDT & M & $x$ & $x$ \\
\hline DETECTOR, GAMMA & $8200 A$ & SLDV & M & $x$ & $x$ \\
\hline DETECTOR, GAMMA & $8200 \mathrm{~A}$ & SLDP & M & $x$ & $x$ \\
\hline DETECTOR, GAMMA & $8200 \mathrm{~A}$ & CVM/SLDC & M & $x$ & $x$ \\
\hline DETECTOR, GAMMA, 12 INCH BY $48 \mathrm{INCH}$ & 8244 & SLDT & M & $x$ & $x$ \\
\hline DRIVER, SIREN & $7346 B$ & SLDT & $x$ & $x$ & $x$ \\
\hline DRIVER, SIREN & $7346 \mathrm{~B}$ & SLDV & $x$ & $x$ & $x$ \\
\hline ENCLOSURE & 6985 & SLDV & $x$ & $x$ & $x$ \\
\hline ENCLOSURE & $6985 A$ & SLDV & $x$ & $x$ & $x$ \\
\hline ENCLOSURE & 6987 & SLDV & $x$ & $x$ & $x$ \\
\hline ENCLOSURE & $6987 \mathrm{~A}$ & SLDV & $x$ & $x$ & $x$ \\
\hline FILTER, AC LINE & 0418 & SLDT & $x$ & $x$ & $x$ \\
\hline FILTER, AC LINE & 0418 & MDS & $x$ & $x$ & $x$ \\
\hline FILTER, AC LINE & 0418 & SLDP & $x$ & $x$ & $x$ \\
\hline FILTER, AC LINE & 0418 & SLDV & $x$ & $x$ & $x$ \\
\hline FILTER, AC LINE & 0418 & CVM/SLDC & $x$ & $x$ & $x$ \\
\hline FUSE, 5 AMP & $7170 B$ & MDS & $x$ & $x$ & $x$ \\
\hline FUSE, 5 AMP & $7170 \mathrm{~B}$ & SLDT & $x$ & $x$ & $x$ \\
\hline FUSE, 5 AMP & $7170 \mathrm{~B}$ & SLDP & $x$ & $x$ & $x$ \\
\hline FUSE, 5 AMP & $7170 \mathrm{~B}$ & SLDV & $x$ & $x$ & $x$ \\
\hline FUSE, 5 AMP & $7170 \mathrm{~B}$ & CVM/SLDC & $x$ & $x$ & $x$ \\
\hline HE3 TUBE & $6720 C$ & SLDV & $x$ & $x$ & $x$ \\
\hline HEATER STRIP & 7230 & SLDV & $x$ & $x$ & $x$ \\
\hline KIT, SPEED SENSOR & 6661 & SLDV & $M$ & $x$ & $x$ \\
\hline KIT, SPEED SENSOR & 6661 & SLDT & M & $x$ & $x$ \\
\hline LEAD SHIELDS & 4804 & SLDV & $x$ & $x$ & $x$ \\
\hline
\end{tabular}




\begin{tabular}{|c|c|c|c|c|c|}
\hline Nomenclature & Part Number & End Item & $\mathrm{SH}$ & $\mathrm{R}$ & $\mathrm{O}$ \\
\hline LEAD TROUGH & $4804 \times 1$ & SLDV & $\mathrm{x}$ & $\mathrm{x}$ & $\mathrm{x}$ \\
\hline LIGHT, FAULT, AMBER & 7354 & SLDT & $x$ & $x$ & $x$ \\
\hline LIGHT, FAULT, AMBER & 7354 & SLDP & $x$ & $x$ & $x$ \\
\hline LIGHT, FAULT, AMBER & 7354 & SLDV & $x$ & $x$ & $x$ \\
\hline LIGHT, STROBE, BLUE & $7346 \mathrm{~A}$ & SLDV & $x$ & $x$ & $x$ \\
\hline LIGHT, STROBE, BLUE & $7346 \mathrm{~A}$ & SLDT & $\mathrm{x}$ & $\mathrm{x}$ & $x$ \\
\hline LIGHT, STROBE, RED & 7346 & SLDT & $x$ & $x$ & $x$ \\
\hline LIGHT, STROBE, RED & 7346 & SLDV & $x$ & $x$ & $x$ \\
\hline MACH ALUM PLATE, MASTER & $3204 C$ & SLDV & $\mathrm{x}$ & $\mathrm{x}$ & $\mathrm{x}$ \\
\hline MACH ALUM PLATE, SLAVE & $3204 \mathrm{~B}$ & SLDV & $x$ & $x$ & $x$ \\
\hline MEDIA CONVERTER & 8590 & RAVEN CAS & $x$ & $x$ & $x$ \\
\hline MOUNT & $3450 \mathrm{D}$ & SLDV & $x$ & $x$ & $x$ \\
\hline PIPE CLAMPS & 3470 & SLDV & $x$ & $x$ & $x$ \\
\hline PMFX4, POTTED & $8699 P$ & CVM/SLDC & $x$ & $x$ & $x$ \\
\hline PMFX4, POTTED & $8699 P$ & SLDT & $x$ & $x$ & $x$ \\
\hline PMFX4, POTTED & $8699 P$ & SLDP & $x$ & $x$ & $x$ \\
\hline PMFX4, POTTED & $8699 \mathrm{P}$ & SLDV & $x$ & $x$ & $x$ \\
\hline POLY BLOCKS & 4875 & SLDV & $x$ & $x$ & $x$ \\
\hline POWER PLATE & $3204 \mathrm{E}$ & SLDV & $x$ & $x$ & $x$ \\
\hline POWER PLATE & $8774 B$ & SLDV & $x$ & $x$ & $x$ \\
\hline REFLECTOR, 3 INCH & $6669 R$ & CVM/SLDC & $x$ & $x$ & $x$ \\
\hline REMOTE CONTROL & $6692 R$ & SLDP & $x$ & $x$ & $x$ \\
\hline SENSOR, OCCUPANCY & $6692 \mathrm{~W}$ & SLDP & $x$ & $x$ & $x$ \\
\hline SENSOR, PHOTO RELEECTOR & $6669 \mathrm{~A}$ & CVM/SLDC & $x$ & $x$ & $x$ \\
\hline SENSOR, RADAR & 6661TEU & SLDT & M & $x$ & $x$ \\
\hline SENSOR, ULTRASONIC & $6661 B$ & SLDT & $x$ & $x$ & $x$ \\
\hline SENSOR, ULTRASONIC & $6666 \mathrm{D} 3$ & SLDV & $x$ & $x$ & $x$ \\
\hline SIREN & $7346 C$ & SLDV & $x$ & $x$ & $x$ \\
\hline SIREN & $7346 C$ & SLDT & $x$ & $\mathrm{x}$ & $\mathrm{x}$ \\
\hline
\end{tabular}




\begin{tabular}{|c|c|c|c|c|c|}
\hline Nomenclature & Part Number & End Item & $\mathrm{SH}$ & $\mathrm{R}$ & 0 \\
\hline SOFTWARE, PC, RAVEN & & RAVEN CAS & M & $x$ & $x$ \\
\hline SOFTWARE, RAVEN, CONCENTRATOR & & RAVEN CAS & $x$ & $x$ & $x$ \\
\hline SONALERT & 6440 & CVM/SLDC & $x$ & $x$ & $x$ \\
\hline SONALERT & 6440 & SLDP & $x$ & $x$ & $x$ \\
\hline SWITCH, TAMPER, MAGNETIC & 7818 & SLDV & $x$ & $x$ & $x$ \\
\hline SWITCH, TAMPER, MAGNETIC & 7818 & SLDT & $x$ & $\mathrm{x}$ & $\mathrm{x}$ \\
\hline SWITCH, TAMPER, MAGNETIC & 7818 & SLDP & $\mathrm{x}$ & $\mathrm{x}$ & $\mathrm{x}$ \\
\hline TERMINAL & 2532 & SLDV & $\mathrm{x}$ & $x$ & $\mathrm{x}$ \\
\hline TERMINAL & $2533 A$ & SLDV & $x$ & $x$ & $x$ \\
\hline TERMINAL & $2533 B$ & SLDV & $\mathrm{x}$ & $x$ & $x$ \\
\hline TERMINAL & $2533 C$ & SLDV & $x$ & $x$ & $x$ \\
\hline TERMINAL & $2533 C$ & SLDV & $x$ & $x$ & $x$ \\
\hline TERMINAL & $2533 D$ & SLDV & $x$ & $x$ & $x$ \\
\hline TERMINAL & $2533 F$ & SLDV & $x$ & $\mathrm{x}$ & $\mathrm{x}$ \\
\hline TERMINAL STRIP & 2533E & SLDV & $\mathrm{x}$ & $\mathrm{x}$ & $\mathrm{x}$ \\
\hline TERMINAL STRIP & 2533E & RAVEN CAS & $\mathrm{x}$ & $x$ & $\mathrm{x}$ \\
\hline TIES, CABLE & $3450 \mathrm{C}$ & SLDV & $x$ & $\mathrm{x}$ & $\mathrm{x}$ \\
\hline UPS, 1500VA & 0476A & RAVEN CAS & $\mathrm{H}$ & $x$ & $x$ \\
\hline VD580, POTTED & 8870AP & CVM/SLDC & $x$ & $\mathrm{x}$ & $x$ \\
\hline VD580, POTTED & 8870AP & SLDV & $x$ & $\mathrm{x}$ & $x$ \\
\hline VD580, POTTED & 8870AP & SLDT & $\mathrm{x}$ & $x$ & $x$ \\
\hline VD580, POTTED & 8870AP & SLDP & $\mathrm{x}$ & $\mathrm{x}$ & $\mathrm{x}$ \\
\hline VD580, POTTED & 8870AP & MDS & $\mathrm{x}$ & $\mathrm{x}$ & $x$ \\
\hline \multicolumn{6}{|l|}{ TSi Power } \\
\hline VOLTAGE REGULATOR, OUTDOOR, 172 TO 276 VAC INPUT & VRx-2100-8071 & Schneider CAS & $x$ & $x$ & $x$ \\
\hline \multicolumn{6}{|l|}{ Wago } \\
\hline CONTROLLER, IO ETHERNET & $750-841$ & Siemens OCR & $x$ & $x$ & $\mathrm{x}$ \\
\hline DIGITAL INPUT, IO ETHERNET & $750-432$ & Siemens OCR & $x$ & $x$ & $\mathrm{x}$ \\
\hline
\end{tabular}




\begin{tabular}{|c|c|c|c|c|c|}
\hline Nomenclature & Part Number & End Item & $\mathrm{SH}$ & $\mathrm{R}$ & $\mathrm{O}$ \\
\hline DIGITAL INPUT, IO ETHERNET & $750-504$ & Siemens OCR & $x$ & $x$ & $x$ \\
\hline END MODULE, IO ETHERNET & $750-600$ & Siemens OCR & $x$ & $x$ & $\mathrm{X}$ \\
\hline POWER SUPPLY, IO ETHERNET, IO & $750-612$ & Siemens OCR & $x$ & $x$ & $\mathrm{x}$ \\
\hline POWER SUPPLY, IO ETHERNET, IO & $787-612$ & Siemens OCR & $x$ & $x$ & $x$ \\
\hline SUPPLY MODULE, IO ETHERNET & $750-610$ & Siemens OCR & $x$ & $\mathrm{X}$ & $\mathrm{X}$ \\
\hline \multicolumn{6}{|l|}{ Weidmuller } \\
\hline BATTERY, BACKUP UNIT & 9916280012 & Utility Panels & $\mathrm{H}$ & $x$ & $\mathrm{O}$ \\
\hline POWER SUPPLY, 12 VDC, 160W & 9925340012 & Utility Panels & $x$ & $\mathrm{x}$ & $\mathrm{O}$ \\
\hline \multicolumn{6}{|l|}{ Zerosurge } \\
\hline SURGE SUPPRESSOR & TVS230LC10 & Utility Panels & $x$ & $x$ & $\mathrm{O}$ \\
\hline
\end{tabular}




\section{Master Parts Catalog by End Items}

\begin{tabular}{|c|c|c|c|}
\hline Manufacturer & Nomenclature & Part Number & Description \\
\hline APS OCR & OCR & & Identification \\
\hline APS Technology & BOARD, SINGLE, IO TO ETHERNET & 24-016-015 & IO To Ethernet- Single Board Computer \\
\hline APS Technology & CAMERA, TOP LENS MOUNTED, 3.0MP & 29002-001 & Camera- 3.0 MP Top Camera with Lens Mounted in Exterior Enclosure \\
\hline APS Technology & CAMERA, TOP LENS MOUNTED, 3.0MP & 29-0019-001 & Camera- 2.0 MP Back Camera with Lens Mounted in Exterior Enclosure \\
\hline APS Technology & DETECTOR, LOOP & $24-0016-014$ & Loop detector \\
\hline APS Technology & DETECTOR, LOOP, EXTENDED PRESENCE & $42-0002-001$ & Loop Detector- w/ extended presence \\
\hline APS Technology & DETECTOR, RADAR, FRONT AND REAR SPEED & 80-0001-001 & Radar- Front/Rear Speed Detector \\
\hline APS Technology & ILLUMINATOR, SIDE FLAT ARRAY & $66-0003002$ & Illuminator-Side Flat Array Illuminator Mounted in Exterior Enclosure \\
\hline APS Technology & ILLUMINATOR, SIDE FLAT ARRAY & $66-0003001$ & Illuminator- Back Collared Illuminator Mounted in Exterior Enclosure \\
\hline APS Technology & $\begin{array}{l}\text { MEDIA CONVERTER, TRANSCEIVER MODULE, } \\
\text { MULTIMODE }\end{array}$ & & Media Converter- Transceiver module 100Base, Multimode LC \\
\hline APS Technology & POWER SUPPLY, 12 VDC & 24-0016-029 & Power Supply- 12vDC, 50w, Din Rail Mount, NEC Class 2 \\
\hline APS Technology & POWER SUPPLY, 24 VDC & 24-0016-030 & Power supply $24 \mathrm{vDC}$ \\
\hline APS Technology & RECEPTACLE, REMOTELY MANAGED & 24-0016-018 & Remotely managed receptacle \\
\hline APS Technology & SENSOR, BEAM RECEIVER AND TRANSMITTER & 44-0001-001 & Sensor- Beam Receiver/Transmitter \\
\hline APS Technology & SERVER, OCS OCR & 00-0001-003 & OCS/OCR Server \\
\hline APS Technology & SWITCH, 24 PORT, GIGABIT & $56-0015-002$ & Switch- 24-port 10/100 + 4-port Gigabit Switch with WebView and PoE \\
\hline CVM/SLDC & Fixed & & Detection \\
\hline Sud Chemie & DESICCANT & 0095 & Desiccant \#6 \\
\hline TSA & ASSEMBLY, MEDIA CONVERTER & 8590 & Media Converter Assembly \\
\hline TSA & BOARD, 8403 & 8403AP & HHV-448D BOARD \\
\hline TSA & BOARD, 8453 & $8453 G$ & LD-260B \\
\hline TSA & BOARD, SC-771 & 8751AA & SC-771 R7 BOARD \\
\hline TSA & BOARD, SCA-774 & 8754 & SCA-774 BOARD \\
\hline TSA & CABLE, COAX & 0349 & CVM COAX LOT \\
\hline TSA & CABLE, COAX & 0349 & CVM COAX LOT \\
\hline TSA & CHARGER, 4A 15V & $6463 B$ & CHARGER 15 V 4 AMP \\
\hline
\end{tabular}




\begin{tabular}{|c|c|c|c|}
\hline Manufacturer & Nomenclature & Part Number & Description \\
\hline TSA & CONTROLLER, SC-770 & 9411 & SC-770 Controller \\
\hline TSA & CONTROLLER, SCA-775, ANALOG & 9413 & SCA-775 Controller (Analog) \\
\hline TSA & DETECTOR, GAMMA & $8200 \mathrm{~A}$ & DA630 (GAMMA DETECTOR) \\
\hline TSA & FILTER, AC LINE & 0418 & AC Line Filter \\
\hline TSA & FUSE, 5 AMP & $7170 \mathrm{~B}$ & 5 AMP FUSE (LD-260) \\
\hline TSA & PMFX4, POTTED & $8699 P$ & PMFX4 BOX ASSEMBLY (POTTED) \\
\hline TSA & REFLECTOR, 3 INCH & $6669 \mathrm{R}$ & 3" REFLECTOR (CVM) \\
\hline TSA & SENSOR, PHOTO RELEECTOR & $6669 \mathrm{~A}$ & PHOTO REFLECTOR SENSOR (CVM) \\
\hline TSA & SONALERT & 6440 & SONALERT \\
\hline TSA & VD580, POTTED & 8870AP & GAMMA DETECTOR CAP VD-580 (POTTED) \\
\hline General Commodity & \multicolumn{2}{|c|}{ System Support and Physical Protection } & Infrastructure \\
\hline Sud Chemie & DESICCANT & 0095 & Desiccant \#6 \\
\hline HTS OCR & OCR & & Identification \\
\hline HTS & CABLE, SMB, MALE & 550054 & Cable SMB-F male \\
\hline HTS & CONNECTOR, BCN, MALE TO DOUBLE & 509325 & Connector BCN M to Double \\
\hline HTS & DETECTOR, LOOP, MATRIX & 545010 & Loop Detector Matrix \\
\hline HTS & DETECTOR, LOOP, SOCKET MATRIX & 545011 & Loop Detector Socket Matrix \\
\hline HTS & FRAME GRABBER & 560217 & Frame Grabber Santos \\
\hline HTS & IO CARD & 550117 & I/O Card \\
\hline HTS & LICENSE, USB PLUG & 580044 & License Plug USB \\
\hline HTS & RECEIVER & 547008 & Receiver \\
\hline HTS & SERVER, PENTIUM 4 & 940000 & Computer-PENTIUM-4 \\
\hline HTS & SET, TERMINAL BOARD & 580136 & TBL-6822 Set \\
\hline HTS & SOFTWARE, RELEASE CD & 550045 & Software Release CD \\
\hline HTS & SYNCHRONIZATION GENERATOR & 910373 & Sync. Generator \\
\hline HTS & TRANSMITTER & 547007 & Transmitter \\
\hline HTS & VIDEO DISTRIBUTER & 910371 & Video Distributer \\
\hline Sony & CAMERA, REAR & 17COLREARO6ASSY & Rear Camera \\
\hline
\end{tabular}




\begin{tabular}{|c|c|c|c|c|}
\hline & Manufacturer & Nomenclature & Part Number & Description \\
\hline & Sony & CAMERA, SCH-2, YELLOW & 19BWOR3712500EL & Camera SCH-2 Yellow \\
\hline & Sony & CAMERA, SIDE & 17COLO00440ASSY & Side Camera \\
\hline & Sony & CAMERA, TOP & 17COLTOP044ASSY & Top Camera \\
\hline \multirow[t]{15}{*}{ ICIS OC } & & OCR & & Identification \\
\hline & Cisco & TRANCEIVER, FIBER CONVERTER, LXLH & GLC-LH-SM & CISCO Fiber Converters LXLH tranceiver \\
\hline & Generic Manufacturer & COUPLER, BNC, FEMALE TO FEMALE & & Coupler, BNC (standard F-F) \\
\hline & Generic Manufacturer & HDD, DISK ARRAY, 450GB & & Hard Drive, Disk Array, 450GB \\
\hline & Generic Manufacturer & HDD, REMOVABLE 15K, 146GB & 419814-001 & 146 GB 15kRPM U320 removable HDD \\
\hline & Generic Manufacturer & SPLITTER, BNC, FEMAL TO MALE TO FEMALE & & Splitter, BNC (standard T connector F-M-F) \\
\hline & Generic Manufacturer & WORK STATION, OCR, ACQUISITION AND WORKER & 430676-002 & Computer, OCR Acquisition/Worker \\
\hline & Hewlett Packard & SERVER, PROLIANT & DL380 G4 & HP ProLiant DL380 G4 \\
\hline & ICIS & CABLE, FC SC SM, 3M & FCSC-SMD-02 & Patch Cable - FC-SC-SM (3M) \\
\hline & ICIS & CABLE, LC SC SM, 2M & FCSC-SMD-02 & Patch Cable - LC-SC-SM (2M) \\
\hline & ICIS & CABLE, LC SC SM, 3M & 13-HPS108-3M & Patch Cable - LC-SC-SM (3M) \\
\hline & ICIS & CABLE, SC SC SM, 5M & SCSSTG16 & Patch Cable - SC-SC-SM (5M) \\
\hline & ICIS & RECEIVER, VIDEO, SM RX & $431489-002$ & Video Receiver, SM RX, 4V Out, 12V \\
\hline & ICIS & SERVER, ICIS APP & 431413-001 & Server, ICIS APP, DL380 G5 \\
\hline & ICIS & SWITCH TRANSCEIVER, GIGABIT EHTERNET, SFP & $421063-001$ & Switch Transceiver, SM, GB Ethernet, Industrial, SFP \\
\hline \multirow[t]{10}{*}{ MDS } & & Mobile & & Detection \\
\hline & Sud Chemie & DESICCANT & 0095 & Desiccant \#6 \\
\hline & TSA & ASSEMBLY, MEDIA CONVERTER & 8590 & Media Converter Assembly \\
\hline & TSA & BOARD, 8453 & $8453 G$ & LD-260B \\
\hline & TSA & BOARD, SC-771 & 8751AA & SC-771 R7 BOARD \\
\hline & TSA & BOARD, SCA-774 & 8754 & SCA-774 BOARD \\
\hline & TSA & CONTROLLER, SC-770 & 9411 & SC-770 Controller \\
\hline & TSA & CONTROLLER, SCA-775, ANALOG & 9413 & SCA-775 Controller (Analog) \\
\hline & TSA & FILTER, AC LINE & 0418 & AC Line Filter \\
\hline & TSA & FUSE, 5 AMP & $7170 \mathrm{~B}$ & 5 AMP FUSE (LD-260) \\
\hline
\end{tabular}




\begin{tabular}{|c|c|c|c|c|}
\hline & Manufacturer & Nomenclature & Part Number & Description \\
\hline & TSA & VD580, POTTED & 8870AP & GAMMA DETECTOR CAP VD-580 (POTTED) \\
\hline RAVEN & CAS & CAS & & Communications \\
\hline & ARC Wireless & MOUNT, RADIO, OUTDOOR & 0466M & Outdoor Radio Mounts \\
\hline & Bosch & ILLUMINATOR, IR & & IR Illuminator \\
\hline & CTL & MONITOR, FLAT PANEL, 19 INCH & 0455 & Monitor 19inch Flat Panel \\
\hline & Cybernet & WORK STATION, WITH COVER, WITH MOUSE & 0458A & Computer+Cover+Mouse \\
\hline & Deliberant & RADIO, OUTDOOR & 0466 & Outdoor Radio \\
\hline & Deliberant & RADIO, OUTDOOR & 0465B & Outdoor Radio \\
\hline & Deliberant & SURGE PROTECTOR & 0466A & Surge Protector \\
\hline & D-Link & ANTENNA, EXTERNAL & $0465 \mathrm{~A}$ & External Antenna \\
\hline & D-Link & WIRELESS ACCESS POINT & 0465 & Wireless Access Point \\
\hline & Enlight & SERVER, RACK MOUNT, $2 U$ & 0459A & 2U Rack Mount Server \\
\hline & Extreme CCTV & ILLUMINATOR, IR & $0460 \mathrm{~A}$ & IR Illuminator \\
\hline & GarrettCom & MOUNT, DIN RAIL & 0464BD & DIN-Rail Mount for ES42P \\
\hline & GarrettCom & POWER SUPPLY, SWITCH & $0464 C$ & Switch Power Supply \\
\hline & GarrettCom & SWITCH, 24 PORT & 0464E & Switch 24 Port \\
\hline & GarrettCom & SWITCH, 6 PORT, 12 VDC & 0464B & Switch 6 Port $12 \mathrm{Vdc}$ \\
\hline & GarrettCom & SWITCH, 8 PORT & 0464D & Switch 8 Port \\
\hline & Hitachi & $\mathrm{HDD}, 40 \mathrm{~GB}$ & & Hard Drive 40Gig \\
\hline & Hoffman & ENCLOSURE, REPEATER, MACHINED & $7049 \mathrm{R}$ & Repeater Enclosure (machined) \\
\hline & Hoffman & PANEL, 8X6 & 7055P & Panel 8X6 \\
\hline & Hoffman & UTILITY BOX, 8X6X4 & 7055B & Box 8X6X4 Utility \\
\hline & Hummel & GRIP, CORD, $1 / 2$ & 7305D & $1 / 2$ Cord Grip \\
\hline & Hummel & GRIP, CORD, $1 / 4$ & $7305 \mathrm{G}$ & $1 / 4$ Cord Grip \\
\hline & Hummel & GRIP, CORD, $3 / 4$ & $7305 \mathrm{~F}$ & 3/4 Cord Grip \\
\hline & IBase Technology & CONCENTRATOR & 0463A & Concentrator \\
\hline & Mean Well & CONVERTER, 12 VDC TO 5 VDC & $7499 B$ & $12 \mathrm{Vdc}$ to $5 \mathrm{Vdc}$ Converter \\
\hline & Mean Well & POWER SUPPLY, 7.5A 13.5 VDC & $7499 C$ & Power Supply $13.5 \mathrm{Vdc} 7.5 \mathrm{~A}$ \\
\hline & Minute Man & UPS, 2300 VA & 0475 & UPS 2300VA \\
\hline
\end{tabular}




\begin{tabular}{|c|c|c|c|}
\hline Manufacturer & Nomenclature & Part Number & Description \\
\hline Netgear & SWITCH, 16 PORT, ETHERNET & 0464G & Ethernet Switch 16 port \\
\hline PC CITY BUILD & WORK STATION, RAVEN & 0458 & Raven Work Station \\
\hline Pelco & ADAPTER, POLE & 0461B & Pole Adapter \\
\hline Pelco & DEFROSTER, 230 VAC & $0461 G$ & Defroster, 230Vac \\
\hline Pelco & HOUSING, CAMERA, 230 VAC & $0461 \mathrm{~F}$ & Camera Housing, 230Vac \\
\hline Pelco & HOUSING, CAMERA, 24 VAC & $0461 \mathrm{E}$ & Camera Housing, 24Vac \\
\hline Pelco & MOUNT, WALL & $0461 C$ & Wall Mount \\
\hline Pelco & POWER SUPPLY, CAMERA & 0462B & Camera Power Supply \\
\hline Pelco & POWER SUPPLY, CAMERA & $0462 \mathrm{~A}$ & Camera Power Supply \\
\hline Pelco & POWER SUPPLY, CAMERA & 0462 & Camera Power Supply \\
\hline Pelco & SUN SHIELD & $0461 \mathrm{H}$ & Sun Shroud \\
\hline PI & CABLE, POWER, IEC MALE TO FEMALE & $7303 B$ & Power Cord IEC M/F \\
\hline Quabbin Wire \& Cable & CABLE, 12 PAIR CROSSOVER, 1000 FT & 8525 & 12 Pair Crossover Cable $1000 \mathrm{ft}$ \\
\hline Seven Star & POWER STRIP, UNIVERSAL & 7303P & Power Strip Universal \\
\hline Sony & CAMERA & 0460 & Camera \\
\hline Titan Wireless & INJECTOR, POWER OVER ETHERNET & 0466B & PoE Injectors \\
\hline Tripp Lite & FAN, RACK MOUNT, FAN ROOF & 0459F & Rack Mount Fan Roof \\
\hline Tripp Lite & KIT, CABLE, KVM & 0459K1 & KVM Cable Kit 2 per switch \\
\hline Tripp Lite & RACK, ENCLOSED, 19 INCH & 0459R & 19" Enclosed Rack (22.75" High) \\
\hline Tripp Lite & SHELF, RACK MOUNT & 0459S & Rack Mount Shelf \\
\hline Tripp Lite & SHELF, RACK MOUNT, SLIDING & 0459SS & Rack Mount Sliding Shelf \\
\hline Tripp Lite & SWITCH, KVM & 0459K & KVM Switch \\
\hline Tripp Lite & UPS, 1500 VA, RACK OR TOWER & 0476A & UPS 1500VA RACK/TOWER \\
\hline Tripp Lite & UPS, 750 VA & 0476 & UPS 750 VA \\
\hline TSA & CABLE, 2 CONDUCTOR 18AWG, $100 \mathrm{FT}$ & & 2 Conductor \#18AWG 100ft \\
\hline TSA & CAMERA, NETWORK & $0460 C$ & SNC-CH140 NETWORK CAMERA \\
\hline TSA & CIRCUIT BREAKER & $7802 B$ & Circuit Breaker \\
\hline TSA & COMPACT FLASH, PEN DRIVE, WITH CABLE & 0463B & Pen Drive with cable \\
\hline TSA & MEDIA CONVERTER & 8590 & Magnum MEDIA CONVERTER \\
\hline
\end{tabular}




\begin{tabular}{|c|c|c|c|}
\hline Manufacturer & Nomenclature & Part Number & Description \\
\hline TSA & SOFTWARE, PC, RAVEN & & RAVEN Softeware for PC \\
\hline TSA & SOFTWARE, RAVEN, CONCENTRATOR & & RAVEN Software for Concentator \\
\hline TSA & TERMINAL STRIP & $2533 \mathrm{E}$ & Terminal Strip \\
\hline TSA & UPS, 1500VA & 0476A & 1500VA UPS \\
\hline SafePass CAS & CAS & & Communications \\
\hline Cisco & SERVER & & Cisco Server \\
\hline Cisco & SERVER & & Cisco Server \\
\hline Cisco & SWITCH, RACK MOUNT, 24 PORT & & 24 Port Rack Mount Switch \\
\hline Cisco & SWITCH, RACK MOUNT, 48 PORT & & 48 Port Rack Mount Switch \\
\hline Cisco & VPN APPLIANCE & & VPN Appliance \\
\hline GarrettCom & CONVERTER, MULTI MODE, WITH SC CONNECTOR & & Multi-Mode Converter w/ SC Connector \\
\hline GarrettCom & CONVERTER, SINGLE MODE, WITH SC CONNECTOR & & Single Mode Converter w/ SC Connector \\
\hline Hewlett Packard & PRINTER, WITH SCANNER AND FAX & & Printer/Scanner/Fax + Builtin Network Card \\
\hline Hewlett Packard & SERVER, 1 CPU 4GB RAM, 160GB & & 1CPU,4GB RAM,160GB HDD \\
\hline Hewlett Packard & SERVER, 1 CPU 4GB RAM, 250GB & & 1CPU,4GB RAM,250GB HDD \\
\hline Hewlett Packard & SERVER, 1 CPU 4GB RAM, 5X300 GB & & $1 \mathrm{CPU}, 4 \mathrm{~GB}$ RAM, $5 \times 300 \mathrm{~GB}$ HDD \\
\hline Hewlett Packard & SERVER, 2 CPU 4GB RAM, $5 \times 300$ GB & & 2 CPU, 4GB RAM, 5×300GB HDD \\
\hline Microsoft & KIT, KEYBOARD MOUSE & & For Use with Extender \\
\hline Microsoft & SOFTWARE, 5 CAL & & Based on $5 \mathrm{CAL}$ \\
\hline Microsoft & SOFTWARE, 5 CAL & & Based on $5 \mathrm{CAL}$ \\
\hline NEC & MONITOR, FLAT PANEL, 17 INCH & & For Use with Extender \\
\hline Netgear & SWITCH, 8 PORT & & 8 Port Desktop Switch \\
\hline Netgear & SWITCH, RACK MOUNT, 12 PORT & & 12 Port Rack Mount Switch \\
\hline Norton & SOFTWARE, BACKUP, GHOST & & Software Ghost Backup Software \\
\hline SoftwareFX & SOFTWARE, WINDOWS FORMS & & Windows Forms \\
\hline SuperLogics & KIT & & Upgraded RAM,CPU,HDD, OS, Vidcard, and Wall mount bracket \\
\hline Tripp Lite & CABLE & & Comes with KVM Cables \\
\hline Tripp Lite & CABLE, EXTENDER, KVM & & KVM extender \\
\hline Tripp Lite & KIT, CABLE, B020-008-17 CONSOLE & & Cable Kit for B020-008-17 Console \\
\hline
\end{tabular}




\begin{tabular}{|c|c|c|c|}
\hline Manufacturer & Nomenclature & Part Number & Description \\
\hline Tripp Lite & PATCH PANEL, 16 PORT & & 16 Port Patch Panel w/ SC Connectors \\
\hline Tripp Lite & PATCH PANEL, 24 PORT & & 24 Port Patch Panel \\
\hline Tripp Lite & RACK, $25 \mathrm{U}$ & & 25 U Rack \\
\hline Tripp Lite & SWITCH, 8 PORT KVM & & Built-in 8 Port KVM \\
\hline Tripp Lite & UPS, 1500VA & & 1500VA UPS \\
\hline Tripp Lite & UPS, 750VA & & 750VA UPS \\
\hline Schneider CAS & CAS & & Communications \\
\hline$* * *$ Unknown & MEDIA CONVERTER & $59190634-7$ & Media Converters \\
\hline APC & BATTERY PACK & JB0829023294 & APC Battery Pack \\
\hline APC & NETWORK CARD, MANAGEMENT & & APC Network Management Card \\
\hline APC & UPS, 1500 VA $120 \mathrm{~V}$ & & $\begin{array}{l}\text { Battery Backup / UPS RS } 1500 \text { LCD - UPS - } 865 \text { Watt - } 1500 \text { VA } 120 \text { V UPS } \\
\text { System }\end{array}$ \\
\hline Belkin & CABLE, CAT5E, RJ-45 & & BELKIN FastCAT5e RJ-45 Snagless Patch cable - $2.1 \mathrm{~m}$. or equal (cables) \\
\hline Cisco & TELEPHONY, VOIP & SPA901 & VolP Telephony \\
\hline Dell & CABLE, PRINTER, USB $10 \mathrm{FT}$ & $310-8688$ & DELL USB PRINTER CABLE- 10 FTBLACK \\
\hline Dell & KEYBOARD, USB & 310-7949 & Dell Entry Level USB Keyboard \\
\hline Dell & MONITOR, FLAT PANEL & 2209WA & Dell Flat Panel Monitor \\
\hline Dell & MONITOR, FLAT PANEL & 1908FP-BLK & Dell Flat Panel Monitor \\
\hline Dell & MOUSE, USB, OPTICAL & $310-9602$ & Dell USB 2 Button Optical Mouse \\
\hline Dell & PRINTER & GQ4BPG1 & Dell V305 Printer \\
\hline Dell & PRINTER, MULTIFUNCTION, COLOR LASER & $3115 \mathrm{CN}$ & Dell Multifunction Color Laser Printer \\
\hline Dell & SPEAKER, SOUND BAR & $313-6414$ & Dell Speakers AX510 black Sound Bar forUltraSharp Flat Panel Displays \\
\hline Dell & SWITCH, 24 PORT & & Dell PowerConnect 2724 24-port Switch \\
\hline Dell & SWITCH, 8 PORT, GBE WEB MANAGED & FPX9FH1 & $\begin{array}{l}\text { Dell PowerConnect/ PowerConnect 2708, } 8 \text { Port GbE Web-managed } \\
\text { Switch-R }\end{array}$ \\
\hline Dell & WORK STATION, MINITOWER & 1J3SNJ1 & Dell Computer Precision T5400 Mini Tower \\
\hline Exceltech & INVERTER, SINEWAVE & XP-125-12 & Exceltech Sinewave Inverter V \\
\hline Generic Manufacturer & CABLE, POWER, 3 FT & NA & $6 \mathrm{ft}$. (2) $3 \mathrm{ft}$. (2) power cords \\
\hline Generic Manufacturer & HUB, USB 2.0, 4 PORT & A1122455 & 4-Port USB 2.0 Plus Hub - White \\
\hline
\end{tabular}




\begin{tabular}{|c|c|c|c|}
\hline Manufacturer & Nomenclature & Part Number & Description \\
\hline Mean Well & INVERTER, POWER & TS200-124A & Meanwell Power Inverter \\
\hline Mean Well & UPS & DR-UPS-40 & Meanwell UPS Module \\
\hline Pelco & ADAPTER, POLE & PA101 & Pole adapter/pole mounts \\
\hline Pelco & \multicolumn{2}{|l|}{ ENCLOSURE, ENVIRONMENTAL } & Environmental Enclosure \\
\hline Pelco & $\begin{array}{l}\text { ENCLOSURE, ENVIRONMENTAL, HEATER AND } \\
\text { BLOWER }\end{array}$ & EH4718-2 & Enclosure Env Alum 18 in. 24VAC Htr\&Blwr \\
\hline Pelco & $\begin{array}{l}\text { LENS, IR CORRECTED AI DIRECT DRIVE, 1/3 INCH } 2.8 \\
\text { TO } 11 \mathrm{MM}\end{array}$ & 13VDIR2.8-1 1 & Lens $1 / 3$ in. $2.8-11 \mathrm{~mm}$ IR corrected AI Direct Drive \\
\hline Pelco & $\begin{array}{l}\text { LENS, IR CORRECTED AI DIRECT DRIVE, } 1 / 3 \text { INCH } 3 \\
\text { TO } 8.5 \mathrm{MM}\end{array}$ & 13VDIR3-8.5 & Lens $1 / 3$ in. 3-8.5mm IR corrected Al Direct Drive \\
\hline Pelco & MOUNT, WALL & EM22 & Medium-duty Wall Mt for EH4014 \& EH55 \\
\hline Pelco & POWER SUPPLY, 4A, 1 OUTPUT & WCS1-4 & Camera Power Supply 4Amp 1 Output \\
\hline Pelco & SUN SHIELD & SS4718 & Sun Shield for EH4718 series only \\
\hline Raytec & ILLUMINATOR, IR & RM25-30 & Raymax 25 IR Illuminators \\
\hline Raytec & ILLUMINATOR, IR & RM100-A1-50 & Raytec RayMax IR illuminator, 200 - 300 feet \\
\hline Sarix & CAMERA, NETWORK & AAM-CROO & Sarix Network Camera \\
\hline TSi Power & $\begin{array}{l}\text { VOLTAGE REGULATOR, OUTDOOR, } 172 \text { TO } 276 \text { VAC } \\
\text { INPUT }\end{array}$ & VRx-2100-8071 & 172-276 vac input precision regulator for outdoor installation \\
\hline \multicolumn{2}{|c|}{ Serco CAS - 1 Generation Old } & & Communications \\
\hline APC & UPS, 1500VA & varies & APC 1500 UPS. Exact model number is country/region specific. \\
\hline APC & UPS, 1500VA & varies & $\begin{array}{l}\text { APC } 1500 \text { UPS. Used at smaller sites. Large sites may also have } \\
\text { additional } 1500 \text { UPS and/or a } 3000 \text { UPS. Exact model number is } \\
\text { country/region specific. }\end{array}$ \\
\hline APC & UPS, 1500VA & varies & $\begin{array}{l}\text { APC } 1500 \text { UPS. Used at smaller sites. Large sites may also have } \\
\text { additional } 1500 \text { UPS and/or a } 3000 \text { UPS. Exact model number is } \\
\text { country/region specific. }\end{array}$ \\
\hline APC & UPS, 3000VA & varies & $\begin{array}{l}\text { APC } 3000 \text { UPS. This is not used at smaller sites. Large sites may also } \\
\text { have one or more } 1500 \text { UPS. Exact model number is country/region } \\
\text { specific. }\end{array}$ \\
\hline APC & UPS, 3000VA & varies & $\begin{array}{l}\text { APC } 3000 \text { UPS. This is not used at smaller sites. Large sites may also } \\
\text { have one or more } 1500 \text { UPS. Exact model number is country/region } \\
\text { specific. }\end{array}$ \\
\hline
\end{tabular}




\begin{tabular}{|c|c|c|c|}
\hline Manufacturer & Nomenclature & Part Number & Description \\
\hline APC & UPS, 5000VA & varies & $\begin{array}{l}\text { APC } 5000 \text { UPS. This is not used at smaller sites. Large sites may also } \\
\text { have one or more } 1500 \text { UPS. Exact model number is country/region } \\
\text { specific. }\end{array}$ \\
\hline Cisco & FIREWALL & varies & $\begin{array}{l}\text { Cisco ASA } 5505 \text { Firewall. Not used at all sites. Exact model is site/country } \\
\text { specific }\end{array}$ \\
\hline Cisco & ROUTER & varies & $\begin{array}{l}\text { Cisco } 2821 \text { Router. Not used at all sites. Exact model is site/country } \\
\text { specific }\end{array}$ \\
\hline Cisco & SWITCH, 24 PORT & varies & $\begin{array}{l}\text { Cisco } 2960 \text { - } 24 \text { port switch. Used at newer sites. Smaller sites may only } \\
\text { have this switch. Larger sites may also have additional 2960's and/or a } \\
3750-48 \text {. Exact model is site/country specific }\end{array}$ \\
\hline Cisco & SWITCH, 24 PORT & varies & $\begin{array}{l}\text { Cisco } 2950 \text { - } 24 \text { port switch. Used at older sites. Smaller sites may only } \\
\text { have this switch. Larger sites may also have additional 2950's and/or a } \\
\text { 3750-48. Exact model is site/country specific }\end{array}$ \\
\hline Cisco & SWITCH, 24 PORT & varies & $\begin{array}{l}\text { Cisco } 2960 \text { - } 24 \text { port switch. This is used at newer sites. Exact model is } \\
\text { site/country specific }\end{array}$ \\
\hline Cisco & SWITCH, 24 PORT & varies & $\begin{array}{l}\text { Cisco } 2960 \text { - } 24 \text { port switch. Used at newer sites. Smaller sites may only } \\
\text { have this switch. Larger sites may also have additional } 2960 \text { 's and/or a } \\
3750-48 \text {. Exact model is site/country specific }\end{array}$ \\
\hline Cisco & SWITCH, 24 PORT & varies & $\begin{array}{l}\text { Cisco } 2950 \text { - } 24 \text { port switch. Used at older sites. Smaller sites may only } \\
\text { have this switch. Larger sites may also have additional } 2950 \text { 's and/or a } \\
3750-48 \text {. Exact model is site/country specific }\end{array}$ \\
\hline Cisco & SWITCH, 48 PORT & varies & $\begin{array}{l}\text { Cisco } 3750 \text { - } 48 \text { port switch. This is not used at smaller sites. Large sites } \\
\text { may also have one or more } 2960-24 \text { or } 2950-24 \text {. Exact model is } \\
\text { site/country specific }\end{array}$ \\
\hline Cisco & SWITCH, 48 PORT & varies & $\begin{array}{l}\text { Cisco } 2950 \text { - } 24 \text { port switch. This was used at older sites. Exact model is } \\
\text { site/country specific }\end{array}$ \\
\hline Cisco & SWITCH, 48 PORT & varies & $\begin{array}{l}\text { Cisco } 3750 \text { - } 48 \text { port switch. This is not used at smaller sites. Large sites } \\
\text { may also have one or more } 2960-24 \text {. Exact model is site/country specific }\end{array}$ \\
\hline Hewlett Packard & CHASSIS, 650W 80 PLUS POWER SUPPLY & GM505AV & HP XW6600 650W 80 PLUS energy efficient chassis \\
\hline Hewlett Packard & CHASSIS, 650W 80 PLUS POWER SUPPLY & GM505AV & HP XW6600 650W 80 PLUS energy efficient chassis \\
\hline Hewlett Packard & CHASSIS, 650W 80 PLUS POWER SUPPLY & GM505AV & HP XW6600 650W 80 PLUS energy efficient chassis \\
\hline Hewlett Packard & CHASSIS, SERVER, PROLIANT & 391835-B21 & HP ProLiant DL380G5 Rack CTO Chassis \\
\hline Hewlett Packard & CHASSIS, SERVER, PROLIANT & 391835-B21 & HP ProLiant DL380G5 Rack CTO Chassis \\
\hline Hewlett Packard & CPU, XEON E5420, CPU 1 & 458577-L21 & $\begin{array}{l}\text { Quad-Core Intel }{ }^{\circledR} \text { Xeon }{ }^{\circledR} \text { Processor E5420 ( } 2.50 \text { GHz, } 80 \text { Watts, } 1333 \text { FSB) } \\
\text { DL380 FIO Kit (1st) }\end{array}$ \\
\hline Hewlett Packard & CPU, XEON E5420, CPU 1 & GW497AV & Intel Xeon 54202.50 12MB/1333 QC (1st) \\
\hline
\end{tabular}




\begin{tabular}{|c|c|c|c|}
\hline Manufacturer & Nomenclature & Part Number & Description \\
\hline Hewlett Packard & CPU, XEON E5420, CPU 1 & 458577-L21 & $\begin{array}{l}\text { Quad-Core Intel }{ }^{\circledR} \text { Xeon }^{\circledR} \text { Processor E5420 }(2.50 \text { GHz, } 80 \text { Watts, } 1333 \text { FSB) } \\
\text { DL380 FIO Kit (1st) }\end{array}$ \\
\hline Hewlett Packard & CPU, XEON E5420, CPU 1 & GW497AV & Intel Xeon 54202.50 12MB/1333 QC (1st) \\
\hline Hewlett Packard & CPU, XEON E5420, CPU 1 & GW497AV & Intel Xeon 54202.50 12MB/1333 QC (1st) \\
\hline Hewlett Packard & CPU, XEON E5420, CPU 2 & GW498AV & Intel Xeon 54202.50 12MB/1333 QC (2nd) \\
\hline Hewlett Packard & CPU, XEON E5420, CPU 2 & GW498AV & Intel Xeon 54202.50 12MB/1333 QC (2nd) \\
\hline Hewlett Packard & CPU, XEON E5420, CPU 2 & GW498AV & Intel Xeon 54202.50 12MB/1333 QC (2nd) \\
\hline Hewlett Packard & CPU, XEON E5420, CPU 2 & 458577-B21 & $\begin{array}{l}\text { Quad-Core Intel }{ }^{\circledR} \text { Xeon }^{\circledR} \text { Processor E5420 ( } 2.50 \text { GHz, } 80 \text { Watts, } 1333 \text { FSB) } \\
\text { (2nd) }\end{array}$ \\
\hline Hewlett Packard & CPU, XEON E5420, CPU 2 & 458577-B21 & $\begin{array}{l}\text { Quad-Core Intel }{ }^{\circledR} \text { Xeon }^{\circledR} \text { Processor E5420 }(2.50 \text { GHz, } 80 \text { Watts, } 1333 \text { FSB) } \\
\text { (2nd) }\end{array}$ \\
\hline Hewlett Packard & ENCLOSURE, DRIVE & AD542B & M5314B FC Drive Enclosure. SFI Sites and Taiwan. \\
\hline Hewlett Packard & ENCLOSURE, MSA, 60 SINGLE & 418408-B21 & $\begin{array}{l}\text { HP Storage Works Modular Storage Array (MSA) } 60 \text { single cabinet } \\
\text { enclosure. Not used at all sites. }\end{array}$ \\
\hline Hewlett Packard & HDD ARRAY & AD554B & EVA 40002 CID SAN. SFI Sites and Taiwan. \\
\hline Hewlett Packard & HDD CONTROLLER CARD & 381513-B21 & HP Smart Array P800/512 BBWC Controller (For newer PCle systems only) \\
\hline Hewlett Packard & HDD CONTROLLER CARD & 411508-B21 & HP Smart Array E200/128 BBWC Controller \\
\hline Hewlett Packard & HDD CONTROLLER CARD & 337972-B21 & $\begin{array}{l}\text { HP Smart Array P600/256 BBWC Controller (For older PCI-X systems } \\
\text { only) }\end{array}$ \\
\hline Hewlett Packard & HDD CONTROLLER CARD, BBWC, E200/128 & 411508-B21 & HP Smart Array E200/128 BBWC Controller \\
\hline Hewlett Packard & HDD CONTROLLER CARD, P600/256 & 337972-B21 & $\begin{array}{l}\text { HP Smart Array P600/256 BBWC Controller (For older PCI-X systems } \\
\text { only) }\end{array}$ \\
\hline Hewlett Packard & HDD CONTROLLER CARD, P800/512 & 381513-B21 & HP Smart Array P800/512 BBWC Controller (For newer PCle systems only) \\
\hline Hewlett Packard & HDD, FC 10K, 300GB & $364622-B 23$ & HDD 300Gb FC 10k \\
\hline Hewlett Packard & HDD, SAS 10K, 146GB & 507125-B21 & HP 146GB 6G SAS 10K SFF DP ENT HDD \\
\hline Hewlett Packard & HDD, SAS 10K, 146GB & 507125-B21 & HP 146GB 6G SAS 10K SFF DP ENT HDD \\
\hline Hewlett Packard & HDD, SAS 7.2K, 1TB & 461137-B21 & HP 1TB 7.2k DP MDL SAS 1y Wty HDD \\
\hline Hewlett Packard & HDD, SATA 7200, 250GB & GM539AV & 250 GB SATA NCQ 7200 (2nd) \\
\hline Hewlett Packard & HDD, SATA 7200, 250GB & GM539AV & 250 GB SATA NCQ 7200 (2nd) \\
\hline Hewlett Packard & HDD, SATA 7200, 250GB & GM539AV & 250 GB SATA NCQ 7200 (1st) \\
\hline Hewlett Packard & HDD, SATA 7200, 250GB & GM539AV & 250 GB SATA NCQ 7200 (2nd) \\
\hline
\end{tabular}




\begin{tabular}{|c|c|c|c|}
\hline Manufacturer & Nomenclature & Part Number & Description \\
\hline Hewlett Packard & HDD, SATA 7200, 250GB & GM539AV & 250 GB SATA NCQ 7200 (1st) \\
\hline Hewlett Packard & HDD, SATA 7200, 250GB & GM539AV & 250 GB SATA NCQ 7200 (1st) \\
\hline Hewlett Packard & INTERFACE ADAPTER & 262588-B21 & HP IP Console 1 pack interface adapter \\
\hline Hewlett Packard & KEYBOARD, PS2 & GM604AV\#ABA & HP PS/2 Standard Keyboard \\
\hline Hewlett Packard & KEYBOARD, PS2 & GM604AV\#ABA & HP PS/2 Standard Keyboard \\
\hline Hewlett Packard & KEYBOARD, PS2 & GM604AV\#ABA & HP PS/2 Standard Keyboard \\
\hline Hewlett Packard & KIT, LOCALIZATION & GM506AV\#ABA & HP XW6600 Localization Kit \\
\hline Hewlett Packard & KIT, LOCALIZATION & GM506AV\#ABA & HP XW6600 Localization Kit \\
\hline Hewlett Packard & KIT, LOCALIZATION & GM506AV\#ABA & HP XW6600 Localization Kit \\
\hline Hewlett Packard & $\begin{array}{l}\text { KIT, US RACKMOUNT, KEYBOARD MOUSE AND } \\
\text { MONITOR }\end{array}$ & AG052A & HP TFT7600 US Rackmount Keyboard, Mouse, 17 inch monitor \\
\hline Hewlett Packard & MEMORY KIT, DDR2-667, 4GB 4X1 & GM568AV & 4GB (4x1GB) DDR2-667 ECC FBD \\
\hline Hewlett Packard & MEMORY KIT, DDR2-667, 4GB 4X1 & GM568AV & 4GB (4x1GB) DDR2-667 ECC FBD \\
\hline Hewlett Packard & MEMORY KIT, DDR2-667, 4GB 4X1 & GM568AV & 4GB (4x1GB) DDR2-667 ECC FBD \\
\hline Hewlett Packard & MEMORY KIT, PC2-5300, 4GB 2X2 & 397413-B21 & 4 GB FBD PC2-5300 2 × 2 GB Dual Rank Kit \\
\hline Hewlett Packard & MEMORY KIT, PC2-5300, 4GB 2X2 & 397413-B21 & 4 GB FBD PC2-5300 2 × 2 GB Dual Rank Kit \\
\hline Hewlett Packard & MONITOR, FLAT PANEL, 17 INCH & L1706 & HP 17 inch LCD monitor \\
\hline Hewlett Packard & MONITOR, FLAT PANEL, 17 INCH & L1706 & HP 17 inch LCD monitor \\
\hline Hewlett Packard & MONITOR, FLAT PANEL, 19 INCH & L1906 & HP 19 inch LCD monitor \\
\hline Hewlett Packard & MONITOR, FLAT PANEL, 19 INCH & L1906 & HP 19 inch LCD monitor \\
\hline Hewlett Packard & MONITOR, FLAT PANEL, 20 INCH & EF227A4\#ABA & HP 20 inch LCD monitor \\
\hline Hewlett Packard & MONITOR, FLAT PANEL, 20 INCH & EF227A4\#ABA & HP 20 inch LCD monitor \\
\hline Hewlett Packard & MONITOR, FLAT PANEL, 20 INCH & EF227A4\#ABA & HP 20 inch LCD monitor \\
\hline Hewlett Packard & MOUSE, PS2 & GM598AV & HP PS/2 Scroll Mouse \\
\hline Hewlett Packard & MOUSE, PS2 & GM598AV & HP PS/2 Scroll Mouse \\
\hline Hewlett Packard & MOUSE, PS2 & GM598AV & HP PS/2 Scroll Mouse \\
\hline Hewlett Packard & NETWORK CARD, PCIE, DUAL PORT GIGABIT & 394795-B21 & HP NC380T PCI Express Dual Port Multifunction Gigabit Server Adapter \\
\hline Hewlett Packard & NETWORK CARD, PCIE, DUAL PORT GIGABIT & 394795-B21 & HP NC380T PCI Express Dual Port Multifunction Gigabit Server Adapter \\
\hline Hewlett Packard & OPTICAL DRIVE, SATA, 16X DVD-ROM & GM577AV & HP 16X DVD-ROM \\
\hline
\end{tabular}




\begin{tabular}{|c|c|c|c|}
\hline Manufacturer & Nomenclature & Part Number & Description \\
\hline Hewlett Packard & OPTICAL DRIVE, SATA, 16X DVD-ROM & GM577AV & HP 16X DVD-ROM \\
\hline Hewlett Packard & OPTICAL DRIVE, SATA, 16X DVD-ROM & GM577AV & HP 16X DVD-ROM \\
\hline Hewlett Packard & OPTICAL DRIVE, SATA, 8X 24X DVD-ROM & 264007-B21 & Slimline DVD-ROM Drive (8x/24x) Option Kit \\
\hline Hewlett Packard & OPTICAL DRIVE, SATA, 8X 24X DVD-ROM & 264007-B21 & Slimline DVD-ROM Drive (8x/24x) Option Kit \\
\hline Hewlett Packard & POWER SUPPLY, 350/370/380 & 399771-B21 & Redundant Power Supply 350/370/380 G5 Kit \\
\hline Hewlett Packard & POWER SUPPLY, 350/370/380 & 399771-B21 & Redundant Power Supply 350/370/380 G5 Kit \\
\hline Hewlett Packard & SOFTWARE, DOWNGRADE TO XP 32 BIT & GR528AV\#ABA & Microsoft Vista 32 bit downgrade to XP 32 bit \\
\hline Hewlett Packard & SOFTWARE, DOWNGRADE TO XP 32 BIT & GR528AV\#ABA & Microsoft Vista 32 bit downgrade to XP 32 bit \\
\hline Hewlett Packard & SOFTWARE, DOWNGRADE TO XP 32 BIT & GR528AV\#ABA & Microsoft Vista 32 bit downgrade to XP 32 bit \\
\hline Hewlett Packard & SPEAKER, SOUND BAR & EE418AA & HP 2T flat panel speaker bar \\
\hline Hewlett Packard & SPEAKER, SOUND BAR & EE418AA & HP 2T flat panel speaker bar \\
\hline Hewlett Packard & SPEAKER, SOUND BAR & EE418AA & HP 2T flat panel speaker bar \\
\hline Hewlett Packard & SWITCH, CAT5 KVM, 0X2X16 & $336045-B 21$ & HP CAT5 0x2×16 KVM Console Switch \\
\hline Hewlett Packard & SWITCH, SAN, 4/8 BASE & A7984A & Storage Works $4 / 8$ Base SAN Switch. SFI Sites and Taiwan. \\
\hline Hewlett Packard & TAPE DRIVE, MSL & BL531A & HP Storage Workds MSL 2024 Tape Backup Drive. Not used at all sites. \\
\hline Hewlett Packard & VIDEO CARD, NVIDIA QUADRO, 256MB & GN493AV & NVIDIA Quadro NVS290 256 MB PCle \\
\hline Hewlett Packard & VIDEO CARD, NVIDIA QUADRO, 256MB & GN493AV & NVIDIA Quadro NVS290 256 MB PCle \\
\hline Hewlett Packard & VIDEO CARD, NVIDIA QUADRO, 256MB & GN493AV & NVIDIA Quadro NVS290 256 MB PCle \\
\hline Hewlett Packard & WORK STATION & RV725AV & HP XW6600 Workstation Base Unit \\
\hline Hewlett Packard & WORK STATION & RV725AV & HP XW6600 Workstation Base Unit \\
\hline Hewlett Packard & WORK STATION & RV725AV & HP XW6600 Workstation Base Unit \\
\hline Serco CAS - 2 Generati & CAS & & Communications \\
\hline APC & UPS, 1500VA & varies & $\begin{array}{l}\text { APC } 1500 \text { UPS. Used at smaller sites. Large sites may also have } \\
\text { additional } 1500 \text { UPS and/or a } 3000 \text { UPS. Exact model number is } \\
\text { country/region specific. }\end{array}$ \\
\hline APC & UPS, 1500VA & varies & $\begin{array}{l}\text { APC } 1500 \text { UPS. Used at smaller sites. Large sites may also have } \\
\text { additional } 1500 \text { UPS and/or a } 3000 \text { UPS. Exact model number is } \\
\text { country/region specific. }\end{array}$ \\
\hline APC & UPS, 3000VA & varies & $\begin{array}{l}\text { APC } 3000 \text { UPS. This is not used at smaller sites. Large sites may also } \\
\text { have one or more } 1500 \text { UPS. Exact model number is country/region } \\
\text { specific. }\end{array}$ \\
\hline
\end{tabular}




\begin{tabular}{|c|c|c|c|}
\hline Manufacturer & Nomenclature & Part Number & Description \\
\hline APC & UPS, 3000VA & varies & $\begin{array}{l}\text { APC } 3000 \text { UPS. This is not used at smaller sites. Large sites may also } \\
\text { have one or more } 1500 \text { UPS. Exact model number is country/region } \\
\text { specific. }\end{array}$ \\
\hline APC & UPS, 5000VA & varies & $\begin{array}{l}\text { APC } 5000 \text { UPS. This is not used at smaller sites. Large sites may also } \\
\text { have one or more } 1500 \text { UPS. Exact model number is country/region } \\
\text { specific. }\end{array}$ \\
\hline Cisco & FIREWALL & varies & $\begin{array}{l}\text { Cisco ASA } 5505 \text { Firewall. Not used at all sites. Exact model is site/country } \\
\text { specific }\end{array}$ \\
\hline Cisco & ROUTER & varies & $\begin{array}{l}\text { Cisco } 2821 \text { Router. Not used at all sites. Exact model is site/country } \\
\text { specific }\end{array}$ \\
\hline Cisco & SWITCH, 24 PORT & varies & $\begin{array}{l}\text { Cisco } 2950 \text { - } 24 \text { port switch. Used at older sites. Smaller sites may only } \\
\text { have this switch. Larger sites may also have additional } 2950 \text { 's and/or a } \\
3750-48 \text {. Exact model is site/country specific }\end{array}$ \\
\hline Cisco & SWITCH, 24 PORT & varies & $\begin{array}{l}\text { Cisco } 2950 \text { - } 24 \text { port switch. Used at older sites. Smaller sites may only } \\
\text { have this switch. Larger sites may also have additional 2950's and/or a } \\
3750-48 \text {. Exact model is site/country specific }\end{array}$ \\
\hline Cisco & SWITCH, 48 PORT & varies & $\begin{array}{l}\text { Cisco } 3750-48 \text { port switch. This is not used at smaller sites. Large sites } \\
\text { may also have one or more } 2950-24 \text {. Exact model is site/country specific }\end{array}$ \\
\hline Cisco & SWITCH, 48 PORT & varies & $\begin{array}{l}\text { Cisco } 3750-48 \text { port switch. This is not used at smaller sites. Large sites } \\
\text { may also have one or more } 2950-24 \text {. Exact model is site/country specific }\end{array}$ \\
\hline ELO & MONITOR, TOUCH & ELO 1727 & ELO 1727 Touch LCD 3000 Series \\
\hline Hewlett Packard & CHASSIS, SERVER, PROLIANT & $371293-405$ & HP ProLiant DL380G4 U320 SCSI Rack Chassis \\
\hline Hewlett Packard & CHASSIS, SERVER, PROLIANT & $371293-405$ & HP ProLiant DL380G4 U320 SCSI Rack Chassis \\
\hline Hewlett Packard & $\mathrm{CPU}, \mathrm{X} 2.8$ & 397645-B21 & Intel X2.8-2MB/800MHz DL380 G4 FIO BASE (2nd) \\
\hline Hewlett Packard & $\mathrm{CPU}, \mathrm{X} 2.8$ & 397645-B21 & Intel X2.8-2MB/800MHz DL380 G4 FIO BASE (2nd) \\
\hline Hewlett Packard & $\mathrm{CPU}, \mathrm{X} 2.8$ & 397645-L22 & Intel X2.8-2MB/800MHz DL380 G4 FIO BASE (1st) \\
\hline Hewlett Packard & $\mathrm{CPU}, \mathrm{X} 2.8$ & 397645-L22 & Intel X2.8-2MB/800MHz DL380 G4 FIO BASE (1st) \\
\hline Hewlett Packard & CPU, XEON 5110, CPU 1 & EX617AV & Intel Xeon 51101.60 4MB/1066 DC (1st) \\
\hline Hewlett Packard & CPU, XEON 5110, CPU 1 & EX617AV & Intel Xeon 51101.60 4MB/1066 DC (1st) \\
\hline Hewlett Packard & CPU, XEON 5110, CPU 2 & EX618AV & Intel Xeon 51101.60 4MB/1066 DC (2nd) \\
\hline Hewlett Packard & CPU, XEON 5110, CPU 2 & EX618AV & Intel Xeon 51101.60 4MB/1066 DC (2nd) \\
\hline Hewlett Packard & ENCLOSURE, MSA, 60 SINGLE & 418408-B21 & $\begin{array}{l}\text { HP Storage Works Modular Storage Array (MSA) } 60 \text { single cabinet } \\
\text { enclosure. Not used at all sites. }\end{array}$ \\
\hline Hewlett Packard & FLOPPY DRIVE & ER242AV & 1.44 Mb Floppy Disk Drive \\
\hline
\end{tabular}




\begin{tabular}{|c|c|c|c|}
\hline Manufacturer & Nomenclature & Part Number & Description \\
\hline Hewlett Packard & FLOPPY DRIVE & ER242AV & 1.44 Mb Floppy Disk Drive \\
\hline Hewlett Packard & HDD CONTROLLER CARD & 291967-B21 & Smart Array 642 Controller \\
\hline Hewlett Packard & HDD CONTROLLER CARD & 291967-B21 & Smart Array 642 Controller \\
\hline Hewlett Packard & HDD, SAS 10K, 146GB & 286716-B22 & HP 146GB U320 10K Universal HDD \\
\hline Hewlett Packard & HDD, SAS 10K, 146GB & 286716-B22 & HP 146GB U320 10K Universal HDD \\
\hline Hewlett Packard & HDD, SAS 7.2K, 1TB & 461137-B21 & HP 1TB 7.2k DP MDL SAS 1y Wty HDD \\
\hline Hewlett Packard & HDD, SATA 7200, 80GB & ER259AV & 80 Gb SATA 3Gb/s 7200 (2nd) \\
\hline Hewlett Packard & HDD, SATA 7200, 80GB & ER259AV & 80 Gb SATA 3Gb/s 7200 (2nd) \\
\hline Hewlett Packard & HDD, SATA 7200, 80GB & ER249AV & 80 Gb SATA 3Gb/s 7200 (1st) \\
\hline Hewlett Packard & HDD, SATA 7200, 80GB & ER249AV & 80 Gb SATA 3Gb/s 7200 (1st) \\
\hline Hewlett Packard & KEYBOARD, PS2 & DZ204AV\#ABA & HP PS/2 STANDARD KEYBOARD \\
\hline Hewlett Packard & KEYBOARD, PS2 & DZ204AV\#ABA & HP PS/2 STANDARD KEYBOARD \\
\hline Hewlett Packard & KIT, LOCALIZATION & ER296AV\#ABA & HP XW6400 Localization Kit \\
\hline Hewlett Packard & KIT, LOCALIZATION & ER296AV\#ABA & HP XW6400 Localization Kit \\
\hline Hewlett Packard & MEMORY KIT, DDR2 PC2300, 4GB 2X2 & 343057-B21 & $\begin{array}{l}4 \text { GB of Advanced PC2 PC3200 DDR2 SDRAM DIMM Memory Kit ( } 2 \text { × } 2048 \\
\text { MB) }\end{array}$ \\
\hline Hewlett Packard & MEMORY KIT, DDR2 PC2300, 4GB 2X2 & 343057-B21 & $\begin{array}{l}4 \text { GB of Advanced PC2 PC3200 DDR2 SDRAM DIMM Memory Kit }(2 \times 2048 \\
\text { MB) }\end{array}$ \\
\hline Hewlett Packard & MONITOR, FLAT PANEL, 17 INCH & L1706 & HP 17 inch LCD monitor \\
\hline Hewlett Packard & MONITOR, FLAT PANEL, 19 INCH & L1906 & HP 19 inch LCD monitor \\
\hline Hewlett Packard & MONITOR, FLAT PANEL, 20 INCH & EF227A4\#ABA & HP 20 inch LCD monitor \\
\hline Hewlett Packard & MOUSE, PS2 & AA360AV & HP PS/2 SCROLL MOUSE \\
\hline Hewlett Packard & MOUSE, PS2 & AA360AV & HP PS/2 SCROLL MOUSE \\
\hline Hewlett Packard & NETWORK CARD, PCI-X, GIGABIT & 290563-B21 & HP NC7771 PCI-X 1000T Gigabit Server Adapter \\
\hline Hewlett Packard & NETWORK CARD, PCI-X, GIGABIT & 290563-B21 & HP NC7771 PCI-X 1000T Gigabit Server Adapter \\
\hline Hewlett Packard & OPTICAL DRIVE, DVDROM CDR & ER272AV & DVD-ROM/CDR combo \\
\hline Hewlett Packard & OPTICAL DRIVE, DVDROM CDR & ER272AV & DVD-ROM/CDR combo \\
\hline Hewlett Packard & OPTICAL DRIVE, SATA, 8X 24X DVD-ROM & 264007-B21 & Slimline DVD-ROM Drive (8x/24x) Option Kit \\
\hline Hewlett Packard & OPTICAL DRIVE, SATA, DVD-ROM CD-R & 264007-B21 & Slimline DVD-ROM Drive (8x/24x) Option Kit \\
\hline Hewlett Packard & POWER SUPPLY, AC REDUNDANT, HOT PLUG & $355892-001$ & Hot Plug AC Redundant Power Supply Module \\
\hline
\end{tabular}




\begin{tabular}{|c|c|c|c|}
\hline Manufacturer & Nomenclature & Part Number & Description \\
\hline Hewlett Packard & POWER SUPPLY, AC REDUNDANT, HOT PLUG & $355892-001$ & Hot Plug AC Redundant Power Supply Module \\
\hline Hewlett Packard & SPEAKER, SOUND BAR & EE418AA & HP 2T flat panel speaker bar \\
\hline Hewlett Packard & TAPE DRIVE, MSL & BL531A & HP Storage Workds MSL 2024 Tape Backup Drive. Not used at all sites. \\
\hline Hewlett Packard & VIDEO CARD, NVIDIA QUADRO, 128MB & RD064AV & NVIDIA Quadro NVS 285128 Mb PCle \\
\hline Hewlett Packard & VIDEO CARD, NVIDIA QUADRO, 128MB & RD064AV & NVIDIA Quadro NVS $285128 \mathrm{Mb}$ PCle \\
\hline Hewlett Packard & WORK STATION & ER236AV & HP XW6400 Workstation Base Unit \\
\hline Hewlett Packard & WORK STATION & ER236AV & HP XW6400 Workstation Base Unit \\
\hline \multirow[t]{2}{*}{ Serco CAS - 3 Generat } & CAS & & Communications \\
\hline & UPS, 1500VA & varies & $\begin{array}{l}\text { APC } 1500 \text { UPS. Used at smaller sites. Large sites may also have } \\
\text { additional } 1500 \text { UPS and/or a } 3000 \text { UPS. Exact model number is } \\
\text { country/region specific. }\end{array}$ \\
\hline APC & UPS, 1500VA & varies & $\begin{array}{l}\text { APC } 1500 \text { UPS. Used at smaller sites. Large sites may also have } \\
\text { additional } 1500 \text { UPS and/or a } 3000 \text { UPS. Exact model number is } \\
\text { country/region specific. }\end{array}$ \\
\hline APC & UPS, 3000VA & varies & $\begin{array}{l}\text { APC } 3000 \text { UPS. This is not used at smaller sites. Large sites may also } \\
\text { have one or more } 1500 \text { UPS. Exact model number is country/region } \\
\text { specific. }\end{array}$ \\
\hline APC & UPS, 3000VA & varies & $\begin{array}{l}\text { APC } 3000 \text { UPS. This is not used at smaller sites. Large sites may also } \\
\text { have one or more } 1500 \text { UPS. Exact model number is country/region } \\
\text { specific. }\end{array}$ \\
\hline APC & UPS, 5000VA & varies & $\begin{array}{l}\text { APC } 5000 \text { UPS. This is not used at smaller sites. Large sites may also } \\
\text { have one or more } 1500 \text { UPS. Exact model number is country/region } \\
\text { specific. }\end{array}$ \\
\hline Cisco & ROUTER & varies & $\begin{array}{l}\text { Cisco } 2821 \text { Router. Not used at all sites. Exact model is site/country } \\
\text { specific }\end{array}$ \\
\hline Cisco & SWITCH, 24 PORT & varies & $\begin{array}{l}\text { Cisco } 2950 \text { - } 24 \text { port switch. Used at older sites. Smaller sites may only } \\
\text { have this switch. Larger sites may also have additional 2950's and/or a } \\
3750-48 \text {. Exact model is site/country specific }\end{array}$ \\
\hline Cisco & SWITCH, 24 PORT & varies & $\begin{array}{l}\text { Cisco } 2950 \text { - } 24 \text { port switch. Used at older sites. Smaller sites may only } \\
\text { have this switch. Larger sites may also have additional 2950's and/or a } \\
3750-48 \text {. Exact model is site/country specific }\end{array}$ \\
\hline Cisco & SWITCH, 48 PORT & varies & $\begin{array}{l}\text { Cisco } 3750 \text { - } 48 \text { port switch. This is not used at smaller sites. Large sites } \\
\text { may also have one or more } 2950-24 \text {. Exact model is site/country specific }\end{array}$ \\
\hline Cisco & SWITCH, 48 PORT & varies & $\begin{array}{l}\text { Cisco } 3750 \text { - } 48 \text { port switch. This is not used at smaller sites. Large sites } \\
\text { may also have one or more } 2950-24 \text {. Exact model is site/country specific }\end{array}$ \\
\hline ELO & MONITOR, TOUCH & ELO 1727 & ELO 1727 Touch LCD 3000 Series \\
\hline
\end{tabular}




\begin{tabular}{|c|c|c|c|}
\hline Manufacturer & Nomenclature & Part Number & Description \\
\hline Hewlett Packard & CHASSIS, SERVER, PROLIANT & $371293-405$ & HP ProLiant DL380G4 U320 SCSI Rack Chassis \\
\hline Hewlett Packard & CHASSIS, SERVER, PROLIANT & $371293-405$ & HP ProLiant DL380G4 U320 SCSI Rack Chassis \\
\hline Hewlett Packard & CPU, X2.8 & 397645-L22 & Intel X2.8-2MB/800MHz DL380 G4 FIO BASE (1st) \\
\hline Hewlett Packard & CPU, X2.8 & 397645-B21 & Intel X2.8-2MB/800MHz DL380 G4 FIO BASE (2nd) \\
\hline Hewlett Packard & CPU, X2.8 & 397645-L22 & Intel X2.8-2MB/800MHz DL380 G4 FIO BASE (1st) \\
\hline Hewlett Packard & CPU, X2.8 & 397645-B21 & Intel X2.8-2MB/800MHz DL380 G4 FIO BASE (2nd) \\
\hline Hewlett Packard & CPU, XEON, 3.6GHZ & DY255AV & Intel Xeon $3.60 \mathrm{GHz} / 1 \mathrm{MB} 800 \mathrm{FSB}$ \\
\hline Hewlett Packard & CPU, XEON, 3.6GHZ & DY255AV & Intel Xeon $3.60 \mathrm{GHz} / 1 \mathrm{MB} 800 \mathrm{FSB}$ \\
\hline Hewlett Packard & ENCLOSURE, MSA, 60 SINGLE & 418408-B21 & $\begin{array}{l}\text { HP Storage Works Modular Storage Array (MSA) } 60 \text { single cabinet } \\
\text { enclosure. Not used at all sites. }\end{array}$ \\
\hline Hewlett Packard & FLOPPY DRIVE & DY290AV & FLOPPY DISK DRIVE \\
\hline Hewlett Packard & FLOPPY DRIVE & DY290AV & FLOPPY DISK DRIVE \\
\hline Hewlett Packard & HDD CONTROLLER CARD & DZ540AV & U320 SCSI CONTROLLER - RAID 0-1, EXTERNAL CONNECTOR \\
\hline Hewlett Packard & HDD CONTROLLER CARD & DZ540AV & U320 SCSI CONTROLLER - RAID 0-1, EXTERNAL CONNECTOR \\
\hline Hewlett Packard & HDD CONTROLLER CARD & 291967-B21 & Smart Array 642 Controller \\
\hline Hewlett Packard & HDD CONTROLLER CARD & 291967-B21 & Smart Array 642 Controller \\
\hline Hewlett Packard & HDD, SAS 10K, 146GB & 286716-B22 & HP 146GB U320 10K Universal HDD \\
\hline Hewlett Packard & HDD, SAS 10K, 146GB & 286716-B22 & HP 146GB U320 10K Universal HDD \\
\hline Hewlett Packard & HDD, SAS 7.2K, 1TB & 461137-B21 & HP 1TB 7.2k DP MDL SAS 1y Wty HDD \\
\hline Hewlett Packard & HDD, SCSI 15K, 36GB & DY277AV & 36GB U320 SCSI 15K (first) \\
\hline Hewlett Packard & HDD, SCSI 15K, 36GB & DY277AV & 36GB U320 SCSI 15K (second) \\
\hline Hewlett Packard & HDD, SCSI 15K, 36GB & DY277AV & 36GB U320 SCSI 15K (second) \\
\hline Hewlett Packard & HDD, SCSI 15K, 36GB & DY277AV & 36GB U320 SCSI 15K (first) \\
\hline Hewlett Packard & KEYBOARD, PS2 & DZ204AV\#ABA & HP PS/2 STANDARD KEYBOARD \\
\hline Hewlett Packard & KEYBOARD, PS2 & DZ204AV\#ABA & HP PS/2 STANDARD KEYBOARD \\
\hline Hewlett Packard & KIT, LOCALIZATION & DY250AV\#ABA & HP XW6200 LOCALIZATION KIT \\
\hline Hewlett Packard & KIT, LOCALIZATION & DY250AV\#ABA & HP XW6200 LOCALIZATION KIT \\
\hline Hewlett Packard & MEMORY KIT, DDR2 PC2300, 4GB 2X2 & 343057-B21 & $\begin{array}{l}4 \text { GB of Advanced PC2 PC3200 DDR2 SDRAM DIMM Memory Kit }(2 \times 2048 \\
\text { MB) }\end{array}$ \\
\hline
\end{tabular}




\begin{tabular}{|c|c|c|c|}
\hline Manufacturer & Nomenclature & Part Number & Description \\
\hline Hewlett Packard & MEMORY KIT, DDR2 PC2300, 4GB 2X2 & 343057-B21 & $\begin{array}{l}4 \text { GB of Advanced PC2 PC3200 DDR2 SDRAM DIMM Memory Kit }(2 \times 2048 \\
\text { MB) }\end{array}$ \\
\hline Hewlett Packard & MEMORY KIT, DDR2-400, 2GB 2X1 & DY288AV & 2GB (2×1GB) DDR2-400 ECC reg \\
\hline Hewlett Packard & MEMORY KIT, DDR2-400, 2GB 2X1 & DY288AV & $2 \mathrm{~GB}(2 \times 1 \mathrm{~GB}) \mathrm{DDR} 2-400 \mathrm{ECC}$ reg \\
\hline Hewlett Packard & MONITOR, FLAT PANEL, 17 INCH & L1706 & HP 17 inch LCD monitor \\
\hline Hewlett Packard & MONITOR, FLAT PANEL, 17 INCH & PX849A8\#ABA & L1706 17" FLAT PANEL DISPLAY \\
\hline Hewlett Packard & MONITOR, FLAT PANEL, 19 INCH & L1906 & HP 19 inch LCD monitor \\
\hline Hewlett Packard & MOUSE, PS2 & AA360AV & HP PS/2 SCROLL MOUSE \\
\hline Hewlett Packard & MOUSE, PS2 & AA360AV & HP PS/2 SCROLL MOUSE \\
\hline Hewlett Packard & NETWORK CARD, PCI-X, GIGABIT & 290563-B21 & HP NC7771 PCI-X 1000T Gigabit Server Adapter \\
\hline Hewlett Packard & NETWORK CARD, PCI-X, GIGABIT & 290563-B21 & HP NC7771 PCI-X 1000T Gigabit Server Adapter \\
\hline Hewlett Packard & OPTICAL DRIVE, DVDROM CDRW, $48 \mathrm{X}$ & DY295AV & 48X DVD-ROM/CDRW COMBO \\
\hline Hewlett Packard & OPTICAL DRIVE, DVDROM CDRW, $48 \mathrm{X}$ & DY295AV & 48X DVD-ROM/CDRW COMBO \\
\hline Hewlett Packard & OPTICAL DRIVE, SATA, 8X 24X DVD-ROM & 264007-B21 & Slimline DVD-ROM Drive (8x/24x) Option Kit \\
\hline Hewlett Packard & OPTICAL DRIVE, SATA, 8X 24X DVD-ROM & 264007-B21 & Slimline DVD-ROM Drive (8x/24x) Option Kit \\
\hline Hewlett Packard & POWER SUPPLY, AC REDUNDANT, HOT PLUG & 355892-001 & Hot Plug AC Redundant Power Supply Module \\
\hline Hewlett Packard & POWER SUPPLY, AC REDUNDANT, HOT PLUG & 355892-001 & Hot Plug AC Redundant Power Supply Module \\
\hline Hewlett Packard & TAPE DRIVE, MSL & BL531A & HP Storage Workds MSL 2024 Tape Backup Drive. Not used at all sites. \\
\hline Hewlett Packard & VIDEO CARD, NVIDIA QUADRO, 128MB & EE058AV & NVIDIA QUADRO NVS 285128 TC PCI-E \\
\hline Hewlett Packard & VIDEO CARD, NVIDIA QUADRO, 128MB & EE058AV & NVIDIA QUADRO NVS 285128 TC PCI-E \\
\hline Hewlett Packard & WORK STATION & DU937AV & HP XW6200 Workstation Base Unit \\
\hline Hewlett Packard & WORK STATION & DU937AV & HP XW6200 Workstation Base Unit \\
\hline Serco CAS - Current & CAS & & Communications \\
\hline APC & UPS, 1500VA & $\begin{array}{l}1500 \text { (exact model } \\
\text { country/region specific) }\end{array}$ & APC 1500 UPS. Exact model number is country/region specific. \\
\hline APC & UPS, 1500VA & $\begin{array}{l}1500 \text { (exact model } \\
\text { country/region specific) }\end{array}$ & $\begin{array}{l}\text { APC } 1500 \text { UPS. Used at smaller sites. Large sites may also have } \\
\text { additional } 1500 \text { UPS and/or a } 3000 \text { UPS. Exact model number is } \\
\text { country/region specific. }\end{array}$ \\
\hline APC & UPS, 1500VA & $\begin{array}{l}1500 \text { (exact model } \\
\text { country/region specific) }\end{array}$ & $\begin{array}{l}\text { APC } 1500 \text { UPS. Used at smaller sites. Large sites may also have } \\
\text { additional } 1500 \text { UPS and/or a } 3000 \text { UPS. Exact model number is } \\
\text { country/region specific. }\end{array}$ \\
\hline
\end{tabular}




\begin{tabular}{|c|c|c|c|}
\hline Manufacturer & Nomenclature & Part Number & Description \\
\hline APC & UPS, 3000VA & $\begin{array}{l}3000 \text { (exact model } \\
\text { country/region specific) }\end{array}$ & $\begin{array}{l}\text { APC } 3000 \text { UPS. This is not used at smaller sites. Large sites may also } \\
\text { have one or more } 1500 \text { UPS. Exact model number is country/region } \\
\text { specific. }\end{array}$ \\
\hline APC & UPS, 3000VA & $\begin{array}{l}3000 \text { (exact model } \\
\text { country/region specific) }\end{array}$ & $\begin{array}{l}\text { APC } 3000 \text { UPS. This is not used at smaller sites. Large sites may also } \\
\text { have one or more } 1500 \text { UPS. Exact model number is country/region } \\
\text { specific. }\end{array}$ \\
\hline APC & UPS, 5000VA & $\begin{array}{l}5000 \text { (exact model } \\
\text { country/region specific) }\end{array}$ & $\begin{array}{l}\text { APC } 5000 \text { UPS. This is not used at smaller sites. Large sites may also } \\
\text { have one or more } 1500 \text { UPS. Exact model number is country/region } \\
\text { specific. }\end{array}$ \\
\hline Cisco & FIREWALL, ASA 5505 & ASA 5505 & $\begin{array}{l}\text { Cisco ASA } 5505 \text { Firewall. Not used at all sites. Exact model is site/country } \\
\text { specific }\end{array}$ \\
\hline Cisco & ROUTER, 2821 & 2821 & $\begin{array}{l}\text { Cisco } 2821 \text { Router. Not used at all sites. Exact model is site/country } \\
\text { specific }\end{array}$ \\
\hline Cisco & SWITCH, 24 PORT & $2960-24$ & Cisco $2960-24$ port switch. Exact model is site/country specific \\
\hline Cisco & SWITCH, 24 PORT & $2960-24$ & $\begin{array}{l}\text { Cisco } 2960 \text { - } 24 \text { port switch. Smaller sites may only have this switch. } \\
\text { Larger sites may also have additional } 2960 \text { 's and/or a } 3750-48 \text {. Exact } \\
\text { model is site/country specific }\end{array}$ \\
\hline Cisco & SWITCH, 24 PORT & $2960-24$ & $\begin{array}{l}\text { Cisco } 2960 \text { - } 24 \text { port switch. Smaller sites may only have this switch. } \\
\text { Larger sites may also have additional } 2960 \text { 's and/or a } 3750-48 \text {. Exact } \\
\text { model is site/country specific }\end{array}$ \\
\hline Cisco & SWITCH, 48 PORT & $3750-48$ & $\begin{array}{l}\text { Cisco } 3750 \text { - } 48 \text { port switch. This is not used at smaller sites. Large sites } \\
\text { may also have one or more } 2960-24 \text {. Exact model is site/country specific }\end{array}$ \\
\hline Cisco & SWITCH, 48 PORT & $3750-48$ & $\begin{array}{l}\text { Cisco } 3750 \text { - } 48 \text { port switch. This is not used at smaller sites. Large sites } \\
\text { may also have one or more } 2960-24 \text {. Exact model is site/country specific }\end{array}$ \\
\hline Hewlett Packard & ADAPTER, INTERFACE, IP CONSOLE & & HP IP Console 1 pack interface adapter \\
\hline Hewlett Packard & CHASSIS, 650W 85\% EFFICIENCY POWER SUPPLY & & HP Z600 650W 85\% efficiency chassis \\
\hline Hewlett Packard & CHASSIS, 650W 85\% EFFICIENCY POWER SUPPLY & & HP Z600 650W 85\% efficiency chassis \\
\hline Hewlett Packard & CHASSIS, 650W 85\% EFFICIENCY POWER SUPPLY & & HP Z600 650W 85\% efficiency chassis \\
\hline Hewlett Packard & CHASSIS, SERVER, PROLIANT & DL380G6 & HP Proliant Server DL380 G6 Chassis \\
\hline Hewlett Packard & CHASSIS, SERVER, PROLIANT & DL380G6 & HP Proliant Server DL380 G6 Chassis \\
\hline Hewlett Packard & CPU, XEON E 5504, CPU 1 & E5504 & Intel Xeon E5504 2.004 MB/800 QC CPU-1 \\
\hline Hewlett Packard & CPU, XEON E 5504, CPU 2 & E5504 & Intel Xeon E5504 2.004 MB/800 QC CPU-2 \\
\hline Hewlett Packard & CPU, XEON E5504, CPU 1 & E5504 & Intel Xeon E5504 2.004 MB/800 QC CPU-1 \\
\hline Hewlett Packard & CPU, XEON E5504, CPU 1 & E5504 & Intel Xeon E5504 2.004 MB/800 QC CPU-1 \\
\hline Hewlett Packard & CPU, XEON E5504, CPU 2 & E5504 & Intel Xeon E5504 2.004 MB/800 QC CPU-2 \\
\hline
\end{tabular}




\begin{tabular}{|c|c|c|c|}
\hline Manufacturer & Nomenclature & Part Number & Description \\
\hline Hewlett Packard & CPU, XEON E5504, CPU 2 & E5504 & Intel Xeon E5504 2.004 MB/800 QC CPU-2 \\
\hline Hewlett Packard & CPU, XEON E5530, CPU 1 & DL380G6 & $\begin{array}{l}\text { HP DL380 G6 Intel }{ }^{\circledR} \text { Xeon }^{\circledast} \text { E5530 (2.40GHz/4-core/8MB/80W) FIO } \\
\text { Processor Kit (1st) }\end{array}$ \\
\hline Hewlett Packard & CPU, XEON E5530, CPU 1 & DL380G6 & $\begin{array}{l}\text { HP DL380 G6 Intel }{ }^{\circledR} \text { Xeon }^{\circledR} \text { E5530 (2.40GHz/4-core/8MB/80W) FIO } \\
\text { Processor Kit (1st) }\end{array}$ \\
\hline Hewlett Packard & CPU, XEON E5530, CPU 2 & DL380G6 & $\begin{array}{l}\text { HP DL380 G6 Intel }{ }^{\circledR} \text { Xeon }{ }^{\circledR} \text { E5530 (2.40GHz/4-core/8MB/80W) Processor } \\
\text { Kit (2nd) }\end{array}$ \\
\hline Hewlett Packard & CPU, XEON E5530, CPU 2 & DL380G6 & $\begin{array}{l}\text { HP DL380 G6 Intel }{ }^{\circledast} \text { Xeon }^{\circledast} \text { E5530 (2.40GHz/4-core/8MB/80W) Processor } \\
\text { Kit (2nd) }\end{array}$ \\
\hline Hewlett Packard & ENCLOSURE, MSA, 60 SINGLE & MSA-60 & $\begin{array}{l}\text { HP Storage Works Modular Storage Array (MSA) } 60 \text { single cabinet } \\
\text { enclosure. Not used at all sites. }\end{array}$ \\
\hline Hewlett Packard & HDD CONTROLLER CARD, 4 PORT RAID, SAS SATA & & $3041 E 4$ port SAS/SATA RAID card \\
\hline Hewlett Packard & HDD CONTROLLER CARD, 4 PORT RAID, SAS SATA & $3041 E$ & $3041 E 4$ port SAS/SATA RAID card \\
\hline Hewlett Packard & HDD CONTROLLER CARD, 4 PORT RAID, SAS SATA & $3041 \mathrm{E}$ & $3041 E 4$ port SAS/SATA RAID card \\
\hline Hewlett Packard & $\begin{array}{l}\text { HDD CONTROLLER CARD, SAS } 2 \text { PORT INT AND EXT, } \\
\text { P800 } 512 \text { BBWC }\end{array}$ & DL380G6 & $\begin{array}{l}\text { HP Smart Array P800/512 BBWC 2-ports Int/2-ports Ext PCle x8 SAS } \\
\text { Controller }\end{array}$ \\
\hline Hewlett Packard & $\begin{array}{l}\text { HDD CONTROLLER CARD, SAS } 2 \text { PORT INT AND EXT, } \\
\text { P800 } 512 \text { BBWC }\end{array}$ & DL380G6 & $\begin{array}{l}\text { HP Smart Array P800/512 BBWC 2-ports Int/2-ports Ext PCle x8 SAS } \\
\text { Controller }\end{array}$ \\
\hline Hewlett Packard & HDD, SAS 10K, 146GB & DL380G6 & $\begin{array}{l}\text { HP 146GB 6G SAS 10K rpm SFF (2.5-inch) Dual Port Enterprise 3yr } \\
\text { Warranty Hard Drive }\end{array}$ \\
\hline Hewlett Packard & HDD, SAS 10K, 146GB & DL380G6 & $\begin{array}{l}\text { HP 146GB 6G SAS 10K rpm SFF (2.5-inch) Dual Port Enterprise 3yr } \\
\text { Warranty Hard Drive }\end{array}$ \\
\hline Hewlett Packard & HDD, SAS 15K, 146GB & & HP 146GB SAS 15k (1st) \\
\hline Hewlett Packard & HDD, SAS 15K, 146GB & & HP 146GB SAS 15k (2nd) \\
\hline Hewlett Packard & HDD, SAS 15K, 146GB & & HP 146GB SAS 15k (2nd) \\
\hline Hewlett Packard & HDD, SAS 15K, 146GB & & HP 146GB SAS 15k (1st) \\
\hline Hewlett Packard & HDD, SAS 15K, 146GB & & HP 146GB SAS 15k (2nd) \\
\hline Hewlett Packard & HDD, SAS 15K, 146GB & & HP 146GB SAS 15k (1st) \\
\hline Hewlett Packard & HDD, SAS 7.2K, 1TB & MSA-60 & HP 1TB 7.2k DP MDL SAS 1y Wty HDD \\
\hline Hewlett Packard & KEYBOARD, PS2 & & HP PS/2 Standard Keyboard \\
\hline Hewlett Packard & KEYBOARD, PS2 & & HP PS/2 Standard Keyboard \\
\hline Hewlett Packard & KEYBOARD, PS2 & & HP PS/2 Standard Keyboard \\
\hline Hewlett Packard & KIT, LOCALIZATION & & Z600 Localization Kit \\
\hline
\end{tabular}




\begin{tabular}{|c|c|c|c|}
\hline Manufacturer & Nomenclature & Part Number & Description \\
\hline Hewlett Packard & KIT, LOCALIZATION & & Z600 Localization Kit \\
\hline Hewlett Packard & KIT, LOCALIZATION & & Z600 Localization Kit \\
\hline Hewlett Packard & $\begin{array}{l}\text { KIT, US RACKMOUNT, KEYBOARD MOUSE AND } \\
\text { MONITOR }\end{array}$ & TFT7600 & HP TFT7600 US Rackmount Keyboard, Mouse, 17 inch monitor \\
\hline Hewlett Packard & MEMORY KIT, DDR3-1333, 4GB 4X1 & & HP 4GB (4x1GB) DDR3-1333 ECC 2-CPU RAM \\
\hline Hewlett Packard & MEMORY KIT, DDR3-1333, 4GB 4X1 & & HP 4GB (4x1GB) DDR3-1333 ECC 2-CPU RAM \\
\hline Hewlett Packard & MEMORY KIT, DDR3-1333, 4GB 4X1 & & HP 4GB (4x1GB) DDR3-1333 ECC 2-CPU RAM \\
\hline Hewlett Packard & MEMORY KIT, DDR3-1333, 8GB 1X8 & DL380G6 & $\begin{array}{l}\text { HP 8GB (1x8GB) Dual Rank x4 PC3-10600 (DDR3-1333) Registered CAS-9 } \\
\text { Memory Kit }\end{array}$ \\
\hline Hewlett Packard & MEMORY KIT, DDR3-1333, 8GB 1X8 & DL380G6 & $\begin{array}{l}\text { HP } 8 \text { GB (1x8GB) Dual Rank x4 PC3-10600 (DDR3-1333) Registered CAS-9 } \\
\text { Memory Kit }\end{array}$ \\
\hline Hewlett Packard & MONITOR, FLAT PANEL, 19 INCH & HPL 1910 & HP 19 inch LCD monitor \\
\hline Hewlett Packard & MONITOR, FLAT PANEL, 19 INCH & HPL 1910 & HP 19 inch LCD monitor \\
\hline Hewlett Packard & MONITOR, FLAT PANEL, 20 INCH & LP2065 & HP 20 inch LCD monitor \\
\hline Hewlett Packard & MONITOR, FLAT PANEL, 20 INCH & LP2065 & HP 20 inch LCD monitor \\
\hline Hewlett Packard & MONITOR, FLAT PANEL, 20 INCH & LP2065 & HP 20 inch LCD monitor \\
\hline Hewlett Packard & MOUSE, PS2, OPTICAL & & HP PS/2 Optical Scroll Mouse \\
\hline Hewlett Packard & MOUSE, PS2, OPTICAL & & HP PS/2 Optical Scroll Mouse \\
\hline Hewlett Packard & MOUSE, PS2, OPTICAL & & HP PS/2 Optical Scroll Mouse \\
\hline Hewlett Packard & NETWORK CARD, PCIE, DUAL PORT GIGABIT & DL380G6 & HP NC382T PCI Express Dual Port Multifunction Gigabit Server Adapter \\
\hline Hewlett Packard & NETWORK CARD, PCIE, DUAL PORT GIGABIT & DL380G6 & HP NC382T PCI Express Dual Port Multifunction Gigabit Server Adapter \\
\hline Hewlett Packard & NETWORK CARD, PCIE, GIGABIT & & Broadcom 5761 Gigabit PCle NIC \\
\hline Hewlett Packard & NETWORK CARD, PCIE, GIGABIT & & Broadcom 5761 Gigabit PCle NIC \\
\hline Hewlett Packard & NETWORK CARD, PCIE, GIGABIT & & Broadcom 5761 Gigabit PCle NIC \\
\hline Hewlett Packard & OPTICAL DRIVE, SATA, 16X DVD-ROM & & HP 16x DVD-ROM SATA 1st drive \\
\hline Hewlett Packard & OPTICAL DRIVE, SATA, 16X DVD-ROM & & HP 16x DVD-ROM SATA 1st drive \\
\hline Hewlett Packard & OPTICAL DRIVE, SATA, 16X DVD-ROM & & HP 16x DVD-ROM SATA 1st drive \\
\hline Hewlett Packard & OPTICAL DRIVE, SATA, SLIM DVD-ROM & DL380G6 & HP Slim SATA DVD-ROM Optical Drive \\
\hline Hewlett Packard & OPTICAL DRIVE, SATA, SLIM DVD-ROM & DL380G6 & HP Slim SATA DVD-ROM Optical Drive \\
\hline Hewlett Packard & POWER SUPPLY, AC POWER, 460W & DL380G6 & HP 460W HE Hot Plug AC Power Supply Kit \\
\hline
\end{tabular}




\begin{tabular}{|c|c|c|c|}
\hline Manufacturer & Nomenclature & Part Number & Description \\
\hline Hewlett Packard & POWER SUPPLY, AC POWER, 460W & DL380G6 & HP 460W HE Hot Plug AC Power Supply Kit \\
\hline Hewlett Packard & SOFTWARE, DOWNGRADE TO XP 32 BIT & & Microsoft Vista 32 bit downgrade to XP 32 bit \\
\hline Hewlett Packard & SOFTWARE, DOWNGRADE TO XP 32 BIT & & Microsoft Vista 32 bit downgrade to XP 32 bit \\
\hline Hewlett Packard & SOFTWARE, DOWNGRADE TO XP 32 BIT & & Microsoft Vista 32 bit downgrade to XP 32 bit \\
\hline Hewlett Packard & SPEAKER, SOUND BAR & & HP LCD Speaker Bar \\
\hline Hewlett Packard & SPEAKER, SOUND BAR & & HP LCD Speaker Bar \\
\hline Hewlett Packard & SPEAKER, SOUND BAR & & HP LCD Speaker Bar \\
\hline Hewlett Packard & SWITCH, CAT5 KVM, 0X1X8 & & HP CAT5 0x1×8 KVM Console Switch \\
\hline Hewlett Packard & TAPE DRIVE, MSL & MSL 2024 & HP Storage Workds MSL 2024 Tape Backup Drive. Not used at all sites. \\
\hline Hewlett Packard & VIDEO CARD, NVIDIA QUADRO, 256MB & NVS295 & NVIDIA Quadro NVS295 256 MB \\
\hline Hewlett Packard & VIDEO CARD, NVIDIA QUADRO, 256MB & NVS295 & NVIDIA Quadro NVS295 256 MB \\
\hline Hewlett Packard & VIDEO CARD, NVIDIA QUADRO, 256MB & NVS295 & NVIDIA Quadro NVS295 256 MB \\
\hline Hewlett Packard & WORK STATION & & HP Z600 Workstation Base Unit \\
\hline Hewlett Packard & WORK STATION & & HP Z600 Workstation Base Unit \\
\hline Hewlett Packard & WORK STATION & & HP Z600 Workstation Base Unit \\
\hline Serco Wireless Network & Network & & Communications \\
\hline *** Unknown & FIBER PONE & 60-000381-02 & FIBER POE \\
\hline Advantech & SWITCH, ETHERNET, HARDENED & EKI-7758F & HARDENED ETHERNET SWITCH \\
\hline APC & UPS, 1400VA & SU1400RMXLB3U & 1400VA SMART UPS XL停 \\
\hline Cisco & LIGHTNING ARRESTER & 60-000381-01 & POWER INJECTOR LIGHTNING ARRESTER \\
\hline Cisco & SWITCH, CATALYST & WS-C2955T-12 & CATALYST 2955 SWITCH \\
\hline Cisco & SWITCH, CATALYST & WS-C3750G-24T-E & 3750 CATALYST SWITCH \\
\hline Cisco & SWITCH, CATALYST & WS-C3750G-12S-E & 3750 CATALYST SWITCH \\
\hline Dragonwave & ANTENNA, 26GHZ, 0.3M & VHLP1-26-DW1 & 0.3 METER 26 GHZ ANTENNA \\
\hline Dragonwave & ANTENNA, 28GHZ, 0.3M & VHLP1-28-DW1 & 0.3 METER 28 GHZ ANTENNA \\
\hline Dragonwave & ANTENNA, 28GHZ, 0.6M & VHLP2-28-DW1 & 0.6 METER 28 GHZ ANTENNA \\
\hline Dragonwave & RADIO, COMPACT, 24GHZ & CUSP24UFF & 24 GHZ HORIZON COMPACT RADIO \\
\hline Dragonwave & RADIO, COMPACT, 24GHZ & CUSP24UFC & 24 GHZ HORIZON COMPACT RADIO \\
\hline Dragonwave & RADIO, COMPACT, 28GHZ & CLHP28B2F & 28 GHZ HORIZON COMPACT RADIO \\
\hline
\end{tabular}




\begin{tabular}{|c|c|c|c|}
\hline Manufacturer & Nomenclature & Part Number & Description \\
\hline Dragonwave & RADIO, COMPACT, 28GHZ & CLHP28B2C & 28 GHZ HORIZON COMPACT RADIO \\
\hline Dragonwave & RADIO, COMPACT, 28GHZ & CLHP28B1F & 28 GHZ HORIZON COMPACT RADIO \\
\hline Dragonwave & RADIO, COMPACT, 28GHZ & CLHP28B1C & 28 GHZ HORIZON COMPACT RADIO \\
\hline Dragonwave & RADIO, COMPACT, 28GHZ & CHHP28B2C & 28 GHZ HORIZON COMPACT RADIO \\
\hline Dragonwave & RADIO, COMPACT, 28GHZ & CHHP28B1C & 28 GHZ HORIZON COMPACT RADIO \\
\hline Dragonwave & TEST BENCH, 28GHZ & $60-000327-08-01$ & 28 GHZ TEST BENCH \\
\hline GarrettCom & SWITCH, MEDIA CONVERTER & CSG14H-SX-HD & MEDIA CONVERTER SWITCH \\
\hline Hewlett Packard & KIT, 6 FAN RACK TRAY, 220V & 257414-B21 & 6 Fan Rack Tray Kit 220V \\
\hline ISC Sales & AIR CONDITIONER & NE03012604BX0 & 120V AIR CONDITIONER \\
\hline MW & CONVERTER, 48 VDC & $C D(S D)-25 C-24$ & 48VDC - 24VDC CONVERTER \\
\hline MW & POWER SUPPLY, 48 V AC & RB17137153 & SDR-480P-48 \\
\hline Rittal & CABINET, TS NEMA 4 & TS 8886640 & TS NEMA 4 CABINET \\
\hline Rittal & CABINET, TS NEMA 4 & TS 8886641 & TS NEMA 4 CABINET \\
\hline Rittal & SHELF & 7185035 & Cabinet Shelf \\
\hline SOLA & UPS, DIN RAIL MOUNTABLE & SDU 850 & DIN RAIL MOUNTABLE UPS \\
\hline SORENSEN & POWER SUPPLY, 48 VDC & XFR60-20M13A & 48VDC POWER SUPPLY \\
\hline TDK Lambda & POWER SUPPLY, 48 VDC & DPP480-48-1 & 48VDC POWER SUPPLY \\
\hline Siemens OCR & OCR & & Identification \\
\hline AEG & CARD, UPS SNMP & & AEGUPS SNMP Card \\
\hline AEG & UPS, 3000VA, RACK & & AEG UPS Protect 3000VA Rack \\
\hline Lumenera & $\begin{array}{l}\text { CAMERA, NETWORK, COLOR 1.4MP MEGA } \\
\text { RESOLUTION }\end{array}$ & & Lumenera 1.4 Megapixel Color Network Camera Mega Resolution Camera \\
\hline Pentax & LENS, CAMERA, OBJECTIVE 1.6MM & C1614A & Pentax Camera Objective 1.6mm C1614A (KP) \\
\hline Pentax & LENS, CAMERA, OBJECTIVE 25MM & B2514D & Pentax Camera Objective 25mm B2514D (KP) \\
\hline Pentax & LENS, CAMERA, OBJECTIVE 4.8MM & C418DX & Pentax Camera Objective 4.8mm C418DX (KP) \\
\hline Raytec & ILLUMINATOR, LED, 50 & & RayTech RayLUX 50 LED Illuminator \\
\hline Sick & SENSOR, OPTICAL PAIR, PAIR SEND AND RECEIVE & & Sick Optical Sensor Pair (send/receive) \\
\hline Siemens & CIRCUIT BREAKER, 6KA1 N POL, C.5 & & Circuit Breaker 6KA1+N-pol C0.5 \\
\hline Siemens & CIRCUIT BREAKER, 6KA1 N POL, C1 & & Circuit Breaker 6KA1+N-pol C1 \\
\hline
\end{tabular}




\begin{tabular}{|c|c|c|c|}
\hline Manufacturer & Nomenclature & Part Number & Description \\
\hline Siemens & CIRCUIT BREAKER, 6KA1 N POL, C10 & & Circuit Breaker 6KA1+N-pol C10 \\
\hline Siemens & CIRCUIT BREAKER, 6KA1 N POL, C3 & & Circuit Breaker 6KA1+N-pol C3 \\
\hline Siemens & CIRCUIT BREAKER, 6KA1 N POL, C6 & & Circuit Breaker 6KA1+N-pol C6 \\
\hline Siemens & VOLTAGE PROTECTOR, 230V & & Overvoltage Protection 230V \\
\hline Siemens & VOLTAGE PROTECTOR, CAT6 & & Overvoltage Protection CAT6 \\
\hline Simatic & RACK, PC & & Simatic Rack PC 647B \\
\hline Wago & CONTROLLER, IO ETHERNET & $750-841$ & Wago I/O Ethernet Controller 750-841 \\
\hline Wago & DIGITAL INPUT, IO ETHERNET & 750-504 & Wago I/O Ethernet Digital Input 750-504 \\
\hline Wago & DIGITAL INPUT, IO ETHERNET & $750-432$ & Wago I/O Ethernet Digital Input 750-432 \\
\hline Wago & END MODULE, IO ETHERNET & $750-600$ & Wago I/O Ethernet End Module 750-600 \\
\hline Wago & POWER SUPPLY, IO ETHERNET, IO & $750-612$ & Wago I/O Ethernet Power Supply 750-612 \\
\hline Wago & POWER SUPPLY, IO ETHERNET, IO & 787-612 & Wago I/O Ethernet Power Supply 787-612 \\
\hline Wago & SUPPLY MODULE, IO ETHERNET & $750-610$ & Wago I/O Ethernet Supply Module 750-610 \\
\hline Siemens Wireless Network & Network & & Communications \\
\hline$A D C$ & ACCESSORY, CABLE & ADCCMVIB-3CB20-4 & ADCCMVIB-3CB20-4; ADC; Vertical Cable Management \\
\hline Advantech & CONVERTER, IP & ADAM-6060-BE & ADAM-6060-BE ADTANTECH, ADAM 6020 IP CONVERTER \\
\hline Advantech & POWER SUPPLY, CONTROLLER & PWR-242 & PWR-242-AE ADVANTECH, ADAM 6060 CONTROLLER POWER SUPPLY \\
\hline AGSI & CABLE, DUPLEX, LC SC 2M & $810-147-006$ & 810-147-006 AGSI, FO PATCH CORD 2M LC-SC SMDUPLEX ULTRA \\
\hline APC & BATTERY PACK, 192V, RM & SURT192MXLBP & SURT192MXLBP; APC; 2 APC SMART-UPS, RT 192V RM BATTERY PACK \\
\hline APC & BATTERY PACK, 24V, RS XS & BR24BP & BR24BP APC, BACK-UPS RS/XS 24V BATTERY PACK \\
\hline APC & CABLE, POWER, 16A 230V & AP9876 & AP9876 APC, PWR CORD, 16A, 230V, C19 TO IEC 309 \\
\hline APC & CABLE, POWER, AC & AP9870 & AP9870 APC, AC Power Cables for Equip (IEC 320 C13 and C14) \\
\hline APC & UPS, 1400VA & SUI400RMXLIB3U & SUI400RMXLIB3U; APC; SMART-UPS XL, 1400VA RM 3U 230V \\
\hline APC & UPS, 1500VA & BR15001 & BR15001; APC; BACK-UP UPS, 1500V 230VAC \\
\hline APC & UPS, 6000VA & SURT6000XLI & SURT6000XLI APC, SMARTUPS RT 6000VA XLT OL 208V BLK \\
\hline Bosch & ILLUMINATOR, IR, 850NM & EX26-LED-850M & EX26-LED 850M; EXTREME; EXTREME IR ILLUMINATOR, 850NM, MEDIUM \\
\hline Cisco & FIREWALL & ASA5505-50-BUN-K8 & ASA5505-50-BUN-K8 CISCO, ASA 5505 FIREWALL EDITION BUNDLE \\
\hline Cisco & KIT, VOIP PHONE AND LICENSE & CP-7941G-A-CCME & CP-7941G-A-CCME CISCO, VOIP Phone 7941G W/ONE User license \\
\hline Cisco & SOFTWARE, SECURITY & 2821-HSEC/K9 & CISCO2821-HSEC/K9, ENHANCED SECURITY BUNDLE \\
\hline
\end{tabular}




\begin{tabular}{|c|c|c|c|c|}
\hline & Manufacturer & Nomenclature & Part Number & Description \\
\hline & Cisco & SWITCH, 1000 BASE T & GLC-T & GLC-T CISCO, 1000BASE-T SFP \\
\hline & Cisco & SWITCH, CATALYST & WS-C3750G-24PS-E & WS-C3750G-24PS-E; Cisco; Catalyst 3750G-24PS-E Port POE Switch \\
\hline & Cisco & SWITCH, CATALYST & WS-C3750-24FS-E & WS-C3750-24FS-E; Cisco; Catalyst 3750-24FS Port Switch 100MB \\
\hline & Cisco & TRANCEIVER, LC CONNECTOR SX & GLC-SX-MM & GLC-SX-MM; Cisco; GE SPF, LC Connector SX Tranciever \\
\hline & FIS & CABLE, DUPLEX MALE TO MALE, $1 \mathrm{M}$ & R2MJYM1FISP & R2MJYM1FISP; FIS; DUPLEX, MM, 62.5 MICRON, MTRJSC, 1 METER \\
\hline & FIS & CABLE, DUPLEX, LC SC 2M & D3LYS2FISC & D3LYS2FISC; FIS; DUPLEX, SM, LC-SC, 2 METERS,PATCH CORD \\
\hline & Generic Manufacturer & CABLE, MOLEX, CAT5E & PCD00177BK & PCD00177BK; MOLEX; CAT-5E PATCH CABLE, BLACK, 10 FT \\
\hline & Generic Manufacturer & CABLE, MOLEX, CAT5E & PCD00174BK & PCD00174BK; MOLEX; CAT-5E PATCH CABLE, BLACK, 3 FT \\
\hline & Leviton & CABLE, CAT6 PLUS, 7 FT & $6246007 \mathrm{~L}$ & 6246007L; LEVITON; EXTREME 6+ PATCH CABLE,BLUE,7 FT \\
\hline & Pelco & BRACKET, CAMERA, POLE MOUNT & EM2000 & EM2000; PELCO; CAMERA POLE MOUNT BRACKET \\
\hline & Pelco & HOUSING, CAMERA, OUTDOOR & EH4718-3 & EH4718-3; PELCO; OUTDOOR CAMERA HOUSING, 23OVAC \\
\hline & Pelco & SUN SHIELD, CAMERA & SS4718 & SS4718; PELCO; OUTDOOR HOUSING SUNSHIELD \\
\hline & Prolink & CABLE, CAT6, 2.13M & R5032XGN & R5032XGN; PROLINKL UTP CAT-6 RJ45 CROSS-OVER, GREEN 2.13M \\
\hline & Scaleance & RADIO, WIRELESS & $0 A B 60$ & Scaleance W786 (OAB60) Pro Wireless Radio \\
\hline & Scaleance & RADIO, WIRELESS & OAA60 & Scaleance W786 (OAA60) Pro Wireless Radio \\
\hline & Scaleance & SWITCH, SCALEANCE & $\mathrm{X} 101-1$ & Switch Scaleance X101-1 \\
\hline & Scaleance & SWITCH, SCALEANCE & X310 FE & Switch Scaleance X310 FE \\
\hline & Scaleance & SWITCH, SCALEANCE & X414 3E & Switch Scaleance X414 3E \\
\hline & Scaleance & SWITCH, SCALEANCE & $x 408-2$ & Switch Scaleance X408-2 \\
\hline & Scaleance & SWITCH, SCALEANCE & X308 2LD & Switch Scaleance X308 2LD \\
\hline & Siemens & ANTENNA TERMINATOR, 50 OHM & TI 795-1R & Impedance TI 795-1R \\
\hline & Siemens & CONFIGURATION PLUG & 6GK1900-0АВ00 & C-plug \\
\hline & Siemens & EXTENDER MODULE & EM495-8 & Extender Module EM495-8 \\
\hline & Siemens & MEDIA MODULE & MM492-2LD & Media Module MM492-2LD \\
\hline & Siemens & MEDIA MODULE & MM491-2 & Media Module MM491-2 \\
\hline & Siemens & POWER SUPPLY, 2AC & PS791 & Power Supply PS791 2AC \\
\hline & Sitop & POWER SUPPLY, 2.5A 24V & 6ЕP1333-2BA01 & Sitop Smart 24V/2,5A \\
\hline & Sony & CAMERA, DIGITAL, SURVEILLANCE & SNC-Z2ON & SNC-Z2ON; SONY; DITIGAL SURVEILLANCE CAMERA \\
\hline SLDP & & Fixed & & Detection \\
\hline
\end{tabular}




\begin{tabular}{|c|c|c|c|c|}
\hline & Manufacturer & Nomenclature & Part Number & Description \\
\hline & Sud Chemie & DESICCANT & 0095 & Desiccant \#6 \\
\hline & TSA & ASSEMBLY, MEDIA CONVERTER & 8590 & Media Converter Assembly \\
\hline & TSA & BATTERY, $18 A H$ 12V & $6456 \mathrm{~A}$ & PS-12180-NB 12V 18A/H Battery \\
\hline & TSA & BATTERY, 26AH $12 \mathrm{~V}$ & $6456 \mathrm{~B}$ & BATTERY 12 V 26AMP-HOURS \\
\hline & TSA & BOARD, 8403 & 8403AP & HHV-448D BOARD \\
\hline & TSA & BOARD, 8453 & $8453 G$ & LD-260B \\
\hline & TSA & BOARD, SC-771 & $8751 \mathrm{AA}$ & SC-771 R7 BOARD \\
\hline & TSA & BOARD, SCA-774 & 8754 & SCA-774 BOARD \\
\hline & TSA & CABLE, COAX & 0352 & PM/GN COAX LOT \\
\hline & TSA & CHARGER, 4A 15V & $6463 B$ & CHARGER 15 V 4 AMP \\
\hline & TSA & CONTROLLER, SC-770 & 9411 & SC-770 Controller \\
\hline & TSA & CONTROLLER, SCA-775, ANALOG & 9413 & SCA-775 Controller (Analog) \\
\hline & TSA & DETECTOR, GAMMA & $8200 A$ & DA630 (GAMMA DETECTOR) \\
\hline & TSA & FILTER, AC LINE & 0418 & AC Line Filter \\
\hline & TSA & FUSE, 5 AMP & $7170 \mathrm{~B}$ & 5 AMP FUSE (LD-260) \\
\hline & TSA & LIGHT, FAULT, AMBER & 7354 & AMBER LIGHT (FAULT) \\
\hline & TSA & PMFX4, POTTED & 8699P & PMFX4 BOX ASSEMBLY (POTTED) \\
\hline & TSA & REMOTE CONTROL & $6692 R$ & Remote control \\
\hline & TSA & SENSOR, OCCUPANCY & $6692 W$ & WIZARD OUTDOOR OCCUPANCY SENSOR \\
\hline & TSA & SONALERT & 6440 & SONALERT \\
\hline & TSA & SWITCH, TAMPER, MAGNETIC & 7818 & MAGNETIC TAMPER SWITCH \\
\hline & TSA & VD580, POTTED & 8870AP & GAMMA DETECTOR CAP VD-580 (POTTED) \\
\hline SLDT & & Fixed & & Detection \\
\hline & Sud Chemie & DESICCANT & 0095 & Desiccant \#6 \\
\hline & TSA & ASSEMBLY, MEDIA CONVERTER & 8590 & Media Converter Assembly \\
\hline & TSA & BATTERY, $18 A H$ H $12 \mathrm{~V}$ & $6456 \mathrm{~A}$ & PS-12180-NB 12V 18A/H Battery \\
\hline & TSA & BATTERY, 26AH $12 \mathrm{~V}$ & $6456 B$ & BATTERY 12 V 26AMP-HOURS \\
\hline & TSA & BOARD, 8403 & 8403AP & HHV-448D BOARD \\
\hline & TSA & BOARD, 8453 & $8453 \mathrm{G}$ & LD-260B \\
\hline
\end{tabular}




\begin{tabular}{|c|c|c|c|c|}
\hline & Manufacturer & Nomenclature & Part Number & Description \\
\hline & TSA & BOARD, SC-771 & $8751 \mathrm{AA}$ & SC-771 R7 BOARD \\
\hline & TSA & BOARD, SCA-774 & 8754 & SCA-774 BOARD \\
\hline & TSA & CABLE, COAX & 0353 & TM-850 COAX LOT \\
\hline & TSA & CHARGER, 5A 15V & $6463 C$ & CHARGER 15V 5 AMP \\
\hline & TSA & CONNECTOR & $2725 \mathrm{~A}$ & RS-232 TO RS-485 \\
\hline & TSA & CONNECTOR, MHV, FEMALE TO FEMALE & 2515 & MHV FEM-FEM T CONN \\
\hline & TSA & CONTROLLER, SC-770 & 9411 & SC-770 Controller \\
\hline & TSA & CONTROLLER, SCA-775, ANALOG & 9413 & SCA-775 Controller (Analog) \\
\hline & TSA & DETECTOR, GAMMA, 12 INCH BY 48 INCH & 8244 & GAMMA DETECTOR (12" X 48") \\
\hline & TSA & DRIVER, SIREN & $7346 B$ & SIREN DRIVER (ELK-100) \\
\hline & TSA & FILTER, AC LINE & 0418 & AC Line Filter \\
\hline & TSA & FUSE, 5 AMP & $7170 \mathrm{~B}$ & 5 AMP FUSE (LD-260) \\
\hline & TSA & KIT, SPEED SENSOR & 6661 & SPEED SENSOR KIT \\
\hline & TSA & LIGHT, FAULT, AMBER & 7354 & AMBER LIGHT (FAULT) \\
\hline & TSA & LIGHT, STROBE, BLUE & $7346 \mathrm{~A}$ & STROBE (BLUE) $12 \mathrm{~V}$ \\
\hline & TSA & LIGHT, STROBE, RED & 7346 & STROBE (RED) $12 \mathrm{~V}$ \\
\hline & TSA & PMFX4, POTTED & 8699P & PMFX4 BOX ASSEMBLY (POTTED) \\
\hline & TSA & SENSOR, RADAR & 6661TEU & Radar sensor \\
\hline & TSA & SENSOR, ULTRASONIC & $6661 B$ & ULTRASONIC SENSOR (QT5OU) \\
\hline & TSA & SIREN & $7346 C$ & SIREN (ELK-1RT) \\
\hline & TSA & SWITCH, TAMPER, MAGNETIC & 7818 & MAGNETIC TAMPER SWITCH \\
\hline & TSA & VD580, POTTED & 8870AP & GAMMA DETECTOR CAP VD-580 (POTTED) \\
\hline SLDV & & Fixed & & Detection \\
\hline & Sud Chemie & DESICCANT & 0095 & Desiccant \#6 \\
\hline & TSA & ASSEMBLY, MEDIA CONVERTER & 8590 & Media Converter Assembly \\
\hline & TSA & BATTERY, $18 A H 12 \mathrm{~V}$ & $6456 \mathrm{~A}$ & PS-12180-NB 12V 18A/H Battery \\
\hline & TSA & BATTERY, 26AH $12 \mathrm{~V}$ & $6456 B$ & BATTERY 12 V 26AMP-HOURS \\
\hline & TSA & BOARD, 8403 & 8403AP & HHV-448D BOARD \\
\hline & TSA & BOARD, 8453 & $8453 G$ & LD-260B \\
\hline
\end{tabular}




\begin{tabular}{|c|c|c|c|}
\hline Manufacturer & Nomenclature & Part Number & Description \\
\hline TSA & BOARD, SC-771 & 8751AA & SC-771 R7 BOARD \\
\hline TSA & BOARD, SCA-774 & 8754 & SCA-774 BOARD \\
\hline TSA & BRACKET & 3450 & DETECTOR BRACKETS \\
\hline TSA & CABLE & 0301C & CAT-5 CABLE, 2' \\
\hline TSA & CABLE & 0301E & CABLE (CAT 5, 4 FOOT) \\
\hline TSA & CABLE & 0360A & SLAVE CABLE LOT \\
\hline TSA & CABLE & 0344D & CABLE (3 CON 18G 76") \\
\hline TSA & CABLE & $2802 B$ & CAT5 X-OVER CABLE 7 FT \\
\hline TSA & CABLE & 0360B & POWER CABLE LOT \\
\hline TSA & CABLE & 0360 & MASTER CABLE LOT \\
\hline TSA & CABLE, COAX & 0350 & VM/GN COAX LOT \\
\hline TSA & CHARGER, 5A 15V & $6463 C$ & CHARGER 15V 5 AMP \\
\hline TSA & CONNECTOR & $2725 \mathrm{~A}$ & RS-232 TO RS-485 \\
\hline TSA & CONTROL PLATE, MASTER & 8774 & MASTER CONTROL PLATE \\
\hline TSA & CONTROL PLATE, SLAVE & $8774 \mathrm{~A}$ & SLAVE CONTROL PLATE \\
\hline TSA & CONTROLLER, SC-770 & 9411 & SC-770 Controller \\
\hline TSA & CONTROLLER, SCA-775, ANALOG & 9413 & SCA-775 Controller (Analog) \\
\hline TSA & DETECTOR, GAMMA & $8200 \mathrm{~A}$ & DA630 (GAMMA DETECTOR) \\
\hline TSA & DRIVER, SIREN & $7346 B$ & SIREN DRIVER (ELK-100) \\
\hline TSA & ENCLOSURE & 6987 & MASTER ENCLOSURE \\
\hline TSA & ENCLOSURE & $6985 \mathrm{~A}$ & SLAVE ENCLOSURE W/BACK PLATE (NEMA 3) \\
\hline TSA & ENCLOSURE & 6987A & SLAVE ENCLOSURE \\
\hline TSA & ENCLOSURE & 6985 & MASTER ENCLOSURE W/BACK PLATE (NEMA 3) \\
\hline TSA & FILTER, AC LINE & 0418 & AC Line Filter \\
\hline TSA & FUSE, 5 AMP & $7170 \mathrm{~B}$ & 5 AMP FUSE (LD-260) \\
\hline TSA & HE3 TUBE & $6720 C$ & He3 TUBE (2" X 36") \\
\hline TSA & HEATER STRIP & 7230 & HEATER STRIP WITH THERMOSTAT \\
\hline TSA & KIT, SPEED SENSOR & 6661 & SPEED SENSOR KIT \\
\hline TSA & LEAD SHIELDS & 4804 & 6X31" LEAD SHIELDS \\
\hline
\end{tabular}




\begin{tabular}{|c|c|c|c|}
\hline Manufacturer & Nomenclature & Part Number & Description \\
\hline TSA & LEAD TROUGH & $4804 \times 1$ & 6" X 30" COLLIMATED LEAD TROUGH \\
\hline TSA & LIGHT, FAULT, AMBER & 7354 & AMBER LIGHT (FAULT) \\
\hline TSA & LIGHT, STROBE, BLUE & $7346 \mathrm{~A}$ & STROBE (BLUE) $12 \mathrm{~V}$ \\
\hline TSA & LIGHT, STROBE, RED & 7346 & STROBE (RED) $12 \mathrm{~V}$ \\
\hline TSA & MACH ALUM PLATE, MASTER & $3204 C$ & MACHINED MASTER ALUM. PLATE \\
\hline TSA & MACH ALUM PLATE, SLAVE & $3204 B$ & MACHINED SLAVE ALUM. PLATE \\
\hline TSA & MOUNT & $3450 \mathrm{D}$ & POWER SUPPLY MOUNT \\
\hline TSA & PIPE CLAMPS & 3470 & PIPE CLAMPS FOR He3 TUBES \\
\hline TSA & PMFX4, POTTED & $8699 P$ & PMFX4 BOX ASSEMBLY (POTTED) \\
\hline TSA & POLY BLOCKS & 4875 & LARGE POLY BLOCKS (2 HOLE) \\
\hline TSA & POWER PLATE & $3204 \mathrm{E}$ & MACHINED ALUMINUM POWER PLATE \\
\hline TSA & POWER PLATE & $8774 B$ & POWER PLATE \\
\hline TSA & SENSOR, ULTRASONIC & $6666 \mathrm{D} 3$ & ULTRASONIC SENSOR (Senix) \\
\hline TSA & SIREN & $7346 C$ & SIREN (ELK-1RT) \\
\hline TSA & SWITCH, TAMPER, MAGNETIC & 7818 & MAGNETIC TAMPER SWITCH \\
\hline TSA & TERMINAL & 2533D & TERMINAL BLOCK 5 (10 POS. W/ GND) \\
\hline TSA & TERMINAL & $2533 C$ & TERMINAL BLOCK 3 (10 POS.) \\
\hline TSA & TERMINAL & $2533 B$ & TERMINAL BLOCK 2 (20 POSITION) \\
\hline TSA & TERMINAL & $2533 \mathrm{~F}$ & AC TERMINAL BLOCK W/5 AMP SWITCH AND SERVICE OUTLET \\
\hline TSA & TERMINAL & $2533 \mathrm{~A}$ & TERMINAL BLOCK 1 (6 POSITION) \\
\hline TSA & TERMINAL & $2533 C$ & TERMINAL BLOCK 3 (10 POS.) \\
\hline TSA & TERMINAL & 2532 & 25 PIN TERMINAL BLOCK, TBX\ \\
\hline TSA & TERMINAL STRIP & $2533 \mathrm{E}$ & Terminal Strip \\
\hline TSA & TIES, CABLE & $3450 \mathrm{C}$ & LARGE TYWRAPS \\
\hline TSA & VD580, POTTED & 8870AP & GAMMA DETECTOR CAP VD-580 (POTTED) \\
\hline ThermoFisher & CAS & & Communications \\
\hline ThermoFisher & ADAPTER & GOVC000493 & MOXA ADAPTOR \\
\hline ThermoFisher & ADAPTER, PROTECTIVE & MEVE339 & PROTECTIVE ADAPTER \\
\hline ThermoFisher & AIR CONDITIONER, XMHC & VEIN93 & AHP-1200 XMHC AIR CONDITIONER \\
\hline
\end{tabular}




\begin{tabular}{|c|c|c|c|}
\hline Manufacturer & Nomenclature & Part Number & Description \\
\hline ThermoFisher & ASSEMBLY, CABLE HARNESS, EXT TX & YP11702230A & ASSY, CABLE HARNESS, EXT. RX \\
\hline ThermoFisher & ASSEMBLY, CABLE HARNESS, EXT TX & YP11702230B & ASSY, CABLE HARNESS, EXT. RX \\
\hline ThermoFisher & ASSEMBLY, CABLE HARNESS, EXT TX & YP11702228A & ASSY, CABLE HARNESS, EXT. TX \\
\hline ThermoFisher & ASSEMBLY, CABLE HARNESS, EXT TX & YP11702228B & ASSY, CABLE HARNESS, EXT. TX \\
\hline ThermoFisher & ASSEMBLY, CABLE, 12 VDC FAN & YP11702479 & CABLE ASSEMBLY, 12VDC FAN \\
\hline ThermoFisher & ASSEMBLY, JUNCTION BOX & GOVA000102 & JUNCTION BOX ASSEMBLY \\
\hline ThermoFisher & ASSEMBLY, LH, RX(B) SENSOR & YP11702251-1 & ASSY LH, RX(B) SENSOR \\
\hline ThermoFisher & ASSEMBLY, LH, TX(A) SENSOR & YP11702251 & ASSY LH, TX(A) SENSOR \\
\hline ThermoFisher & ASSEMBLY, NAI, COMPLETE & YP11702006 & ASSY, NAI COMPLETE \\
\hline ThermoFisher & ASSEMBLY, PCB, LED CIRCUIT & $5687 C$ & LED CIRCUIT PCB ASSY \\
\hline ThermoFisher & ASSEMBLY, RH, RX(A) SENSOR & YP11702250-1 & ASSY RH, RX(A) SENSOR \\
\hline ThermoFisher & ASSEMBLY, RH, TX(B) SENSOR & YP11702250 & ASSY RH, TX(B) SENSOR \\
\hline ThermoFisher & ASSEMBLY, SURGE ARRESTOR, 40A 120V & MEVE367 & 120V 40A SURGE ARRESTOR ASSEMBLY \\
\hline ThermoFisher & BOARD, CONTROLLER, BATTERY & $5681 \mathrm{~A}$ & BATTERY CONTROLLER BOARD \\
\hline ThermoFisher & BOARD, COVERSION, 24V DC DIGITAL IO & CONV1 & Conversion Board, 24 VDC Digital I/O \\
\hline ThermoFisher & BOARD, DISPLAY & GOVC000167 & DISPLAY BOARD \\
\hline ThermoFisher & BOARD, MAIN CONTROL & GOVC000172 & MAIN CONTROL BOARD \\
\hline ThermoFisher & CABLE, CL, BFR/SPL TO SURGE PROTECTOR & YP11702499 & CBL, CL BFR/SPL PCB TO SURG PR \\
\hline ThermoFisher & CABLE, DC POWER, BOTTOM FAN & YP11702488 & CABLE, DC POWER TO BOTTOM FAN \\
\hline ThermoFisher & CABLE, DC POWER, TOP FAN & YP11702487 & CABLE, DC POWER TO TOP FAN \\
\hline ThermoFisher & CABLE, FIBER, 30 FT SC & YP11702523 & 30 FT. SC FIBER CABLE \\
\hline ThermoFisher & CABLE, FIBER, CONV TO COUPLER & YP11702500 & FIBER CABLE, CONV TO COUPLER \\
\hline ThermoFisher & CABLE, LAN, POE SW RS485 LAN CON & YP11702497 & CBL, LAN, POE SW-RS485/LAN CON \\
\hline ThermoFisher & CABLE, LAN, POE SW TO TEMP BS & YP11702496 & CABLE, LAN, POE SW TO TEMP BS \\
\hline ThermoFisher & CABLE, POWER, 48 VDC TO MON PCB & YP11702492 & CABLE, 48VDC TO PWR MON PCB \\
\hline ThermoFisher & CABLE, POWER, IN TO TB & YP11702489 & CABLE, POWER IN TO POWER TB \\
\hline ThermoFisher & CABLE, POWER, MON PCB TO 48 VDC TB & YP11702494 & CABLE, PWR MON PCB TO 48VDC TB \\
\hline ThermoFisher & CABLE, POWER, TB TO 48 VDC PS & YP11702490 & CABLE, PWR TB TO 48VDC PS \\
\hline ThermoFisher & CHARGER, BATTERY, 24V DC & GOVC000166 & CHARGER BATTERY 24VDC \\
\hline
\end{tabular}




\begin{tabular}{|c|c|c|c|}
\hline Manufacturer & Nomenclature & Part Number & Description \\
\hline ThermoFisher & CIRCUIT BREAKER, 1P 120 VAC, 10A & FUCB24 & Circuit Breaker, 10A, 1P, 120 VAC \\
\hline ThermoFisher & CIRCUIT BREAKER, 1P 120 VAC, 15A & FUCB25 & Circuit Breaker, 15A, 1P, 120 VAC \\
\hline ThermoFisher & CIRCUIT BREAKER, 1P 120 VAC, 20A & FUCB26 & Circuit Breaker, 20A, 1P, 120 VAC \\
\hline ThermoFisher & CIRCUIT BREAKER, 1P 120 VAC, 2A & FUCB22 & Circuit Breaker, 2A, 1P, 120 VAC \\
\hline ThermoFisher & CIRCUIT BREAKER, 1P 120 VAC, 5A & FUCB23 & Circuit Breaker, 5A, 1P, 120 VAC \\
\hline ThermoFisher & COMPACT FLASH, 8 GB & GOVC000119 & INDUSTRIAL COMPACT FLASH 8 GB \\
\hline ThermoFisher & COUPLER, SC DUPLEX, MALE TO MALE & COHD282 & COUPLING, 3M SC DUPLEX MM \\
\hline ThermoFisher & CRATE & & Crate \\
\hline ThermoFisher & FAN, CIRCULATION, 115 VAC 4 INCH AXIAL & FNAC10 & Circulation Fan, 115 VAC, 4" Axial \\
\hline ThermoFisher & FILTER, SINGLE PHASE, EMI/RFI & LFTR001 & SINGLE PHASE EMI/RFI FILTER \\
\hline ThermoFisher & FUSE, 10A & GOVC000295 & 10A Fuse \\
\hline ThermoFisher & FUSE, 8A & GOVC000296 & $8 \mathrm{~A}$ Fuse \\
\hline ThermoFisher & HARNESS, WIRE, COMM POWER & ER6087C & WIRE HARNESS COMM/POWER \\
\hline ThermoFisher & HARNESS, WIRE, DC POWER & YP11702481 & WIRE HARNESS FOR DC POWER \\
\hline ThermoFisher & HARNESS, WIRE, NAI DET EXT 1 & YP11702483 & WIRE HARNESS, NAI DET, EXT. \#1 \\
\hline ThermoFisher & HARNESS, WIRE, NAI DET EXT 2 & YP11702484 & WIRE HARNESS, NAI DET, EXT. \#2 \\
\hline ThermoFisher & HARNESS, WIRE, NAI DET EXT 3 & YP11702485 & WIRE HARNESS, NAI DET, EXT. \#3 \\
\hline ThermoFisher & HARNESS, WIRE, NAI DET EXT 4 & YP11702486 & WIRE HARNESS, NAI DET, EXT. \#4 \\
\hline ThermoFisher & HUB, TEMPERATURE, E4 & MEVE325 & TEMPERATURE HUB E-4 \\
\hline ThermoFisher & IO UNIT, ETHERNET & MEVE361 & Ethernet I/O Unit, EtherTrak \\
\hline ThermoFisher & KIT, HE3 & GOVA000219 & He3 Kit \\
\hline ThermoFisher & KIT, PCS & GOVA000218 & PCS Kit \\
\hline ThermoFisher & $\mathrm{KIT}, \mathrm{RSP}$ & GOVA000216 & RSP Kit \\
\hline ThermoFisher & KIT, UPS & GOVA000217 & UPS Kit \\
\hline ThermoFisher & KIT, VIS & GOVA000220 & Vis Kit \\
\hline ThermoFisher & LATCH, 3 POINT WITH KEY, ALTERED & ZP11702077 & LATCH, 3-POINT, W/KEY, ALTERED \\
\hline ThermoFisher & MODULE, $4 X 4$ & MEVE356 & Module Insert, 4 X 4 \\
\hline ThermoFisher & MODULE, FIBER INSERT, SFF & MEVE355 & Fiber Module Insert, SFF \\
\hline ThermoFisher & MODULE, LANTRONIX & GOVC000171 & LANTRONIX MODULE \\
\hline
\end{tabular}




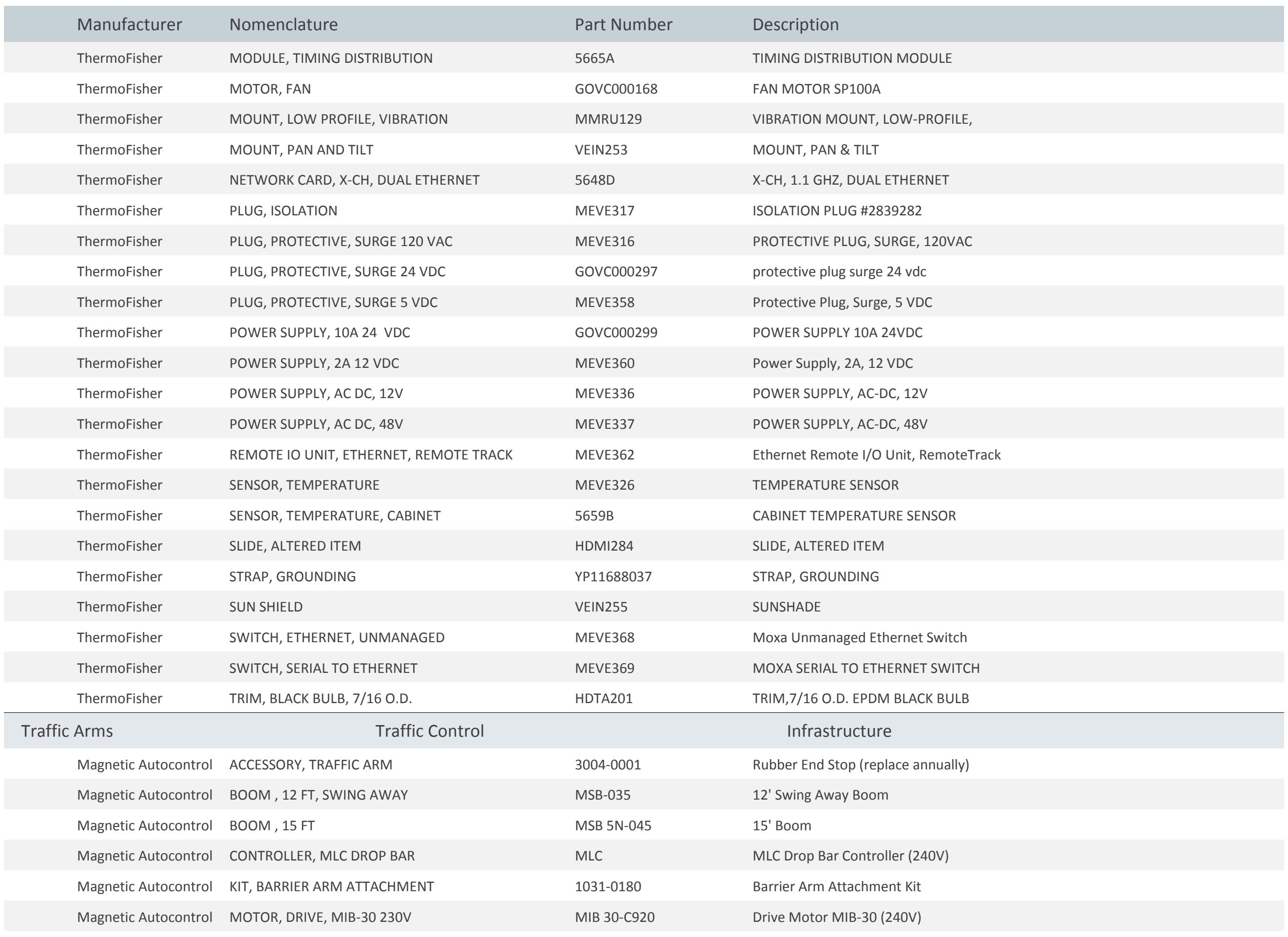




\begin{tabular}{|c|c|c|c|c|}
\hline & Manufacturer & Nomenclature & Part Number & Description \\
\hline & Magnetic Autocontrol & MOTOR, DRIVE, MIB-40 240V & MIB 40-C920 & Drive Motor MIB-40 (240V) \\
\hline & Magnetic Autocontrol & SET, SWING AWAY FLANGE & 1031-5052 & Swing Away Flange-Set \\
\hline & Magnetic Autocontrol & WIRE, INDUCTION LOOP & 90000.0001 & Induction Loop Wire \\
\hline Utility & Panels & Power & & Infrastructure \\
\hline & $A B B$ & CIRCUIT BREAKER, 2P, 40A & F2020AC-40/0.03 & 2-pole, 40amp, 100ma trip \\
\hline & Allen Bradley & CIRCUIT BREAKER, 1P 230V AC, 20 & S201U-K20 & 1-Circuit Breaker 20 AMP, 230V AC, 10KA Rating \\
\hline & Allen Bradley & CIRCUIT BREAKER, 1P 230V AC, 40A & S201U-K40 & 1-Pole Circuit Breaker $40 \mathrm{Amp}, 230 \mathrm{~V} \mathrm{AC}$ \\
\hline & Allen Bradley & CIRCUIT BREAKER, 1P 230V AC, 50A & S201U-K50 & 1-Pole Circuit Breaker $50 \mathrm{Amp}, 230 \mathrm{~V} \mathrm{AC}$ \\
\hline & Allen Bradley & CIRCUIT BREAKER, 1P 230V AC, 5A & S201U-K50 & 1-Pole Circuit Breaker 5 AMP, $230 \mathrm{~V}$ AC \\
\hline & Allen Bradley & CIRCUIT BREAKER, 1P DIN RAIL MOUNT, 6A & 1492-SP1C060 & 1 pole 6 amp, DIN rail mount breaker \\
\hline & Allen Bradley & CIRCUIT BREAKER, 1P PLUS NEUTRAL, 20A & 1492-SP1B200-N & 1 pole plus neutral $20 \mathrm{amp}$, DIN rail mount breaker \\
\hline & Allen Bradley & CIRCUIT BREAKER, 1P, 10A & 1492-SP1C100 & 1-Pole 10 amp Circuit Breaker \\
\hline & Allen Bradley & CIRCUIT BREAKER, 2P, 50A & 1492-SP2C500 & 2-Pole 50 AMP Circuit Breaker \\
\hline & Allen Bradley & CIRCUIT BREAKER, $5 \mathrm{~A}$ & 1492-SP1C050 & 5 Amp circuit breaker \\
\hline & Allen Bradley & MODULE, TIMING & 700-HT3 & Timing Module \\
\hline & Allen Bradley & RELAY, TUBE BASE, WITH PILOT LIGHT & 700-HA33Z12-4 & Tube Base Relay w/pilot light \\
\hline & Automation Direct & CABLE, PC/PLC PROGRAM, 9PIN SERIAL TO RJ45 & D2-DSCBL & PC/PLC Prog. Cable, 9-PIN Serial to RJ-45 \\
\hline & Automation Direct & CONTROLLER, PROGRAMMABLE LOGIC & D0-060DR-D & Programmable Logic Controller \\
\hline & Automation Direct & KIT, CABLE, PLC PROGRAMMING & DA-DSCBL & PLC Programming Cable Kit \\
\hline & Automation Direct & MODULE, COMMUNICATION, ETHERNET & HO-ЕСОМ100 & ETHERNET COMMUNICATION MODULE \\
\hline & Automation Direct & PROGRAMMABLE LOGIC CONTROLLER HAND HELD & D2-HPP & PLC Hand Held \\
\hline & Automation Direct & SOFTWARE, PLC PROGRAMMING & PC-DSOFT5 & PLC Programming software \\
\hline & Belkin & CABLE, CAT5E & UTP 4 pairs Cat5e & Cat5e Patch Cable \\
\hline & B-Line & HEATER, CONVECTION, 30W & $\mathrm{EH}-30$ & 30 watt B-Line/Cooper Convection heater \\
\hline & B-Line & HEATER, CONVECTION, 60W & CAW EH-60 & 60 watt convection heater, 120 - 230vac \\
\hline & Bussman & FUSE, 12 V DC 5A & AGC-5 & $12 \mathrm{~V} D C, 5 \mathrm{~A}$ Fuses \\
\hline & Bussman & FUSE, 15A & LP-CC-15 & 15A Fuses \\
\hline & Cables Plus & PANEL INSERT, PATCH, FIBER & AP-VCC1CB & Fiber Patch Panel Insert \\
\hline & Corcom & VOLTAGE REGULATOR & 3VDK1 [or 6VN1] & Corcom Voltage Regulator \\
\hline
\end{tabular}




\begin{tabular}{|c|c|c|c|}
\hline Manufacturer & Nomenclature & Part Number & Description \\
\hline Finder & RELAY BASE & 90.03 & Relay Base \\
\hline GarrettCom & MEDIA CONVERTER & CS14-1SC-i & Multimode Media Converter (indoor only) \\
\hline GarrettCom & SWITCH, CONVERTER, MULTIMODE & CS14H-SC-12VDC & Converter/Switch (Multi Mode) \\
\hline GarrettCom & SWITCH, CONVERTER, SINGLE MODE & CS14H-SSC-12VDC & Converter/Switch (Single Mode) \\
\hline GarrettCom & SWITCH, ETHERNET & $\mathrm{ES} 42 \mathrm{H}-2 \mathrm{SSC}-\mathrm{Hi}$ & Ethernet Switch (Magnum Singlemode) \\
\hline GarrettCom & SWITCH, MEDIA CONVERTER & CS14P-SC-12VDX & Media Converter Switch (Magnum) \\
\hline Generic Manufacturer & FUSE, $1.5 \mathrm{~A}$ & GGC-1/2 & 1.5amp fuse \\
\hline Generic Manufacturer & FUSE, 15A, CLASS CC & KTK-CC-15 & Fuse, 15 amp, Class CC \\
\hline Generic Manufacturer & FUSE, 5A & AGC-4 & Fuse, 5amp \\
\hline Generic Manufacturer & FUSE, 7A & GGC-7 & 7amp fuse \\
\hline Genesis & HYGROSTAT, 120-230V, ETF012 & G01203.9-00 & Hygrostat, ETF-012, 120 - 230vac \\
\hline George Fix Industries & SWITCH, TAMPER, MAGNETIC & $50 F-12 W G$ & Magnetic tamper switch, wide gap, $25 \mathrm{~mm}$ \\
\hline Grainger & PADLOCK, KEYED ALIKE & $4 \mathrm{YH} 24$ & Padlock, keyed alike \\
\hline Hoffman & LENS, HOFFMAN FIXTURE & 87576853 & Lens for Hoffman Fixture ALF25M18 \\
\hline Hoffman & SEAL, 2 INCH HOLE & 59760 & 2 inch hole seal \\
\hline $\begin{array}{l}\text { Honeywell } \\
\text { Microswitch }\end{array}$ & LIMIT SWITCH, PIN HEAD WITH ROLLER LEVER, SPDT & $B Z-2 R L 2-A 2$ & Limit switch, pin head w/roller lever, SPDT \\
\hline Leviton & CABLE, FIBER OPTIC, 1M & Cat\#62DSC-M01 & 1 meter Fiber Optic Patch Cord (multimode) \\
\hline Leviton & CABLE, FIBER OPTIC, 3M & Cat\#62DSC-M03 & 3 meter Fiber Optic Patch Cord (multimode) \\
\hline Leviton & POWER SUPPLY & $12 \mathrm{~A}$ & Adaptor/Power Supply \\
\hline Phillips & LAMP & F15T8/CW/ALTO & Lamp for Light Fixture \\
\hline Phoenix Contact & RELAY, AUTOMATION DIRECT & PLC-RSC-2300 & Automation Direct RELAY \\
\hline Phoenix Contact & TERMINAL, FUSIBLE, WITH LED & UT 6-HESI & FUSIBLE TERMINAL WITH LED \\
\hline Weidmuller & BATTERY, BACKUP UNIT & 9916280012 & Battery Backup Unit \\
\hline Weidmuller & POWER SUPPLY, 12 VDC, $160 \mathrm{~W}$ & 9925340012 & 12V DC, 160W Power Supply \\
\hline Zerosurge & SURGE SUPPRESSOR & TVS230LC10 & SURGE SUPPRESSOR \\
\hline
\end{tabular}




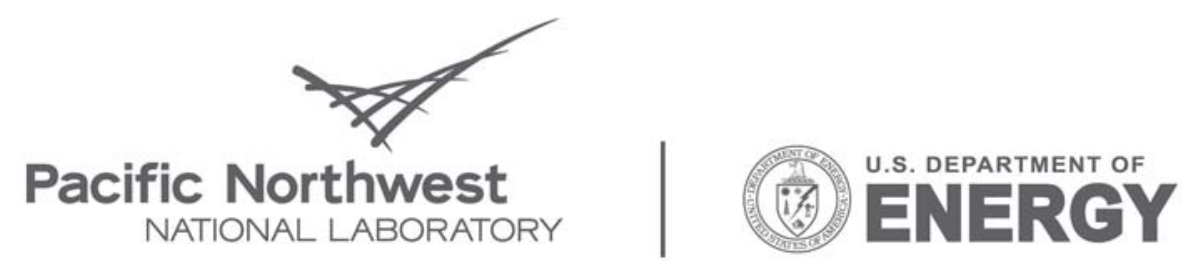

Proudly Operated by Battelle Since 1965

902 Battelle Boulevard

P.O. Box 999

Richland, WA 99352

1-888-375-PNNL (7665)

www.pnl.gov 